\title{
DIVERSIDADE DE MOSQUITOS \\ (DIPTERA: CULICIDAE) EM AMBIENTES DE MATA PRIMÁRIA, MATA RESIDUAL E ÁREA DE CULTIVO IRRIGADO DE ARROZ NO VALE DO RIBEIRA, ESTADO DE SÃO PAULO
}

HELENE MARIKO UENO

Dissertação de Mestrado apresentada ao

Departamento de Epidemiologia

Da Faculdade de Saúde Pública da

Universidade de São Paulo para obtenção do Grau de Mestre.

Área de concentração: Epidemiologia

Orientador: Prof. Cat.

OSWALDO PAULO FORATTINI

São Paulo

2000

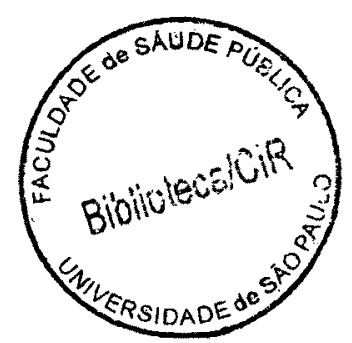


Autorizo, exclusivamente para fins acadêmicos e científicos, a reprodução total ou parcial desta dissertação, por processos fotocopiadores.

Assinatura:

Data: 
Dedico aos meus pais,

Kyo e Siyoko, com amor e gratidão. 


\section{AGRADECIMENTOS}

Ao Prof. Cat. Oswaldo PAUlo ForatTIN pelos ensinamentos, orientação prestada e constante apoio no decorrer de todo o trabalho.

À Profa. Dra. MARIA ANICE MUREB SALLUM pela orientação na definição do tema e elaboração do projeto que resultou neste trabalho.

À CAPES (Coordenação de Aperfeiçoamento de Pessoal de Nível Superior) pelo auxílio financeiro.

Ao Prof. Dr. ARício XAVIER LinHARES e Prof. Dr. EduARDo MASSAD, membros da banca examinadora, pelas sugestões e correções que muito contribuíram com $o$ trabalho.

Ao Prof. Dr. Newton Goulart MadeIRA pelo acompanhamento do trabalho, discussões, críticas e sugestões de leitura, fundamentais à realização deste.

Ao Prof. Dr. Delsio NATAL pela atenção de sempre, tanto nas discussões sobre o trabalho quanto na leitura e correção do texto.

À Profa. Dra. INÁ KAKITANI pelas discussões e esclarecimentos sobre o Projeto Temático "Culicidae do agro-ecossistema irrigado e seu significado epidemiológico" (FAPESP, processo 90/3371-6), cujos dados foram aqui utilizados.

Ao ARISTIDES FERNANDES pelos esclarecimentos a respeito da identificação de espécies.

Ao DANIEL C. FLORES pela concessão dos mapas e ao MARCELLUS WLLIAM JANES e Luís FILIPE MUCCI pela edição dos mesmos. 
Ao Prof. Dr. NELSON BERNARdi e à LIA FIGUEREDo BERNARDI GIMENEZ pela revisão do resumo em inglês.

Ao MARCELO REgos pela generosa contribuição com as cópias do trabalho.

Ao MARCUS VINICIUS FERREIRA pelo auxilio na organização do banco de dados aqui utilizado.

Às companheiras de trabalho KeIlla MIKI KOBAYASH, ROSELI LA CORTE e ZORAIDA DEL CARMEN GRILLO FERNÁNDEZ, pelo constante apoio e sugestões ao trabalho, principalmente no preparo para a defesa desta dissertação.

A todos aqueles que, de uma forma ou outra, contribuíram para a realização deste trabalho. 


\section{RESUMO}

Ueno HM. Diversidade de mosquitos (Diptera: Culicidae) em ambientes de mata primária, mata residual e área de cultivo irrigado de arroz no Vale do Ribeira, Estado de São Paulo. São Paulo, 2000 [Dissertação de Mestrado - Faculdade de Saúde Pública da USP].

Objetivo. Comparar a fauna culicidiana em ambiente de mata primária, mata residual e área de cultivo irrigado de arroz na Região do Vale do Ribeira (SP), mediante análise: 1) de composição faunística, quanto ao número de espécies e indivíduos; 2) diversidade; 3) distribuição das espécies dominantes; 4) constância de espécies; 5) similaridade entre os ambientes e 6) discussão sobre espécies de importância médica. Material e Método. Os dados referem-se às coletas de mosquitos adultos em armadilha tipo Shannon com fonte luminosa e dois coletores com aspirador manual, no período das 17 às $22 \mathrm{~h}$, de janeiro de 1992 a abril de 1995 , referentes ao Projeto Temático "Culicidae do agro-ecossistema irrigado e seu significado epidemiológico" (FAPESP, processo 90/3371-6). Para cada tipo de ambiente foram traçadas curvas cumulativas do número de espécies e indivíduos. $\mathrm{A}$ diversidade de espécies foi calculada através dos índices de Margalef, Margalef Padronizado e $\alpha$ de Fisher. Para a dominância de espécies calculou-se o índice de Berger-Parker. A constância de espécies foi avaliada pelo índice de constância e a similaridade, pelo coeficiente de Sorensen. Resultados. O número de espécies foi maior na mata residual e menor na arrozal, ocorrendo o inverso com o número de individuos. Os índices de diversidade comportaram-se semelhantemente, sendo maiores na mata residual e menores no arrozal. As espécies dominantes foram: Anopheles cruzii na mata primária, Aedes serratus na mata residual e Anopheles albitarsis (espécie B) no arrozal, todas elas de importância médica. Em relação à constância e similaridade, o arrozal destacou-se dos demais ambientes, pois apresentou mais espécies constantes e menos espécies compartilhadas com as matas primária e residual do que estas entre si. Discussão e Conclusões. Para todas as análises realizadas, o número de coletas e a técnica empregada interferiram nos resultados, provavelmente em função de caracteristicas biológicas e comportamentais 
das espécies. Procurou-se integrar fatores que possam justificar maior diversidade em mata residual, tais como presença de características de mata primária em grau variável, contiguidade com áreas abertas e proximidade com o arrozal. Neste tipo de ambiente, a homogeneidade de recursos e a previsibilidade ambiental condicionada pelo ciclo de cultivo do arroz, propiciaram a formação de criadouros que favoreceram algumas espécies. Daí a riqueza e diversidade menores do que nas matas e a maior proporção de espécies constantes e mais abundantes. Quanto ds espécies raras, de um modo geral, podem ser encaradas como em processo de adaptação, uma vez que as áreas estudadas, representando diferentes graus de impacto, apresentaram padrões de diversidade distintos.

Descritores. Culicidae. Diversidade. Cultivo irrigado de arroz. 


\section{SUMMARY}

Ueno HM. Diversidade de mosquitos (Diptera: Culicidae) em ambientes de mata primária, mata residual e área de cultivo irrigado de arroz no Vale do Ribeira, Estado de São Paulo. [Mosquito (Diptera: Culicidae) diversity in a primitive forest, a residual forest and an irrigated rice culture at the Ribeira Valley, São Paulo State] São Paulo (BR), 2000 [Dissertação de Mestrado - Faculdade de Saúde Pública da USP].

Objective. To compare the mosquito fauna in a primitive forest, a residual forest and an irrigated rice culture at the Ribeira Valley (SP), through the analysis of: 1) fauna composition, as the number of species and the number of individuals; 2) species diversity; 3) dominant species distribution; 4) species constancy; 5) similarity between environments, and 6) medical important species. Material and Methods. Adult mosquitoes were collected using Shannon traps with incandescent light, and two collectors using manual aspirator, from 5 P.M. to 10 P.M., from Jan/92 to April/95. Data obtained from the Thematic Project "Culicidae in irrigated agroecossystem and their epidemiological meaning" (FAPESP process 90/3371-6) were used. For each environment, cumulative number of species and individuals were plotted. Species diversity was calculated using Margalef, standardized Margalef and Fisher's $\alpha$ indexes. The Berger-Parker index was calculated to determine species dominance. Species constancy was evaluated by the constancy index, and the similarity by the Sorensen coefficient. Results. The highest species number was found in the residual forest and the lowest in the rice field; the opposite ocurred with the number of individuals. Similarly, the highest diversity indexes were found in the residual forest and the lowest in the rice field. The dominant species were: Anopheles cruzii in the primitive forest, Aedes serratus in the residual forest and Anopheles albitarsis (species B) in the rice field. These three species have medical importance. In relation to constancy and similarity, the rice field differs from other environments because it has more constant species and less species shared with the primitive and the residual forest than these two environments compared to each other. Discussion and Conclusions. In all analyses, number of collections and the thechniques 
employed interfered in the results, probably due to biological and behavioural characteristics of the species. The factors that may justify higher diversity in the residual forest are: presence of primitive forest characteristics, contiguous presence of open areas and proximity to rice fields. In this environment, homogeneity of resources and environment predictability, conditioned by the rice culture cycle, established breeding sites which may have favoured some species. Thus, the smaller richness and diversity than in the forest and the greater proportion of more abundant and constant species. The presence of rare species, may be considered as adaptating process, since the studied areas represent different degrees of human interference and show distinct diversity patterns.

Descriptors: Culicidae. Diversity. Irrigated rice culture. 


\section{ÍNDICE}

Introdução 1

Objetivo 5

Objetivos específicos 5

Características do Vale do Ribeira $\quad 6$

Localidades estudadas $\quad \mathbf{8}$

Material e Método $\quad 12$

$\begin{array}{ll}\text { Resultados } & 15\end{array}$

1. Número de espécies (S) e número de indivíduos $(\mathrm{N}) \quad 15$

1.1. S e N em ambiente de mata primária $\quad 15$

1.2. $\mathrm{S}$ e $\mathrm{N}$ em ambiente de mata residual $\quad 19$

1.3. $\mathrm{S}$ e $\mathrm{N}$ em área de cultivo irrigado de arroz 24

2. Diversidade $\quad 27$

3. Dominância 32

4. Constância 36

5. Similaridade $\quad 39$

6. Espécies de importância médica 39

6.1. Anopheles $\quad 39$

6.2. Aedes $\quad 40$

6.3. Culex 40

6.4. Outras espécies $\quad 41$

Discussão $\quad 42$

Considerações Finais $\quad 56$

Referências Bibliográficas $\quad 58$

Apêndice 


\section{INTRODUÇÃO}

Uma característica diferencial importante do homem é que, enquanto os demais organismos tendem a adaptar-se às mudanças ambientais, o homem altera o ambiente para adaptá-lo às suas necessidades, seja desmatando áreas para cultivo, domesticando e selecionando animais e plantas, construindo edificações, explorando recursos naturais e produzindo resíduos (SCHOFIELD 1990)

Segundo FORATTINI (1992), a agricultura representou o maior e mais duradouro impacto da História da humanidade, uma vez que permitiu a substituição do nomadismo pelo sedentarismo. Com o aumento da ocupação de áreas para cultivo e de recursos para sobrevivência, a população humana passou a crescer.

POVOLNÝ (1971) classifica biocenoses em dois tipos: primária ou natural e secundária ou cultural. Neste grupo distinguem-se as espécies que o homem selecionou, isto é, domesticou desde que se tornou sedentário, as quais se adaptaram às condições por ele criadas, estabelecendo a antropobiocenose. Porém várias outras espécies incorporaram-se a ela espontaneamante, ligando-se diretamente ao homem, aos animais domésticos ou abrigando-se na residência. Estas espécies são ditas sinantrópicas.

KOSTROWICKI (1982) definiu sinantropização como o processo de adaptação de vários sistemas ecológicos a condições estabelecidas por atividades humanas e que se tornaram dominantes num dado tempo e espaço.

O fato é que nos últimos 25 anos a população mundial dobrou de tamanho e estima-se que vai dobrar novamente antes de 2050, o que acelera acentuadamente o fenômeno de antropização do globo. Mais de $90 \%$ desse crescimento populacional tem ocorrido nos países em desenvolvimento, cujas prioridades são o aumento da produção de alimentos, mediante a colonização de novas terras e intensificação de esquemas de cultivo (COOSEMANS e MOUCHET 1990).

Esta antropização do globo induz sinantropização de espécies. Diante disso, surgem preocupações de naturezas distintas: aumento da produção, otimização e distribuição adequada de recursos necessários ao homem (água, alimento, energia); e "adaptação" do homem às espécies sinantrópicas. Sobretudo, conhecer aquelas que 
o prejudicam diretamente. Em outras palavras, compreender o padrões de mudança de diversidade biológica e ambiental.

$\mathrm{O}$ conceito de biodiversidade vem sendo debatido entre vários autores (MAGURRAN 1988, BEGON e cols 1996, GASTON 1996, HAYEK e BUZAS 1997), mas há um consenso em considerar o número de unidades e suas frequências relativas. Entende-se por unidade qualquer nível de organização da biosfera - desde genes até ecossistemas em escala biogeográfica.

Do ponto de vista operacional, o mais comum é considerar o nível de espécie. Define-se como riqueza o número de espécies e equidade como o padrão e distribuição de indivíduos entre as espécies. BALTANÁS (1992) adverte que as comunidades são estabelecidas por critérios arbitrários (espaciais, tróficos, taxonômicos etc.) e que podem limitar suas dimensões reais.

Impactos ambientais, como desmatamento, represamento de água, poluição (ar, água, solo) alteram o padrão de diversidade local em basicamente dois sentidos: redução da riqueza de espécies com aumento do endemismo de algumas, e manutenção da riqueza de espécies com alteração de sua composição (DUDLEY e cols 1992, FUJISAKA e cols 1998, citados por SWENGEL 1998, p.1639). Por outro lado, impactos naturais catastróficos podem promover aumento da diversidade em função eliminação da dominância de poucas espécies, característica de ecossistemas saturados (GRANZOW-DELACERDA e cols 1997).

WALSH e colaboradores (1993) escreveram revisão sobre os efeitos do desmatamento sobre doenças veiculadas por artrópodes. O desmatamento ou atividades associadas a ele produziu novos habitats para Anopheles darlingi, que tem causado epidemias de malária na América do Sul. No sudeste asiático e África Ocidental têm ocorrido processos semelhantes com seus respectivos vetores locais. Certas espécies de flebotomíneos (Lutzomyia intermedia, Lu.. longipalpis, Lu.whitmani), originalmente zoofilicas e silvestres têm-se adaptado ao homem como fonte alternativa de sangue, frequentando o peridomicílio e alterando a distribuição de leishmaniose na América do Sul. Várias arboviroses de ciclo enzoótico têm afetado o homem em função de sua penetração em matas e/ou pela aproximação de reservatórios e adaptação de vetores ao ambiente domiciliar. 
DEFOLIART (1987) salienta alguns dos mecanismos de adaptação de vetores de arbovírus, entre eles, a variação geográfica intraespecífica de comportamento e competência vetorial, transmissão horizontal e vertical de vírus, fatores eco e biológicos dos vetores, como resistência a periodos de seca ou restrição de recursos, longevidade dos adultos, sobrevivência da larva, duração do ciclo gonotrófico, fecundidade etc. Tais mecanismos podem decorrer tanto das alterações do homem no ambiente, quanto das medidas de controle aplicadas aos vetores.

BAXTER (1977) escreveu, em revisão sobre reservatórios e barragens, que qualquer que seja a finalidade - suprimento de água, piscicultura, irrigação, produção de energia etc. - qualquer que seja o tamanho, estarão envolvidas importantes alterações. Isso se justifica devido ao fato dos reservatórios e barragens constituírem ecossitema particular, distinto daquele dos lagos naturais e rios.

A despeito dos beneficios econômicos decorrentes dessas atividades, entre os principais problemas, potenciais ou já registrados, decorrentes da construção de reservatórios e barragens destacam-se: expansão de focos e intensificação da transmissão de doenças, bem como introdução de novas doenças. Nesse contexto, na África, a esquistossomose é a doença mais prevalente e, portanto, de grande importância; na Ásia, malária, dengue, febre hemorrágica, encefalite japonesa B, filariose, doenças causadas por helmintos, esquistossomose, doenças causadas por tremátodos, cólera e disenteria; na América do Sul, malária, leishmaniose e febre amarela. Todas essas doenças são endêmicas (OMS 1983).

No que diz respeito à construção de barragens ou reservatórios de água para fins agrícolas, os esquemas de irrigação merecem destaque. Não restam dúvidas de que as culturas irrigadas resultam em produtividade maior e mais regular, já que a disponibilidade de água independe do regime de chuvas. Porém, a relação custo-benefício dever ser reavaliada levando-se em consideração os riscos à saúde do homem.

SERVICE (1989) destaca o arroz como principal cultura irrigada cerca de 140 milhões de hectares de terra são destinados ao seu cultivo, sendo que $90 \%$ da produção e do consumo ocorrem na Ásia. Trata-se do principal item alimentar de mais de $60 \%$ da população mundial. A necessidade de inundar áreas para cultivo do produto por vários períodos do ano resulta na formação de criadouros 
de mosquitos quase permanentes. Mais de 40 vírus têm sido associados às áreas de cultivo de arroz, sendo o vírus da encefalite japonesa, transmitido pelo Culex tritaeniorhynchus, o mais importante. Além disso, nos arredores das plantações, outras espécies de importância médica têm encontrado condições favoráveis para proliferação, como Culex quinquefasciatus nos locais urbanizados, Anopheles culicifacies nos canais de irrigação, e Aedes aegypti e Aedes albopictus em recipientes artificiais.

A região do Vale do Ribeira, São Paulo, chamou a atenção de pesquisadores em meados dos anos 70, quando ocorreu epidemia de encefalite por vírus Rocio e, possivelmente, outros. $\mathrm{Na}$ mesma ocasião iniciou-se na região, experimentalmente, cultivo irrigado de arroz, o qual inspirou outras pesquisas, principalmente por causa do risco de transmissão de malária. Apesar desse impacto ambiental, a região apresenta características primitivas, constituindo-se em local bastante adequado para conhecimento de populações de culicídeos de ambientes naturais. Desta forma, várias pesquisas bio-ecológicas foram conduzidas em ambientes modificados e naturais e estão sumarizadas em FORATTINI e MASSAD (1998).

Ainda assim, não se pode dizer que os conhecimentos sobre a região estão esgotados. $O$ presente trabalho pretendeu acrescentar aos estudos anteriores aspectos ecológicos das populações de culicídeos, com especial atenção às espécies de interesse médico, em ambiente de mata primitiva, mata residual e área de cultivo irrigado de arroz. 


\section{OBJETIVO}

Comparar a fauna culicidiana em ambiente de mata primária, mata residual e área de cultivo irrigado de arroz na Região do Vale do Ribeira, São Paulo.

\section{Objetivos especificos}

- Análise da composição faunística quanto ao número de espécies (S) e quanto ao número de indivíduos $(\mathrm{N})$

- Análise de diversidade, no tempo e espaço

- Indicação das espécies dominantes em cada tipo de ambiente e análise de suas distribuições

- Análise de constância das espécies nos ambientes

- Análise de similaridade entre os ambientes quanto ao número de espécies

- Discussão sobre a distribuição de espécies de importância médica 


\section{CARACTERÍSTICAS DO VALE DO RIBEIRA}

A descrição a seguir baseia-se na publicação de FORATTINI e col. (1978a). A região conhecida como Vale do Ribeira situa-se entre $24^{\circ}$ e $25^{\circ} 16^{\prime}$ de latitude sul e $46^{\circ} 50^{\prime}$ e $49^{\circ} 20^{\prime}$ de longitude oeste, compreendendo duas unidades: litoral, que constitui-se por praias e baixadas; e Serra do Mar, constituída por escarpas da borda ocidental do planalto cristalino interiorano. Esta unidade, que limita o litoral, estendendo-se por mais de $1000 \mathrm{~km}$ desde o Estado de Santa Catarina até o do Rio de Janeiro, apresenta altitudes variáveis entre 800 e 1800 metros.

No Estado de São Paulo, o Vale do Ribeira pode ser dividido em três regiões, em relação ao relevo: a baixada - possui cerca de $2400 \mathrm{~km}^{2}$ e forma a planície litorânea tropical, onde geralmente ficam os núcleos urbanos locais; a escarpa da serra - possui cerca de $8600 \mathrm{~km}^{2}$ orientados no sentido nordeste-sudoeste, aproximando-se da costa ao norte e ao sul; e a região pré-serrana, com características de transição entre as duas anteriores.

No planalto cristalino, o clima é temperado, subtropical ou mesotérmico brando superúmido, sem estação seca e com temperatura média de $22^{\circ} \mathrm{C}$ no mês mais quente (janeiro). $\mathrm{Na}$ baixada litorânea, o clima é tropical úmido ou superúmido, sem estiagem (mais de $60 \mathrm{~mm}$ de chuva no mês mais seco - julho) e com temperatura média superior a $22^{\circ} \mathrm{C}$ (janeiro). Na parte paulista do vale, o clima é subtropical (no interior) e tropical (na costa), superúmido, com precipitação média anual de $1800 \mathrm{~mm}$, distribuídos ao longo de 175 dias no ano. Na escarpa da Serra, as chuvas podem atingir valores entre 2000 e $3000 \mathrm{~mm}$. A temperatura média é superior a $22^{\circ} \mathrm{C}$ no mês mais quente, podendo alcançar valores de 35 a $40^{\circ} \mathrm{C}$ na planície, área de influência da massa tropical atlântica. No mês mais frio a temperatura média é de $13^{\circ} \mathrm{C}$. A atuação da massa polar pode reduzir esse valor a $3^{\circ} \mathrm{C}$ na planície e $0^{\circ} \mathrm{C}$ na escarpa.

Em relação à fitogeografia local, predomina o aspecto florestal de mata perenifólia higrófila (floresta latifoliada tropical úmida da encosta ou floresta perenifólia latifoliada higrófila costeira), que pode ser considerada clímax. Essa formação apresenta indivíduos arbóreos de porte elevado (até 30 a $40 \mathrm{~m}$ de altura), aspecto denso e alto grau de heterogeneidade. Destaca-se a riqueza de bromeliáceas 
epifitas, terrestres e rupestres em toda a região. Nas áreas mais elevadas a vegetação estratifica-se em três andares e, na baixada, em dois. Ao sul do vale ocorrem feições de planalto de araucárias. $\mathrm{Na}$ faixa costeira a vegetação é rasteira, do tipo pantanoso marinho dos mangues e cobertura arbórea-arbustiva de indivíduos de porte médio, conhecida como "restinga" ou "jundu". Nas áreas da baixada e pré-serrana encontram-se formações hidrófitas, que acompanham os cursos de água nos locais alagados da planície.

O Vale do Ribeira vem sofrendo modificações desde a época do descobrimento do Brasil, principalmente nas matas da planície, por serem as mais acessíveis. Deste modo, entende-se por floresta ou mata primária as áreas que sofreram pouca atividade antrópica, conservando características primitivas e, por floresta ou mata secundária, áreas que sofreram alterações nas suas características primitivas. 


\section{LOCALIDADES ESTUDADAS}

O presente estudo refere-se aos ambientes de mata primária, mata residual e cultivo irrigado de arroz. Os dois últimos localizam-se na Fazenda Experimental de Pariquera-Açu (fig.1), cuja descrição baseia-se em FORATTINI e col. (1981, 1989a, 1993b).

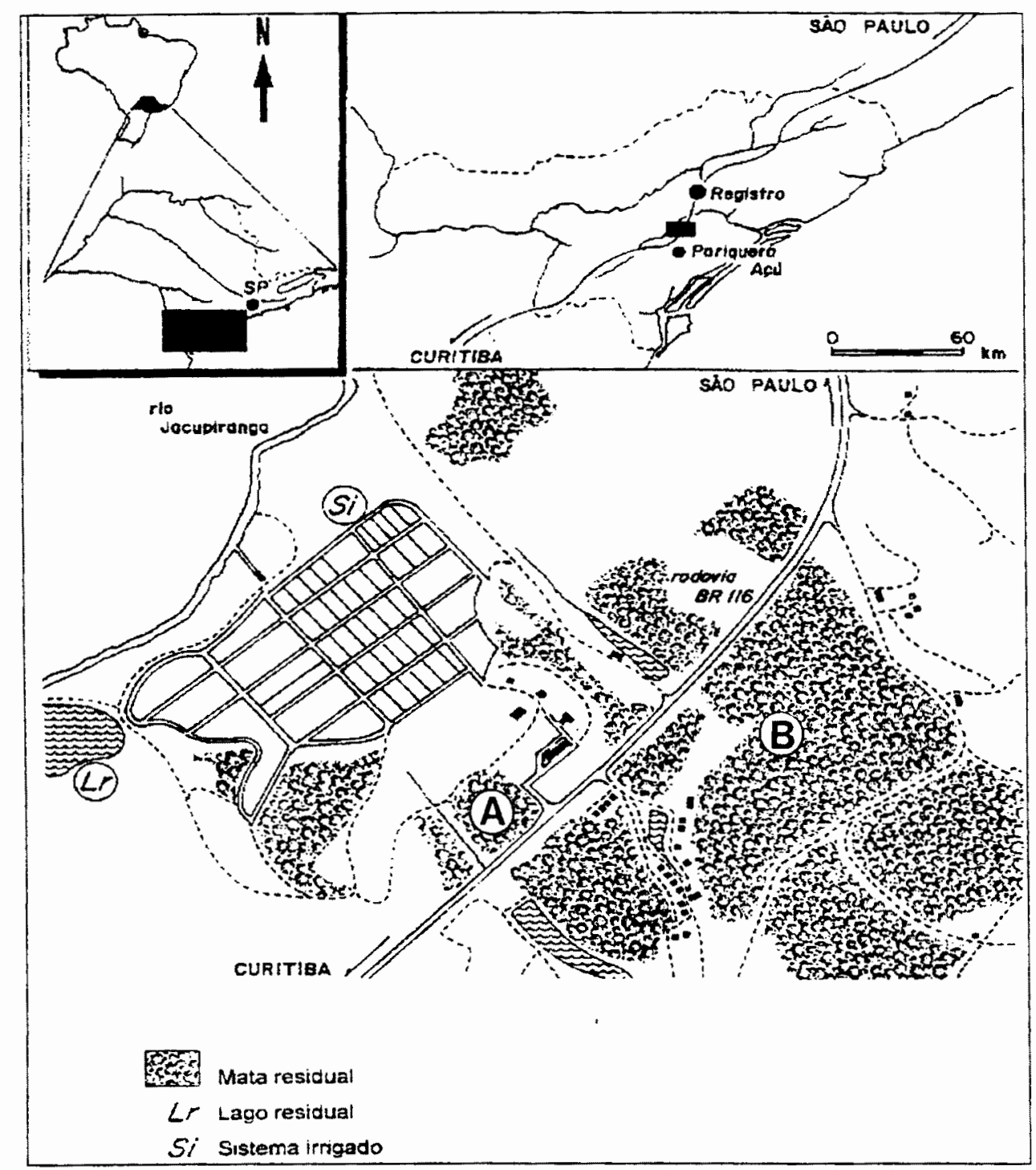

Fig.1: Representação da Fazenda Experimental do Instituto Agronômico de Campinas em Pariquera-Açu (SP), onde foram adotadas as localidades de mata residual (A e B) e área de cultivo irrigado de arroz (Si). 
A Fazenda Experimental de Pariquera-Açu (Estação Experimental do Vale do Ribeira), pertencente ao Instituto Agronômico de Campinas - Secretaria do Estado de São Paulo, possui 256 hectares e localiza-se na altura do quilòmetro 200 da rodovia Regis Bittencourt (BR-116), ligando São Paulo a Curitiba, o que corresponde a $24^{\circ} 30^{\prime}$ de latitude sul e $47^{\circ} 50^{\prime}$ de longitude oeste. A precipitação média anual é de $2250 \mathrm{~mm}$, as temperaturas médias oscilam entre 18 e $25^{\circ} \mathrm{C}$ e a umidade relativa do ar, constante, é de aproximadamente $80 \%$.

Desde 1978, cerca de 11 hectares são utilizados para cultura experimental de arroz irrigado, também denominado de arroz transplantado. O ciclo do arroz envolve três etapas: preparo do solo, transplante das mudas e colheita. A primeira etapa inicia novo ciclo, após três meses de descanso do solo, e consiste na aragem e gradagem do terreno, que é inundado para nivelamento. A seguir, pesticidas são aplicados $\mathrm{e}$ as mudas são transplantadas. $O$ terreno fica permanentemente inundado até o início da formação dos primórdios florais. A partir de então, as inundações passam a ser intermitentes até a maturação, que coincide com o fim de março; logo em seguida, é feita a colheita (terceira etapa). O terreno permanece sem água, exceto a proveniente das chuvas, até o início de novo experimento. Portanto, nos terrenos irrigados para cultivo, ocorre alternância de períodos de seca e inundação.

As áreas de mata residual da fazenda apresentam grau variável de características primárias. Para o estudo foram escolhidas duas áreas - mata A e B com características mais primitivas, ou seja, com predomínio de árvores de porte elevado.

Os ambientes de mata primária - "Fonte" (fig.2) e sítio Gentil (fig.3) situam-se no município de Cananéia. As caracteristicas correspondem às da mata perenifólia da encosta, conforme descrito na caracterização do Vale do Ribeira. 


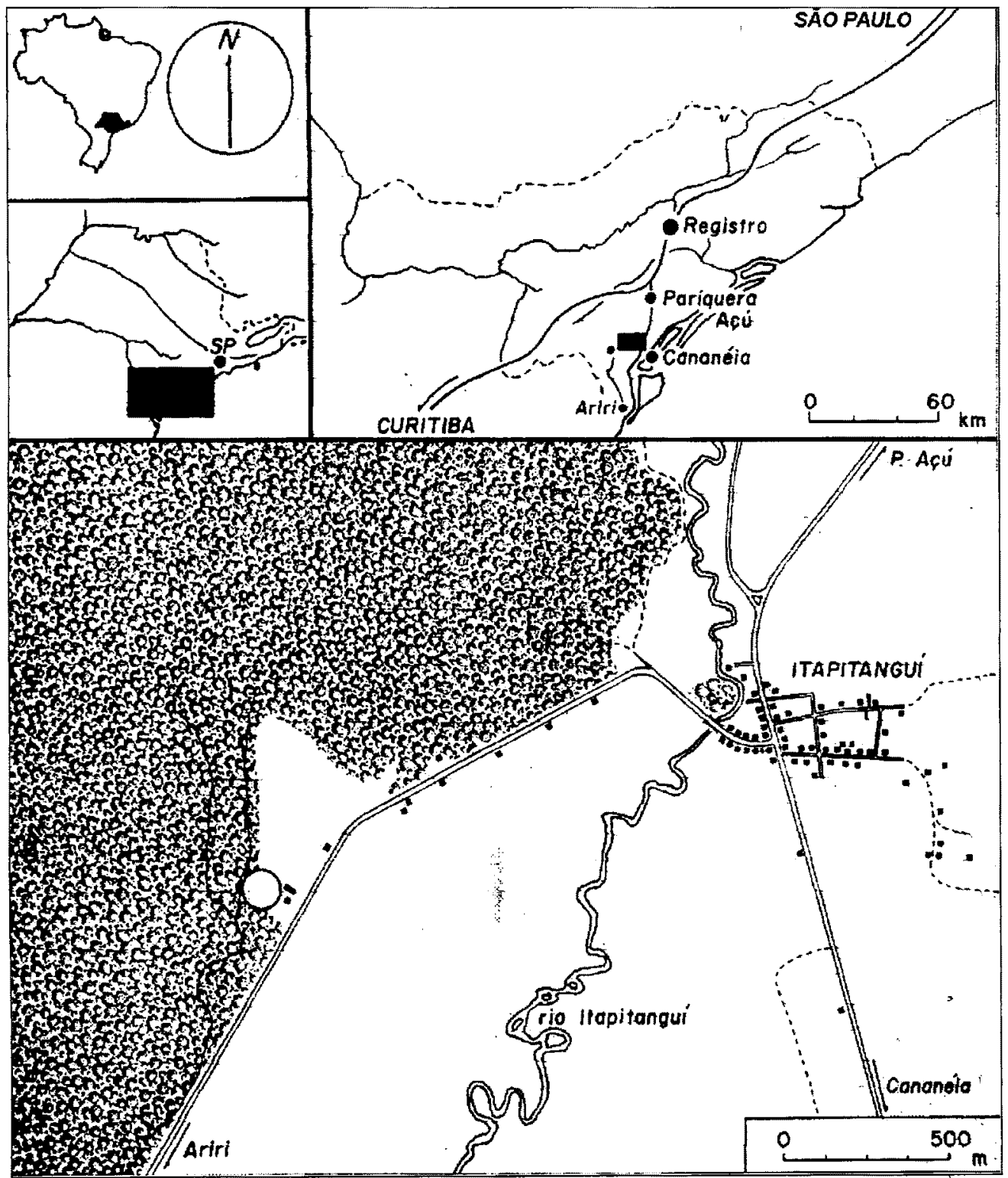

Fig.2: Representação de área de mata primária - "Fonte", em Cananéia (SP). As coletas foram efetuadas no ponto assinalado. 


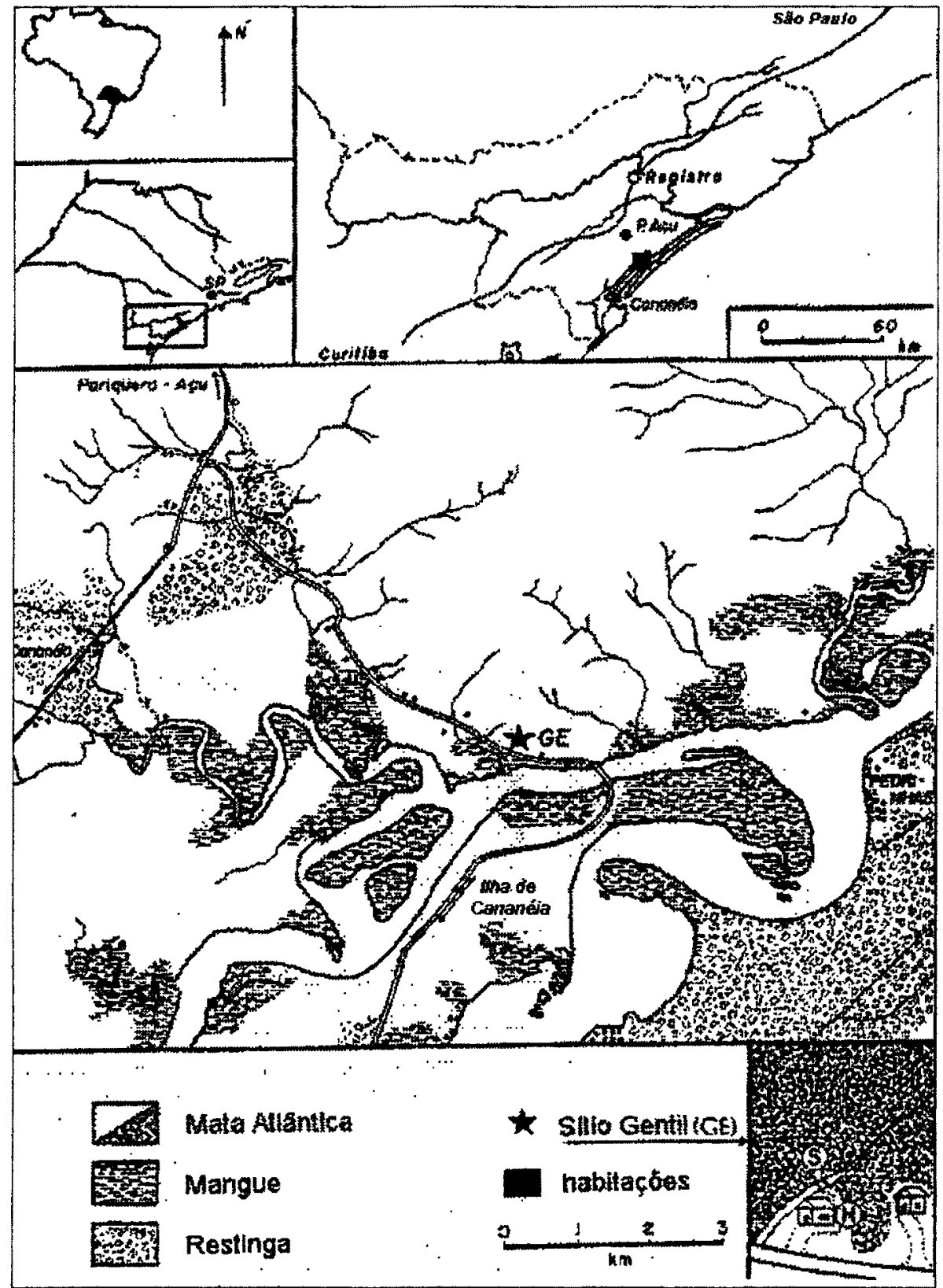

Fig.3: Representação de área de mata primária - Sítio Gentil, em Cananéia (SP). 


\section{MATERIAL E MÉTODO}

Este trabalho utiliza os dados do Projeto Temático "Culicidae do agroecossitema irrigado e seu significado epidemiológico" (FAPESP, Processo 90/33716), desenvolvido entre os anos de 1991 e 1995, a partir do qual foram publicados vários trabalhos sobre o significado ecológico e epidemiológico de culicídeos em ambiente antrópico, os quais estão sintetizados em FORATTINI e MASSAD (1998). Particularmente, foram utilizados dados referentes às formas adultas de culicídeos coletadas em área de mata, primária e residual, e arrozal, durante o período de janeiro de 1992 a abril de 1995, utilizando-se como técnica de captura armadilha tipo Shannon, com fonte luminosa e dois coletores com aspirador manual. As coletas foram realizadas quinzenalmente, das 17:00 às 22:00 horas, porém os dados aqui são apresentados mensalmente.

O período de coletas variou de um local para outro, a saber: na "Fonte", área de mata primária, fevereiro de 1992 a janeiro de 1993; no Sítio Gentil, também área de mata primária, junho de 1993 a abril de 1995; na mata A, área de mata residual, janeiro de 1992 a dezembro de 1994; na mata B, também área de mata residual, fevereiro de 1992 a dezembro de 1994; na área de cultivo irrigado de arroz, as coletas foram realizadas de janeiro de 1992 a dezembro de 1993.

Para análise da composição faunística quanto ao número de espécies (S) e quanto ao número de indivíduos $(\mathrm{N})$ foram traçados gráficos referentes ao número acumulado de espécies e de indivíduos para cada ambiente a cada ano (ainda que as coletas tenham ocorrido apenas em alguns meses) bem como para os períodos totais de captura.

Para estudo da diversidade de culicídeos foram construídos gráficos para ilustrar o padrão de equidade. Além disso, foi adotado o Índice de Diversidade de Margalef (d) (MAGURRAN 1988; SERVICE 1993; HAYEK e BUZAS 1997), indice de Margalef padronizado (d/dmáx) (HAYEK e BUZAS 1997) e o índice de Fisher ( $\alpha$ ) (DAJOZ 1973; MAGURRAN 1988; SERVICE 1993; HAYEK e BUZAS 1997). Os índices foram calculados para cada ano nos três ambientes e também levando-se em consideração o periodo total de captura. 
Índice de Margalef $=\mathrm{d}=(\mathrm{S}-1) / \mathrm{ln} \mathrm{N}$, onde

$\mathrm{S}=$ número de espécies

$\mathrm{N}=$ soma do número de individuos de todas as espécies

Índice de Margalef Padronizado

$\mathrm{d} / \mathrm{dmáx}=[(\mathrm{S}-1) / \ln \mathrm{N}] /[(\mathrm{N}-1) / \ln (\mathrm{N})]$

$\mathrm{S}=$ número de espécies

$\mathrm{N}$ = soma do número de indivíduos de todas as espécies

Índice de Fisher

$\alpha=N(1-x) / x$, onde $x$ pode ser estimado pela equação

$\mathrm{S} / \mathrm{N}=(1-\mathrm{x}) / \mathrm{x}[-\ln (1-\mathrm{x})]$, sendo

$\mathrm{S}=$ número de espécies

$\mathrm{N}$ = soma do número de indivíduos de todas as espécies

$\operatorname{Var}(\alpha)=\alpha /-\ln (1-x)$ (MAGURRAN 1988; SERVICE 1993)

$\operatorname{Var}(\alpha)=\left\{\alpha^{3}\left[(\mathrm{~N}+\alpha)^{2} \ln (2 \mathrm{~N}+\alpha / \mathrm{N}+\alpha)-\alpha \mathrm{N}\right\} /(\mathrm{SN}+\mathrm{S} \alpha-\mathrm{N} \alpha)^{2}\right.$

(HAYEK e BUZAS 1997)

Os intervalos de confiança de $\alpha$ foram estabelecidos ao nível de $95 \%$, conforme SERVICE (1993).

Para a indicação de espécies dominantes foi adotado o Índice de Berger-Parker (MAGURRAN 1988).

Indice de Berger-Parker $=\mathrm{d}_{\mathrm{BP}}=\mathrm{N}_{\text {máx }} \cdot / \mathrm{N}$, onde

$\mathrm{N}_{\text {máx }}=$ número de indivíduos da espécie mais abundante

$\mathrm{N}=$ soma do número de indivíduos de todas as espécies 
A distribuição da espécie dominante em cada local apresenta-se em paralelo com a distribuição dos valores de precipitação mensal. Estes provêm do banco de dados de medidas de precipitação do Departamento de Águas e Energia Elétrica (DAEE). As medidas referentes às localidades de mata primária foram feitas em Cananéia e às demais em Pariquera-Açu. Além disso, efetuou-se o cálculo do coeficiente de Pearson para inverstigar possível correlação entre a distribuição de frequências das espécies dominantes e as medidas de precipitação, tanto do mês correspondente quanto do mês anterior.

A constância das espécies nos ambientes foi feita segundo o índice $c$ (DAJOZ 1973), para cada ambiente, segundo os períodos totais de captura.

$\mathrm{c}=\mathrm{p}(100) / \mathrm{P}$, onde

$\mathrm{p}=$ número de coletas contendo a espécie estudada

$\mathrm{P}=$ número total de coletas efetuadas

Em função do valor de c distinguem-se as categorias:

Espécies constantes ( $\mathrm{x}$ ) - presentes em mais de $50 \%$ das coletas

Espécies acessórias (y) - presentes em 25 a 50\% das coletas

Espécies acidentais (z) - presentes em menos de $25 \%$ das coletas

A análise de similaridade entre os ambientes quanto ao número de espécies foi feita segundo o indice de Sorensen (MAGURRAN 1988; SERVICE 1993)

$\mathrm{C}_{\mathrm{s}}=2 \mathrm{j} /(\mathrm{a}+\mathrm{b})$, onde

$\mathrm{j}=$ número de espécies encontradas nos dois locais

$\mathrm{a}=$ número de espécies no local $\mathrm{A}$

$\mathrm{b}=$ número de espécies no local $\mathrm{B}$

$\mathrm{O}$ valor desse índice pertence ao intervalo de zero a 1 , indicando grau mínimo e máximo de similaridade. 


\section{RESULTADOS}

\section{Número de Espécies (S) e Número de Indivíduos (N)}

\subsection{S e N em ambiente de Mata Primária}

Os dados apresentam-se aqui sob a forma de gráficos. Nas tabelas 19 a 27 do apêndice estão os valores dos pontos plotados.

Nas áreas de mata primária - "Fonte" e Sítio Gentil (Cananéia, SP) foram capturados ao todo 37383 e 26590 mosquitos, pertencentes a 94 e 86 espécies, respectivamente. É importante lembrar que os dados da "Fonte" referem-se a 12 meses de coleta (fev/1992 a jan/1993), enquanto que no Sítio Gentil, 23 meses (jun/1993 a abril/1995).

A fig.4 apresenta o número acumulado de espécies na "Fonte". Notase que nas duas primeiras capturas são acrescentadas mais de 20 espécies. $\mathrm{Na}$ seqüência, os acréscimos são pequenos, fazendo com que a curva praticamente se estabilize, voltando a ascender ligeiramente em outubro, novembro e dezembro de 1992. A média mensal do número de espécies foi $30 \pm 9$.

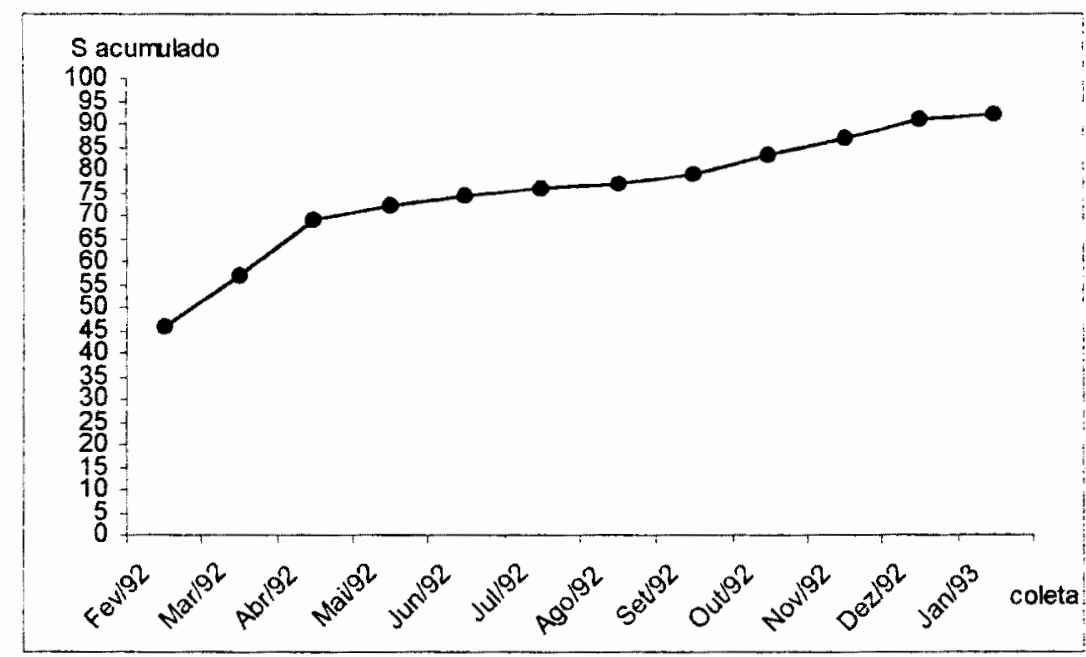

Fig.4: Número acumulado de espécies coletadas em armadilha tipo Shannon, em área de mata primária ("Fonte" - Cananéia, SP) no periodo de 12 meses. 
A fig. 5 apresenta o número acumulado de individuos ( $\mathrm{N}$ acumulado) a cada coleta na "Fonte" e mostra que, de um modo geral, o número de indivíduos teve correspondência com o número de espécies. Destacou-se a captura de fevereiro, que contribuiu com mais de $25 \%$ do total de indivíduos coletados durante o período de 12 meses, com 9646 indivíduos. Destacaram-se também as coletas de junho e outubro, que acrescentaram cerca de $10 \%$ do total ( 4016 e 3882 , respectivamente), bem como o mês de dezembro, com 15\% (5740 indivíduos). O N total foi 37383 e o médio, $3115 \pm 2451$.

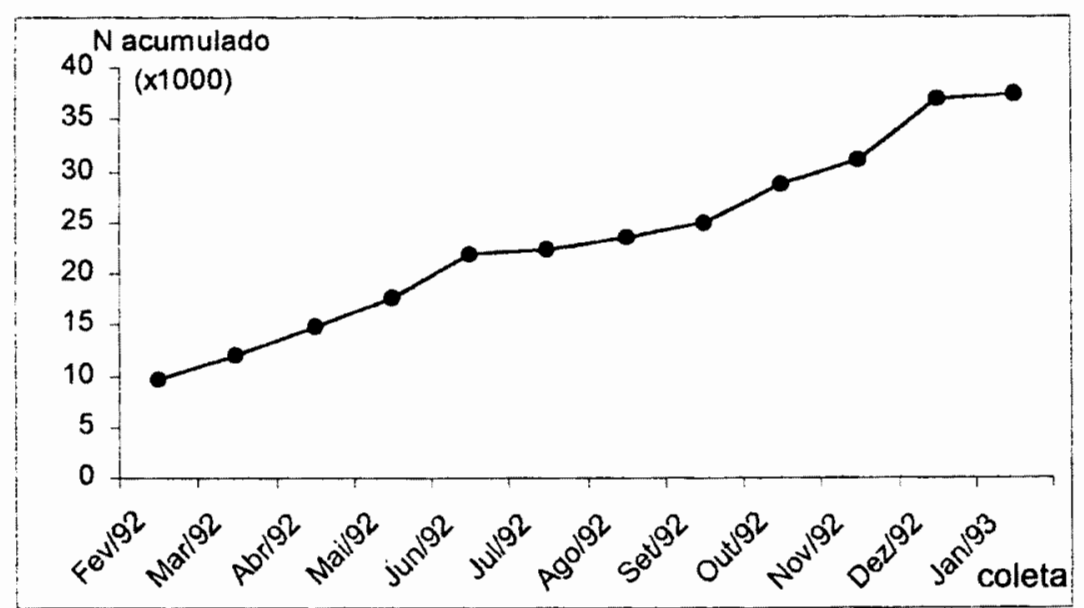

Fig.5: Número acumulado de individuos coletados em armadilha tipo Shannon em área de mata primária ("Fonte" - Cananéia, SP) no periodo de 12 meses.

A seguir, as figuras 6 e 7 apresentam, respectivamente, os dados do número acumulado de espécies e de indivíduos coletados no Sítio Gentil a cada ano, para os periodos correspondentes de captura. Em 1993 foram sete meses (junho a dezembro), totalizando 43 espécies e 7530 individuos. O maior valor de $\mathrm{S}$ foi 26 e $o$ de $\mathrm{N}$ foi 2647 , ambos referentes à coleta de novembro. Os valores mínimos foram $\mathrm{S}=8$ (julho e agosto) e $\mathrm{N}=267$ (agosto). $O$ número médio de espécies foi $15 \pm 5$ e de individuos foi $1075 \pm 868$.

Em 1994, o S acumulado foi 71 e o $\mathrm{N}$ acumulado, 10860. Neste ano não houve correspondência dos maiores valores de $\mathrm{S}$ e $\mathrm{N}$; o maior $\mathrm{S}$ foi 29 , em janeiro, quando foram coletados 758 mosquitos. O maior N, 1892, ocorreu em novembro ( $S=23$ ). Os menores valores de $S$ e $\mathrm{N}$ correspondem ao mês de julho, com 
45 individuos pertencentes a 10 espécies. O $\mathrm{N}$ médio foi de $905 \pm 685$ e o $\mathrm{S}$ médio, $20 \pm 6$.

Nos quatro meses de captura em 1995 (janeiro a abril), o número acumulado de espécies foi de 59 , entre as quais distribuíram-se 8200 mosquitos. Em janeiro ocorreu o menor $S$, igual a 21 e o menor $N$, igual a 2274. Em março, os maiores valores: $S=46$ e $N=2556$. Médias de 30 espécies $( \pm 9)$ e 2050 indivíduos $( \pm 656)$.

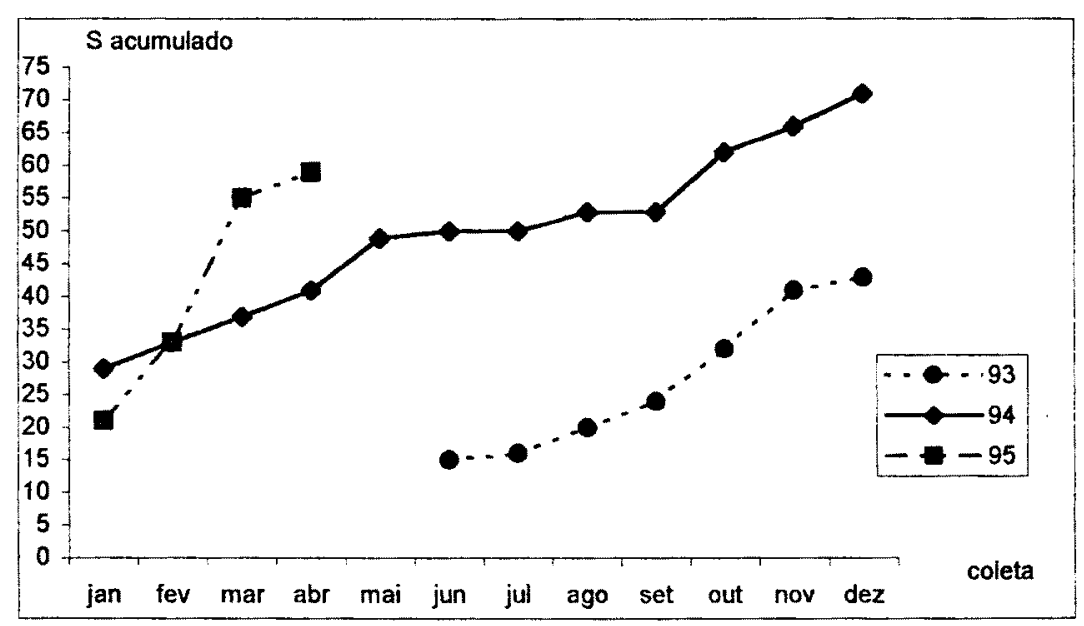

Fig.6: Número acumulado de espécies coletadas em armadilha tipo Shannon, em área de mata primária ("Sítio Gentil" - Cananéia, SP) levando-se em consideração períodos de até um ano.

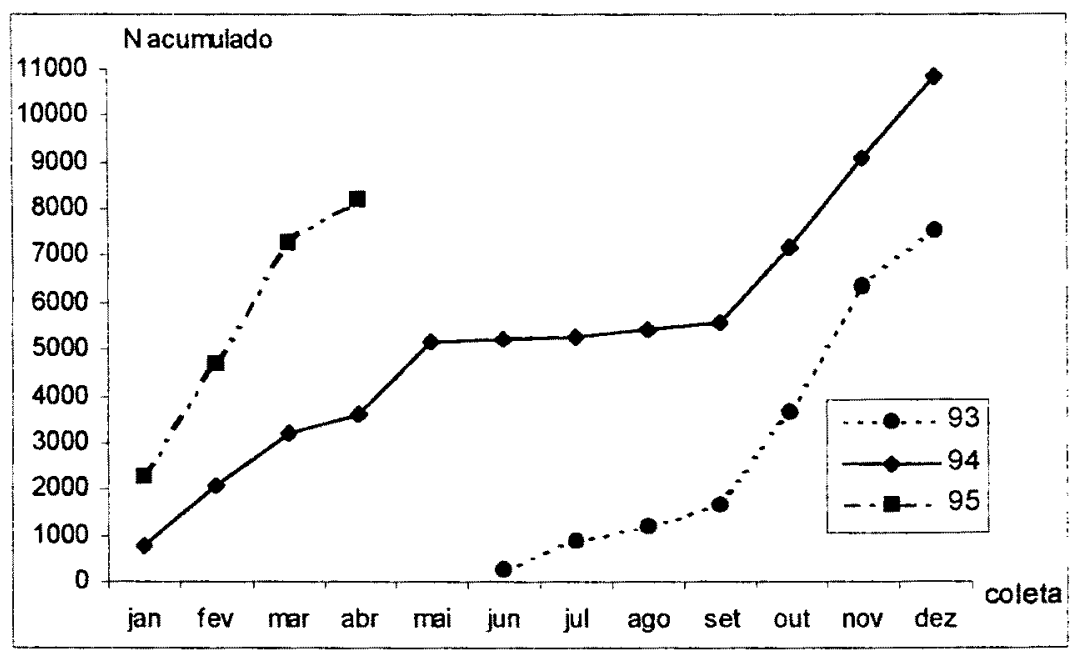

Fig.7: Número acumulado de indivíduos coletados em armadilha tipo Shannon em área de mata primária ("Sítio Gentil - Cananéia, SP) levando-se em consideração periodos de até um ano de captura. 
A fig. 8 traz os dados de número acumulado de espécies no Sítio Gentil considerando-se o período de junho de 1993 a abril de 1995. O número de espécies a cada mês variou entre 8 e 46 (média $20 \pm 8$ ) e, na maioria das vezes, o número de espécies adicionadas foi inferior a 5 , fazendo com que a curva ascendesse continuamente de 15 a 86 espécies.

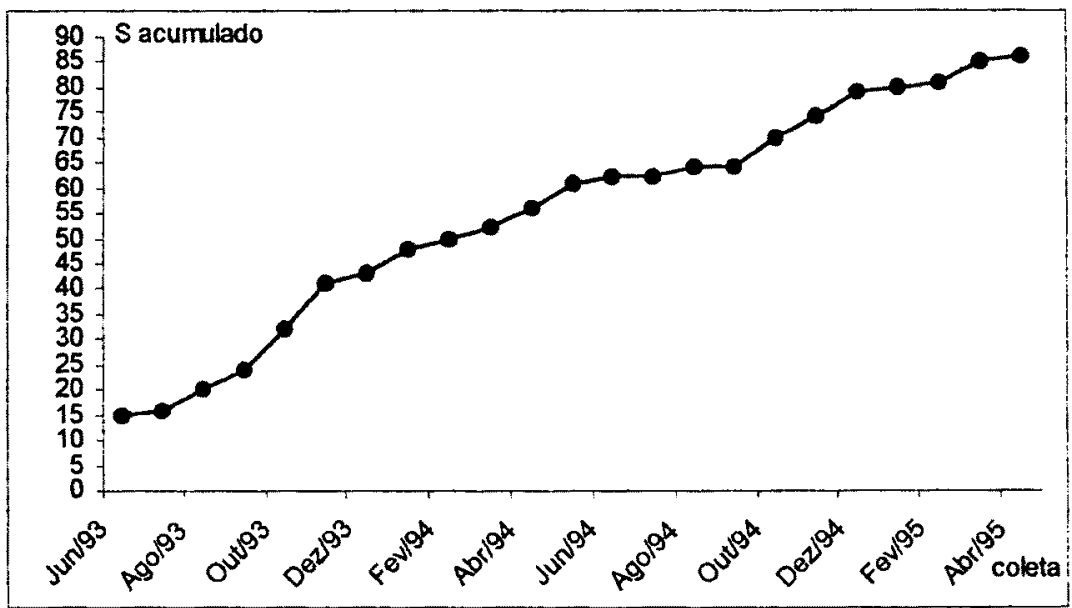

Fig.8: Número acumulado de espécies coletadas em armadilha tipo Shannon, em área de mata primária ("Sítio Gentil" - Cananéia, SP) durante 23 meses de captura.

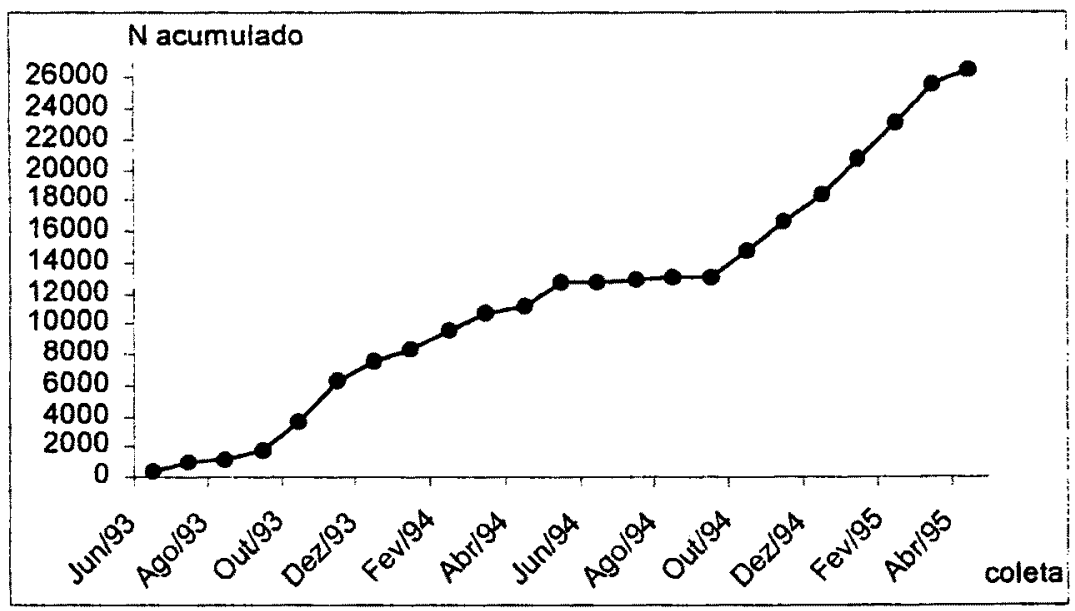

Fig.9: Número acumulado de indivíduos coletados em armadilha tipo Shannon, em área de mata primária ("Sítio Gentil" - Cananéia, SP) durante 23 meses de captura. 
A fig.9, referente ao número acumulado de indivíduos na mesma localidade e período, apresenta curva que inicia-se com 246 e termina com 26590 mosquitos ( $\mathrm{N}$ médio $=1156 \pm 850$ ). Entre junho e setembro, a curva apresenta acréscimos pequenos (45 a 655 indivíduos); de outubro de 1993 e março de 1994 e de outubro de 1994 a março de 1995 a curva ascendeu de forma mais acentuada, com adição de cerca de 1800 indivíduos.

\subsection{S e $\mathrm{N}$ em ambiente de Mata Residual}

Nos pontos de mata residual - mata A e mata B (Pariquera-Açu, SP) foram coletados, respectivamente, 28915 mosquitos pertencentes a 120 espécies e 19033 mosquitos de 113 espécies. Na mata A o período de estudos foi de janeiro de 1992 a dezembro de 1994 e na mata B, de fevereiro de 1992 a dezembro de 1994.

As figuras 10 e 11 trazem as curvas do número acumulado de espécies e de individuos na mata $\mathrm{A}$, considerando-se cada ano de coleta.

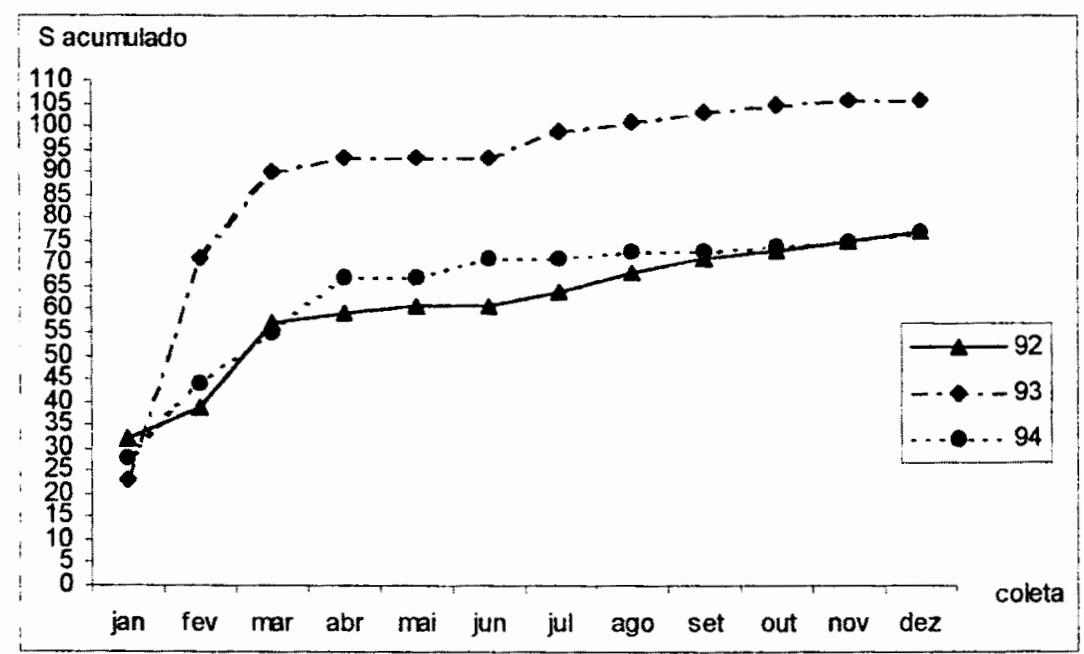

Fig. 10: Número acumulado de espécies coletadas em armadilha tipo Shannon em área de mata residual ("mata A" - Fazenda Experimental do IAC - Pariquera-Açu, SP) levando-se em consideração cada ano de captura. 
Em relação ao número de espécies, as curvas referentes aos anos de 1992 e 1994 quase se sobrepõem, acumulando 79 e 77 espécies (S médio $25 \pm 10$ e $27 \pm 7$, respectivamente). Para os três anos a curva ascende nos três primeiros meses (no caso de 1994, até abril) e depois praticamente se estabiliza, embora tenham sido adicionadas 2 espécies em dezembro de 1992 e 1994. O ano de 1993 destacou-se dos demais, acumulando 106 espécies ( $\mathrm{S}$ médio $32 \pm 15$ ). Tal destaque deve-se ao mês de fevereiro, que adicionou 48 espécies, contrastando com 7 em 1992 e 16 em 1994.

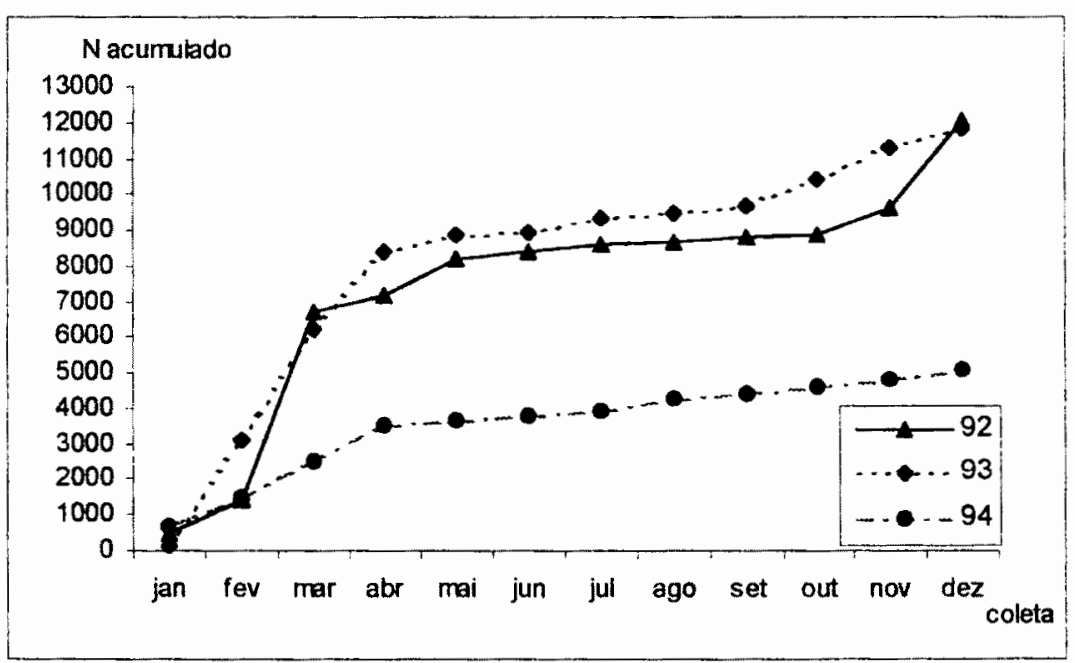

Fig.11: Número acumulado de individuos coletados em armadilha tipo Shannon em área de mata residual ("mata A" - Fazenda Experimental do IAC - Pariquera-Açu, SP) levando-se em consideração cada ano de captura.

Em relação ao número de indivíduos (fig.11), a curva que se destaca é a de 1994, que acumula um total de 5075 mosquitos ( $N$ médio $422 \pm 335$ ), enquanto que as curvas de 1992 e 1993 apresentam-se bastante semelhantes, acumulando 12023 e 11817 indivíduos, respectivamente ( $N$ médio $1001 \pm 1430$ e $984 \pm 1059$ ).

Quando se considera o período de janeiro de 1992 a dezembro de 1994, o número acumulado de espécies estabiliza-se no valor 120 (S médio $28 \pm 11$ ), em agosto de 1994. A partir dai nenhuma espécie foi adicionada (fig.12). 


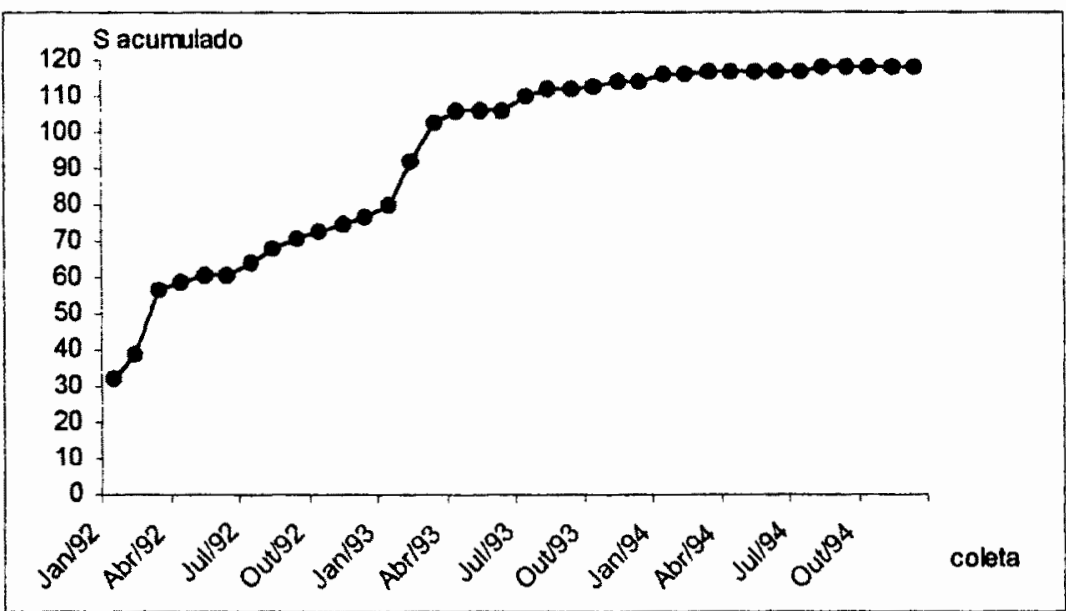

Fig.12: Número acumulado de espécies coletadas em armadilha tipo Shannon em mata residual ("mata A" - Fazenda Experimental do IAC - Pariquera-Açu, SP) durante o periodo de janeiro de 1992 a dezembro de 1994.

Quanto ao número de individuos (fig.13), a curva sobe acentuadamente no mês de março de 1992, com 5266 mosquitos; outras capturas importantes foram as de dezembro de 1992 e fevereiro a abril de 1993, com cerca de 2500 indivíduos. As demais contribuíram com menos de 1000 indivíduos ( $\mathrm{N}$ médio $803 \pm 1079$ ).

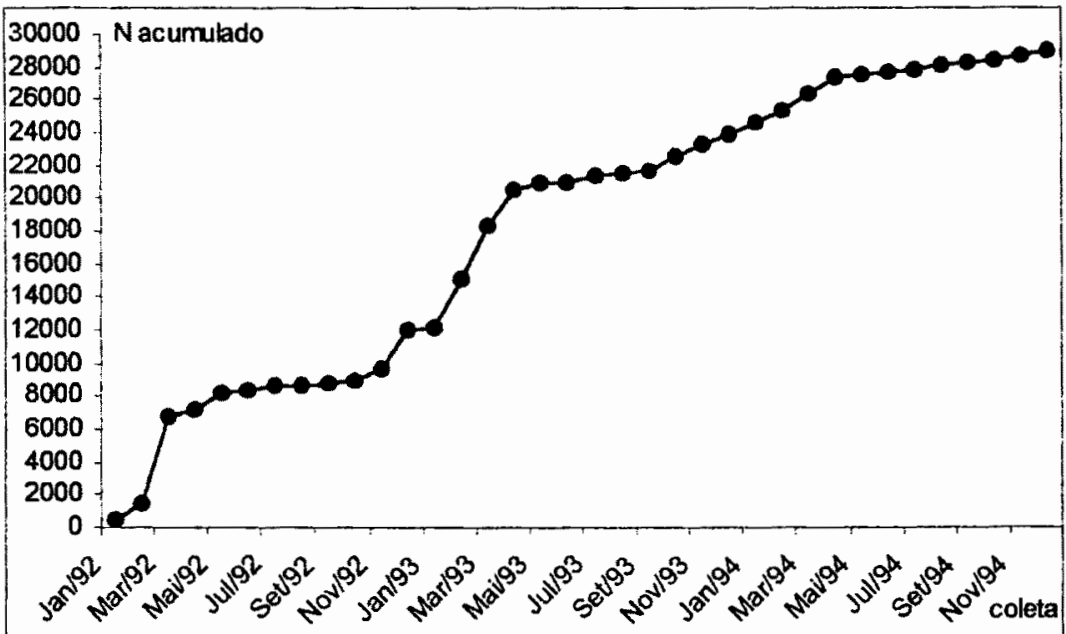

Fig.13: Número acumulado de individuos coletados em armadilha tipo Shannon em área de mata residual ("mata A" - Fazenda Experimental do IAC - Pariquera-Açu, SP) durante o período de janeiro de 1992 a dezembro de 1994. 
Na mata $\mathrm{B}$, a curva do número de espécies (fig.14) que se destaca é a referente ao ano de 1992, que apresenta o mesmo perfil das outras, porém com

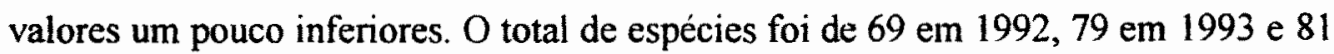
em 1994. O número médio de espécies foi $21 \pm 8,27 \pm 11$ e $24 \pm 9$, respectivamente. Os maiores acréscimos de espécies ocorreram nos primeiros meses, mas para os três anos o mês de dezembro ainda adicionou 1 espécie em 1992, 2 em 1993 e 5 em 1994.

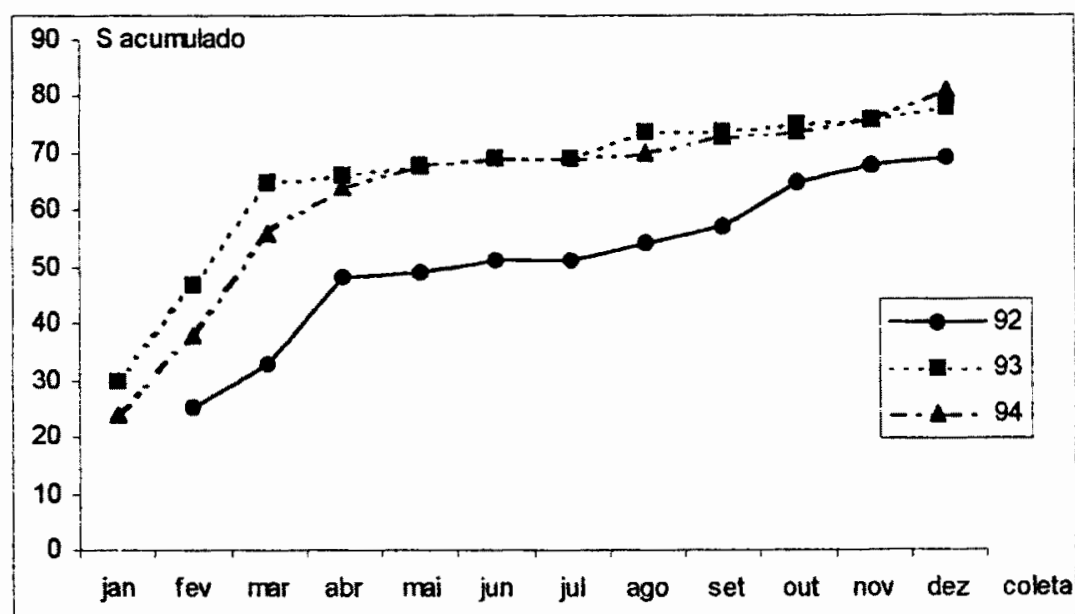

Fig. 14: Número acumulado de espécies coletadas em armadilha tipo Shannon em área de mata residual ("mata B" - Fazenda Experimental do IAC - Pariquera-Açu, SP) levando-se em consideração cada ano de captura.

A fig. 15, a seguir, mostra que o número de indivíduos foi variável de ano a ano, embora 1992 e 1994 tenham acumulado totais semelhantes: 4143 e 4683, respectivamente. 1993 apresentou valores sempre maiores que 1992 e 1994, acumulando, ao todo, 10207 individuos. A média foi de $376 \pm 227$ individuos em $1992,850 \pm 601 \mathrm{em} 1993$ e $390 \pm 378$ em 1994. 


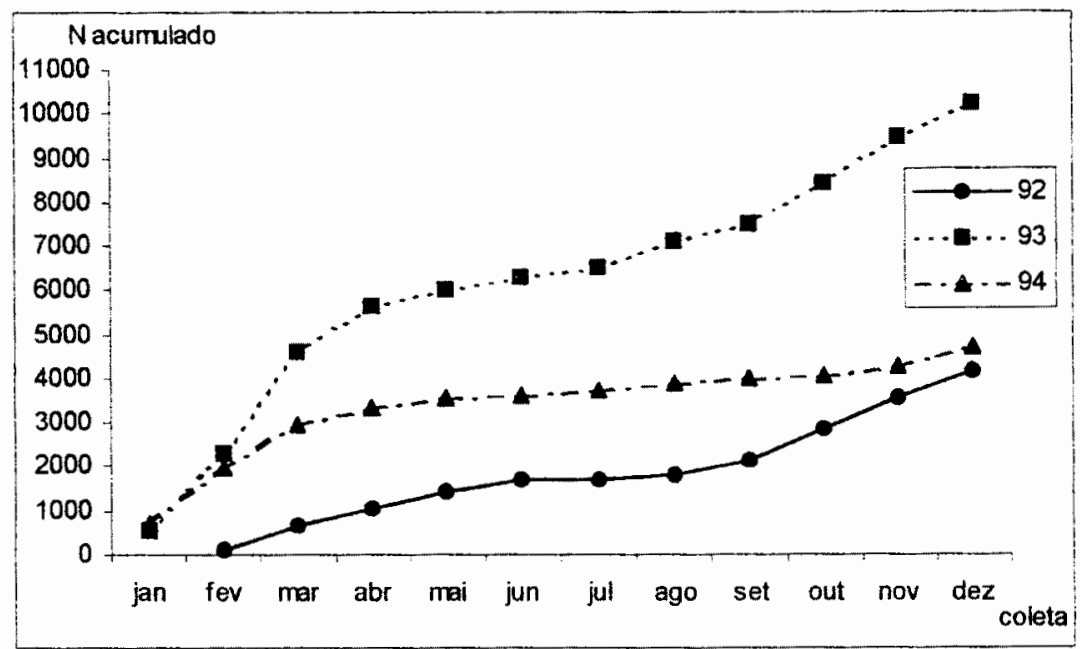

Fig.15: Número acumulado de indivíduos coletados em armadilha tipo Shannon em área de mata residual ("mata B" - Fazenda Experimental do IAC - Pariquera-Açu, SP) levando-se em consideração cada ano de captura.

$\mathrm{Na}$ fig. 16 observa-se que a curva do número acumulado de espécies não se estabiliza, quando se considera o periodo de fevereiro de 1992 a dezembro de 1994. Na coleta de dezembro de 1994 foram adicionadas 4 espécies, totalizando 113. Destaque para os meses de abril de 1992 e março de 1993, que adicionaram 15 e 11 espécies, respectivamente (S médio $24 \pm 9$ ).

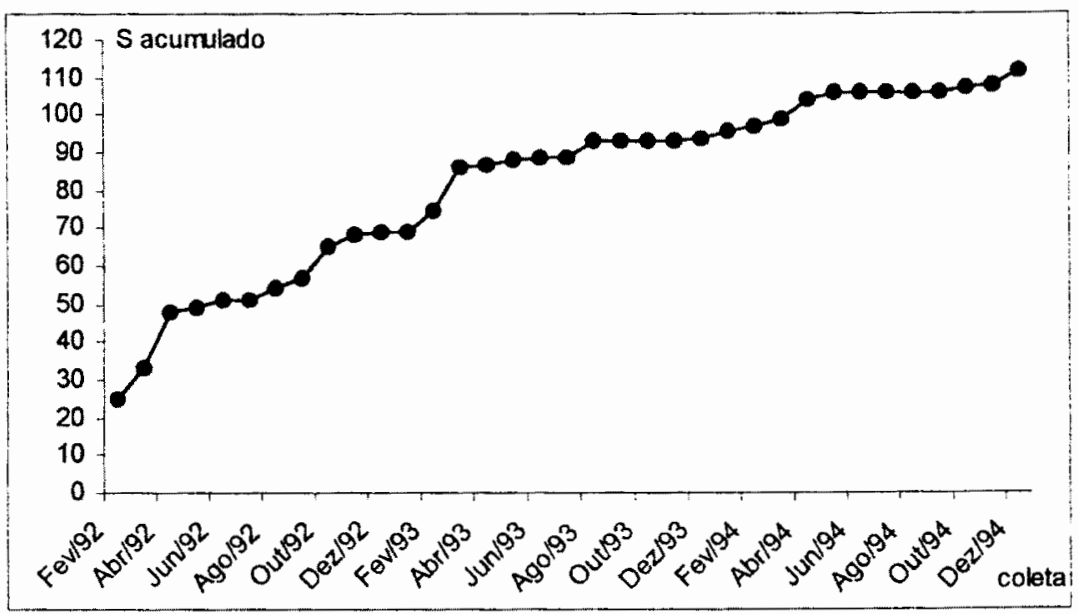

Fig.16: Número acumulado de espécies coletadas em armadilha tipo Shannon em área de mata residual ("mata B" - Fazenda Experimental do IAC - Pariquera-Açu, SP) durante o período de fevereiro de 1992 a dezembro de 1994. 
Quanto ao número de indivíduos, a fig. 17 mostra que a curva acumula 19033 mosquitos. Os valores mensais de N não passaram de 800 em 1992. Em 1993 os valores se elevaram, chegando a 2325 (março) e em fevereiro e março de 1994, o $\mathrm{N}$ foi superior a 1000 . O $\mathrm{N}$ médio do periodo todo foi de $543 \pm 488$ indivíduos.

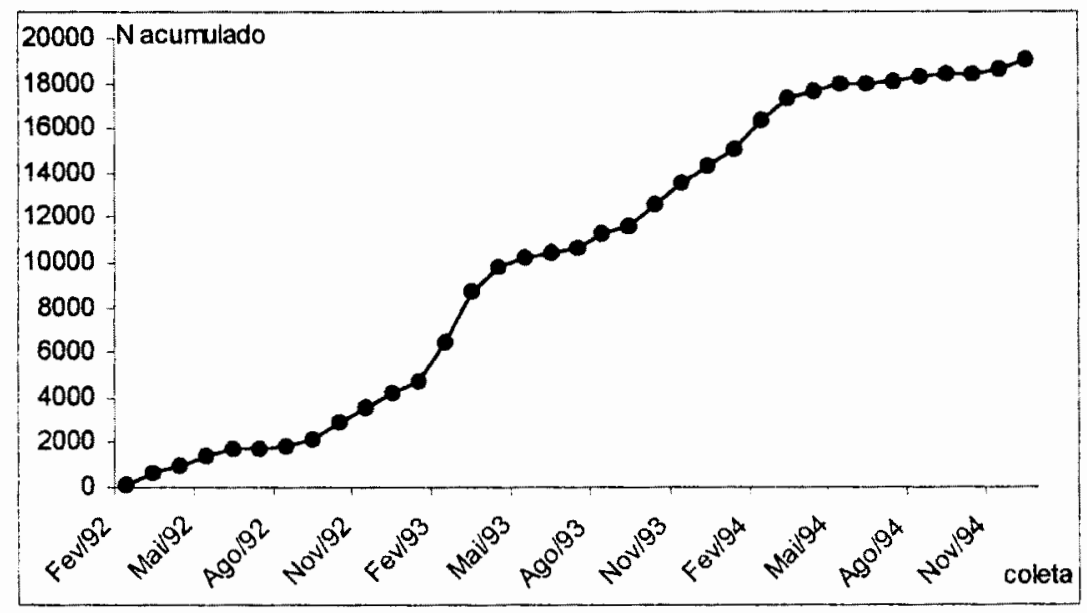

Fig.17: Número acumulado de indivíduos capturados em armadilha tipo Shannon em área de mata residual ("mata B" - Fazenda Experimental do IAC - Pariquera-Açu, SP) durante o período de fevereiro de 1992 a dezembro de 1994.

\subsection{S e $\mathbf{N}$ em área de cultivo irrigado de arroz}

A área de cultivo irrigado de arroz pertence à Fazenda Experimental do Instituto Agronômico de Campinas (Pariquera-Açu, SP), onde foram realizadas coletas durante 1992 e 1993.

A fig. 18 refere-se ao número de espécies obtido em cada ano. As curvas são bastante semelhantes, com média de $31 \pm 6$ espécies em 1992 e $29 \pm 9$ em 1993. O acúmulo total foi de 61 espécies em 1992 e 68 em 1993. 


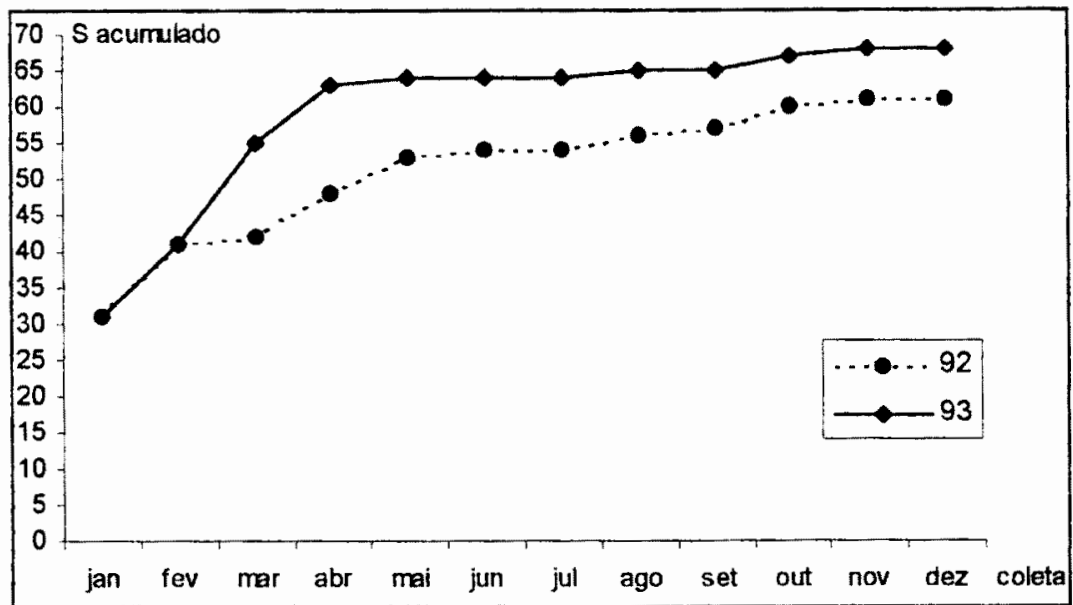

Fig.18 Número acumulado de espécies coletadas em armadilha tipo Shannon em área de cultivo irrigado de arroz (Fazenda Experimental do IAC - Pariquera-Açu, SP) levando-se em consideração cada ano de captura.

Já a fig. 19 mostra que o número acumulado de indivíduos divergiu nitidamente. A separação das curvas deveu-se à coleta de março de 1993, que representou $45 \%$ do total de indivíduos naquele ano (22243 de 48592), ao passo que, ao longo de 1992, obteve-se o total de 18524 indivíduos. Isso também provocou uma grande diferença no valor das médias - $1543 \pm 1042$ em 1992 e $4049 \pm 5910$ em 1993.

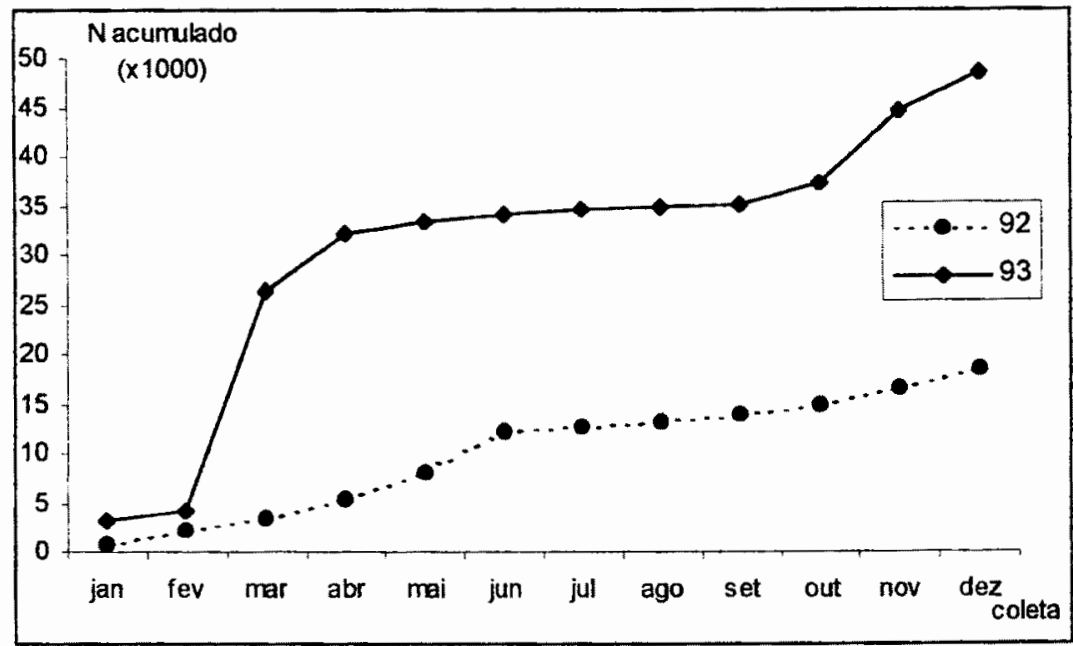

Fig. 19: Número acumulado de indivíduos coletados em armadilha tipo Shannon em área de cultivo irrigado de arroz (Fazenda Experimental do IAC - Pariquera-Açu, SP) levando-se em consideração cada ano de captura. 
Levando-se em consideração o período de janeiro de 1992 a dezembro de 1993, observa-se na fig.20 que a curva se estabilizou em 72 espécies (média $30 \pm 7$ ), sendo que de maio a dezembro de 1993 foi acrescentada apenas 1 espécie, em outubro.

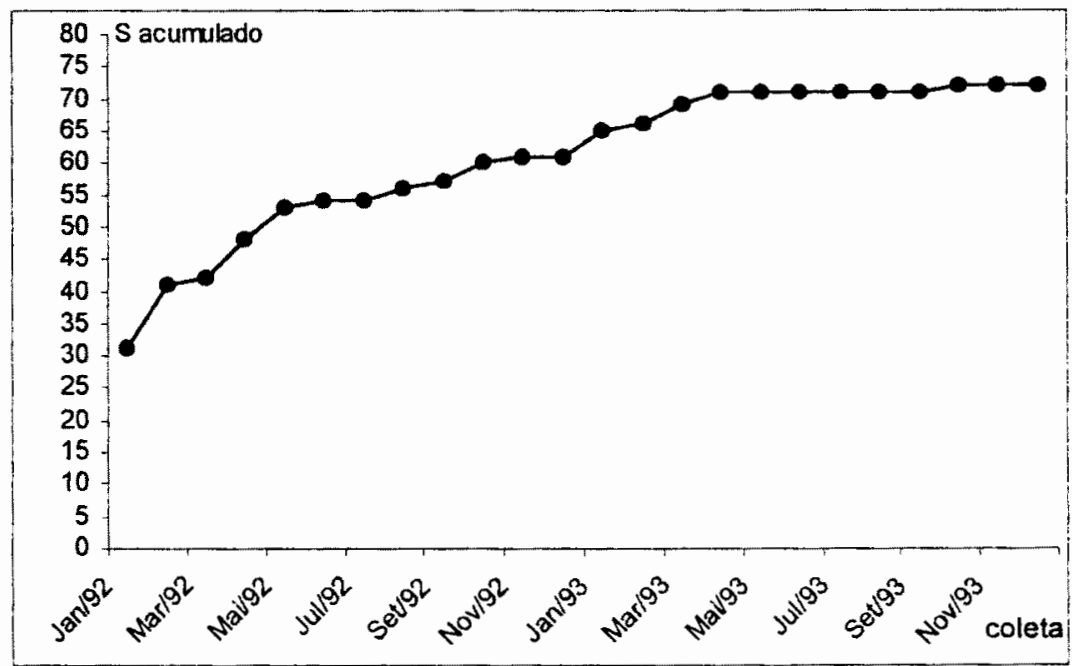

Fig.20: Número acumulado de espécies coletadas em armadilha tipo Shannon em área de cultivo irrigado de arroz (Fazenda Experimental do IAC - Pariquera-Açu, SP) durante o periodo de janeiro de 1992 a dezembro de 1993.

Em relação do número de indivíduos acumulado nos dois anos de captura, a fig.21, a seguir, destaca novamente o mês de março de 1993, responsável pelo desvio acentuado da curva, cujo valor máximo corresponde a 67116 mosquitos ( $\mathrm{N}$ médio $2796 \pm 4424$ ). 


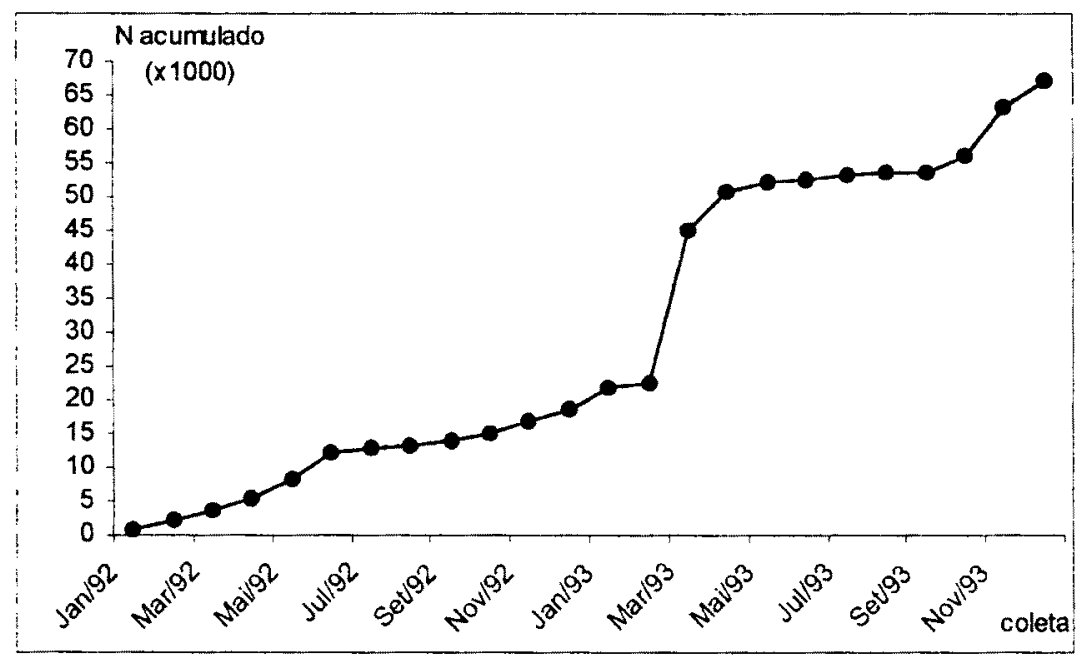

Fig.21: Número acumulado de individuos coletados em armadilha tipo Shannon em área de cultivo irrigado de arroz (Fazenda Experimental do IAC - Pariquera-Açu, SP) durante o período de janeiro de 1992 a dezembro de 1993.

\section{Diversidade}

As figuras que seguem ilustram o padrão de equidade, isto é, de distribuição dos valores de $\mathrm{N}$ entre os de $\mathrm{S}$, considerando os períodos totais de coleta em cada ambiente estudado.

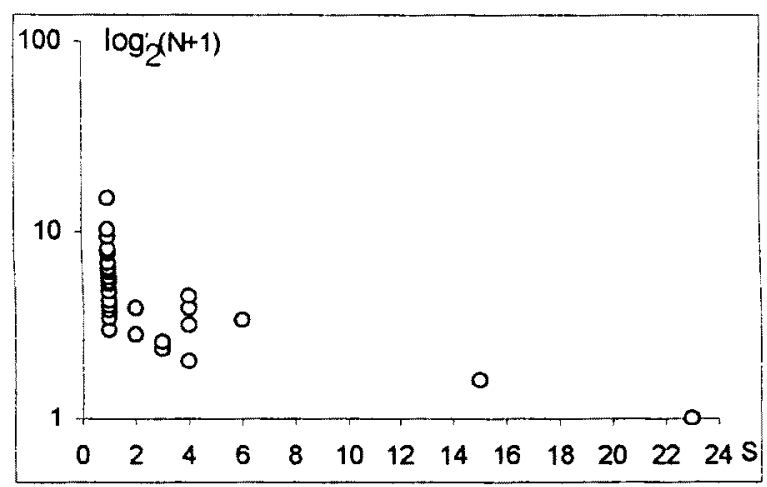

Fig.22: Distribuição das frequências (transformadas em $\log _{2}(\mathrm{~N}+1)$ ) e número de espécies em ambiente de mata primária ("Fonte" - Cananéia, SP), de fevereiro de 1992 a janeiro de 1993. 


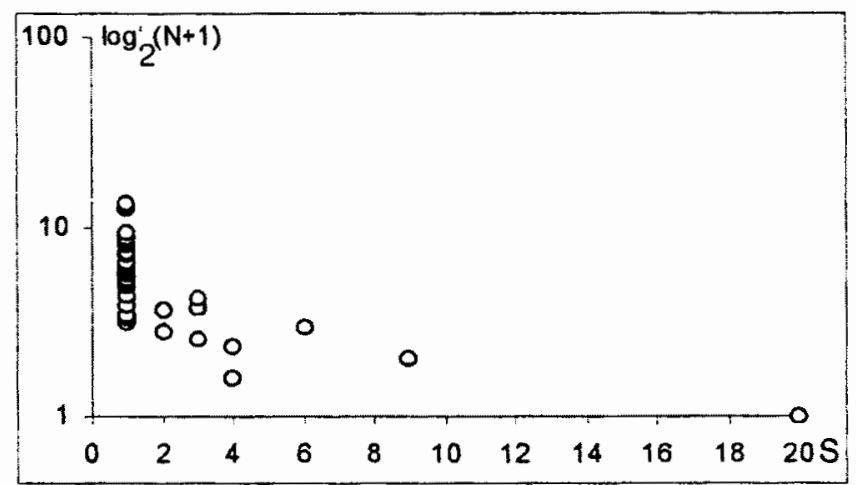

Fig.23: Distribuição das frequências $\left(\log _{2}(\mathrm{~N}+1)\right)$ e número de espécies em ambiente de mata primária (Síto Gentil - Cananéia, SP), de junho de 1993 a abril de 1995.

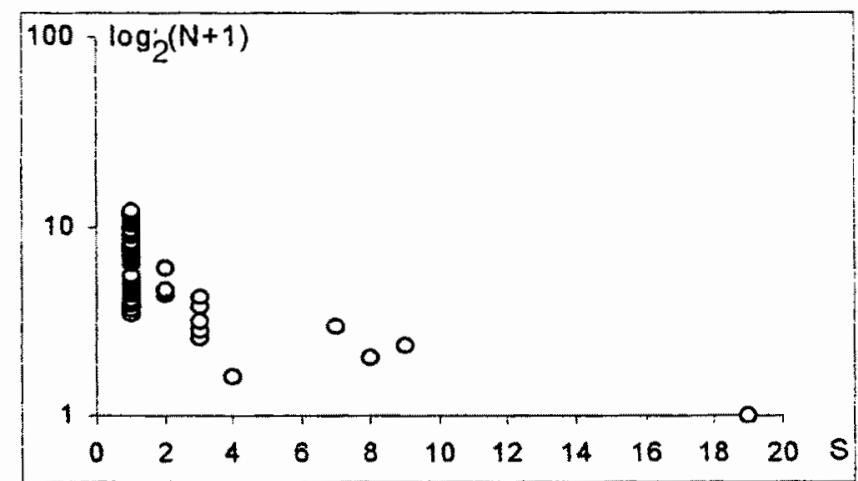

Fig.24: Distribuição das frequências $\left(\log _{2}(N+1)\right)$ e número de espécies em ambiente de mata residual (Mata A - Pariquera-Açu, SP), de janeiro de 1992 a dezembro de 1994.

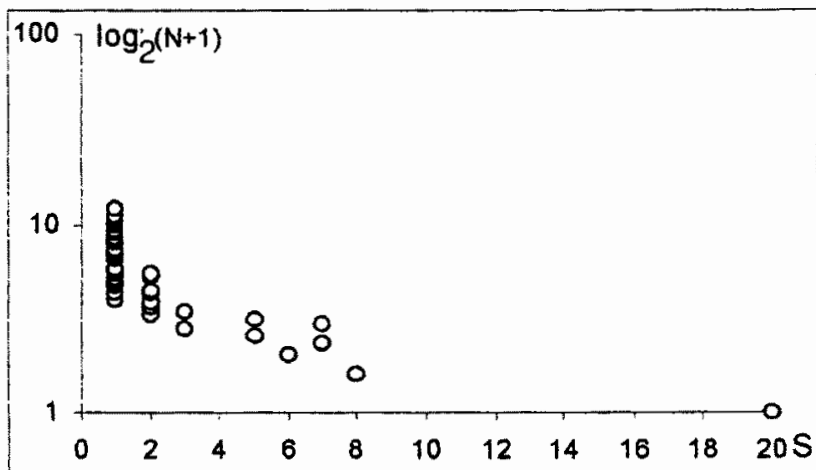

Fig.25: Distribuição das frequências $\left(\log _{2}(N+1)\right)$ e número de espécies em ambiente de mata residual (Mata B - Pariquera-Açu, SP), de fevereiro de 1992 a dezembro de 1994. 


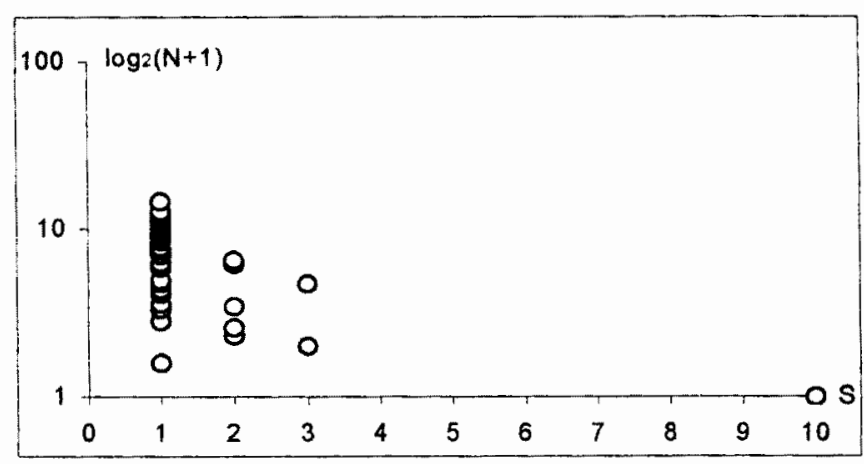

Fig.26: Distribuição das frequências $\left(\log _{2}(N+1)\right)$ e número de espécies em área de cultivo irrigado de arroz (Pariquera-Açu, SP), de janeiro de 1992 a dezembro de 1993 .

As figuras ilustram bem a maior proporção de espécies com apenas um indivíduo em todos os casos. Em outras palavras, pode-se dizer que a grande maioria dos $\mathrm{N}$ corresponde a uma espécie.

$\mathrm{Na}$ página seguinte, a tab.1 apresenta os índices de Margalef (MAGURRAN 1988; SERVICE 1993; HAYEK e BUZAS 1997) e Margalef padronizado, segundo HAYEK e BUZAS (1997). A tabela traz também o valor de S e $\mathrm{N}$ como referência. 
Tab. 1: Número de espécies (S) e indivíduos $(N)$ e valores dos índices de Margalef (d)e Margalef padronizado (d/dmax) calculados em cada tipo de ambiente: mata primária FT="Fonte" e $\mathrm{SG}=$ Sítio Gentil (Cananéia, SP), mata residual - $M A=$ mata $A$ e $M B=$ mata $B$ (Pariquera- $A c ̧ u, S P$ ) e $A R=$ arrozal (Pariquera-Açu, SP), para os respectivos períodos de coleta.

\begin{tabular}{|c|c|c|c|c|c|c|}
\hline \multicolumn{2}{|c|}{ Período Índices } & FT & $\mathrm{SG}$ & $\mathrm{MA}$ & $\mathrm{MB}$ & $\mathrm{AR}$ \\
\hline \multirow[t]{4}{*}{92} & $\mathrm{~S}$ & 94 & & 79 & 69 & 61 \\
\hline & $\mathrm{N}$ & 37383 & & 12023 & 4143 & 18524 \\
\hline & $\mathrm{d}$ & 8,833 & & 8,303 & 8,164 & 6,106 \\
\hline & $\mathrm{d} / \mathrm{dmax}$ & 0,0025 & & 0,0065 & 0,0164 & 0,0032 \\
\hline \multirow[t]{4}{*}{93} & $\mathrm{~S}$ & & 43 & 106 & 79 & 68 \\
\hline & $\mathrm{N}$ & & 7530 & 11817 & 10207 & 48592 \\
\hline & $\mathrm{d}$ & & 4,705 & 11,197 & 8,450 & 6,209 \\
\hline & $\mathrm{d} / \mathrm{dmax}$ & & 0,0056 & 0,0089 & 0,0076 & 0,0014 \\
\hline \multirow[t]{4}{*}{94} & $\mathrm{~S}$ & & 71 & 77 & 81 & \\
\hline & $\mathrm{N}$ & & 10860 & 5075 & 4683 & \\
\hline & d & & 7,533 & 8,907 & 9,465 & \\
\hline & $\mathrm{d} / \mathrm{dmax}$ & & 0,0064 & 0,0150 & 0,0171 & \\
\hline \multirow[t]{4}{*}{95} & $\mathrm{~S}$ & & 59 & & & \\
\hline & $\mathrm{N}$ & & 8200 & & & \\
\hline & $\mathrm{d}$ & & 6,436 & & & \\
\hline & $\mathrm{d} / \mathrm{dmax}$ & & 0,0071 & & & \\
\hline \multirow[t]{4}{*}{ Total } & $\mathrm{S}$ & 94 & 86 & 120 & 113 & 72 \\
\hline & $\mathrm{N}$ & 37383 & 26590 & 28915 & 19033 & 67116 \\
\hline & $\mathrm{d}$ & 8,833 & 8,343 & 11,585 & 11,366 & 6,388 \\
\hline & $\mathrm{d} / \mathrm{dmax}$ & 0,0025 & 0,00312 & 0,0041 & 0,0059 & 0,0010 \\
\hline
\end{tabular}

Na tab.2 estão os valores de $\alpha$ de Fisher (DAJOZ 1973; MAGURRAN 1988; SERVICE 1993; HAYEK e BUZAS 1997), com as respectivas variâncias e os intervalos de confiança.

Os valores dos índices de Margalef, Margalef padronizado e $\alpha$ de Fisher comportaram-se de modo semelhante, porém, não apresentaram correspondência com os valores de $\mathrm{S}$ ou $\mathrm{N}$ isoladamente (maior $\mathrm{S}$, maiores índices, por exemplo). 
Os valores obtidos para os pontos de mata residual (mata $A$ e B) foram, em geral, semelhantes entre si, bem como de um ano para o outro em cada um deles, e diferentes em relação à mata primária e arrozal. Além disso, os pontos de mata primária apresentaram diferenças entre si. No Sítio Gentil, inclusive, de um ano para 0 outro. Convém lembrar mais uma vez que neste local o número de coletas foi diferente a cada ano (7 em 1993, 12 em 1994 e 4 em 1995).

Tab.2: Valores do índice de Fisher $(\alpha)$, variância e intervalo de confiança de $95 \%$

(I.C.) em cada tipo de ambiente: mata primária - FT="Fonte" e SG=Sítio Gentil (Cananéia, SP), mata residual - MA=mata $A$ e $M B=$ mata $B$ (Pariquera-Açu, SP) e AR=arrozal (Pariquera-Açu, SP), para os respectivos periodos de coleta.

\begin{tabular}{|c|c|c|c|c|c|}
\hline Período & Local & $\alpha$ & $\begin{array}{l}\text { Variância } \\
(\alpha)^{\mathrm{a}}\end{array}$ & $\begin{array}{l}\text { Variância } \\
(\alpha)^{b}\end{array}$ & I.C. $(\alpha)^{c}$ \\
\hline \multirow[t]{4}{*}{92} & FT & 11,359 & 1,402 & 0,1498 & $10,223-12,495$ \\
\hline & MA & 11,004 & 1,572 & 0,199 & $9,903 \mid-12,104$ \\
\hline & $\mathrm{MB}$ & 11,760 & 2,004 & 0,341 & $10,584-12,936$ \\
\hline & AR & 7,854 & 1,011 & 0,119 & $7,069|-| 8,640$ \\
\hline \multirow[t]{4}{*}{93} & SG & 6,030 & 0,845 & 0,111 & $5,427 \mid-6,633$ \\
\hline & MA & 16,054 & 2,431 & 0,353 & $14,448|-| 17,659$ \\
\hline & MB & 11,486 & 1,691 & 0,230 & $10,337-12,635$ \\
\hline & AR & 7,778 & 0,890 & 0,090 & $7,000|-| 8,556$ \\
\hline \multirow[t]{3}{*}{94} & SG & 10,182 & 1,460 & 0,197 & $9,164|-| 11,200$ \\
\hline & MA & 12,878 & 2,154 & 0,358 & $11,590 \mid-14,166$ \\
\hline & MB & 13,913 & 2,390 & 0,412 & $12,522-15,304$ \\
\hline 95 & SG & 8,599 & 1,253 & 0,173 & $7,739|-| 9,459$ \\
\hline \multirow[t]{5}{*}{ Total } & FT & 11,359 & 1,402 & 0,1498 & $10,223|-| 12,495$ \\
\hline & SG & 11,043 & 1,418 & 0,166 & $9,939 \mid-12,147$ \\
\hline & MA & 15,693 & 2,087 & 0,246 & $14,124|-| 17,262$ \\
\hline & $\mathrm{MB}$ & 15,784 & 2,224 & 0,288 & $14,206-17,362$ \\
\hline & AR & 7,967 & 0,881 & 0,085 & $7,170\rfloor-8,764$ \\
\hline
\end{tabular}

s segundo MAGURRAN 1988; SERVICE 1993

b segundo HAYEK e BUZAS 1997

'segundo SERVICE 1993 


\section{Dominância}

O índice de Berger-Parker, que é um indicador de dominância comportou-se independentemente dos demais índices, bem como de $\mathrm{S}$ e $\mathrm{N}$, tanto em comparações entre os tipos de ambiente quanto em comparações entre os anos de captura.

Tab.3: Indicação das espécies dominantes em cada local, segundo o índice de Berger-Parker.

\begin{tabular}{|c|c|c|}
\hline Coletas & LOCAL/Espécie & Berger-Parker \\
\hline & FONTE* & \\
\hline \multirow[t]{2}{*}{ total } & Anopheles cruzii & 0,890 \\
\hline & SÍTIO GENTIL* & \\
\hline 93 & Anopheles crusii & 0,346 \\
\hline 94 & Coquillettidia chrysonotum/albifera & 0,296 \\
\hline 95 & Anopheles cruzii & 0,494 \\
\hline \multirow[t]{2}{*}{ total } & Anopheles crusii & 0,364 \\
\hline & MATA A** & \\
\hline 92 & Culex nigripalpus & 0,182 \\
\hline 93 & Aedes serratus & 0,174 \\
\hline 94 & Coquillettidia chrysonotum/albifera & 0,176 \\
\hline \multirow[t]{2}{*}{ total } & Aedes serratus & 0,139 \\
\hline & MATA B** & \\
\hline 92 & Aedes serratus & 0,224 \\
\hline 93 & Aedes serratus & 0,246 \\
\hline 94 & Psorophora albigenu & 0,131 \\
\hline \multirow[t]{2}{*}{ total } & Aedes serratus & 0,209 \\
\hline & ARROZAL & \\
\hline 92 & Aedes scapularis & 0,134 \\
\hline 93 & Anopheles albitarsis espécie B & 0,443 \\
\hline Total & Anopheles albitarsis espécie B & 0,348 \\
\hline
\end{tabular}

A identificação de An. albitarsis (espécie B) baseia-se em FORATTINI e colaboradores (1995a).

A seguir, serão apresentadas figuras que ilustram a distribuição das frequências mensais da espécie dominante, segundo o índice de Berger-Parker, e dos valores mensais de precipitação em cada ambiente, para os periodos totais de coleta. 
Na mata primária, a possível correlação entre frequência mensal de An. cruzii e precipitação média mensal dos meses correspondentes foi estimada pelo coeficiente de Pearson ( $r$ ), cujos valores foram $-0,24$ na Fonte e 0,45 no Sítio Gentil. O $r$ calculado para frequência mensal da espécie e precipitação média do mês anterior foi 0,55 na Fonte e 0,25 no Sítio Gentil.

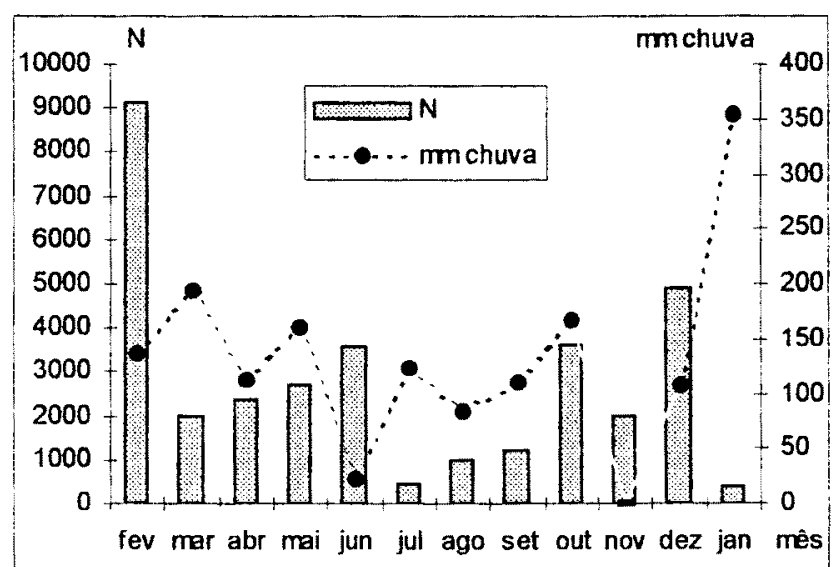

Fig.27: Distribuição das frequências mensais de An.cruzii e valores de precipitação $(\mathrm{mm})$ em área de mata primária ("Fonte" - Cananéia, SP). Coletas em armadilha tipo Shannon, de fevereiro de 1992 a janeiro de 1993.

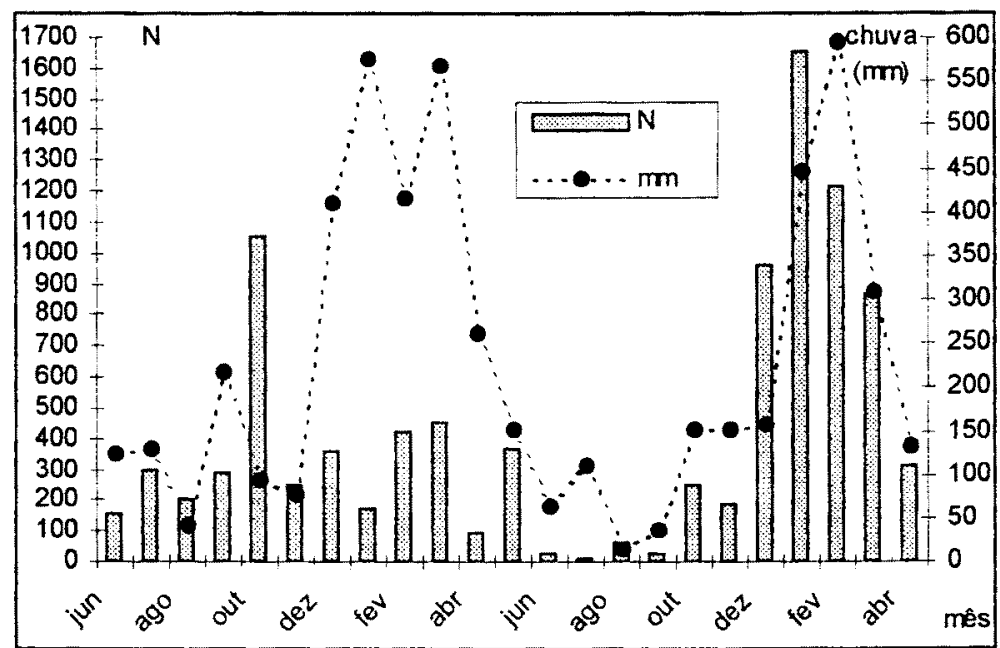

Fig.28: Distribuição das frequências mensais de An.cruzii e valores de precipitação $(\mathrm{mm})$ em área de mata primária (Sítio Gentil Cananéia, SP). Coletas em armadilha tipo Shannon, de junho de 1993 a abril de 1995. 
Nas áreas de mata residual, os valores de $r$ para frequência mensal de Ae. serratus e chuva foram 0,5 na mata A e 0,09 na mata B. Para a frequência mensal desta espécie e chuva no mês anterior or foi 0,42 na mata $A$ e 0,01 na mata $B$.

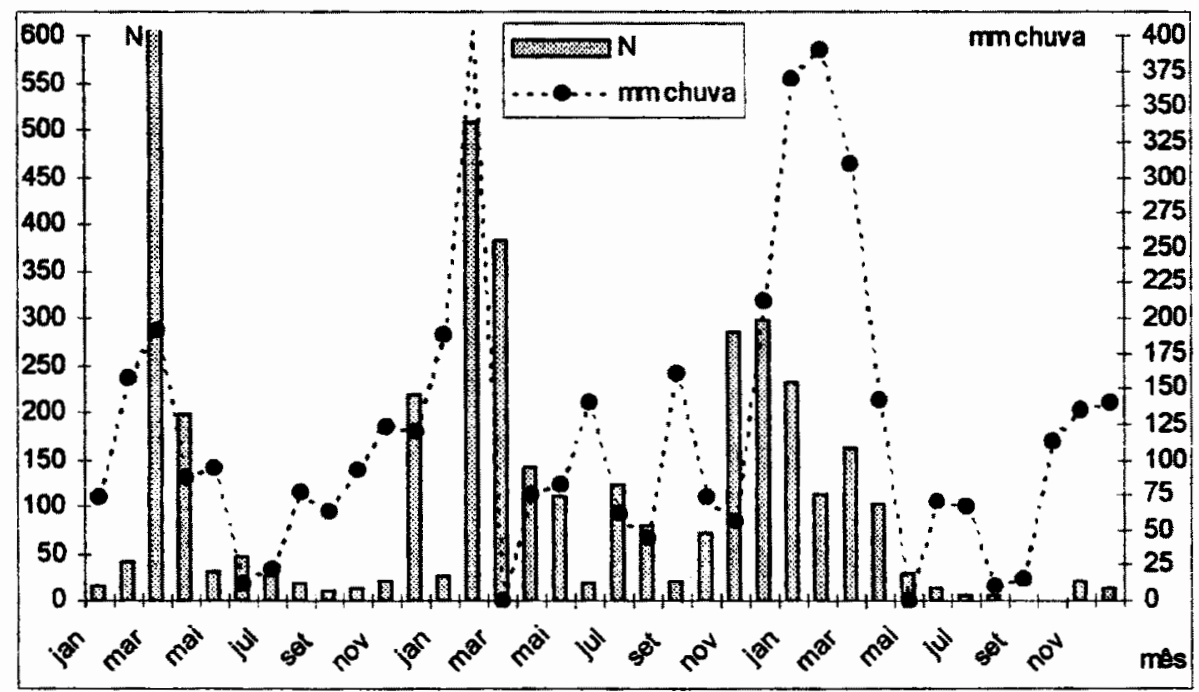

Fig.29: Distribuição das frequências mensais de Ae.serratus e valores de precipitação $(\mathrm{mm})$ em área de mata residual (mata A - Fazenda Experimental - Pariquera-Açu, SP). Coletas em armadilha tipo Shannon, de janeiro de 1992 a dezembro de 1994.

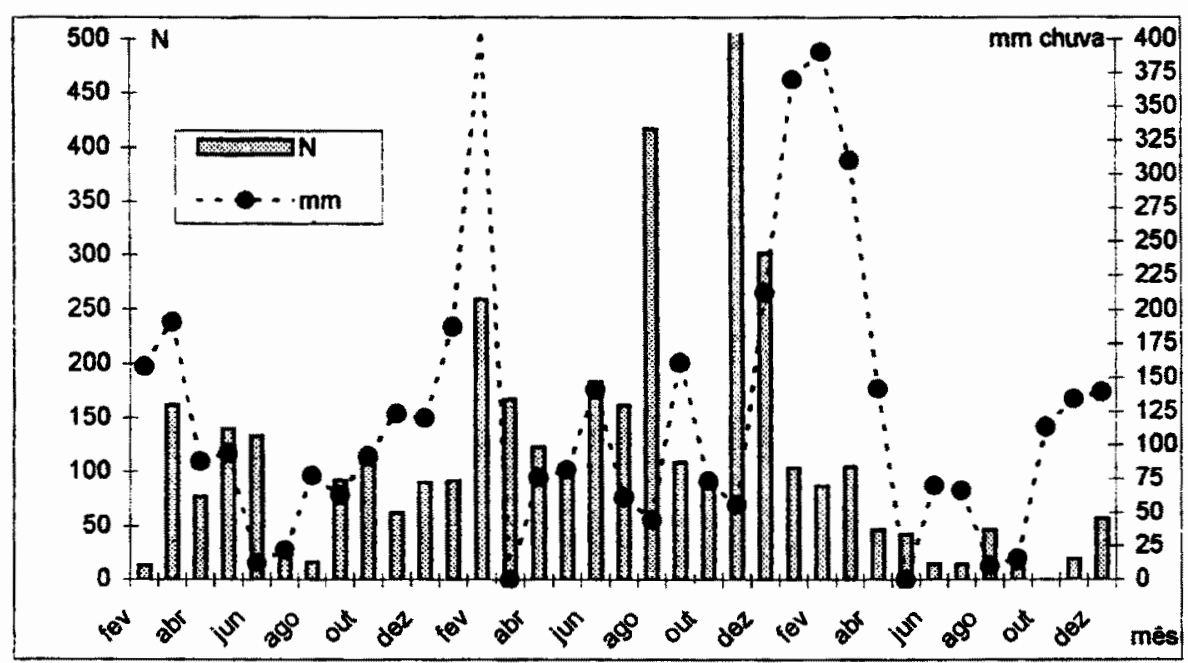

Fig.30: Distribuição das frequências mensais de Aeserratus e valores de precipitação $(\mathrm{mm})$ em área de mata residual (mata B - Fazenda Experimental - Pariquera-Açu, SP). Coletas em armadilha tipo Shannon, de fevereiro de 1992 a dezembro de 1994. 


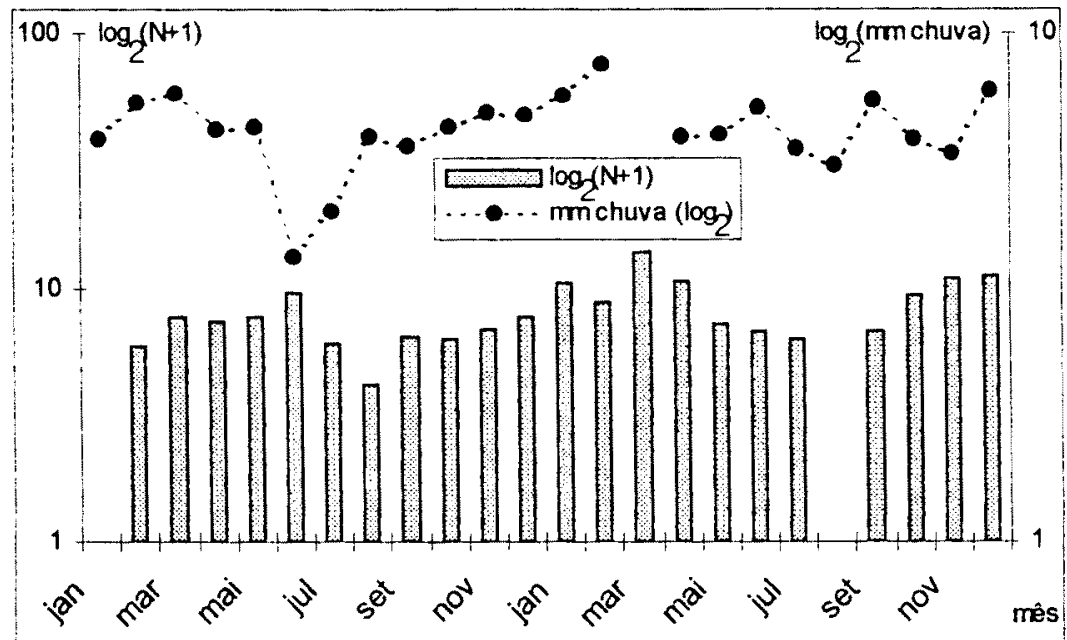

Fig. 31: Distribuição das frequências mensais de An.albitarsis (espécie B) e valores de precipitação ( $\mathrm{mm}$ ) em área de cultivo irrigado de arroz (Fazenda Experimental do IAC - Pariquera-Açu, SP). Coletas em armadilha tipo Shannon, de janeiro de 1992 a dezembro de 1993

Em relação à área de cultivo irrigado de arroz (fig.31), a coleta de março de 1993, com 13249 indivíduos, apresentou forte contraste com os demais meses de captura, cujos valores de $\mathrm{N}$ variaram entre zero e 2015 . Por essa razão, os dados foram transformados em logaritmo $\left(\log _{2}(N+1)\right)$.

$\mathrm{O}$ coeficiente de Pearson para frequência mensal de An. albitarsis (espécie $B$ ) e chuva foi 0,14 e para a frequência desta espécie e chuva no mês anterior, 0,78 . 


\section{Constância}

Apresentam-se aqui os resultados de constância (DAJOZ 1973) das espécies. As figuras 32 a 36 ilustram as proporções relativas entre espécies constantes ( $x$, presentes em mais de 50\% das coletas), acessórias (y, presentes em 25 a $50 \%$ das coletas) e acidentais ( $z$, presentes em menos de $25 \%$ das coletas) em cada tipo de ambiente, considerados os periodos totais de coleta.

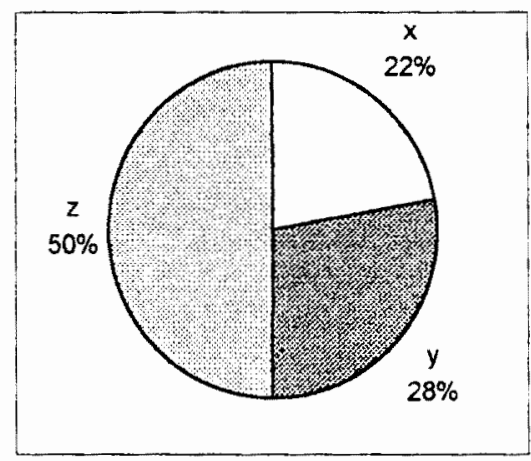

Fig.32: Porcentagem de espécies constantes $(\mathrm{x})$, acessórias (y) e acidentais( $\mathrm{z}$ ) em ambiente de mata primária ("Fonte" - Cananéia, SP).

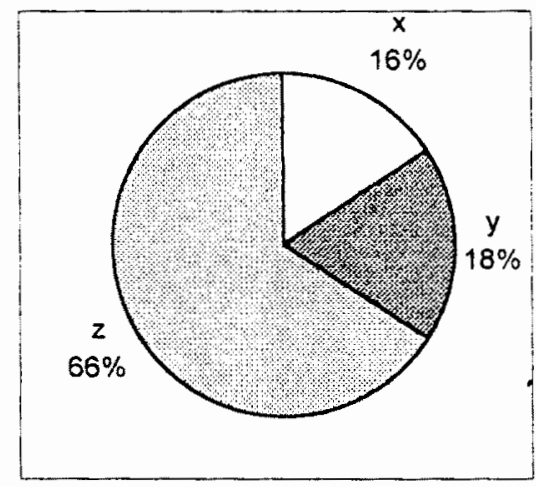

Fig.34: Porcentagem de espécies constantes $(\mathrm{x})$, acessórias (y) e acidentais $(\mathrm{z})$ em ambiente de mata residual ("mata A" - Fazenda Experimental do IAC Pariquera-Açu, SP)

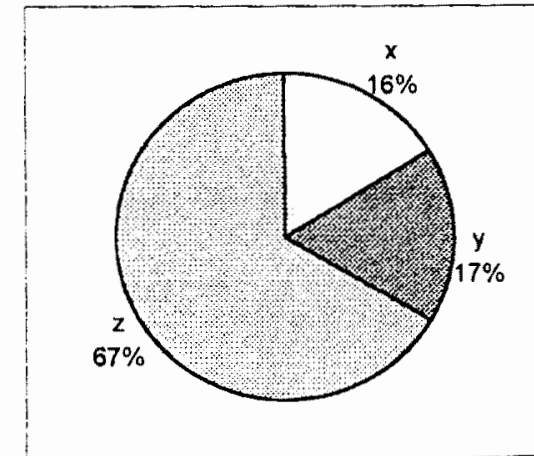

Fig.33: Porcentagem de espécies constantes $(\mathrm{x})$, acessórias $(\mathrm{y})$ e acidentais $(z)$ em ambiente de mata primária (Sítio Gentil - Cananéia, SP).

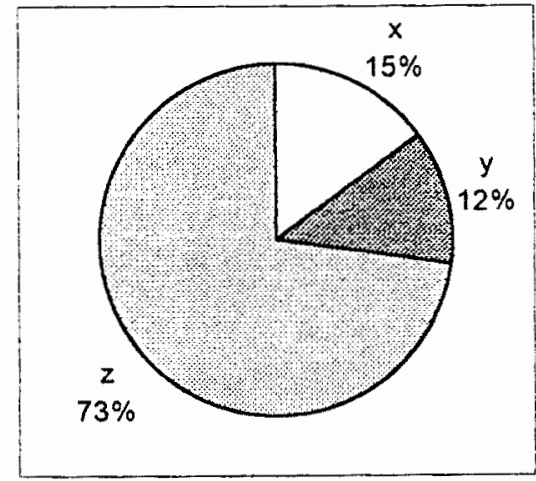

Fig.35: Porcentagem de espécies constantes $(x)$, acessórias (y) e acidentais( $z$ em ambiente de mata residual ("mata B" - Fazenda Experimental do IAC Pariquera-Açu, SP). 


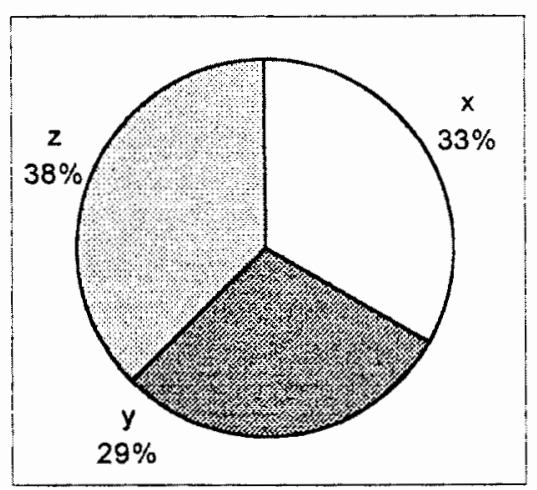

Fig.36: Porcentagem de espécies constantes $(\mathrm{x})$, acessórias (y) e acidentais ( $\mathrm{z}$ ) em área de cultivo irrigado de arroz (Fazenda Experimental do IAC Pariquera-Açu, SP)

Em todos os ambientes estudados, as espécies acidentais representam a maior porcentagem e as proporções de espécies constantes e acessórias foram semelhantes tanto entre si, quanto entre os ambientes de mata. $\mathrm{Na}$ "Fonte", porém, as espécies acidentais possuem proporção menor do que nos demais ambientes de mata. A área de cultivo irrigado de arroz também apresentou padrão diferente, com proporções quase equivalentes entre as três categorias.

$\mathrm{Na}$ página seguinte, a tab. 4 indica quais foram as espécies constantes em pelo menos um dos ambientes estudados. A tabela completa, que inclui também as espécies acessórias e acidentais encontra-se no apêndice (tab.28). 
Tab.4: Espécies constantes (presentes em mais de 50\% das coletas) em pelo menos um dos ambientes de estudo - mata primária (FT, SG - Cananeia, SP), mata residual (MA, MB PariqueraAçu, SP) e área de cultivo irrigado de arroz (AR - PariqueraAçu, SP), considerados os respectivos periodos de coletas em armadilha tipo Shannon (FT - 12, SG - 23, MA - 36, MB $35, \mathrm{AR}-24$ meses)

\begin{tabular}{|c|c|c|c|c|c|}
\hline Espécie & FT & SG & MA & MB & AR \\
\hline Ae.grupoSematus(serratus/nubilus) & $x$ & $x$ & $x$ & $x$ & $x$ \\
\hline Ae.prox.perventor ou hortator & $x$ & $z$ & & $z$ & \\
\hline Ae.scapularis & $x$ & $x$ & $x$ & $x$ & $x$ \\
\hline Ae.serratus & $x$ & $y$ & $x$ & $x$ & $x$ \\
\hline An.albitarsis esp. A & & & $z$ & & $x$ \\
\hline An.albitarsis esp. $B$ & & $z$ & $z$ & & $x$ \\
\hline An.albitarsis & & & $z$ & & $x$ \\
\hline An.bellator & $x$ & $\mathrm{x}$ & & $z$ & \\
\hline An.cruzii & $x$ & $x$ & $y$ & $x$ & y \\
\hline An.evansae & $z$ & & $z$ & $z$ & $x$ \\
\hline An.intermedius & $x$ & $z$ & $y$ & $z$ & $x$ \\
\hline An.oswaldoi & $z$ & & z & $z$ & $x$ \\
\hline An.prox.mediopunctatus & $x$ & $z$ & $x$ & $x$ & $z$ \\
\hline An.strodei & $z$ & & $z$ & & $x$ \\
\hline Cq.chnsonotum ou albifera & $x$ & $x$ & $x$ & $x$ & $x$ \\
\hline Cq.hermanoi & & $z$ & $x$ & $z$ & $x$ \\
\hline Cq.juxtamansonia & $z$ & y & $x$ & $z$ & $x$ \\
\hline Cq.venezuelensis & $y$ & $x$ & $x$ & $x$ & $x$ \\
\hline Cx.(Cux.) grupo Coronator & $x$ & $x$ & $x$ & $y$ & $x$ \\
\hline Cx.(Cux.)nigripalpus & $x$ & y & $x$ & $x$ & $x$ \\
\hline Cx.(Cux.)sp. & $x$ & $x$ & $x$ & $x$ & $x$ \\
\hline Cx.(Mel.)grupo Atratus & $x$ & $z$ & $y$ & $x$ & $z$ \\
\hline Cx.(Mel.)ocossa & & $z$ & $y$ & $z$ & $x$ \\
\hline Cx.(Mel.)nibeirensis & $x$ & $x$ & $x$ & $x$ & $x$ \\
\hline CX.(Mel.)sacchettae & $x$ & $x$ & $x$ & $x$ & $x$ \\
\hline Li.durhami & $y$ & $x$ & $x$ & $x$ & $z$ \\
\hline Liffavisetosus & $z$ & $y$ & $x$ & $x$ & \\
\hline Ma.(Man.)humeralis & & & $z$ & & $x$ \\
\hline Ma.(Man.)indubitans & & $z$ & $x$ & $x$ & $x$ \\
\hline Ma.(Man.)sp & $z$ & $y$ & $x$ & $y$ & $z$ \\
\hline Ph.fiabellata & $x$ & $z$ & & & \\
\hline Ph.palmata/diabolica & $x$ & $y$ & $z$ & $z$ & \\
\hline Ps.ciliata & & & & & $x$ \\
\hline Ps.discrucians & & & $z$ & $z$ & $x$ \\
\hline Ps.ferox & $x$ & $y$ & $x$ & $x$ & $z$ \\
\hline Ru.reversa & $x$ & $y$ & $y$ & $y$ & \\
\hline Ur.geometrica & $z$ & $x$ & $z$ & $z$ & $y$ \\
\hline Wy.contusa & $x$ & $x$ & $x$ & $x$ & \\
\hline Wy.mystes ou finlayi & $x$ & $z$ & $z$ & $z$ & \\
\hline total de espécies constantes & 21 & 13 & 19 & 17 & 24 \\
\hline
\end{tabular}




\section{Similaridade}

A tabela a seguir apresenta os valores do índice de Sorensen, que traduz o grau de associação ou similaridade de ambientes (ou amostras), dois a dois. $O$ valor máximo que esse índice pode assumir é 1 , o que significa que os ambientes ou amostras possuem faunas idênticas. O valor mínimo é zero e significa que os dois ambientes não apresentam espécies em comum.

Tab.5: Índice de Sorensen para os ambientes, comparados 2 a 2 . Valores referentes aos periodos totais de coleta: Fonte - 12, Sítio Gentil - 23, mata $A-36$, mata $B-35$, arrozal -24 meses.

\begin{tabular}{lcccc}
\hline & \multicolumn{2}{c}{ Mata Primária* } & \multicolumn{2}{c}{ Mata Residual** } \\
\cline { 2 - 5 } & Fonte & Sitio Gentil & Mata A & Mata B \\
\hline Sítio Gentil & 0,708 & & & \\
Mata A & 0,636 & 0,699 & & \\
Mata B & 0,705 & 0,744 & 0,858 & \\
Arrozal** & 0,41 & 0,519 & 0,677 & 0,573 \\
\hline
\end{tabular}

*Cananéia, SP; **Pariquera-Açu, SP

\section{Espécies de Importância Médica}

A indicação de espécies de interesse médico baseia-se em FORATTINI (1962. 1965a, 1965b), BERGE (1975) e CONSOLI e LOURENÇODE-OLIVEIRA (1994)

\subsection{Gênero Anopheles}

An. albitarsis espécie A-mata residual (mata A) e arrozal

An. albitarsis espécie B - mata primária (Sítio Gentil), residual (mata A) e arrozal

An. albitarsis - mata residual (mata A) e arrozal

An. belator - mata primária ("Fonte" e Sítio Gentil) e mata residual (mata B)

An. cruzii-todos os ambientes

An. evansae - todos os ambientes, exceto Sítio Gentil 
An. galvaoi - mata primária ("Fonte"), residual (mata A) e arrozal An. oswaldo - todos os ambientes, exceto Sítio Gentil An. strodei - mata primária ("Fonte"), residual (mata A) e arrozal An. triannuiatus - mata residual (mata A e B) e arrozal

\subsection{Gênero Aedes}

Ae. albifasciatus - arrozal

Ae. albopictus - mata residual (matas A e B)

Ae. fluviatilis - mata residual (mata A)

Ae. fulvus - todos os ambientes, exceto Sítio Gentil (mata primária)

Aedes grupo Serratus (hastatus/oligopistus) - todos os ambientes, exceto arrozal Ae. hastatus - mata primária ("Fonte" e Sítio Gentil)

Ae. oligopistus - mata primária ("Fonte" e Sítio Gentil) e mata residual (mata B)

Ae. scapularis - todos os ambientes

Ae. serratus - todos os ambientes

\subsection{Gênero Culex}

Cx. corniger - mata residual (mata A) e arrozal

Cx. coronator - mata residual (mata A)

$C x$. declarator - mata primária ("Fonte") e mata residual (mata A e B)

Cx. nigripalpus - todos os ambientes

Cx. caudelli - mata residual (mata B)

Cx. delpontei - mata residual (mata A e B) e arrozal

Cx. dunni-mata primária (Sítio Gentil) e residual (mata A e B)

Cx. ribeirensis - todos os ambientes

$C x$ sacchettae - todos os ambientes

Cx. spissipes - mata residual (mata A e B)

Cx. taeniopus - mata primária ("Fonte" e Sítio Gentil) e residual (mata A e B) 


\subsection{Outras Espécies}

Coquillettidia venezuelensis - todos os ambientes

Limatus durhami - todos os ambientes

Li. flavisetosus - todos os ambientes, exceto arrozal

Mansonia indubitans - todos os ambientes, exceto "Fonte"

Ma. titillans - todos os ambientes, exceto "Fonte"

Psorophora albipes - mata primária (Sítio Gentil) e mata residual (mata A e B)

Ps. ciliata - arrozal

Ps. ferox - todos os ambientes

Sabethes chloropterus - mata residual (mata A e B)

Trichoprosopon digitatum -- "Fonte" e mata residual (mata A e B)

Wyeomyia aporonoma - "Fonte" e mata residual (mata A e B) 


\section{DISCUSSÃO}

A idéia de que o número de espécies e de indivíduos aumenta conforme a área e/ou tempo de estudo é intuitiva. Porém, sabe-se que diversos fatores interferem nessas contagens. Um deles é a distribuição espacial dos indivíduos. Existem basicamente três padrões: uniforme, contagioso ou gregário e aleatório (DAJOZ 1973; ODUM 1988; BEGON e cols 1996). Outro fator que não pode deixar de ser mencionado são as flutuações populacionais, cujos determinantes podem ser dependentes ou independentes da densidade. Aqueles referem-se às relações intra e interespecíficas (predação, parasitismo, competição) e estes, aos fenômenos climáticos, físico-químicos e outros abióticos (DAJOZ 1973; BEGON e cols 1996). Os fatores são conhecidos, mas os mecanismos que os regem não estão completamente elucidados. Além disso, limitações operacionais impedem investigações em escala real de tempo e espaço. Esquemas amostrais e testes estatísticos procuram representar eventos da natureza.

DOBYNS (1997) comenta sobre o dilema do pesquisador, sobretudo quando se refere a ambientes complexos - esgotar a área mediante coletas repetidas no mesmo ponto ou "varrer" o maior número possível de microhabitats.

Semelhantemente, LANDAU e colaboradores (1999) avaliaram o desempenho de um número estipulado de capturas realizadas em curto prazo ou ao longo de meses. O esquema intensivo detectou $15 \%$ a menos de espécies que o extensivo, mas ocupou apenas a metade do tempo.

Portanto, o objetivo da pesquisa, limitações de tempo, capacidade operacional e financeira e, principalmente, o objeto de estudo determinam o protocolo da pesquisa.

As curvas de $\mathrm{S}$ e $\mathrm{N}$ acumulado apresentadas no capítulo anterior referem-se a diferentes períodos de captura, em número de coletas e época das mesmas. Isso limita seriamente comparações estatísticas mas, ainda que empiricamente, permitem visualizar os dados.

Em relação ao número de espécies, só são adicionadas aquelas que não estiveram presentes na(s) coleta(s) anterior(es). Assim sendo, uma curva que 
atingisse um patamar, teoricamente, indicaria que todas as espécies que ocorrem no local foram coletadas.

Nas primeiras coletas, espera-se que a curva ascenda de forma mais acentuada e, à medida em que o número de coletas fosse aumentando, o número adicionado de espécies iria diminuindo até que a curva se estabilizasse. Obviamente deve-se ter em mente que o horário de captura, época do ano, condições do dia, tipo de armadilha, de isca entre outras variáveis, selecionaram as espécies capturadas.

Em se tratando de mosquitos, aceita-se que estes são oportunistas quanto à hematofagia quando o homem invade seu habitat. Desse modo, espécies diurnas, acrodendrófilas e/ou com preferência por outros animais, que não o homem, para realizar hematofagia podem não ter ocorrido nas coletas ou terem sido subestimadas.

Posto isto, as curvas de $\mathrm{S}$ acumulado, de fato, adicionaram mais espécies nas primeiras capturas em todos os ambientes para todos os períodos. Porém, com exceção da mata A (residual) em 1993 e do arrozal, as curvas não se estabilizaram quando foram considerados períodos de até um ano. Mesmo considerando-se os períodos totais de captura, apenas a mata A (residual) e arrozal atingiram patamar. Este, inclusive, de valor maior do que aquele das curvas parciais. Os valores de $\mathrm{S}$ para os periodos totais variaram de local para local, mas nos pontos de mata residual foram maiores (mata A - 120 e mata B - 113), seguidos dos pontos de mata primária (Fonte -94 e Sítio Gentil - 86, a despeito do período de capturas ter sido maior nesta localidade do que naquela). Finalmente, na área de cultivo irrigado de arroz acumulou-se o total de 72 espécies.

Para os períodos de um ano, também as áreas de mata residual e primária atingiram maiores valores de $\mathrm{S}$ (média 84). No arrozal, em 1992 foram 61 espécies e em 1993, 68. Na mata B (residual) em 1992 foram realizadas capturas em 11 meses, totalizando 69 espécies. No Sítio Gentil (mata primária), o período de julho a dezembro de 1993 acumulou apenas 43 espécies, ao passo que de janeiro a abril de 1995 obteve-se 59 espécies.

Isso mostra que um ano de captura representa uma boa amostragem da riqueza de espécies do local, já que os acréscimos diminuem bastante após 5 ou 6 meses, mas mesmo ao final de 3 anos, verifica-se ainda a adição de espécies. 
Quando se compara diferentes anos no mesmo local, também surgem diferenças. Ainda que as curvas tenham se comportado de forma semelhante, nos ambientes de mata residual (A e B) um dos anos ficou destacado dos demais em função dos valores de $S$. $E$, mesmo quando os valores foram próximos ou até coincidentes, estes podem envolver diferentes composições de espécies.

O conceito mais amplo e intuitivo de biodiversidade confunde-se com a riqueza de espécies, isto é, o número de espécies do local. GASTON (1996) salienta vários problemas práticos referentes à medida de riqueza de espécies e que dificultam comparações. Entre eles a escala de tempo e espaço.

Estudos podem ser feitos em áreas diferentes em tamanho $\mathrm{e}$ características topográficas, com diferentes unidades e números de amostras. Ainda, estudos com objetivos semelhantes, mas conduzidos em áreas distantes entre si em escala latitudinal. Raciocínio semelhante pode ser feito com relação aó tempo; variações quanto ao horário, número de dias, estações do ano, antes e/ou após impactos ambientais ou mesmo intervalo grande de anos. Além disso, fatores biológicos: fora os já mencionados, taxa de colonização e extinção de espécies entre outros. Por exemplo, CODDINGTON e colaboradores (1991), citado por DOBYNS (1997, p.151), em estudos de diversidade de aranhas, propôs que uma amostra representativa para se estimar a riqueza de espécies desse táxon deve ser 10 vezes o número de espécies adultas presentes numa área previamente estudada. Embora a metodologia de captura e a bionomia desses animais seja muito diferente da de mosquitos, o estabelecimento de critérios desse tipo constitui-se como ferramenta valiosa de pesquisa.

Diferenças marcantes aparecem em trabalhos com metodologia semelhante para mesmo táxon: ZANUNCIO e colaboradores (1998) coletaram 459 e 300 espécies de Lepidoptera ao longo de 6 meses em duas localidades de Minas Gerais. BRAGANÇA e colaboradores (1998), comparando vários locais obtiveram, ao todo, 790 espécies, em 5 meses de levantamento no Espírito Santo (média de 335 espécies por localidade). SWENGEL (1998) em levantamentos de 5 a 7 anos (amostragens de 1 a 100 dias) em 27 pontos nos Estados Unidos (Missouri, Minesota e Wiscosin) capturou, no máximo, pouco mais de 50 espécies por local. LANDAU e colaboradores (1999), também nos Estados Unidos (Louisiana), comparando 
diferentes esquemas de captura, a longo e curto prazo, coletaram 314 e 261 espécies, respectivamente.

As dificuldades para se interpretar as curvas do número acumulado de individuos não são menores. Diferentemente das de espécies, não se espera que estas curvas se estabilizem. Os mesmos fatores que interferem no acúmulo de espécies, expostos anteriormente, cabem aqui. $\mathrm{O}$ número de indivíduos talvez reflita a intensidade dessas interferências.

Em relação aos períodos totais de captura, novamente a área de cultivo irrigado de arroz destacou-se das área de mata primária e residual. Os valores diferiram muito de um ano para outro e também no total, quando comparados com os demais ambientes. 1992 acumulou 18524 indivíduos e 1993, 48592 (total dos 2 anos 67116). Conforme já mencionado nos resultados, foi a coleta de março de 1993 que separou as curvas dos dois anos, embora as densidades de mosquitos nos outros meses tenham sido elevadas em relação ao ano de 1992 e aos demais locais, exceto se comparado com a Fonte (37383 mosquitos em 1 ano). Uma possivel explicação biológica seria crescimento populacional súbito, fenômeno que ocorre em várias espécies. A área de cultivo irrigado de arroz seria local propício para que isso ocorra, uma vez que há fase do ciclo desse vegetal durante a qual o terreno fica inundado, permitindo a formação de criadouro permanente.

Entre os ambientes de mata, as diferenças no número de indivíduos são mais marcantes do que no número de espécies. Se lembrarmos que na Fonte (mata primária) foram coletados 37383 mosquitos em somente 12 meses de captura, os contrastes aumentam. No outro ponto de mata primária (Sítio Gentil) 4 meses de captura em 1995 obtiveram maior rendimento ( 8200 mosquitos) do que 7 meses em $1993(7530)$.

$\mathrm{Na}$ matas residuais ( $\mathrm{A} \mathrm{e} \mathrm{B}$ ), tivemos 2 anos com curvas de valores bastante semelhantes entre si e um ano destacado, como ocorreu com as curvas de espécies. Porém, a curva destacada não corresponde à de espécies. Na mata $\mathrm{A}, \mathrm{o}$ ano de 1994 contribuiu com menos da metade (5075 indivíduos) do que os outros anos (1992 - 12023 e 1993 - 11817); os valores de espécies foram: $79 \mathrm{em} \mathrm{1992,} 106 \mathrm{em}$ 1993 e 77 em 1994. Na mata B verificou-se o inverso: o ano de 1993, que se 
destacou, acumulou mais do que o dobro do número de indivíduos (10207) dos demais - 4143 em 1992 e 4683 em 1994.

Essa grande variação do número de espécies e, maior ainda do número de individuos, é comum em diferentes combinações de tempo e espaço. Embora comum, tal fato continua sem explicação satisfatória. Diferenças quanto à metodologia, conforme já discutido, interferem bastante e podem justificar parcialmente as variações em função do número de capturadores, uso de aspirador ou tubo de ensaio, tipo de isca, horário, duração e local de captura (tipos de ambiente e estratificação vertical). Além disso, GASTON (1996) salienta a importância da taxonomia na contagem da riqueza de espécies e aponta dois tipos básicos de erro: classificar 1 espécie como 2 ou mais, e considerar 2 ou mais espécies como sendo 1.

RACHOU e colaboradores (1958), em capturas mensais com isca humana de 24 horas em Ponta Grossa (Santa Catarina) coletaram em área de mata 21361 mosquitos referentes a 25 espécies ao longo de 3 anos. Neste levantamento, 2 espécies apontadas pertencem atualmente a outro gênero.

FORATTINI e colaboradores (1968) em 6 meses de capturas noturnas, no solo e em copa arbórea, obtiveram 28329 mosquitos distribuidos entre 35 espécies no Nordeste do Estado de São Paulo. Também neste trabalho, Runchomyia aparece como subgênero de Trichoprosopon e, atualmente estes grupos constituem gêneros distintos.

TEODORO e colaboradores (1994) identificaram às margens de um açude em Querência do Norte (Paraná), com isca humana (4:00 8:00 e 16:00 20:00 h) durante um ano, 5923 mosquitos de 32 espécies. Em publicação posterior, TEODORO e colaboradores (1995), próximo ao lago de Itaipu (Paraná), a equipe identificou em um ano 41 espécies englobando 21280 mosquitos coletados em armadilha tipo Shannon e 1010 com isca humana.

Ao se considerar a riqueza de espécies em conjunto com suas respectivas abundâncias, fala-se em diversidade ou biodiversidade. Não é raro acontecer de localidades apresentarem o mesmo número de espécies e total de indivíduos. Daí a importância da equidade ("eveness") da comunidade, ou seja, como os individuos se distribuem entre as espécies (MAGURRAN 1988; BEGON e cols 1996; GASTON 1996; HAYEK e BUZAS, 1997). 
As figuras do item 2 do capitulo anterior ilustram a equidade dos dados, cujo padrão encontrado na área de cultivo irrigado de arroz, diferiu dos demais locais estudados. O número de espécies com 1 individuo nas áreas de mata primária e residual, foi em torno de 20 , ao passo que no arrozal foi 10 . Traduzindo esses números em porcentagem, a diferença diminui - na mata primária tais espécies representaram $24 \%$ do total na "Fonte" e $23 \%$ no Sítio Gentil; na mata A corresponderam a $16 \%$ e na B, $18 \%$; no arrozal, a $14 \%$. Levando-se em consideração o número de espécies com até 5 indivíduos as diferenças tornam-se mais nítidas: na "Fonte" isto representa $53 \%$ do total de espécies; no Sítio Gentil, 46,5\%; nas matas A e B, 36 e $41 \%$, respectivamente; e no arrozal, apenas $25 \%$. Por outro lado, a relação inverte-se quando se trata de espécies com mais de 1000 indivíduos: na "Fonte" elas representam apenas $2 \%$ do total e $3 \%$ no Sítio Gentil; na mata A 7,5\% e na $\mathrm{B}, 5,3 \%$; no arrozal este número sobe para $18 \%$. Deste modo, no arrozal a equidade é maior do que nos demais ambientes.

No que diz respeito aos índices, o de Margalef possui a vantagem de, através de cálculo simples, estabelecer relação entre $S$ e N. Falha, porém, na discriminação de dois ambientes com mesmos $\mathrm{S}$ e $\mathrm{N}$, mas diferentes equidades. Isso ocorre também com o indice de Fisher (MAGURRAN 1988; HAYEK e BUZAS 1997).

HAYEK e BUZAS (1997) propõem artificios para apurar a discriminação desses índices. Para o de Margalef, entende-se que seu valor máximo possivel refletiria situação na qual cada indivíduo representasse uma espécie (portanto, equidade máxima). Daí a expressão dmáx $=(\mathrm{N}-1) / \ln (\mathrm{N})$ e o índice padronizado (d/dmáx) passaria a incluir, ainda que teoricamente, a equidade. Além disso, a razão $\mathrm{S} / \mathrm{N}$ pode ajudar na discriminação de localidades se o índice padronizado der resultados próximos numericamente. Os autores alertam para casos em que $\mathrm{S}$ e $\mathrm{N}$ diferentes produzem o mesmo valor da razão $\mathrm{S} / \mathrm{N}$.

A razão $S / N$ consiste em ponto de partida para o cálculo do índice de Fisher $(\alpha)$. HAYEK e BUZAS (1997) explicam que o $\alpha$ seria um número próximo àquele esperado de espécies representadas por 1 indivíduo. $E$ como à medida em que $\mathrm{S}$ e $\mathrm{N}$ aumentam, também aumenta o número de espécies com 1 indivíduo, o $\alpha$ constitui-se numa boa medida de diversidade. Os autores sugerem o cálculo de $\mathrm{S}$ 
esperado $-S^{*}=-\alpha \ln (1-x)-$ e $N$ esperado $-N^{*}=\alpha x /(1-x)$ para avaliar se as estimativas são boas. Os cálculos foram efetuados e aproximaram-se bastante dos valores observados, quando não coincidiram com eles.

Olhando para as tabelas 1 e 2 dos resultados, de um modo geral, os indices comportaram-se semelhantemente. Os índices nas áreas de mata residual foram os maiores, seguidos dos de mata primária e, sempre menores, os da área de cultivo irrigado de arroz. Porém, para o índice de Margalef é dificil inferir diferenças significativas, principalmente para valores relativamente próximos. Contudo, segundo MAGURRAN (1988), este índice apresenta forte correlação com o $\alpha$ de Fisher que, por sua vez, permite o estabelecimento de intervalos de confiança. A não sobreposição de intervalos indica diferença significativa.

Verifica-se na tab.2 que há sobreposição dos intervalos de confiança dos dois pontos de mata primária, assim como dos pontos de mata residual. Portanto, a diversidade da Fonte e do Sítio Gentil não diferem entre si e a diversidade da mata A não difere da B. Seguindo o mesmo raciocínio, os três tipos de ambiente - mata primária, mata residual e área de cultivo irrigado de arroz - diferem significativamente quanto à diversidade.

Apenas para testar, porém sem interpretação, foram calculadas as variâncias de $\alpha$ através de duas fórmulas distintas, propostas por MAGURRAN (1988) e HAYEK e BUZAS (1997). Os valores diferiram muito entre si e foram sempre baixos, o que não seria esperado para dados como os aqui presentes.

ZANUNCIO e colaboradores (1998) compararam a diversidade de Lepidoptera em área de eucalipto com e sem faixas de vegetação nativa remanescente (cerrado), no Estado de Minas Gerais, e verificaram que na plantação homogênea a diversidade foi menor.

BRAGANÇA e colaboradores (1998) estudando Lepidoptera num transecto de $1000 \mathrm{~m}$ no Espírito Santo, iniciando-se em área de mata residual e terminando em plantação de eucalipto, verificaram maior riqueza de espécies e total de individuos na área de transição entre mata e plantação de eucalipto. Por outro lado, na mata residual a equidade foi maior (com $\mathrm{S}$ e $\mathrm{N}$ pouco inferiores ao da área de transição entre os ambientes). 
A discussão de tais contrastes está estreitamente relacionada com a dominância e a constância de espécies. Assim, segundo GASTON e LAWTON (1988), citados por ZANUNCIO e colaboradores (1998; p.89), as espécies mais comuns (distribuidas em mais ambientes) apresentam flutuações populacionais mais acentuadas e são capazes de explorar maiores quantidades de recursos do que as espécies raras.

Do ponto de vista da disponibilidade de recursos em gradientes de ambientes, numa plantação homogênea, a alta concentração de recursos associada ao baixo distúrbio, favorece algumas populações, as quais passam a ser dominantes. A riqueza de espécies tende a diminuir, bem como a diversidade.

Para ALTIERI e LETORNEAU (1984) e VANDERMEER (1989), citados por BRAGANÇA (1998), policulturas como florestas possuem maior heterogeneidade de recursos. No entanto, estes apresentam-se em menor concentração, o que limita o aumento das abundâncias, mas permite a adaptação de mais espécies. Entendendo recursos num contexto mais amplo, IQBAL e AUSTIN (1997) procuraram relacionar a riqueza de espécies hospedeiras com endemismo de seus parasitóides.

Vale lembrar que os trabalhos que acabaram de ser citados discutem diferenças entre mono e policulturas com vistas à conservação ambiental e/ou manejo de pragas. No que se refere à discussão sobre mosquitos, outros fatores devem ser considerados.

As fases imaturas e adultas de Culicídeos possuem nichos ecológicos distintos. As primeiras dependem da disponibilidade de água e representam a fase "vegetativa" da espécie, enquanto que as últimas dependem da disponibilidade de fontes sanguineas para a maturação dos ovos (e portanto, manutenção da espécie), representando a fase reprodutiva.

Deste modo, a associação de presença de mosquitos com ambientes não é tão direta quanto a relação herbivoria-vegetação. Interessa avaliar a disponibilidade de criadouros e preferências por fontes sanguíneas para repasto. Posto isto, torna-se possivel a compreensão das limitações inerentes às comparações de grupos distintos, exemplificada pela dificuldade de se obter um padrão de correlação entre precipitação média e frequência mensal das espécies dominantes. 
Nos pontos de mata primária, a espécie dominante foi Anopheles cruzii. No entanto, a diferença na valor do índice de Berger-Parker foi muito grande: 0,89 na "Fonte", com a espécie dominando em todos os meses nas mesmas proporções, e no Sítio Gentil, 0,36.

FORATTINI e colaboradores (1986a, 1986b) estudaram matas primárias no Vale do Ribeira e observaram que An. cruzii dominou sobre as demais espécies e esteve presente durante todos os meses do ano (nunca menos que $60 \%$ da fauna, chegando até a mais de $90 \%$ ), considerando diferentes métodos de captura em mata de planície e encosta. Nestas, o domínio da espécie foi mais expressivo. Os autores correlacionaram maior declividade, hábito acrodendrófilo e maior abundância. Este raciocínio pode ser aplicável a este estudo para a comparação entre "Fonte" e Sítio Gentil, de topografia menos acidentada.

As bromélias constituem o criadouro preferencial desta espécie e, de acordo com a classificação de FORATTINI (1962), são consideradas recipientes naturais permanentes ou semipermanentes. Estes vegetais existem abundantemente nas matas do Vale do Ribeira, tanto no solo quanto na copa das árvores. É possível que chuvas mais intensas façam transbordar a água desses criadouros e, com ela, as larvas do mosquito.

Nas áreas de mata residual a espécie dominante foi Aedes serratus (indice de Berger-Parker $=0,14$ na mata $A$ e 0,21 na $B$ ). $O N$ total foi muito próximo (4011 na mata $A$ e 3989 na $B$ ), contudo, sua distribuição mensal variou entre os locais e entre os anos no mesmo local. FORATTINI e colaboradores (1978a) já haviam apontado esta espécie como dominante em mata residual na região, com $46,3 \%$ do total de mosquitos. Essa diferença no grau de dominância pode ser explicada em função do esquema de captura: no trabalho referido foram realizadas das 9:00 às 11:00, das 16:00 às 18:00 e das 20:00 às 22:00 h, no solo e copa arbórea, enquanto que no presente trabalho as coletas limitaram-se ao período crepuscular $\mathrm{e}$ início da noite, somente no solo. Esta espécie é referida como diurna, embora FORATTINI e colaboradores (1981) tenham assinalado sua apreciável frequência em capturas noturnas.

As espécies do gênero Aedes ocorrem em criadouros transitórios, cuja existência é condicionada pelas chuvas (CONSOLI e LOURENÇO-DE-OLIVEIRA 
1994). Neste caso, não somente a intensidade das chuvas, mas também o intervalo de tempo entre as mesmas tornam-se fatores limitantes para o desenvolvimento das larvas até a fase adulta.

$\mathrm{Na}$ área de cultivo irrigado de arroz a espécie dominante foi Anopheles albitarsis (espécie B), com índice de Berger-Parker 0,35. Suas abundâncias foram muito discrepantes entre meses e anos. Na mesma região, FORATTINI e colaboradores (1993b, 1993c) acompanharam o ciclo do arroz, cuja fase de inundação coincidiu com o verão (quente e chuvoso) e verificaram que no sistema irrigado de arroz a densidade de larvas desta espécie foi 13 vezes maior do que em área natural. Concluíram que o período de inundação favoreceu diretamente o estabelecimento da espécie, tanto na sua fase imatura quanto adulta.

Além disso, FORATTINI e colaboradores (1994a) constataram que a produção de larvas foi inversamente proporcional à altura das plantas de arroz. Portanto, o favorecimento da espécie concentrou-se no início da fase de inundação.

Diante de todas essas informações, sabendo que o ciclo do arroz dura cerca de 150 dias, que seu início pode deslocar-se de um ano para outro (geralmente a fase de crescimento das plantas coincide com o verão) e que sempre há alternância de fases secas e de inundação, entende-se que Anopheles albitarsis requer criadouro permanente com pouca vegetação (pouco sombreados). A forte dependência de maiores quantidades de água justifica as flutuações mensais, mas não esclarece a amplitude das mesmas.

WALLNER (1987) em revisão sobre dinâmica populacional de insetos estudou espécies explosivas e não-explosivas. Embora tenha focalizado pragas agrícolas, o autor refere que populações de insetos, de um modo geral, flutuam mais em áreas secas do que em áreas úmidas (temperadas ou tropicais). Há muitos registros de flutuações de temperatura e precipitação que precederam explosões populacionais de insetos, mas o mecanismo exato de ação desse estresse ambiental permanece desconhecido.

Outro atributo de comunidades é a constância de espécies. A fórmula apresentada por DAJOZ (1973) foi aplicada para os dados, que estão representados nas figuras do item 4 do capitulo anterior. $O$ padrão de distribuição de espécies constantes, acessórias e acidentais foi nitidamente diferente no arrozal. Com relação 
às áreas de mata, a "Fonte" destacou-se um pouco das outras, as quais assemelharamse mais.

Deve-se estar atento a dois fatores: o número de coletas interfere diretamente no resultado da fórmula. Por exemplo, no Sítio Gentil em 1995 foram considerados 4 meses de coleta. Assim sendo, nenhuma espécie seria acidental. $\mathrm{O}$ outro fator que existe, mas não está contemplado na fórmula é a abundância da espécie. Mas geralmente espécies acidentais foram também pouco abundantes e as de maior abundância foram constantes (além de todas as dominantes).

Os determinantes da constância das espécies nos gradientes de ambientes possuem diferenças fundamentais. Na mata primária a heterogeneidade espacial, aliada à estabilidade de produtividade e produção primária propiciam a formação de inúmeros microhabitats. As espécies tendem a ser especialistas e até endêmicas, porém com limitações para atingir maior abundância.

Em mata residual estão presentes os atributos de mata primária, ainda que em menor escala. Áreas deste tipo são mais limitadas espacialmente e, frequentemente, adjacentes a áreas de mata primária, secundária e/ou áreas altamente impactadas. Isso aumenta o distúrbio que, de acordo com PIANKA (1994), em níveis intermediários favorece a adaptação de espécies e promove aumento de diversidade.

De modo similar, ODUM (1988) chama a atenção para o efeito de borda - áreas de transição de ambientes com características de ambos, deixam à disposição maior heterogeneidade de recursos (ainda que em menor quantidade).

Particularmente nas áreas de mata residual estudadas neste trabalho, a contiguidade com áreas abertas, a proximidade com o sistema irrigado de arroz e a presença de caracteristicas de mata primária em grau variável são fatores que podem justificar a maior diversidade em comparação com as outras localidades estudadas.

Quanto ao arrozal, a alternância controlada de períodos de seca e inundação torna o local altamente previsível. Mesmo nos periodos de drenagem, a disponibilidade de água das chuvas propicia a formação de criadouros transitórios, característicos de algumas espécies, como Aedes scapularis, Culex mollis e Culex nigripalpus, conforme constataram FORATTINI e colaboradores (1994b).

O maior número de espécies constantes encontradas no arrozal pode ser explicado por esta previsibilidade ambiental. Associado a isso, a extrema 
homogeneidade local restringe os tipos de recursos, mas também os poucos que sejam, do ponto de vista qualitativo, estão disponíveis em grande quantidade.

Estudos sobre monoculturas concentram-se principalmente em técnicas para aumento de produtividade e controle de pragas associadas aos produtos cultivados. Tais pragas têm merecido atenção por afetarem diretamente a plantação e, consequentemente a economia do produto de interesse.

As tabelas 4 e 5 dos resultados evidenciam a diferença na composição da fauna culicidiana. O indice de Sorensen indica a proporção de espécies compartilhadas por dois ambientes. Em todas as combinações de localidades, o arrozal compartilhou menor número de espécies com as áreas de mata primária e residual do que estas entre si. Com as matas residuais houve maior quantidade de espécies compartilhadas, o que pode ser explicado pela proximidade das localidades, sobretudo na mata $\mathrm{A}$, praticamente equidistante da mata $\mathrm{B}$ e do arrozal;

Particularmente, o cultivo irrigado de arroz propiciou a adaptação de espécies de culicídeos que merecem atenção por serem de importância epidemiológica conhecida ou potencial.

Finalmente, destacando na discussão espécies de importância médica encontradas nas localidades estudadas, todas as espécies do gênero Anopheles listadas no item 6.1 dos resultados são consideradas vetoras de malária. As espécies do subgênero Nyssorhynchus são consideradas vetoras secundárias ou locais de malária, segundo CONSOLI e LOURENÇO-DE-OLIVEIRA (1994). Consultando novamente a tabela 4 dos resultados, nota-se que as espécies deste subgênero foram acidentais ou ausentes nas matas primária e residual, mas todas foram constantes no arrozal (exceto Anopheles triannulatus, que foi acessória). Vale lembrar que Anopheles albitarsis (espécie B) foi inclusive dominante neste tipo de ambiente.

Apesar de o homem não ser sua fonte sanguínea preferencial (CONSOLI e LOURENÇO-DE-OLIVEIRA 1994), esta espécie adaptou-se a uma condição criada pelo homem. O fato de FORATTINI e colaboradores (1994b, 1996b) terem encontrado este anofelino em baixas densidades no ambiente domiciliar não isenta o homem do risco de transmissão de malária por esta espécie, já que em função da atividade agrícola, o homem vai até ela. 
No entanto, no que se refere à transmissão de malária, as espécies do subgênero Kerteszia causam maior preocupação, pois são vetoras primárias de malária endêmica no sul e sudeste do Brasil. $80 \%$ dos casos de malária autóctone no Estado de São Paulo, de 1990 a 1998, foram registrados no Vale do Ribeira e a transmissão foi atribuída às espécies de Kerteszia (SUCEN, dados não publicados).

Ao contrário dos anofelinos de Nyssorhynchus, as espécies de Kerteszia foram constantes nas áreas de mata primária e Anopheles cruzii, dominante. Segundo CONSOLI e LOURENÇO-DE-OLIVEIRA (1994), sua densidade populacional está diretamente relacionada com a abundância de bromélias e elevados índices de umidade relativa do ar, condições existentes nas matas primárias do Vale do Ribeira e que explicam sua abundância local. Porém, o que aumenta sua capacidade vetorial é o comportamento oportunista e eclético com relação à hematofagia. Além disso, FORATTINI e colaboradores (1993a, 1996a, 1999) indicaram a presença de Anopheles bellator e Anopheles cruzii em ambiente domiciliar, destacando naquela espécie maior endofagia e taxa de paridade.

Diante disso, tais espécies merecem acompanhamento, uma vez que, principalmente Anopheles bellator tem-se adaptado ao ambiente domiciliar e, ao mesmo tempo, o homem tem ocupado áreas de mata, impactando-as em grau variável.

BERGE (1975) indicou registros de Anopheles cruzii infectados por virus Bunyaviridae.

Com relação às espécies do gênero Aedes, todas as mencionadas neste trabalho estão associadas à transmissão de arboviroses. Destacaram-se Aedes scapularis e Aedes serratus, ambas espécies constantes em todos os ambientes (exceto Aedes serratus no Sítio Gentil, onde foi acessória), sendo esta dominante nas áreas estudadas de mata residual. Além disso, essas duas espécies são as mais comumente encontradas infectadas por arbovirus (Alfavirus, Flavivirus e Bunyavirus, segundo BERGE (1975)).

FORATTINI (1961) já na década de 60 preocupava-se com a domiciliação de Aedes scapularis na região do Vale do Ribeira e, em pesquisas posteriores, ele e sua equipe têm encontrado a espécie em ambiente domiciliar (1978b, 1987a, 1987b, 1989a, 1990, 1993d). Nestas pesquisas, outras espécies 
igualmente importantes pertencem ao gênero Culex - Culex ribeirensis e Culex sacchettae, ambas constantes em todos os ambientes aqui estudados. Estas três espécies foram incriminadas como possiveis vetoras do vírus Rocio durante a epidemia de encefalite por este agente no Vale do Ribeira em 1975-1976. Perseverando nas investigações, FORATTINI e colaboradores (1995b) incriminaram Aedes scapularis como vetor deste vírus.

Quanto aos demais Culex, todos foram acidentais ou acessórios em todos os ambientes, exceto Culex nigripalpus que foi constante em todos os ambientes (salvo no Sítio Gentil, onde foi acessória) e, inclusive, dominante na mata A em 1992.

Os Culex e as espécies dos outros gêneros são relacionados com transmissão de arbovírus. Ainda que tenham ocorrido em baixa densidade e/ou que os vírus que elas veiculam não causem quadros mais graves ao homem e/ou tenham sido restritas às matas, convém monitorá-las. Isso se justifica em função da tendência do homem em desmatar áreas para ocupação, podendo inserir-se em ciclos enzoóticos. Ao mesmo tempo, o grau de impacto que o homem causa ao ambiente exigirá das populações de mosquitos respostas rápidas no sentido de adaptar-se às novas condições impostas e à presença do próprio homem. 


\section{CONSIDERAÇÕES FINAIS}

A Região do Vale do Ribeira concentra características que permitem investigar processos bio-ecológicos e apontar tendências evolutivas das populações de culicídeos. Entre elas, destacam-se a proximidade de áreas representativas de floresta tropical úmida (comunidade clímax), matas residuais, matas secundárias, áreas de mangue, de restinga, rurais e áreas de ambiente tipicamente antrópico. Esse gradiente de ambientes reflete diferentes graus de impacto. $\mathrm{E}$, do ponto de vista de saúde pública, a transmissão endêmica de malária e o episódio epidêmico de encefalite por virus Rocio.

Estudos sobre biodiversidade geralmente estão atrelados à conservação de espécies, sobretudo aquelas ameaçadas de extinção. Estudos sobre mosquitos buscam conhecer esses insetos para melhor compreensão da dinâmica de doenças por eles transmitidas e, em última instância, para estabelecimento de medidas de controle dessas populações.

Neste trabalho procurou-se conciliar essas duas idéias na tentativa de dimensionar melhor a complexidade do problema das doenças transmitidas por mosquitos. A diversidade maior na mata residual atribui-se aqui à presença de características de mata primária e à proximidade de áreas alteradas (no caso, arrozal). A heterogeneidade espacial parece refletir-se na diversidade de espécies.

No arrozal a diversidade foi menor, mas a proporção de espécies constantes foi maior do que nos outros locais estudados, o que evidencia seleção de alguns culicídeos ao ponto de nem ocorrerem em áreas de mata.

Espécies raras, que em táxons superiores geralmente são encaradas como em vias de extinção, em se tratando de mosquitos, podem representar também espécies que estão se instalando no local. Contudo, o significado dessas espécies não se restringe apenas a essas duas situações. $O$ uso da armadilha tipo Shannon no horário crepuscular e início da noite pode ter "trasnformado" em raras, espécies diurnas, acrodendrófilas e/ou zoofilicas. De qualquer forma, o que se observou aqui foi que no arrozal as espécies raras ocorreram em menor número do que nas matas (cerca de 50\% inferior). 
Quanto à dominância, este trabalho mostrou que em cada tipo de ambiente dominou uma espécie diferente, todas de importância médica: na mata primária, Anopheles cruzii; na mata residual, Aedes serratus e, no arrozal, Anopheles albitarsis (espécie B).

O caráter dominante dessas espécies, juntamente com a tendência de aumento de impactos ambientais provocados pelo homem justificam a continuidade de pesquisas na região. Do ponto de vista de diversidade, o acompanhamento das áreas por mais tempo, o uso de outras técnicas e a inclusão de ambiente domiciliar complementariam este estudo. 


\section{REFERÊNCIAS BIBLIOGRÁFICAS}

Baltanás A. On the use of some methods for the estimation of species richness. Oikos 1992; 65: 484-92.

Baxter RM. Environmental effects of dams and impoundments. Ann. Rev. Ecol. Syst. 1977; 8: 255-83.

Begon M, Harper JL, Townsend CR. Ecology: individuals, populations and communities. $3^{\mathrm{a}}$ ed. Oxford: Blackwell Science; 1996.

Berge TO. International catalogue of arboviruses including certain other viruses of vertebrates. $2^{a}$ ed. DHEW Publication $n^{\circ}$.(CDC) 75-8301. 1975

Bragança M, DeSouza $\mathrm{O}$, Zanuncio JC. Environmental heterogeneity as a strategy for pest management in Eucalyptus plantations. Forest ecology and management 1998; 102: 9-12.

Consoli RAGB, Lourenço-de-Oliveira R. Principais mosquitos de importância sanitária no Brasil. Rio de Janeiro: Editora Fiocruz; 1994.

Coosemans M.; Mouchet J. Consequences of rural development on vectors and their control. Ann. Soc. Belge Med. Trop. 1990; 7: 5-23.

Dajoz R. Ecologia geral. Trad.de Guimarães FM. Petrópolis: Ed. Vozes/ Ed. Da USP; 1973;

DeFoliart GR, Grimstad PR, Watts DM. Advances in mosquito-borne arbovirus/vector research. Ann Rev Entomol. 1987; 32: 497-505.

Dobyns JR. Effects on sampling intensity on the collection of spider (Aranae) sprecies and the estimation of species richness. Environ Entomol. 1997; 26(2):150-62.

Forattini OP. Some data on the domesticity of Aedes scapularis (Rondani) in São Paulo, Brazil. Mosq News. 1961; 21(4): 295-6.

Forattini OP. Entomologia Médica. São Paulo: Edusp. 1962; v.1

Forattini OP. Entomologia Médica. São Paulo: Edusp. 1965a; v.2.

Forattini OP. Entomologia Médica. São Paulo: Edusp. 1965b; v.3

Forattini OP, Lopes OS, Rabello EX. Investigações sobre o comportamento de formas adultas de mosquitos silvestres no Estado de São Paulo, Brasil. Rev Saúde Pública. 1968; 2(2):111-76. 
Forattini OP, Gomes AC, Galati EAB, Rabello EX, Iverson LB. Estudos ecológicos sobre mosquitos Culicidae no Sistema da Serra do Mar, Brasil. 1- Observações no ambiente extradomiciliar. Rev Saúde Pública. 1978a; 12: 297-325.

Forattini OP, Gomes AC, Galati EAB, Rabello EX, Iverson LB. Estudos ecológicos sobre mosquitos Culicidae no Sistema da Serra do Mar, Brasil. 2- Observações no ambiente domiciliar. Rev Saúde Pública. 1978b; 12: 476-96.

Forattini OP, Gomes AC, Santos JLF, Galati EAB, Rabello EX, Natal D. Observações sobre atividade de mosquitos Culicidae, em mata residual no Vale do Ribeira, S. Paulo, Brasil. Rev Saúde Pública. 1981; 15: 557-86.

Forattini OP, Gomes AC, Natal D, Santos JLF. Observações sobre atividade de mosquitos Culicidae em mata primitiva da encosta no Vale do Ribeira, São Paulo, Brasil. Rev Saúde Pública. 1986a; 20(1): 1-20.

Forattini OP, Gomes AC, Natal D, Santos JLF. Observações sobre atividade de mosquitos Culicidae em matas primitivas da planicie e perfis epidemiológicos de vários ambientes no Vale do Ribeira, São Paulo, Brasil. Rev Saúde Pública. 1986b; 20(3): 178-203.

Forattini OP, Gomes AC, Natal D, Kakitani I, Marucci D. Preferências alimentares de mosquitos Culicidae no Vale do Ribeira, São Paulo, Brasil. Rev Saúde Pública. 1987a; 21(3): 171-87.

Forattini OP, Gomes AC, Natal D, Kakitani I, Marucci D. Freqüência domiciliar e endofilia de mosquitos Culicidae no Vale do Ribeira, São Paulo, Brasil. Rev Saúde Pública. 1987b; 21(3): 188-92.

Forattini OP, Gomes AC, Natal D, Kakitani I, Marucci D. Preferências alimentares de mosquitos Culicidae no Vale do Ribeira, São Paulo, Brasil, com especial referência a Aedes scapularis e a Culex (Melanoconion). Rev Saúde Pública. 1989a; 23(1): 9-19.

Forattini OP, Gomes AC, Natal D, Kakitani I. Observações sobre mosquitos Culicidae adultos em cultivo irrigado de arroz no Vale do Ribeira, São Paulo, Brasil. Rev Saúde Pública. 1989b; 23(4): 307-12.

Forattini OP, Gomes AC, Santos JLF, Kakitani I, Marucci D. Frequência ao ambiente humano e dispersão de mosquitos Culicidae em área adjacente à mata atlântica primitiva da planície. Rev Saúde Pública. 1990; 24(2): 101-7. 
Forattini OP . Ecologia, epidemiologia e sociedade. São Paulo: Edusp, 1992

Forattini OP, Kakitani I, Massad E, Gomes AC. Studies on mosquitoes (Diptera:

Culicidae) and anthropic environment. 1 - Parity of blood seeking Anopheles

(Kerteszia) in South-Eastem Brazil. Rev Saúde Pública. 1993a; 27(1): 1-8.

Forattini OP, Kakitani I, Massad E, Marucci D. Studies on mosquitoes (Diptera: Culicidae) and anthropic environment. 2 - Immature stages research at a rice irrigation system location in South-Eastern Brazil. Rev Saúde Pública. 1993b; 27(4): 227-36.

Forattini OP, Kakitani I, Massad E, Marucci D. Studies on mosquitoes (Diptera: Culicidae) and anthropic environment. 3 - Survey of adult stages at the rice irrigation system and the emergence of Anopheles albitarsis in South-Eastern, Brazil. Rev Saúde Pública. 1993c; 27(5): 313-25.

Forattini OP, Kakitani I, Massad E, Marucci D. Studies on mosquitoes (Diptera: Culicidae) and anthropic environment. 4 - Survey of resting adults and synanthropic behaviour in South-Eastern Brazil. Rev Saúde Pública. 1993d; 27 (6): $398-411$.

Forattini OP, Kakitani I, Massad E, Marucci D. Studies on mosquitoes (Diptera: Culicidae) and anthropic environment. 5 - Breeding of Anopheles albitarsis in flooded rice fields in South-Eastern Brazil. Rev Saúde Pública. 1994a; 28(5): 329-31.

Forattini OP, Kakitani I, Massad E, Marucci D. Studies on mosquitoes (Diptera: Culicidae) and anthropic environment. 6- Breeding in empty conditions of rice fields in South-Eastern Brazil. Rev Saúde Pública. 1994b; 28(6): 395-9.

Forattini OP, Kakitani I, Massad E, Marucci D. Studies on mosquitoes (Diptera: Culicidae) and anthropic environment. 7 - Behaviour of adults Nyssorhynchus anophelines with special reference to Anopheles albitarsis s.1. in South-Eastern Brazil. Rev Saúde Pública. 1995a; 28(1): 20-6.

Forattini OP, Kakitani I, Massad E, Marucci D. Studies on mosquitoes (Diptera: Culicidae) and anthropic environment. 9 - Synanthropy and epidemiological vector role of Aedes scapularis in South-Eastern Brazil. Rev Saúde Pública. 1995b; 29(3): 199-207. 
Forattini OP, Kakitani I, Massad E, Marucci D. Studies on mosquitoes (Diptera: Culicidae) and anthropic environment. 11 - Biting activity and blood-seeking parity of Anopheles (Kerteszia) in South-Eastern Brazil. Rev Saúde Pública. 1996a; 30(2): 107-14.

Forattini OP, Kakitani I, Massad E, Marucci D. Studies on mosquitoes (Diptera: Culicidae) and anthropic environment. 12 - Host-seeking behaviour of Anopheles albitarsis s.l. in South-Eastern Brazil. Rev Saúde Pública. 1996b; 30(4): 299. 303.

Forattini OP; Massad E. Culicidae vectors and anthropic changes in a South-Eastern Brazil natural ecosystem. Ecosystem Health 1998; 4(1): 9-19.

Forattini OP, Kakitani I, Santos RLC, Ueno HM, Kobayashi KM. Role of Anopheles (Kerteszia) bellator as malaria vector in Southeastern Brazil (Diptera: Culicidae). Mem Inst Oswaldo Cruz. 1999; 94(6): 715-8.

Gaston KJ. Biodiversity - a biology of numbers and difference. Oxford: Blackwell Science; 1996.

Granzow-de la Cerda Í, Zamora N, Vandermeer J, Boucher D. Diversidade de especies arbóreas en el bosque tropical húmedo del Caribe nicaragënse siete años después del huracán Juana. Rev Biol Trop. 1997; 45(4): 1409-19.

Hayek LC, Buzas MA. Surveying natural populations. New York: Columbia University Press; 1997.

Hunter JM, Rey L, Scott D. Man-made lakes - man-made diseases. World Health Forum 1983; 4(2):177-82.

Iqbal $M$, Austin $A D$. Species richness and endemism of baeine wasps (Hymenoptera: Scelionidae) in Australia. Mem of the Museum of Victoria. 1997; 56(2): 455-9.

Kostrowicki AS. Synanthropization as a result of environmental transformations. Memorabilia Zool. 1982; 37: 3-10.

Landau D, Prowell D, Carlton CE. Intensive versus long-term sampling to assess Lepidopteran diversity in a Southern mixed mesophitic forest. Ann Entomol Soc Am. 1999; 92(3): 435-41.

Magurran AE. Ecological diversity and its measurement. Cambridge: University Press, 1988. 
Odum. EP. Ecologia. Trad. de Tibe CJ. Rio de Janeiro: Guanabara Koogan; 1988.

Pianka ER. Evolutionary ecology. $5^{\text {a }}$ ed. New York: Harper Collins College Publishers; 1994.

Povolný D. Synanthropy. In: Greenberg B. Flies and disease. Volume I - Ecology, classification and biotic associations. New Jersey: Princeton University Press; 1971.

Rachou RG, Lima MM, Ferreira Neto JA, Martins CM. Alguns dados sobre o comportamento de mosquitos de Ponta Grossa (Florianópolis, Santa Catarina).

Rev Bras Malariol Doenças Trop. 1958; 10:417-27.

Schofield CJ. Environmental change - description or prediction? Ann. Soc. Belge Med. Trop. 1990; 70: 1-3.

Service MW. Rice, a challenge to health. Parasitol. Today 1989; 5(5): 162-5.

Service MW. Mosquito ecology - field sampling methods. $2^{a}$.ed. Londres: Chapman \& Hall; 1993.

Swengel $\mathrm{AB}$. Comparisons of butterfly richness and abundance measures in prairie and barrens. Biodiv Conserv. 1998; 7: 1639-59.

Teodoro U, Guilherme ALF, Lozovei AL, Salvia Filho VL, Sampaio AA, Spinosa RP, Ferreira MEMC, Barbosa OC, Lima EM. Mosquitos de ambientes peri e extradomiciliares na região sul do Brasil. Rev Saúde Pública. 1994; 28(2): 107 15.

Teodoro U, Guilherme ALF, Lozovei AL, Salvia Filho VL, Fukushigue Y, Spinosa RP, Ferreira MEMC, Barbosa OC, Lima EM. Culicídeos do lago de Itaipu, no rio Paraná, sul do Brasil. Rev Saúde Pública. 1995; 29(1): 6-14.

Wallner WE. Factors affecting insect population dynamics: differences between outbreak and non-outbreak species. Ann Rev Entomol. 1987; 32: 317-40.

Walsh JF, Molyneux DH, Birley MH. Deforestation: effects on vector-borne disease. Parasitology. 1993; 106: S55-75.

Zanuncio JC, Mezzomo JA, Guedes RNC, Oliveira AC. Influence of strips of native vegetation on Lepidoptera associated with Eucalyptus cloeziana in Brazil. Forest Ecol Manage. 1998; 108: 85-90. 
APÊNDICE 
Tab.1: Número e frequência relativa (F) de individuos coletados em armadilha tipo Shannon em área de mata primária ("Fonte" - Cananéia, SP) no periodo de fevereiro de 1992 a janeiro de 1993.

\begin{tabular}{|c|c|c|c|c|c|c|c|c|c|c|c|c|c|}
\hline Espécie & fev & mar & $a b r$ & maio & jun & jul & ago & set & out & nov & $\operatorname{dez}$ & jan* & total $F(\%)$ \\
\hline Ae.ergyrothorax & 0 & 0 & 1 & 0 & 0 & 0 & 0 & 1 & 2 & 1 & 0 & 1 & 60,016 \\
\hline Ae.fulvus & 2 & 1 & 2 & 3 & 0 & 0 & 0 & 0 & 0 & 0 & 1 & 0 & 90,024 \\
\hline Ae.gr.Serrat(hast/oli) & 0 & 0 & 1 & 1 & 2 & 0 & 6 & 0 & 0 & 2 & 1 & 0 & 130,035 \\
\hline Ae.gr.Serrat(sen/nub) & 36 & 209 & 94 & 28 & 114 & 22 & 18 & 33 & 9 & 50 & 41 & 14 & $668 \quad 1,787$ \\
\hline Ae.hastatus & 0 & 0 & 1 & 0 & 0 & 0 & 0 & 0 & 1 & 0 & 0 & 0 & 20,005 \\
\hline Ae.oligopistus & 0 & 0 & 10 & 0 & 0 & 0 & 0 & 0 & 1 & 0 & 6 & 0 & 170,045 \\
\hline Ae.sp & 1 & 2 & 1 & 0 & 1 & 2 & 0 & 6 & 0 & 1 & 9 & 3 & $26 \quad 0,07$ \\
\hline Ae.scapularis & 14 & 24 & 28 & 7 & 14 & 3 & 25 & 4 & 12 & 25 & 13 & 24 & 1930,516 \\
\hline Aeserratus & 7 & 54 & 44 & 15 & 46 & 7 & 5 & 10 & 13 & 11 & 47 & 8 & 2670,714 \\
\hline An.(Ano.)eiseni & 1 & 0 & 0 & 0 & 0 & 0 & 0 & 0 & 0 & 0 & 0 & 0 & 10,003 \\
\hline An.(Ste.)kompi & 0 & 0 & 0 & 0 & 0 & 1 & 0 & 0 & 0 & 0 & 0 & 0 & 10,003 \\
\hline An. (Ste.)kompican. & 0 & 0 & 0 & 0 & 0 & 0 & 0 & 0 & 0 & 1 & 1 & 0 & 20,005 \\
\hline An.(Ste.)nimt & 1 & 0 & 1 & 2 & 4 & 0 & 0 & 0 & 0 & 1 & 0 & 0 & 90,024 \\
\hline An bellator & 337 & 63 & 145 & 112 & 133 & 11 & 36 & 23 & 64 & 96 & 196 & 19 & 12353,304 \\
\hline An.cruzii & 9105 & 1979 & 2352 & 2702 & 3579 & 463 & 988 & 1193 & 3637 & 1970 & 4904 & 406 & $33278 \quad 89,02$ \\
\hline An.evansae & 0 & 0 & 0 & 0 & 0 & 0 & 0 & 1 & 0 & 0 & 0 & 0 & 10,003 \\
\hline Angalvaoi & 0 & 0 & 0 & 1 & 0 & 0 & 0 & 1 & 0 & 0 & 0 & 0 & 20,005 \\
\hline An.gr.punctimac & 0 & 0 & 1 & 0 & 0 & 0 & 0 & 0 & 0 & 0 & 0 & 0 & 10,003 \\
\hline An.interme & 0 & 0 & 1 & 1 & 2 & 0 & 0 & 0 & 6 & 10 & 8 & 11 & 390,104 \\
\hline Anlutzii & 0 & 0 & 0 & 0 & 0 & 0 & 0 & 0 & 0 & 1 & 0 & 0 & 10,003 \\
\hline An.oswaldoi & 0 & 1 & 0 & 0 & 0 & 0 & 0 & 2 & 0 & 0 & 0 & 0 & 30,008 \\
\hline An.prox.m & 6 & 1 & 10 & 13 & 21 & 2 & 3 & 7 & 13 & 19 & 6 & 3 & 1040,278 \\
\hline An.strodei & 0 & 0 & 0 & 0 & 0 & 0 & 0 & 2 & 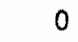 & 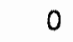 & 0 & 2 & 40,011 \\
\hline Cq.ch & 0 & 1 & 0 & 0 & 0 & 0 & 0 & 0 & 0 & 0 & 0 & 0 & 10,003 \\
\hline Cq.chrys/albifera & 14 & 18 & 10 & 3 & 0 & 0 & 0 & 0 & 0 & 9 & 20 & 2 & $76 \quad 0,203$ \\
\hline Cq.juxtaman & 0 & 0 & 0 & 0 & 0 & 0 & 0 & 0 & 0 & 0 & & & 20,005 \\
\hline Cq.ve & 0 & 1 & 1 & 0 & 0 & 0 & 0 & 0 & 0 & 0 & 1 & 0 & 30,008 \\
\hline CX.(Cux.)gr Coron. & 1 & 3 & 1 & 0 & 4 & 0 & 1 & 0 & 4 & 3 & 1 & 3 & 210,056 \\
\hline Cx. (Cux.)dec & 0 & 0 & 0 & 0 & 0 & 0 & 0 & 0 & 1 & 0 & 0 & 0 & 10,003 \\
\hline Cx.(Cux.).yggrus & 1 & 0 & 0 & 0 & 0 & 0 & 0 & 0 & 0 & 0 & 0 & 0 & 10,003 \\
\hline Cx. (Cux.)mollis & 1 & 4 & 0 & 0 & 0 & 0 & 0 & & 3 & 0 & & 0 & $14 \quad 0,037$ \\
\hline Cx.(Cux.)nignipalpus & 0 & 1 & 4 & 8 & 47 & 2 & 2 & 0 & 2 & 6 & 0 & 0 & 720,193 \\
\hline Cx. $(C u x) s p$. & 13 & 9 & 4 & 6 & 6 & 4 & 1 & 4 & 8 & 0 & 11 & $c$ & 660,177 \\
\hline Cx.(Cux) jusquatus & 0 & 1 & 0 & 0 & 0 & 1 & 0 & 0 & 0 & 0 & 0 & 0 & 20,005 \\
\hline$C x .(M c x$.$) tanei$ & 1 & 0 & 0 & 0 & 0 & 0 & 0 & 0 & 0 & 0 & 0 & 0 & 10,003 \\
\hline Cx.(Mcx.)dubitans & 0 & 0 & 0 & 0 & 0 & 0 & 0 & 0 & 0 & 0 & & 0 & 10,003 \\
\hline Cx.(Mcx.)imitator & 2 & 0 & 0 & 0 & 0 & 1 & 0 & 0 & 2 & 0 & 0 & 0 & 50,013 \\
\hline CX.(Mcx.)neglectus & 0 & 0 & 0 & 0 & 0 & 0 & 0 & 0 & 1 & 0 & 0 & 0 & 10,003 \\
\hline Cx.(Mcx.)pieunistriatus & 0 & 2 & 0 & 0 & 0 & 0 & 0 & 0 & 0 & 0 & 0 & 0 & 20,005 \\
\hline Cx.(Mel.jaliciae & 0 & 2 & 6 & 1 & 1 & 0 & 1 & 0 & 0 & 0 & 4 & 0 & $\begin{array}{ll}15 & 0.04\end{array}$ \\
\hline Cx.(Mel.)aureonotatus & 1 & 1 & 0 & 0 & 0 & 0 & 0 & 0 & 0 & 0 & 0 & 0 & 20,005 \\
\hline CX.(Mel.,bastagarius & 0 & 0 & 1 & 0 & 0 & 0 & 0 & 0 & 0 & 0 & 0 & 0 & 10,003 \\
\hline Cx.(Mel.)corentynensis & 1 & 1 & 4 & 0 & 0 & 1 & 0 & 1 & 0 & 0 & 0 & 0 & 80,021 \\
\hline
\end{tabular}


(cont.)

\begin{tabular}{|c|c|c|c|c|c|c|c|c|c|c|c|c|c|c|}
\hline Espécie & fev & mar & abr & maio & jun & jul & ago & set & out & nov & dez & $\mathrm{jan}^{\star}$ & total & $F(\%)$ \\
\hline$\overline{C x}($ Mel.)distinguendus & 1 & 0 & 0 & & 1 & 0 & 0 & 0 & 0 & 0 & 0 & 0 & 2 & 0,005 \\
\hline Cx.(Mel.)evansae & 2 & 0 & 0 & 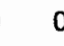 & 0 & 0 & 0 & 0 & 0 & 0 & 0 & 0 & 2 & 0,005 \\
\hline Cx.(Mel.)gaivaoi & 3 & 0 & 4 & 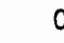 & 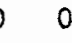 & 0 & 0 & 0 & 4 & 0 & 2 & 0 & 13 & 0,035 \\
\hline Cx.(Mel.)gnupo Atratus & 1 & 5 & 8 & 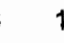 & 3 & 0 & 0 & 0 & 2 & 2 & 3 & 0 & 25 & 0,067 \\
\hline Cx.(Mel.)gr Intrincatus & 0 & 0 & 1 & 0 & 0 & 0 & 0 & 0 & 0 & 0 & 0 & 0 & 1 & 0,003 \\
\hline Cx.(Mel.)gnipo Pilosus & 0 & 1 & 0 & 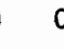 & 0 & 0 & 0 & 0 & 3 & 1 & 0 & 0 & 5 & 0,013 \\
\hline Cx,(Mel.)misionensis & 0 & 0 & 0 & 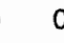 & 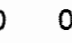 & 1 & 0 & 0 & 0 & 0 & 0 & 0 & 1 & 0,003 \\
\hline Cx (Mel.)ocellatus & 2 & 2 & 3 & c & 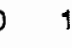 & 0 & 0 & 0 & 0 & 0 & 2 & 0 & 10 & 0,027 \\
\hline Cx.(Mel.)oedipus & 0 & 2 & 0 & 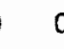 & 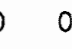 & 0 & 0 & 1 & 0 & 0 & 1 & 0 & 4 & 0,011 \\
\hline CX.(Mel.jpedroi & 0 & 0 & 2 & c & 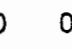 & 0 & 0 & 0 & 0 & 0 & 0 & 0 & 2 & 0,005 \\
\hline CX.(Mel.)pilosus & 0 & 0 & 0 & 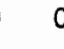 & 0 & 0 & 0 & 0 & 0 & 2 & 0 & 0 & 2 & 0,005 \\
\hline CX.(Mel.)plectoporpe & 0 & 0 & 0 & 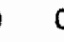 & 0 & 0 & 0 & 0 & 1 & 0 & 0 & 0 & 1 & 0,003 \\
\hline Cx.(Mel.)ribeirensis & 0 & 0 & 1 & 0 & 0 & 2 & 3 & 16 & 6 & 0 & 5 & 0 & 34 & 0,091 \\
\hline CX.(Mel.)sacchettae & 4 & 0 & 0 & 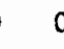 & 13 & 7 & 35 & 6 & 16 & 91 & 65 & 36 & 273 & 0,73 \\
\hline CX.(Mel.)taeniopus & 0 & 0 & 0 & 0 & 0 & 0 & 1 & 0 & 4 & 2 & 1 & 1 & 9 & 0,024 \\
\hline Cx.(Mel.)theobaldi VF & 3 & 3 & 0 & 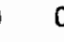 & 0 & 0 & 0 & 0 & 2 & 0 & 13 & 0 & 21 & 0,056 \\
\hline CX.(Mel.)zeteki & 0 & 0 & 0 & 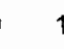 & 0 & 0 & 0 & 0 & 2 & 0 & 1 & 0 & 4 & 0,011 \\
\hline Li.durhami & 1 & 0 & 0 & c & 0 & 0 & 0 & 8 & 1 & 1 & 0 & 0 & 12 & 0,032 \\
\hline Liffavisetosus & 4 & 0 & 0 & 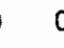 & 0 & 0 & 0 & 0 & 0 & 3 & 0 & 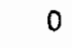 & 7 & 0,019 \\
\hline Ma.(Man.)sp & 0 & 0 & 0 & 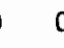 & 0 & 0 & 0 & 0 & 0 & 0 & 1 & ( & 1 & 0,003 \\
\hline Ph.pallidiventer & 3 & 0 & 0 & 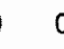 & 0 & 0 & 0 & 0 & 3 & 0 & 0 & & 6 & 0,016 \\
\hline Ph.prox.fiabellata & 1 & 0 & 0 & 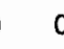 & 0 & 0 & 0 & 0 & 0 & 0 & 0 & 0 & 1 & 0,003 \\
\hline Ph.prox.longirostris & 2 & 0 & 0 & 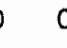 & 0 & 0 & 0 & 0 & 0 & 0 & 0 & & 2 & 0,005 \\
\hline Ph.prox.palmata/diabo & 2 & 0 & 0 & 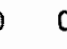 & 0 & 0 & 0 & 0 & 3 & 0 & 3 & 0 & 8 & 0,021 \\
\hline Ph.prox. theobaidi & 8 & 0 & 0 & 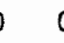 & 0 & 0 & 0 & 0 & 0 & 0 & 0 & & 8 & 0,021 \\
\hline Ph.davisi & 1 & 0 & 0 & 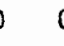 & 0 & 0 & 0 & 0 & 0 & 0 & 0 & & 1 & 0,003 \\
\hline Ph.flabellata & 0 & 1 & 0 & 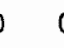 & 0 & 1 & 0 & 0 & 4 & 1 & 7 & 2 & 18 & 0,048 \\
\hline Ph.longirostris & 0 & 0 & 0 & 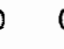 & 0 & 0 & 0 & 0 & 0 & 0 & 1 & & 2 & 0,005 \\
\hline Ph.palmata/diabolica & 1 & 0 & 0 & 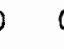 & 0 & 0 & 1 & 0 & 1 & 3 & 3 & 3 & 14 & 0,037 \\
\hline Ph.quasilongirostris & 2 & 0 & 0 & 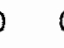 & 0 & 0 & 0 & 0 & 1 & 2 & 0 & 0 & 5 & 0,013 \\
\hline Ph.the & 0 & 1 & 0 & & 0 & 0 & 3 & 0 & 7 & 2 & 1 & 0 & 14 & 0,037 \\
\hline Ps. albigenu & 0 & 0 & 0 & 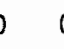 & 0 & 0 & 0 & 0 & 0 & 0 & 48 & 0 & 48 & 0,128 \\
\hline Ps.ferox & 7 & 5 & 5 & 5 & 0 & 1 & 0 & 0 & 0 & 6 & 215 & $\theta$ & 248 & 0,663 \\
\hline Ps.futzii & 1 & 10 & 1 & 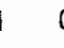 & 0 & 0 & 0 & 0 & 0 & 0 & 9 & 0 & 21 & 0,056 \\
\hline Ru.numboldt & 0 & 1 & 0 & & 0 & 0 & 0 & 0 & 0 & 1 & 0 & 0 & 3 & 0,008 \\
\hline Ru.prox.re & 2 & 8 & 1 & & 0 & 0 & 0 & 3 & 0 & 0 & 0 & 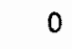 & 14 & 0,037 \\
\hline Ru.reversa & 7 & 0 & 2 & & 0 & 6 & 1 & 11 & 6 & 10 & 18 & 1 & 64 & 0,171 \\
\hline Ru.theobaldi & 0 & 0 & 0 & & 0 & 0 & 0 & 0 & 0 & 1 & 0 & 0 & 1 & 0,003 \\
\hline Sh.fuviatilis & 0 & 0 & 1 & & 0 & 0 & 0 & 0 & 0 & 0 & 0 & 0 & 1 & 0,003 \\
\hline Tr.pollid/castroi/similis & 8 & 1 & 0 & & 0 & 0 & 0 & 0 & 0 & 0 & 0 & 0 & 9 & 0,024 \\
\hline Tr.digitatum & 0 & 0 & 0 & & 0 & 0 & 0 & 0 & 2 & 0 & 0 & 0 & 2 & 0,005 \\
\hline Ur.calosomata & 0 & 0 & 0 & & 0 & 0 & 0 & 0 & 0 & 2 & 0 & 0 & 3 & 0,008 \\
\hline Ur.geometrica & 0 & 0 & 0 & & 0 & 0 & 0 & 0 & 0 & 0 & 1 & 0 & 1 & 0,003 \\
\hline Ur.mathesoni & 0 & 0 & 0 & & 1 & 0 & 0 & 0 & 0 & 0 & 0 & 0 & 1 & 0,003 \\
\hline Wy.felicia/pampeites & 0 & 0 & 0 & & 0 & 0 & 0 & 0 & 0 & 0 & 8 & 0 & 8 & 0,021 \\
\hline Wy.sp & 3 & 0 & 1 & 1 & 1 & 0 & 0 & 0 & 2 & 0 & 2 & 0 & 9 & 0,024 \\
\hline Wy.aporonoma & 1 & 0 & 1 & 1 & 0 & 0 & 0 & 0 & 0 & 0 & 0 & 0 & 2 & 0,005 \\
\hline
\end{tabular}


(cont.)

\begin{tabular}{lrrrrrrrrrrrrrrr}
\hline Espécie & jan & fev & mar & abr maio & jun & jul & ago & set & out & nov & dez & total & $F(\%)$ \\
\hline Wy.confusa & 28 & 10 & 11 & 11 & 9 & 47 & 13 & 15 & 30 & 13 & 45 & 0 & 232 & 0,621 \\
Wy.finlayi & 1 & 0 & 0 & 0 & 0 & 0 & 0 & 0 & 0 & 0 & 0 & 0 & 1 & 0,003 \\
Wy.mystesffin! & 0 & 0 & 3 & 0 & 2 & 0 & 1 & 0 & 3 & 2 & 8 & 2 & 21 & 0,056 \\
Wy.shannoni & 2 & 0 & 0 & 0 & 2 & 2 & 0 & 3 & 0 & 0 & 0 & 0 & 9 & 0,024
\end{tabular}

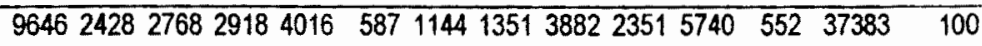

janeiro/93

Tab.2: Número frequência relativa $(\mathrm{F})$ de individuos coletados em armadilha tipo Shannon em área de mata primária (Sítio Gentil - Cananéia) de junho a dezembro de 1993

\begin{tabular}{|c|c|c|c|c|c|c|c|c|c|}
\hline Espécie & jun & jul & 290 & set & out & nov & $\operatorname{dez}$ & total & $F(\%)$ \\
\hline Ae.ergyrothorax & 0 & 0 & 0 & 0 & 2 & 0 & 0 & 2 & 0,03 \\
\hline Ae.gr.Serrat(hastoli) & 0 & 0 & 0 & 0 & 2 & 0 & 1 & 3 & 0,04 \\
\hline Ae.gr.Serrat(sen/nub) & 0 & 0 & 0 & 0 & 2 & 3 & 13 & 18 & 0,24 \\
\hline Ae.scapularis & 4 & 2 & 0 & 2 & 58 & 3 & 8 & 77 & 1,02 \\
\hline Ae.serratus & 0 & 0 & 0 & 0 & 6 & 0 & 31 & 37 & 0,49 \\
\hline An.bellator & 57 & 347 & 54 & 166 & 726 & 184 & 265 & 1799 & 23,9 \\
\hline An.cruzï & 155 & 297 & 202 & 290 & 1051 & 253 & 358 & 2606 & 34,6 \\
\hline An.prox.mediopunctatus - & 0 & 0 & 0 & 0 & 0 & 1 & 0 & 1 & 0,01 \\
\hline Cq.chrysonotum & 0 & 0 & 0 & 0 & 15 & 7 & 3 & 25 & 0,33 \\
\hline Cq.chrysonotum/albifera & 5 & 0 & 0 & 0 & 144 & 2046 & 181 & 2376 & 31,6 \\
\hline Cq.hermanoi & 0 & 0 & 0 & 0 & 0 & 7 & 0 & 7 & 0,09 \\
\hline Cq.juxtamansonia & 0 & 0 & 0 & 0 & 0 & 18 & 230 & 248 & 3,29 \\
\hline Cq.nigricans & 0 & 0 & 0 & 0 & 0 & 2 & 0 & 2 & 0,03 \\
\hline Cq.venezuelensis & 2 & 0 & 0 & 0 & 4 & 51 & 22 & 79 & 1,05 \\
\hline Cx. (Cux.) gr Coronator & 0 & 0 & 3 & 0 & 0 & 7 & 0 & 10 & 0,13 \\
\hline Cx.(Cux.)dolosus & 0 & 0 & 0 & 1 & 0 & 0 & 0 & 1 & 0,01 \\
\hline Cx.(Cux.)nignipalpus & 1 & 0 & 0 & 0 & 0 & 0 & 0 & 1 & 0.01 \\
\hline CX.(Cux.)sp. & 1 & 1 & 0 & 0 & 10 & 9 & 11 & 32 & 0,42 \\
\hline$C_{x}$ (Lut.)bigoti & 0 & 0 & 0 & 0 & 1 & 0 & 0 & 1 & 0,01 \\
\hline Cx. $(M c x)$.neglectus & 0 & 0 & 0 & 1 & 0 & 0 & 0 & 1 & 0,01 \\
\hline$C_{x} .($ Mel.)corentynensis & 0 & 0 & 0 & 0 & 2 & 0 & 0 & 2 & 0,03 \\
\hline Cx.(Mel.)gatvaoi & 2 & 0 & 0 & 1 & 0 & 0 & 0 & 3 & 0,04 \\
\hline Cx.(Mel.)grupo Pilosus & 0 & 0 & 0 & 0 & 0 & 2 & 0 & 2 & 0.03 \\
\hline Cx.(Mel.)ocellatus & 0 & 2 & 0 & 3 & 0 & 1 & 0 & 6 & 0,08 \\
\hline Cx.(Mel.)ocosse & 0 & 0 & 0 & 0 & 0 & 2 & 0 & 2 & 0,03 \\
\hline Cx.(Mel.)pedroi & 0 & 0 & 0 & 1 & 0 & 0 & 0 & 1 & 0,01 \\
\hline Cx.(Mel.)ribeirensis & 0 & 0 & 2 & 3 & 3 & 18 & 15 & 41 & 0,54 \\
\hline Cx.(Mel.)sacchettae & 5 & 2 & 1 & 2 & 0 & 7 & 14 & 31 & 0,41 \\
\hline Cx.(Mel.)zeteki & 0 & 0 & 0 & 1 & 0 & 0 & 0 & 1 & 0,01 \\
\hline Lidurhami & 1 & 0 & 0 & 0 & 3 & 1 & 9 & 14 & 0,19 \\
\hline Lifflavisetosus & 1 & 0 & 1 & 0 & 0 & 0 & 0 & 2 & 0,03 \\
\hline Ma.(Man.)sp & 0 & 0 & 0 & 0 & 0 & 3 & 0 & 3 & 0,04 \\
\hline Ph.palmata/diabolica & 1 & 0 & 0 & 0 & 0 & 2 & 0 & 3 & 0,04 \\
\hline Ph.quasilongirostris & 0 & 0 & 0 & 0 & 1 & 13 & 3 & 17 & 0,23 \\
\hline Ps.albigenu & 0 & 0 & 0 & 0 & 5 & 0 & 0 & 5 & 0,07 \\
\hline
\end{tabular}




\begin{tabular}{lrrrrrrrrr} 
(cont.) & jun & jul & ago & set & out & nov & dez & total & $F(\%)$ \\
\hline Especie & 0 & 0 & 0 & 0 & 0 & 0 & 5 & 5 & 0,07 \\
\hline Ps.forox & 0 & 0 & 0 & 0 & 0 & 1 & 2 & 3 & 0,04 \\
Ru.reverse & 0 & 0 & 3 & 1 & 0 & 0 & 2 & 6 & 0,08 \\
Ur.calosomata & 2 & 3 & 0 & 10 & 0 & 0 & 0 & 15 & 0,2 \\
Ur.geometrica & 3 & 1 & 0 & 1 & 0 & 1 & 0 & 6 & 0,08 \\
Ur.pulchemima & 0 & 0 & 0 & 0 & 0 & 1 & 0 & 1 & 0,01 \\
Wy.felicialpampeites & 6 & 0 & 1 & 1 & 1 & 4 & 21 & 34 & 0,45 \\
Wy.confusa & 0 & 0 & 0 & 0 & 0 & 0 & 1 & 1 & 0,01 \\
Wy.mystes ou finlayi & 246 & 655 & 267 & 484 & 2036 & 2647 & 1195 & 7530 & 100 \\
\hline & & & & & & & & &
\end{tabular}

Tab.3: Número e frequência relativa (F) de individuos coletados em armadilha tipo Shannon em área de mata primária (Sitio Gentil - Cananéia) durante o ano de 1994.

\begin{tabular}{|c|c|c|c|c|c|c|c|c|c|c|c|c|c|}
\hline Espécie & jan & fev & mar & $a b r$ & maio & jun & jul & ago & set & out & nov & $\operatorname{dez}$ & total $F(\%)$ \\
\hline Ae.argyrothorax & 1 & 0 & 0 & 0 & 1 & 0 & 0 & 0 & 0 & 0 & 0 & 0 & 20,02 \\
\hline Ae.crinifer & 1 & 0 & 0 & 0 & 0 & 0 & 0 & 0 & 0 & 0 & 0 & 0 & 10,01 \\
\hline Ae.gr.Serrat(hastioli) & 1 & 0 & 1 & 0 & 1 & 0 & 0 & 0 & 0 & 0 & 0 & 0 & 30,03 \\
\hline Ae.gr.Serrat(serr/nub) & 20 & 2 & 6 & 4 & 4 & 2 & 0 & 0 & 0 & 0 & 0 & 0 & 380,35 \\
\hline Ae.hastatus & 0 & 0 & 0 & 0 & 1 & 0 & 0 & 0 & 0 & 0 & 0 & 0 & 10,01 \\
\hline Ae. oligopistus & 0 & 0 & 0 & 0 & 2 & 0 & 0 & 0 & 0 & 0 & 0 & 0 & 20,02 \\
\hline Ae.sp & 1 & 0 & 0 & 0 & 0 & 0 & 0 & 1 & c & 0 & 0 & 0 & 20,02 \\
\hline Ae.scapularis & 1 & 14 & 6 & 17 & 6 & 6 & 2 & 1 & 0 & 6 & 22 & 33 & 1141,05 \\
\hline Ae.serratus & 10 & 2 & 5 & 4 & 0 & 0 & 0 & 0 & 0 & 0 & 0 & 0 & 210,19 \\
\hline An albitarsis esp. $B$ & 0 & 0 & 0 & 1 & 0 & 0 & 0 & 0 & 0 & 0 & 0 & 0 & 10,01 \\
\hline An.(Ste.)kompicanoni & 0 & 0 & 0 & 0 & 1 & 0 & 0 & 0 & 0 & 0 & 0 & 0 & 10,01 \\
\hline An.bellator & 242 & 657 & 565 & 113 & 748 & 23 & 10 & 72 & 18 & 333 & 79 & 338 & 319829,4 \\
\hline An.cruzii & 170 & 425 & 454 & 92 & 367 & 22 & 8 & 60 & 25 & 247 & 191 & 960 & 302127,8 \\
\hline An.prox.mediopunctatus & 0 & 0 & 0 & 0 & 1 & 0 & 0 & 0 & 0 & 0 & 0 & 0 & 10,01 \\
\hline Cq.chrysonotum & 2 & 2 & 0 & 0 & 26 & 0 & 0 & 0 & 1 & 29 & 12 & 17 & 890,82 \\
\hline Cq.chrysonotum/albifera & 90 & 74 & 22 & 134 & 259 & 2 & 0 & 0 & 53 & 872 & 1397 & 313 & 321629,6 \\
\hline Cq.juxtamansonia & 24 & 39 & 2 & 0 & 0 & 0 & 0 & 0 & 0 & 8 & 0 & 0 & 730,67 \\
\hline Cq.nigricans & 0 & 0 & 0 & 0 & 0 & 0 & 0 & 0 & 0 & 1 & 0 & 0 & 10,01 \\
\hline Cq.venezuelensis & 36 & 41 & 13 & 15 & 9 & 0 & 0 & 0 & 0 & 26 & 5 & 50 & $195 \quad 1,8$ \\
\hline Cx.(Cux.) gr Coronator & $\theta$ & 4 & 1 & 0 & 3 & 1 & 7 & 0 & 2 & 3 & 0 & 0 & 300.28 \\
\hline Cx.(Cux.)chidesteri & 0 & 0 & 0 & 0 & 0 & 1 & 0 & 0 & 0 & 0 & 0 & 0 & 10,01 \\
\hline Cx.(Cux.)mollis & 0 & 0 & 0 & 0 & 0 & 0 & 0 & 0 & 0 & 0 & 3 & 0 & 30,03 \\
\hline Cx.(Cux.)nignipalpus & 0 & 0 & 2 & 0 & 0 & 0 & 0 & 0 & 0 & 2 & 0 & 0 & 40.04 \\
\hline Cx.(Cux.)sp. & 0 & 4 & 0 & 12 & 51 & 8 & 9 & 7 & 0 & 4 & 13 & 0 & $108 \quad 0,99$ \\
\hline Cx.(Mcx.) lanei & 0 & 0 & 0 & 0 & 1 & 0 & 0 & 0 & 0 & 0 & 0 & 0 & 10,01 \\
\hline Cx.(Mcx.)grupo imitator & 0 & 0 & 0 & 0 & 0 & 0 & 0 & 0 & 0 & 0 & 0 & 1 & 10.01 \\
\hline Cx.(MCx.)imitator & 0 & 0 & 0 & 0 & 0 & 0 & 0 & 0 & 0 & 0 & 0 & 1 & 10.01 \\
\hline CX.(MCX.)neglectus & 0 & 0 & 0 & 0 & 0 & 0 & 0 & 0 & 0 & 1 & 0 & 0 & 10,01 \\
\hline Cx.(Mcx.)pleuristriatus & 0 & 0 & 0 & 0 & 0 & 0 & 0 & 0 & 0 & 0 & 0 & 1 & 10,01 \\
\hline Cx.(Mel.)bastagarius & 7 & 0 & 0 & 0 & 0 & 0 & 0 & 0 & 0 & 0 & 0 & 0 & 70,06 \\
\hline Cx.(Mel.)dunni & 0 & 0 & 0 & 0 & 1 & 0 & 0 & 0 & 0 & 0 & 1 & 0 & 20,02 \\
\hline Cx.(Mel.)evansae & 0 & 0 & 0 & 0 & 0 & 0 & 0 & 1 & 0 & 0 & 0 & 0 & 10,0 \\
\hline
\end{tabular}


(cont.)

\begin{tabular}{|c|c|c|c|c|c|c|c|c|c|c|c|c|c|}
\hline Espécie & jan & fev & mar & $a b r$ & maio & jun & jul & ago & set & out & nov & dez & total $F(\%)$ \\
\hline Cx.(Mel.)galvaoi & 0 & $\overline{0}$ & 0 & 0 & 7 & 0 & 0 & 1 & 0 & 0 & 0 & 0 & 80,07 \\
\hline CX.(Mel.)grupo Atratus & 0 & 0 & 0 & 0 & 0 & 0 & 0 & 0 & 0 & 0 & 0 & 2 & 20,02 \\
\hline Cx.(Mel.)gnupo Intrincatus & 0 & 0 & 0 & 0 & 0 & 0 & 0 & 0 & 0 & 0 & 1 & 0 & 10,01 \\
\hline $\mathrm{Cx}_{\mathrm{X}}($ Mel.)gnupo Pilosus & 2 & 1 & 0 & 0 & 1 & 0 & 0 & 0 & 0 & 0 & 125 & 0 & 1291,19 \\
\hline Cx.(Mel.)misionensis & 0 & 0 & 0 & 0 & 0 & 0 & 0 & 0 & 0 & 1 & 0 & 0 & 10,01 \\
\hline CX.(Mel.)ocoss 8 & 0 & 0 & 0 & 0 & 0 & 0 & 0 & 0 & 0 & 1 & 1 & 0 & 20,02 \\
\hline Cx.(Mel.)pedroi & 0 & 0 & 0 & 0 & 0 & 0 & 0 & 1 & 0 & 0 & 0 & 0 & 10,01 \\
\hline Cx.(Mel.)plectoporpe & 0 & 0 & 0 & 0 & 0 & 0 & 0 & 0 & 0 & 6 & 0 & 0 & 60,06 \\
\hline Cx.(Mel.)rabelloi & 0 & 0 & 0 & 0 & 0 & 0 & 0 & 0 & 0 & 1 & 0 & 1 & 20,02 \\
\hline Cx.(Mel.)nibeirensis & 21 & 0 & 6 & 1 & 1 & 1 & 0 & 0 & 1 & 0 & 0 & 4 & 350,32 \\
\hline CX.(Mel.)sacchettae & 1 & 5 & 5 & 12 & 17 & 6 & 0 & 3 & 4 & 10 & 16 & 28 & 1070,99 \\
\hline Cx.(Mel.)sp & 0 & 0 & 0 & 0 & 0 & 0 & 0 & 0 & 0 & 2 & 0 & 0 & 20,02 \\
\hline Cx.(Mel.)taeniopus & 0 & 0 & 0 & 1 & 0 & 0 & 1 & 0 & 0 & 0 & 0 & 0 & 20,02 \\
\hline Cx. (Mel.)theobaldi V. $R$. & 3 & 0 & 6 & 0 & 0 & 0 & 0 & 0 & 0 & 0 & 0 & 0 & 90,08 \\
\hline Cx.(Mel.)zeteki & 0 & 1 & 0 & 0 & 0 & 0 & 0 & 0 & 0 & 0 & 0 & 1 & 20,02 \\
\hline Lidumami & 25 & 5 & 0 & 8 & 0 & 0 & 0 & 0 & 3 & 7 & 2 & 5 & 550,51 \\
\hline Liflavisetosus & 0 & 2 & 0 & 1 & 4 & 0 & 0 & 0 & 0 & 0 & 1 & 0 & 80,07 \\
\hline Ma.(Man.Jindubitans & 0 & 0 & 0 & 0 & 0 & 0 & 0 & 0 & 0 & 3 & 0 & 0 & 30,03 \\
\hline Ma.(Man.jpseudotitillans & 0 & 0 & 0 & 0 & 0 & 0 & 0 & 0 & 0 & 3 & 1 & 0 & 40,04 \\
\hline Ma.(Man.)sp & 2 & 0 & 0 & 0 & 1 & 1 & 0 & 0 & 0 & 4 & 2 & 4 & 140,13 \\
\hline Ph.prox.palmata/diabolica & 0 & 5 & 1 & 3 & 0 & 0 & 0 & 0 & 0 & 0 & 0 & 0 & 90,08 \\
\hline Ph.prox.theobaldi & 0 & 0 & 3 & 4 & 0 & 0 & 0 & 0 & 0 & 0 & 0 & 0 & 70,06 \\
\hline Ph.flabellata & 0 & 0 & 0 & 0 & 0 & 0 & 0 & 1 & 0 & 0 & 0 & 0 & 10,01 \\
\hline Ph.palmata/diabolica & 3 & 0 & 0 & 3 & 0 & 0 & 0 & 0 & 15 & 0 & 0 & 5 & $26 \quad 0,24$ \\
\hline Ph.quasilongirostris & 22 & 6 & 1 & 2 & 0 & 0 & 0 & 0 & 0 & 0 & 0 & 1 & 320,29 \\
\hline Ph.theobaldi & 0 & 0 & 1 & 7 & 1 & 0 & 0 & 0 & 8 & 0 & 3 & 17 & 370,34 \\
\hline Phoniomyia sp & 0 & 0 & 0 & 1 & 0 & 0 & 0 & 0 & 0 & 0 & 0 & 0 & 10,01 \\
\hline Ps.albigenu & 26 & 0 & 0 & 0 & 0 & 0 & 0 & 0 & 0 & 0 & 0 & 0 & $26 \quad 0,24$ \\
\hline Ps.albipes & 10 & 0 & 0 & 0 & 0 & 0 & 0 & 0 & 0 & 0 & 0 & 0 & 100,09 \\
\hline Ps.ferox & 3 & 4 & 0 & 0 & 0 & 0 & 0 & 0 & 0 & 0 & 0 & 0 & 70,06 \\
\hline Ru.humboldt & 0 & 0 & 0 & 0 & 0 & 0 & 0 & 0 & 0 & 0 & 1 & 0 & 10,01 \\
\hline Ru.prox.reversa & 0 & 1 & 0 & 0 & 0 & 0 & 0 & 0 & 0 & 0 & 0 & 0 & 10,01 \\
\hline Ru.reversa & 2 & 0 & 0 & 0 & 2 & 0 & 2 & 0 & 0 & 1 & 1 & 1 & 90,08 \\
\hline Ru.theobaldi & 0 & 0 & 0 & 0 & 0 & 0 & 0 & 0 & 0 & 0 & 1 & 0 & 10,01 \\
\hline Ur.calosomata & 0 & 0 & 0 & 0 & 1 & 1 & 0 & 0 & 1 & 1 & 0 & 0 & 40,04 \\
\hline Ur.geometrica & 0 & 0 & 1 & 3 & 1 & 0 & 1 & 7 & 4 & 2 & 0 & 0 & 190,17 \\
\hline Ur.lowi & 0 & 0 & 0 & 0 & 0 & 0 & 0 & 0 & 0 & 0 & 0 & 1 & 10,01 \\
\hline Wy. felicia/pampeites & 1 & 1 & 1 & 5 & 0 & 0 & 0 & 0 & 1 & 14 & 2 & 0 & 250,23 \\
\hline Wy.confusa & 22 & 15 & 10 & 3 & 10 & 4 & 4 & 3 & 2 & 11 & 12 & 10 & $106 \quad 0,98$ \\
\hline Wy.shannoni & 0 & 0 & 0 & 1 & 0 & 0 & 1 & 0 & 0 & 0 & 0 & 1 & 30,03 \\
\hline
\end{tabular}


Tab.4: Nủmero e frequência relativa $(F)$ de indivíduos coletados em armadilha tipo Shannon em área de mata primária (Sítio Gentil Cananéia) de janeiro a abril de 1995.

\begin{tabular}{|c|c|c|c|c|c|c|}
\hline Espécie & jan & fev & mar & $a b r$ & total & $F(\%)$ \\
\hline A日.argyrothorax & 0 & 0 & 0 & 5 & 5 & 0,06 \\
\hline Ae.gr.Serrat(hast/oli) & 0 & 1 & 6 & 0 & 7 & 0,09 \\
\hline Ae.gr.Serrat(sem/nub) & 2 & 10 & 3 & 0 & 15 & 0,18 \\
\hline Ae.prox.perventor ou hortator & 1 & 0 & 1 & 0 & 2 & 0,02 \\
\hline Ae.scapularis & 121 & 104 & 199 & 30 & 454 & 5,54 \\
\hline Ae.serratus & 77 & 3 & 4 & 2 & 86 & 1,05 \\
\hline An.bellator & 264 & 921 & 594 & 356 & 2135 & 26 \\
\hline An.cnuzii & 1656 & 1213 & 867 & 315 & 4051 & 49,4 \\
\hline An.intermedius & 0 & 0 & 1 & 0 & 1 & 0,01 \\
\hline An.prox.mediopunctatus & 0 & 0 & 1 & 0 & 1 & 0,01 \\
\hline Cq.chrysonotum & 14 & 2 & 33 & 6 & 55 & 0,67 \\
\hline Cq.chnysonotum ou albifera & 74 & 58 & 441 & 56 & 629 & 7,67 \\
\hline Cq.venezuelensis & 22 & 15 & 26 & 9 & 72 & 0,88 \\
\hline Cx.(Cux.) gr Coronator & 0 & 4 & 6 & 10 & 20 & 0,24 \\
\hline Cx.(Cux.)bidens & 0 & 0 & 4 & 0 & 4 & 0,05 \\
\hline Cx.(Cux.)chidesteri & 0 & 0 & 2 & 0 & 2 & 0,02 \\
\hline Cx.(Cux.)mollis & 0 & 0 & 4 & 0 & 4 & 0,05 \\
\hline Cx.(Cux.)nignipalpus & 0 & 1 & 3 & 2 & 6 & 0,07 \\
\hline Cx. (Cux.)sp. & 1 & 12 & 29 & 84 & 126 & 1.54 \\
\hline Cx.(Mcx.)imitator & 0 & 0 & 2 & 0 & 2 & 0,02 \\
\hline Cx.(MCx.)pleunistriatus & 0 & 0 & 1 & 0 & 1 & 0,01 \\
\hline Cx.(Mel.)abonnenci & 0 & 0 & 1 & 0 & 1 & 0,01 \\
\hline Cx.(Mel.)bastagarius & 0 & 0 & 1 & 4 & 5 & 0.06 \\
\hline Cx.(Mel.)dunni & 0 & 0 & 3 & 2 & 5 & 0.06 \\
\hline Cx.(Mel.)evansae & 0 & 0 & 5 & 1 & 6 & 0.07 \\
\hline Cx.(Mel.)gahaoi & 0 & 0 & 13 & 10 & 23 & 0,28 \\
\hline Cx.(Mel.)gnupo Atratus & 0 & 1 & 0 & 0 & 1 & 0,01 \\
\hline Cx.(Mel.)gnupo Intrincatus & 0 & 5 & 4 & 1 & 10 & 0,12 \\
\hline Cx.(Mel.)gnipo Pilosus & 0 & 0 & 10 & 2 & 12 & 0,15 \\
\hline Cx.(Mel.)intrincatus & 0 & 0 & 8 & 0 & 8 & 0,1 \\
\hline Cx.(Mel.)oedipus & 0 & 1 & 1 & 1 & 3 & 0,04 \\
\hline Cx.(Mel.)pedroi & 0 & 0 & 2 & 0 & 2 & 0,02 \\
\hline Cx.(Mel.)plectoporpe & 0 & 0 & 0 & 1 & 1 & 0.01 \\
\hline Cx.(Mel.)rabelloi & 0 & 0 & 3 & 0 & 3 & 0,04 \\
\hline Cx.(Mel.)ribeirensis & 1 & 7 & 8 & 1 & 17 & 0,21 \\
\hline CX.(Mel.)sacchettae & 22 & 39 & 219 & 6 & 286 & 3,49 \\
\hline Cx.(Mel.)sp & 0 & 0 & 1 & 0 & 1 & 0,01 \\
\hline Cx.(Mel.)taeniopus & 0 & 2 & 14 & 0 & 16 & 0,2 \\
\hline Cx.(Mel.)theobaldi V. R. & 0 & 0 & 2 & 1 & 3 & 0,04 \\
\hline Li.durhami & 5 & 1 & 2 & 1 & 9 & 0,11 \\
\hline Liffavisetosus & 5 & 0 & 2 & 1 & 8 & 0,1 \\
\hline Ma.(Man.)indubitans & 0 & 0 & 2 & 0 & 2 & 0,02 \\
\hline Ma.(Man.)pseudotitillans & 1 & 0 & 0 & 0 & 1 & 0,01 \\
\hline Ma.(Man.)sp & 1 & 0 & 0 & 0 & 1 & 0.01 \\
\hline Ma.(Man.)titillans & 0 & 0 & 0 & 1 & 1 & 0.01 \\
\hline Ph. prox palmata/diabolica & 1 & 0 & 0 & 0 & 1 & 0,01 \\
\hline Ph.davisi & 1 & 0 & 0 & 0 & 1 & 0,01 \\
\hline Ph.palmata/diabolica & 0 & 0 & 1 & 0 & 1 & 0,01 \\
\hline
\end{tabular}


(cont.)

\begin{tabular}{lrrrrrr}
\hline Espócie & jan & fev & mar & abr & lotal & $F(\%)$ \\
\hline Ph.theobaldi & 1 & 4 & 7 & 2 & 14 & 0,17 \\
Ps.albigenu & 0 & 1 & 0 & 0 & 1 & 0,01 \\
Ps.albipes & 0 & 1 & 1 & 0 & 2 & 0,02 \\
Ps.ferox & 3 & 36 & 7 & 0 & 46 & 0,56 \\
Ru.reversa & 1 & 0 & 1 & 0 & 2 & 0,02 \\
Ur.calosomata & 0 & 0 & 5 & 4 & 9 & 0,11 \\
Ur.geometrica & 0 & 1 & 0 & 6 & 7 & 0,09 \\
Wy.felicia/pampeites & 0 & 0 & 0 & 1 & 1 & 0,01 \\
Wy.confusa & 0 & 0 & 6 & 6 & 12 & 0,15 \\
\hline & 2274 & 2443 & 2556 & 927 & 8200 & 100 \\
\hline
\end{tabular}

Tab.5: Número e frequencia relativa (F) de individuos coletados em armadilha tipo Shannon em área de mata primária (Sítio Gentil Cananéia) durante o período de junho de 1993 a abril de 1995.

\begin{tabular}{|c|c|c|c|c|c|}
\hline Espécie & 93 & 94 & 95 & Ntotal & $F(\%)$ \\
\hline Ae.argyrothorax & 2 & 2 & 5 & 9 & 0,034 \\
\hline Ae.crinifer & 0 & 1 & 0 & 1 & 0,004 \\
\hline Ae.gr.Serrat(hastoli) & 3 & 3 & 7 & 13 & 0,049 \\
\hline Ae.gr.Serrat(serrinub) & 18 & 38 & 15 & 71 & 0,267 \\
\hline Ae.hastatus & 0 & 1 & 0 & 1 & 0,004 \\
\hline Ae.oligopistus & 0 & 2 & 0 & 2 & 0,008 \\
\hline Ae.prox.perventor ou hortator & 0 & 2 & 2 & 4 & 0,015 \\
\hline Ae.scapularis & 77 & 114 & 454 & 645 & 2,426 \\
\hline Ae.sematus & 37 & 21 & 86 & 144 & 0,542 \\
\hline An.albitersis esp. $B$ & 0 & 1 & 0 & 1 & 0,004 \\
\hline An.(Ste.)kompi/canorii & 0 & 1 & 0 & 1 & 0,004 \\
\hline An.bellator & 1799 & 3198 & 2135 & 7132 & 26,82 \\
\hline An.cruzii & 2606 & 3021 & 4051 & 9678 & 36,4 \\
\hline An.intermedius & 0 & 0 & 1 & 1 & 0,004 \\
\hline An.prox.mediopunctatus & 1 & 1 & 1 & 3 & 0,011 \\
\hline Cq.chrysonotum & 25 & 89 & 55 & 169 & 0,636 \\
\hline Cq.chrysonotum ou albifera & 2376 & 3216 & 629 & 6221 & 23,4 \\
\hline Cq.hemanoi & 7 & 0 & 0 & 7 & 0,026 \\
\hline Cq.juxtemansonia & 248 & 73 & 0 & 321 & 1,207 \\
\hline Ca.nigricans & 2 & 1 & 0 & 3 & 0,011 \\
\hline Cq.venezuelensis & 79 & 195 & 72 & 346 & 1,301 \\
\hline Cx.(Cux.) gr Coronator & 10 & 30 & 20 & 60 & 0,226 \\
\hline Cx. (Cux.)bidens & 0 & 0 & 4 & 4 & 0,015 \\
\hline Cx.(Cux.)chidesteri & 0 & 1 & 2 & 3 & 0.011 \\
\hline Cx. $($ Cux.) dolosus & 1 & 0 & 0 & 1 & 0.004 \\
\hline Cx.(Cux.)mollis & 0 & 3 & 4 & 7 & 0,026 \\
\hline Cx.(Cux.)nigripalpus & 1 & 4 & 6 & 11 & 0,041 \\
\hline$C x .(C u x) s p$. & 32 & 108 & 126 & 266 & 1,0004 \\
\hline CX.(Lut)bigoti & 1 & 0 & 0 & 1 & 0,004 \\
\hline Cx.(Mcx.) lanei & 0 & 1 & 0 & 1 & 0,004 \\
\hline Cx.(Mcx.)grupo imitator & 0 & 1 & 0 & 1 & 0.004 \\
\hline
\end{tabular}




\begin{tabular}{|c|c|c|c|c|c|}
\hline Espécie & 93 & 94 & 95 & total & $\overline{F(\%)}$ \\
\hline CX.(Mcx.)imitator & 0 & 1 & 2 & 3 & 0,011 \\
\hline CX.(Mcx.).neglectus & 1 & 1 & 0 & 2 & 0,008 \\
\hline CX.(MCX.)pleuristriatus & 0 & 1 & 1 & 2 & 0,008 \\
\hline Cx.(Mel.)abonnenci & 0 & 0 & 1 & 1 & 0,004 \\
\hline Cx.(Mel.)bestagarius & 0 & 7 & 5 & 12 & 0,045 \\
\hline Cx.(Mel.)corentynensis & 2 & 0 & 0 & 2 & 0,008 \\
\hline CX.(Mel.)dunni & 0 & 2 & 5 & 7 & 0,026 \\
\hline Cx.(Mel.)evansae & 0 & 1 & 6 & 7 & 0,026 \\
\hline CX.(Mel.)gatvaoi & 3 & 8 & 23 & 34 & 0,128 \\
\hline Cx.(Mel.)grupo Atratus & 0 & 2 & 1 & 3 & 0,011 \\
\hline Cx.(Mel.)gnupo Intrincatus & 0 & 1 & 10 & 11 & 0,041 \\
\hline Cx.(Mel.)grupo Pilosus & 2 & 129 & 12 & 143 & 0,538 \\
\hline Cx.(Mel.)intrincatus & 0 & 0 & 8 & 8 & 0,03 \\
\hline CX.(Mel.)misionensis & 0 & 1 & 0 & 1 & 0,004 \\
\hline Cx.(Mel.)ocellatus & 6 & 0 & 0 & 6 & 0,023 \\
\hline Cx.(Mel.)ocossa & 2 & 2 & 0 & 4 & 0,015 \\
\hline Cx.(Mel.)oedipus & 0 & 0 & 3 & 3 & 0,011 \\
\hline Cx.(Mel.)pedroi & 1 & 1 & 2 & 4 & 0,015 \\
\hline Cx.(Mel.)plectoporpe & 0 & 6 & 1 & 7 & 0,026 \\
\hline CX.(Mel.)rabelloi & 0 & 2 & 3 & 5 & 0,019 \\
\hline Cx.(Mel.)ribeirensis & 41 & 35 & 17 & 93 & 0,35 \\
\hline CX.(Mel.)sacchettze & 31 & 107 & 286 & 424 & 1,595 \\
\hline CX.(Mel.)sp & 0 & 2 & 1 & 3 & 0,011 \\
\hline CX.(Mel.)treniopus & 0 & 2 & 16 & 18 & 0,068 \\
\hline$C_{x}$. Mel.)theobaldi V. $R$. & 0 & 9 & 3 & 12 & 0,045 \\
\hline Cx.(Mel.)zetekj & 1 & 2 & 0 & 3 & 0,011 \\
\hline Li.durhami & 14 & 55 & 9 & 78 & 0,293 \\
\hline Liflavisetosus & 2 & 8 & 8 & 18 & 0,068 \\
\hline Ma.(Man.)lindubitans & 0 & 3 & 2 & 5 & 0,019 \\
\hline Me.(Man.)pseudotitillans & 0 & 4 & 1 & 5 & 0,019 \\
\hline Ma.(Man.)sp & 3 & 14 & 1 & 18 & 0,068 \\
\hline Ma.(Man.)titillans & 0 & 0 & 1 & 1 & 0,004 \\
\hline Ph.prox.palmata/diabolica & 0 & 9 & 1 & 10 & 0,038 \\
\hline Ph.prox.theobaldi & 0 & 7 & 0 & 7 & 0,026 \\
\hline Ph.devisi & 0 & 0 & 1 & 1 & 0,004 \\
\hline Ph.fiabellata & 0 & 1 & 0 & 1 & 0,004 \\
\hline Ph.palmata/diabolica & 3 & 26 & 1 & 30 & 0,113 \\
\hline Ph.quasilongirostris & 17 & 32 & 0 & 49 & 0,184 \\
\hline Ph.theobeldi & 0 & 37 & 14 & 51 & 0,192 \\
\hline Phoniomyia sp & 0 & 1 & 0 & 1 & 0,004 \\
\hline Ps.albigenu & 5 & 26 & 1 & 32 & 0,12 \\
\hline Ps.albipes & 0 & 10 & 2 & 12 & 0,045 \\
\hline Ps.ferox & 5 & 7 & 46 & 58 & 0,218 \\
\hline Ru.humboldt & 0 & 1 & 0 & 1 & 0,004 \\
\hline Ru.prox.reversa & 0 & 1 & 0 & 1 & 0,004 \\
\hline Ru.reversa & 3 & 9 & 2 & 14 & 0,053 \\
\hline Ru.theobaldi & 0 & 1 & 0 & 1 & 0,004 \\
\hline Ur.calosomata & 6 & 4 & 9 & 19 & 0,071 \\
\hline Ur.geometrica & 15 & 19 & 7 & 41 & 0,154 \\
\hline Ur.lowii & 0 & 1 & 0 & 1 & 0,004 \\
\hline
\end{tabular}


(cont.)

\begin{tabular}{lrrrrr}
\hline Espécie & 93 & 94 & 95 & total & $F(\%)$ \\
\hline Ur.pulcherima & 6 & 0 & 0 & 6 & 0,023 \\
Wy.felicialpampeites & 1 & 25 & 1 & 27 & 0,102 \\
Wy.contuse & 34 & 106 & 12 & 152 & 0,572 \\
Wy.mystes ou finlayi & 1 & 0 & 0 & 1 & 0,004 \\
Wy.shannoni & 0 & 3 & 0 & 3 & 0,011 \\
\hline & 7530 & 10860 & 8200 & 26590 & 100 \\
\hline
\end{tabular}

Tab.6: Número e frequência relativa $(F)$ de indivíduos coletados em armadilha tipo Shannon em área de mata residual ("mata A" - Fazenda Experimental do IAC - Pariquera-Açu) durante o ano de 1992.

\begin{tabular}{|c|c|c|c|c|c|c|c|c|c|c|c|c|c|c|}
\hline Espécie & jan & fev & mar & $a b r$ & maio & jun & jul & ago & set & out & nov & dez & total & $F(\%)$ \\
\hline Ae.finiatilis & 0 & 0 & 0 & 0 & 0 & 0 & 0 & 0 & 7 & 0 & 0 & 0 & 7 & 0,06 \\
\hline Ae.fuhus & 0 & 2 & 13 & 3 & 0 & 1 & 0 & 0 & 0 & 0 & 0 & 0 & 19 & 0.16 \\
\hline Ae.gr.Serrat(hast/oli) & 0 & 0 & 0 & 0 & 393 & 0 & 0 & 0 & 2 & 0 & 0 & 0 & 395 & 3.29 \\
\hline Ae.gr.Serrat(serr/mub) & 8 & 11 & 242 & 69 & 54 & 71 & 7 & 7 & 7 & 6 & 16 & 31 & 529 & 44 \\
\hline Ae.scapularis & 35 & 19 & 934 & 19 & 180 & 16 & 17 & 7 & 0 & 9 & 18 & 215 & 1469 & 12.2 \\
\hline Ae.serratus & 16 & 40 & 623 & 197 & 30 & 45 & 25 & 17 & 10 & 13 & 21 & 219 & 1256 & 10,4 \\
\hline An albitarsis esp. A & 0 & 1 & 0 & 0 & 0 & 0 & 0 & 0 & 0 & 0 & 0 & 0 & 1 & 0.01 \\
\hline An. albitarsis & 6 & 0 & 0 & 0 & 0 & 0 & 0 & 0 & 0 & 0 & 0 & 0 & 6 & 0.05 \\
\hline An.cruzii & 0 & 2 & 1 & 0 & 0 & 0 & 0 & 0 & 0 & 0 & 12 & 56 & 71 & 0.59 \\
\hline An.grupo punctimacula & 0 & 0 & 1 & 0 & 0 & 0 & 0 & 2 & 0 & 0 & 0 & 0 & 3 & 0,02 \\
\hline An.intermedius & 0 & 0 & 2 & 1 & 0 & 1 & 0 & 0 & 0 & 0 & 0 & 5 & 9 & 0,07 \\
\hline An.oswaldoi & 1 & 1 & 0 & 0 & 0 & 0 & 0 & 0 & 0 & 0 & 0 & 0 & 2 & 0,02 \\
\hline An.prox.mediopunctatus & 1 & 1 & 4 & 5 & 15 & 2 & 3 & 1 & 0 & 1 & 3 & 17 & 53 & 0,44 \\
\hline An.strodei & 0 & 0 & 0 & 0 & 0 & 0 & 1 & 0 & 0 & 0 & 0 & 0 & 1 & 0.01 \\
\hline An.triannulatus & 0 & 0 & 2 & 0 & 0 & 0 & 0 & 0 & 0 & 0 & 0 & 0 & 2 & 0.02 \\
\hline Cq.albicosta & 1 & 1 & 0 & 0 & 0 & 0 & 0 & 0 & 0 & 0 & 0 & 0 & 2 & 0.02 \\
\hline Cq.chrysonotum & 1 & 10 & 28 & 0 & 0 & 0 & 0 & 0 & 0 & 0 & 4 & 13 & 56 & 0,47 \\
\hline Cq. chrysonotumvalbifere & 41 & 92 & 404 & 33 & 24 & 34 & 0 & 0 & 0 & 7 & 131 & 218 & 984 & 8.18 \\
\hline Cq.hermanoi & 11 & 1 & 5 & 1 & 0 & 2 & 0 & 0 & 0 & 0 & 16 & 0 & 36 & 0,3 \\
\hline Cq.juxtamansonia & 12 & 6 & 0 & 2 & 0 & 2 & 0 & 0 & 0 & 0 & 21 & 5 & 48 & 0.4 \\
\hline Cq.nigricans & 0 & 0 & 1 & 0 & 0 & 0 & 0 & 0 & 0 & 0 & 2 & 0 & 3 & 0.02 \\
\hline Cq.venezuelensis & 50 & 20 & 25 & 2 & 24 & 8 & 3 & 1 & 4 & 16 & 269 & 47 & 469 & 3.9 \\
\hline Cx.(Aed.)amazonensis & 0 & 0 & 2 & 1 & 0 & 0 & 0 & 0 & 0 & 0 & 0 & 0 & 3 & 0.02 \\
\hline Cx.(Cux.) gr Coronator & 8 & 1 & 22 & 1 & 21 & 0 & 3 & 0 & 6 & 0 & 0 & 6 & 68 & 0.57 \\
\hline Cx.(Cux.)chidester & 0 & 1 & 2 & 0 & 0 & 0 & 0 & 0 & 0 & 0 & 0 & 0 & 3 & 0.02 \\
\hline Cx. (Cux.)comiger & 0 & 0 & 5 & 0 & 0 & 0 & 0 & 0 & 0 & 0 & 0 & 0 & 5 & 0.04 \\
\hline Cx. (Cux.)coronator & 0 & 0 & 0 & 0 & 0 & 0 & 0 & 4 & 0 & 0 & 0 & 1 & 5 & 0.04 \\
\hline Cx.(Cux.)declarator & 0 & 0 & 6 & 0 & 0 & 0 & 0 & 0 & 0 & 0 & 0 & 0 & 6 & 0.05 \\
\hline Cx.(Cux.)dolosus/eduardoi & 0 & 0 & 0 & 0 & 0 & 0 & 0 & 2 & 0 & 0 & 0 & 3 & 5 & 0.04 \\
\hline Cx.(Cux.)lygrus & 0 & 0 & 0 & 0 & 1 & 0 & 0 & 1 & 0 & 0 & 0 & 2 & 4 & 0.03 \\
\hline Cx.(Cux.)mollis & 0 & 0 & 3 & 0 & 0 & 0 & 0 & 0 & 0 & 0 & 0 & 0 & 3 & 0.02 \\
\hline Cx.(Cux.)nigripalpus & 2 & 388 & 1593 & 54 & 120 & 0 & 2 & 2 & 0 & 7 & 22 & & 2193 & 18.2 \\
\hline Cx. (Cux.)sp. & 6 & 18 & 25 & 1 & 15 & 0 & 2 & 0 & 0 & 3 & 0 & 0 & 70 & 0.58 \\
\hline Cx.(Lut)bigoti & 0 & 0 & 0 & 0 & 0 & 0 & 0 & 0 & 0 & 1 & 0 & 0 & $i$ & 0.01 \\
\hline Cx.(Mel.)aureonotatus & 3 & 17 & 0 & 0 & 0 & 0 & 0 & 0 & 0 & 0 & 0 & 0 & 20 & 0.17 \\
\hline CX.(Mel.)bastagarius & 59 & 31 & 16 & 2 & 0 & 0 & 0 & 0 & 0 & 0 & 0 & 3 & 111 & 0.92 \\
\hline Cx.(Mel.)gnupo Atratus & 1 & 2 & 2 & 1 & 0 & 0 & 3 & 1 & 0 & 0 & 0 & 3 & 13 & 0,11 \\
\hline
\end{tabular}


(cont.)

\begin{tabular}{|c|c|c|c|c|c|c|c|c|c|c|c|c|c|}
\hline Espécie & jan & fev & mar & $a b r$ & maio & jun & jul & ago & set & out & nov & $\operatorname{dez}$ & total $F(\%)$ \\
\hline Cx.(Mel.)grupo Pilosus & 2 & 58 & 49 & 0 & 0 & 0 & 0 & 0 & 0 & 0 & 0 & 0 & 1090,91 \\
\hline Cx.(Mel.)innovator & 0 & 0 & 2 & 0 & 0 & 0 & 0 & 0 & 0 & 0 & 0 & 0 & 20,02 \\
\hline Cx.(Mel.)ocossa & 4 & 0 & 3 & 0 & 0 & 0 & 0 & 0 & 3 & 2 & 0 & 0 & 120,1 \\
\hline Cx.(Mel.)oedipus & 0 & 0 & 1 & 2 & 0 & 0 & 0 & 0 & 0 & 0 & 0 & 15 & 180,15 \\
\hline Cx.(Mel.)pedroi & 0 & 0 & 2 & 1 & 0 & 0 & 0 & 0 & 0 & 0 & 0 & 0 & 30,02 \\
\hline Cx.(Mel.)pereyrai & 0 & 0 & 3 & 1 & 0 & 0 & 0 & 0 & 0 & 0 & 0 & 1 & 50,04 \\
\hline CX.(Mel.)plectoporpe & 0 & 0 & 0 & 0 & 0 & 0 & 0 & 0 & 1 & 0 & 0 & 3 & 40,03 \\
\hline Cx.(Mel.)ribeirensis & 85 & 75 & 612 & 70 & 75 & 0 & 52 & 46 & 45 & 21 & 37 & 136 & 125410,4 \\
\hline Cx.(Mel.)sacchettee & 2 & 6 & 21 & 17 & 12 & 0 & 4 & 0 & 0 & 0 & 26 & 75 & 1631,36 \\
\hline Cx.(Mel.)sp & 0 & 1 & 3 & 1 & 0 & 0 & 0 & 0 & 0 & 0 & 0 & 1 & 60,05 \\
\hline Cx.(Mel.)sp sec Melanoc. & 0 & 0 & 0 & 0 & 0 & 0 & 0 & 0 & 2 & 0 & 0 & 0 & 20,02 \\
\hline Cx.(Mel.)spissipes & 0 & 0 & 6 & 3 & 0 & 0 & 0 & 0 & 0 & 0 & 0 & 0 & 90,07 \\
\hline Cx.(Mel.)taeniopus & 0 & 0 & 0 & 1 & 0 & 0 & 0 & 0 & 0 & 0 & 0 & 0 & 10,01 \\
\hline Cx. (Mel.)theobaldi V.R. & 45 & 68 & 113 & 0 & 0 & 0 & 0 & 0 & 0 & 0 & 0 & 0 & 2261,88 \\
\hline Cx.(Mel.)zeteki & 0 & 0 & 3 & 3 & 0 & 0 & 0 & 1 & 0 & 0 & 0 & 3 & $10 \quad 0,08$ \\
\hline Lidurhami & 3 & 5 & 0 & 6 & 0 & 3 & 10 & 0 & 7 & 3 & 0 & 6 & 430,36 \\
\hline Lifflavisetosus & 8 & 3 & 1 & 5 & 0 & 2 & 0 & 0 & 0 & 2 & 0 & 0 & 210,17 \\
\hline Ma.(Man.)humeralis & 0 & 0 & 0 & 0 & 0 & 0 & 0 & 0 & 0 & 0 & 12 & 0 & 120,1 \\
\hline Ma.(Man.)indubitans & 4 & 51 & 90 & 7 & 9 & 13 & 19 & 0 & 2 & 0 & 63 & 18 & $276 \quad 2,3$ \\
\hline Ma.(Man.)sp & 21 & 20 & 15 & 5 & 6 & 6 & 15 & 3 & 0 & 0 & 36 & 9 & 1361,13 \\
\hline Ph.prox.flabellata & 1 & 0 & 0 & 0 & 0 & 0 & 0 & 0 & 0 & 0 & 0 & 0 & 10,01 \\
\hline Ph.davisi & 0 & 0 & 0 & 0 & 0 & 0 & 0 & 1 & 1 & 0 & 0 & 1 & 30,02 \\
\hline Ph.quasilongirostris & 2 & 1 & 0 & 0 & 0 & 0 & 0 & 0 & 0 & 0 & 6 & 0 & 90,07 \\
\hline Ph.theobaldi & 0 & 0 & 0 & 0 & 0 & 0 & 3 & 0 & 0 & 0 & 2 & 0 & 50,04 \\
\hline Ps.albigenu & 6 & 0 & 260 & 2 & 6 & 1 & 0 & 0 & 0 & 0 & 0 & 936 & 121110,1 \\
\hline Ps.albigenurvaripes & 0 & 0 & 12 & 0 & 0 & 0 & 0 & 0 & 0 & 0 & 0 & 350 & 3623,01 \\
\hline Ps.albipes & 0 & 0 & 6 & 0 & 0 & 0 & 0 & 0 & 0 & 0 & 0 & 0 & 60,05 \\
\hline Ps.discrucians & 0 & 0 & 0 & 0 & 0 & 0 & 0 & 0 & 0 & 0 & 0 & 1 & 10,01 \\
\hline Ps.ferox & 0 & 0 & 81 & 3 & 6 & 2 & 0 & 0 & 0 & 1 & 2 & 8 & 1030,86 \\
\hline Ps.lutzii & 0 & 0 & 8 & 0 & 0 & 0 & 0 & 0 & 0 & 0 & 0 & 4 & 120,1 \\
\hline Ru.humboldt & 0 & 1 & 1 & 0 & 0 & 0 & 0 & 0 & 0 & 0 & 0 & 0 & 20,02 \\
\hline Ru.reverse & 0 & 0 & 0 & 0 & 0 & 0 & 0 & 0 & 0 & 2 & 0 & 1 & 30,02 \\
\hline Ru.theobaldi & 0 & 0 & 0 & 1 & 0 & 0 & 0 & 0 & 0 & 0 & 0 & 0 & 10,01 \\
\hline Tr.digitatum & 0 & 0 & 0 & 0 & 0 & 0 & 0 & 0 & 0 & 0 & 1 & 0 & 10,01 \\
\hline Ur.calosomata & 0 & 0 & 0 & 0 & 0 & 0 & 0 & 1 & 0 & 0 & 0 & 1 & 20,02 \\
\hline Ur.lowii & 0 & 5 & 9 & 0 & 0 & 0 & 0 & 0 & 0 & 0 & 0 & 0 & 140,12 \\
\hline Ur.mathesoni & 0 & 0 & 1 & 0 & 0 & 0 & 0 & 0 & 0 & 0 & 0 & 0 & 10,01 \\
\hline Wy.felicio/pampeites & 0 & 0 & 0 & 0 & 0 & 0 & 0 & 0 & 0 & 0 & 0 & 1 & 10,01 \\
\hline Wy. eirosaihowa/luteove & 0 & 0 & 0 & 0 & 0 & 0 & 1 & 0 & 0 & 0 & 0 & 0 & 10.01 \\
\hline Wy.aporonoma & 1 & 1 & 3 & 1 & 0 & 0 & 0 & 0 & 0 & 0 & 0 & 0 & 60.05 \\
\hline Wy.confusa & 2 & 1 & 0 & 4 & 3 & 0 & 1 & 3 & 7 & 6 & 6 & 2 & 350,29 \\
\hline
\end{tabular}


Tab.8: Número e frequência relativa $(F)$ de indivíduos coletados em armadilha tipo Shannon em área de mata residual ("mata A" - Fazenda Experimental do IAC Pariquera-Açu) durante o ano de 1993

\begin{tabular}{|c|c|c|c|c|c|c|c|c|c|c|c|c|c|c|}
\hline Espécie & jan & fev & mar & $a b r$ & maio & jun & jui & ago & set & out & nov & dez & total & $F(\%)$ \\
\hline Ad.squamipennis & 0 & 3 & 0 & 0 & 0 & 0 & 0 & 0 & 0 & 0 & 0 & 0 & 3 & 0,03 \\
\hline Ae, albopictus & 0 & 0 & 0 & 0 & 0 & 0 & 0 & 0 & 0 & 0 & 1 & 0 & 1 & 0,01 \\
\hline Ae.crinifer & 0 & 0 & 0 & 0 & 0 & 0 & 1 & 0 & 0 & 2 & 0 & 0 & 3 & 0,03 \\
\hline Ae.fulvus & 2 & 28 & 49 & 2 & 0 & 0 & 0 & 0 & 0 & 2 & 4 & 0 & 87 & 0,74 \\
\hline Ae.gr.Serrat(hast/oli) & 0 & 0 & 11 & 0 & 0 & 0 & 0 & 0 & 2 & 8 & 0 & 6 & 27 & 0,23 \\
\hline Ae.gr.Sertat(serr/nub) & 0 & 245 & 265 & 28 & 36 & 4 & 14 & 15 & 22 & 44 & 119 & 54 & 846 & 7,16 \\
\hline Ae.nubilus & 16 & 0 & 0 & 0 & 0 & 0 & 0 & 0 & 0 & 0 & 0 & 0 & 16 & 0,14 \\
\hline Ae.scapularis & 0 & 42 & 61 & 11 & 25 & 15 & 42 & 18 & 5 & 60 & 0 & 2 & 281 & 2,38 \\
\hline Ae.serratus & 25 & 508 & 382 & 140 & 109 & 19 & 123 & 79 & 20 & 73 & 285 & 298 & 2061 & 17,4 \\
\hline An.albitarsis esp. B & 0 & 43 & 31 & 2 & 0 & 0 & 0 & 1 & 0 & 0 & 0 & 0 & 77 & 0,65 \\
\hline An.(Nys.)sec.Argyr/Alb & 0 & 1 & 0 & 0 & 0 & 0 & 0 & 0 & 0 & 0 & 0 & 0 & 1 & 0,01 \\
\hline An albitarsis & 0 & 0 & 0 & 0 & 0 & 0 & 1 & 0 & 0 & 0 & 0 & 0 & 1 & 0,01 \\
\hline An.cruzij & 0 & 4 & 12 & 4 & 0 & 0 & 0 & 1 & 0 & 14 & 0 & 1 & 36 & 0,3 \\
\hline An evansee & 0 & 0 & 1 & 0 & 2 & 0 & 0 & 0 & 0 & 0 & 0 & 0 & 3 & 0,03 \\
\hline An.gelvaoi & 0 & 0 & 0 & 0 & 0 & 0 & 0 & 1 & 0 & 0 & 0 & 0 & 1 & 0,01 \\
\hline An.gnupo punctimacula & 0 & 0 & 8 & 0 & 0 & 0 & 0 & 1 & 0 & 0 & 0 & 0 & 9 & 0,08 \\
\hline An.intermedius & 0 & 4 & 22 & 0 & 0 & 0 & 2 & 0 & 0 & 0 & 0 & 0 & 28 & 0,24 \\
\hline An.prox.mediopunctatus & 0 & 2 & 27 & 7 & 2 & 0 & 3 & 0 & 0 & 1 & 0 & 0 & 42 & 0.36 \\
\hline An.strodei & 1 & 0 & 0 & 0 & 0 & 0 & 0 & 1 & 0 & 0 & 0 & 0 & 2 & 0,02 \\
\hline An.triannulatus & 0 & 2 & 0 & 0 & 0 & 0 & 0 & 0 & 0 & 0 & 0 & 0 & 2 & 0,02 \\
\hline Cq.albicosta & 0 & 2 & 0 & 4 & 0 & 0 & 0 & 0 & 0 & 0 & 7 & 0 & 13 & 0,11 \\
\hline Cq.chrysonotum & 0 & 19 & 10 & 0 & 0 & 0 & 0 & 0 & 0 & 0 & 0 & 0 & 29 & 0,25 \\
\hline Cq.chnysonotumvalbifera & 3 & 407 & 344 & 864 & 24 & 2 & 0 & 0 & 1 & 49 & 238 & 41 & 1973 & 16,7 \\
\hline Cq.hermanoi & 3 & 18 & 7 & 2 & 0 & 0 & 0 & 0 & 0 & 0 & 16 & 6 & 52 & 0,44 \\
\hline Cq.juxtemansonia & 12 & 2 & 16 & 2 & 3 & 1 & 0 & 0 & 1 & 1 & 0 & 16 & 54 & 0,46 \\
\hline Cq.nignicans & 7 & 0 & 6 & 0 & 0 & 0 & 0 & 0 & 0 & 0 & 8 & 0 & 21 & 0.18 \\
\hline Cq.venezuelensis & 0 & 44 & 114 & 78 & 46 & 10 & 0 & 2 & 3 & 12 & 71 & 31 & 411 & 3,48 \\
\hline Cx.(Aed).amazonensis & 0 & 26 & 16 & 11 & 4 & 0 & 1 & 0 & 0 & 0 & 0 & 1 & 59 & 0.5 \\
\hline Cx, $($ Cux.) gr Coronator & 0 & 3 & 2 & 7 & 1 & 1 & 6 & 12 & 13 & 9 & 3 & 0 & 57 & 0,48 \\
\hline Cx.(Cux.)bidens & 0 & 0 & 0 & 1 & 0 & 0 & 1 & 0 & 0 & 2 & 0 & 0 & 4 & 0.03 \\
\hline Cx.(Cux.)chidesteri & 0 & 3 & 0 & 0 & 0 & 0 & 0 & 1 & 0 & 5 & 0 & 0 & 9 & 0.08 \\
\hline Cx.(Cux.)comiger & 0 & 0 & 0 & 0 & 0 & 0 & 0 & 0 & 1 & 0 & 0 & 0 & 1 & 0,01 \\
\hline CX. $(\mathrm{Cux}$.)coronator & 0 & 0 & 0 & 0 & 0 & 0 & 0 & 0 & 2 & 0 & 0 & 0 & 2 & 0.02 \\
\hline CX.(Cux,) declarator & 0 & 1 & 0 & 0 & 0 & 0 & 0 & 0 & 0 & 0 & 0 & 0 & 1 & 0,01 \\
\hline Cx, $($ Cux.)dolosus & 0 & 0 & 0 & 0 & 0 & 0 & 0 & 1 & 0 & 0 & 0 & 0 & 1 & 0,01 \\
\hline Cx.(Cux.)dol/eduardoi & 0 & 2 & 0 & 0 & 0 & 0 & 0 & 0 & 0 & 0 & 0 & 0 & 2 & 0,02 \\
\hline Cx.(Cux.)mollis & 0 & 3 & 2 & 0 & 0 & 0 & 0 & 0 & 0 & 0 & 0 & 0 & 5 & 0,04 \\
\hline Cx.(Cux.)nigripalpus & 4 & 63 & 148 & 12 & 0 & 1 & 2 & 0 & 2 & 0 & 0 & 2 & 234 & 1,98 \\
\hline Cx.(Cux.)sp. & 0 & 12 & 3 & 3 & 1 & 0 & 2 & 3 & 0 & 0 & 0 & 0 & 24 & 0.2 \\
\hline Cx.(Mcx.)grupo imitator & 0 & 0 & 4 & 0 & 0 & 0 & 0 & 0 & 0 & 0 & 0 & 0 & 4 & 0,03 \\
\hline Cx.(Mcx.)imitator & 0 & 0 & 1 & 0 & 0 & 0 & 0 & 0 & 0 & 0 & 0 & 0 & 1 & 0.01 \\
\hline Cx.(Mcx.)pleuristriatus & 0 & 0 & 0 & 2 & 0 & 0 & 0 & 0 & 0 & 0 & 0 & 0 & 2 & 0,02 \\
\hline Cx.(Mel.)aureonotatus & 0 & 14 & 7 & 0 & 0 & 0 & 0 & 0 & 0 & 0 & 0 & 0 & 21 & 0.18 \\
\hline Cx.(Mel.)bastagarius & 0 & 30 & 39 & 12 & 0 & 1 & 2 & 1 & 0 & 1 & 0 & 0 & 86 & 0.73 \\
\hline CX.(Mel.)deipontei & 0 & 0 & 1 & 0 & 0 & 0 & 0 & 0 & 0 & 0 & 0 & 0 & 1 & 0.01 \\
\hline Cx.(Mel.)distinguendus & 0 & 0 & 2 & 0 & 0 & 0 & 0 & 0 & 0 & 0 & 0 & 0 & 2 & 0,02 \\
\hline Cx.(Mel.)dunni & 0 & 1 & 2 & 1 & 1 & 0 & 0 & 0 & 0 & 1 & 0 & 0 & 6 & 0,05 \\
\hline CX.(Mel.)eastor & 0 & 0 & 1 & 0 & 0 & 0 & 0 & 0 & 0 & 0 & 0 & 0 & 1 & 0.01 \\
\hline
\end{tabular}




\begin{tabular}{|c|c|c|c|c|c|c|c|c|c|c|c|c|c|c|}
\hline Espécie & jan & fev & mar & abr $n$ & 1aio & jun & jul & ago & set & out & nov & dez & total & $F(\%)$ \\
\hline CX.(Mel) levansae & 0 & 0 & 6 & 0 & 1 & 0 & 0 & 0 & 0 & 0 & 0 & 0 & 7 & 0,06 \\
\hline CX.(Mel.)faurani & 0 & 0 & 2 & 8 & 4 & 0 & 0 & 0 & 0 & 0 & 0 & 0 & 14 & 0,12 \\
\hline Cx.(Mel.)grupo Atratus & 6 & 4 & 5 & 3 & 2 & 0 & 1 & 1 & 0 & 0 & 0 & 0 & 22 & 0,19 \\
\hline Cx.(Mel.)gr intrincatus & 0 & 1 & 0 & 0 & 0 & 0 & 0 & 0 & 0 & 0 & 0 & 0 & 1 & 0,01 \\
\hline Cx.(Mel.)grupo Pilosus & 0 & 23 & 19 & 2 & 1 & 0 & 0 & 1 & 0 & 2 & 1 & 0 & 49 & 0,41 \\
\hline Cx.(Mel.)inadmirabilis & 0 & 0 & 0 & 0 & 0 & 0 & 0 & 0 & 0 & 1 & 0 & 0 & 1 & 0,01 \\
\hline Cx.(Mel.)innovator & 0 & 0 & 0 & 0 & 0 & 0 & 1 & 0 & 0 & 0 & 0 & 0 & 1 & 0,01 \\
\hline CX.(Mel.)intrincetus & 0 & 1 & 0 & 0 & 0 & 0 & 0 & 0 & 0 & 0 & 0 & 0 & 1 & 0,01 \\
\hline Cx.(Mel.)lopesi & 0 & 1 & 0 & 0 & 0 & 0 & 0 & 0 & 0 & 0 & 0 & 0 & 1 & 0,01 \\
\hline Cx.(Mel.)misionensis & 0 & 1 & 0 & 0 & 1 & 0 & 0 & 0 & 1 & 0 & 0 & 0 & 3 & 0,03 \\
\hline Cx.(Mel.)ocossa & 0 & 0 & 20 & 0 & 0 & 0 & 0 & 0 & 0 & 0 & 0 & 3 & 23 & 0,19 \\
\hline Cx.(Mel.)oedipus & 0 & 4 & 1 & 5 & 0 & 0 & 0 & 0 & 0 & 2 & 0 & 0 & 12 & 0,1 \\
\hline Cx.(Mel.)pedroi & 0 & 9 & 3 & 10 & 0 & 0 & 0 & 0 & 0 & 6 & 0 & 0 & 28 & 0,24 \\
\hline Cx.(Mel.)pereyraj & 0 & 1 & 0 & 0 & 0 & 0 & 0 & 0 & 0 & 0 & 0 & 0 & 1 & 0,01 \\
\hline Cx.(Mel.)pilosus & 0 & 0 & 0 & 15 & 0 & 0 & 0 & 0 & 0 & 0 & 0 & 0 & 15 & 0,13 \\
\hline CX.(Mel.)plectoporpe & 1 & 2 & 0 & 0 & 0 & 0 & 0 & 0 & 0 & 1 & 0 & 0 & 4 & 0,03 \\
\hline Cx.(Mel.)rabelloi & 0 & 1 & 0 & 0 & 0 & 0 & 0 & 0 & 0 & 0 & 0 & 0 & 1 & 0,01 \\
\hline Cx. (Mel) )ribeirensis & 15 & 700 & 331 & 149 & 66 & 9 & 68 & 19 & 53 & 22 & 52 & 15 & 1499 & 12,7 \\
\hline CX.(Mel.)sacchettae & 28 & 102 & 267 & 614 & 99 & 0 & 11 & 7 & 14 & 10 & 13 & 7 & 1172 & 9,92 \\
\hline Cx.(Mel.)sp & 0 & 0 & 3 & 0 & 0 & 0 & 0 & 0 & 0 & 0 & 0 & 0 & 3 & 0,03 \\
\hline Cx. (Mel.)sp secMelanoc. & 0 & 2 & 0 & 0 & 0 & 0 & 0 & 0 & 0 & 0 & 0 & 0 & 2 & 0,02 \\
\hline Cx.(Mel.)spissipes & 0 & 10 & 4 & 0 & 0 & 0 & 0 & 0 & 0 & 0 & 0 & 0 & 14 & 0,12 \\
\hline CX.(Mel.)taeniopus & 0 & 9 & 2 & 4 & 3 & 0 & 0 & 0 & 1 & 0 & 0 & 0 & 19 & 0,16 \\
\hline Cx.(Mel.)theobaldi V. R. & 0 & 25 & 12 & 1 & 0 & 0 & 0 & 0 & 0 & 0 & 0 & 0 & 38 & 0,32 \\
\hline Cx.(Mel.)zeteki & 0 & 1 & 0 & 2 & 0 & 0 & 3 & 2 & 0 & 0 & 0 & 0 & 8 & 0,07 \\
\hline Li.durhemi & 1 & 60 & 79 & 30 & 15 & 3 & 11 & 9 & 2 & 2 & 0 & 16 & 228 & 1,93 \\
\hline Liffavisetosus & 0 & 13 & 46 & 4 & 6 & 3 & 3 & 1 & 1 & 0 & 0 & 3 & 80 & 0,68 \\
\hline Ma.(Men.)fleveola & 0 & 0 & 0 & 0 & 0 & 0 & 1 & 0 & 0 & 0 & 0 & 0 & 1 & 0,01 \\
\hline Ma.(Man.)indubitans & 6 & 81 & 7 & 31 & 15 & 6 & 15 & 13 & 9 & 12 & 2 & 0 & 137 & 1,67 \\
\hline Ma.(Man.)pseudotitillans & 0 & 5 & 0 & 0 & 0 & 0 & 0 & 0 & 0 & 0 & 0 & 0 & 5 & 0,04 \\
\hline Ma.(Man.)sp & 3 & 102 & 55 & 0 & 0 & 4 & 9 & 2 & 14 & 0 & 0 & 0 & 189 & 1,6 \\
\hline Ma.(Man.)titillans & 1 & 0 & 0 & 0 & 0 & 0 & 0 & 2 & 0 & 0 & 0 & 0 & 3 & 0,03 \\
\hline Ph. prox. palm/diabolica & 3 & 0 & 0 & 0 & 0 & 0 & 0 & 3 & 0 & 0 & 0 & 0 & 6 & 0,05 \\
\hline Ph.palmata/diabolica & 0 & 0 & 7 & 0 & 0 & 0 & 0 & 0 & 0 & 0 & 0 & 0 & 7 & 0,06 \\
\hline Ph.quasilongirostris & 11 & 2 & 0 & 0 & 0 & 1 & 4 & 0 & 0 & 2 & 0 & 0 & 20 & 0,17 \\
\hline Ph.theobaldi & 0 & 0 & 1 & 0 & 0 & 0 & 0 & 0 & 0 & 0 & 0 & 0 & 1 & 0,01 \\
\hline Ps. albigenu & 0 & 73 & 225 & 105 & 1 & 1 & 0 & 0 & 0 & 100 & 0 & 0 & 505 & 4,27 \\
\hline Ps. albigenurvaripes & 0 & 57 & 229 & 0 & 0 & 1 & 2 & 0 & 0 & 2 & 0 & 0 & 291 & 2,46 \\
\hline Ps.albipes & 0 & 0 & 0 & 0 & 0 & 0 & 0 & 0 & 0 & 225 & 0 & 0 & 225 & 1,9 \\
\hline Ps.cingulata & 0 & 0 & 0 & 0 & 0 & 0 & 1 & 0 & 0 & 0 & 0 & 0 & 1 & 0,01 \\
\hline Ps.discnucians & 0 & 23 & 0 & 0 & 0 & 0 & 0 & 0 & 0 & 1 & 0 & 0 & 24 & 0,2 \\
\hline Ps.ferox & 7 & 42 & 96 & 3 & 3 & 0 & 12 & 0 & 0 & 88 & 2 & 9 & 262 & 2,22 \\
\hline Ps.lutzii & 0 & 8 & 12 & 0 & 0 & 0 & 1 & 0 & 0 & 3 & 0 & 0 & 24 & 0,2 \\
\hline Ru. prox. reversa & 0 & 0 & 3 & 0 & 0 & 0 & 0 & 0 & 0 & 0 & 0 & 0 & 3 & 0,03 \\
\hline Ru.reversa & 0 & 2 & 9 & 1 & 0 & 0 & 0 & 0 & 0 & 0 & 0 & 1 & 13 & 0,11 \\
\hline Tr.digitatum & 1 & 0 & 0 & 0 & 0 & 0 & 0 & 0 & 0 & 0 & 0 & 3 & 4 & 0,03 \\
\hline Ur.apicalis & 0 & 1 & 0 & 1 & 0 & 0 & 0 & 0 & 0 & 0 & 0 & 0 & 2 & 0,02 \\
\hline Ur.calosomata & 0 & 0 & 2 & 0 & 1 & 0 & 1 & 0 & 1 & 0 & 0 & 0 & 5 & 0,04 \\
\hline Ur.geometrica & 0 & 0 & 1 & 0 & 1 & 0 & 0 & 0 & 0 & 0 & 0 & 3 & 5 & 0,04 \\
\hline Ur.lowii & 0 & 1 & 0 & 1 & 0 & 0 & 0 & 0 & 0 & 0 & 0 & 2 & 4 & 0,03 \\
\hline
\end{tabular}




\begin{tabular}{lrrrrrrrrrrrrrrrr} 
(cont.) & jan & fev & mar & abr maio & jun & jul & ago & set & out & nov & dez & total & $F(\%)$ \\
\hline Especie & 0 & 1 & 0 & 0 & 0 & 0 & 1 & 0 & 0 & 0 & 0 & 0 & 2 & 0,02 \\
\hline Ur.mathesoni & 0 & 0 & 0 & 0 & 0 & 0 & 1 & 0 & 0 & 0 & 0 & 0 & 1 & 0,01 \\
Ur.nataliae & 0 & 1 & 0 & 0 & 0 & 0 & 0 & 0 & 0 & 0 & 0 & 0 & 1 & 0,01 \\
Ur.pulchemima & 1 & 0 & 3 & 0 & 0 & 0 & 0 & 0 & 0 & 0 & 2 & 2 & 8 & 0,07 \\
Wy.felicia/pampeites & 0 & 0 & 1 & 2 & 1 & 0 & 0 & 0 & 0 & 0 & 0 & 0 & 4 & 0,03 \\
Wy.airosaihowa/ut. & 0 & 1 & 5 & 0 & 0 & 0 & 0 & 0 & 0 & 6 & 0 & 0 & 12 & 0.1 \\
Wy.aporonoma & 5 & 46 & 41 & 13 & 1 & 0 & 7 & 5 & 2 & 0 & 3 & 16 & 139 & 1,18 \\
Wy.confusa & 0 & 0 & 1 & 0 & 0 & 1 & 0 & 1 & 0 & 0 & 0 & 0 & 3 & 0,03 \\
Wy.mystes ou finlayi & 162 & 2948 & 3092 & 2197 & 475 & 83 & 353 & 203 & 170 & 769 & 827 & 538 & 11817 & 100
\end{tabular}

Tab.9: Número e frequência relativa $(F)$ de individuos coletados em armadilha tipo Shannon em área de mata residual ("mata A" - Fazenda Experimental do $1 \mathrm{AC}$ - Pariquera-Açu) durante o ano de 1994.

\begin{tabular}{|c|c|c|c|c|c|c|c|c|c|c|c|c|c|c|}
\hline Espécie & jan & fev & mar & $a b r$ & maio & jun & jul & ago & set & out & nov & dez & total & $F(\%)$ \\
\hline Ae.crinifer & 0 & 1 & 0 & 0 & 0 & 0 & 0 & 0 & 0 & 0 & 0 & 0 & 1 & 0,02 \\
\hline Ae.fulvus & 0 & 7 & 41 & 1 & 0 & 0 & 0 & 0 & 0 & 0 & 0 & 0 & 49 & 0.97 \\
\hline Ae.gr.Serrat(sentrnub) & 96 & 24 & 51 & 23 & 21 & 5 & 4 & 1 & 0 & 0 & 3 & 0 & 228 & 4.49 \\
\hline Ae.nubilus & 0 & 0 & 43 & 0 & 0 & 0 & 0 & 0 & 0 & 0 & 0 & 0 & 43 & 0.85 \\
\hline Ae.scapularis & 28 & 26 & 24 & 61 & 15 & 34 & 21 & 55 & 0 & 2 & 10 & 6 & 282 & 5.56 \\
\hline Ae.serratus & 232 & 112 & 162 & 103 & 29 & 13 & 5 & 5 & 0 & 0 & 21 & 12 & 694 & 13.7 \\
\hline An.albitarsis esp. B & 1 & 1 & 0 & 0 & 0 & 0 & 0 & 2 & 0 & 0 & 0 & 0 & 4 & 0,08 \\
\hline An.cruzï & 4 & 0 & 3 & 1 & 0 & 0 & 0 & 1 & 1 & 1 & 4 & 2 & 17 & 0.33 \\
\hline An.intermedius & 0 & 0 & 0 & 6 & 0 & 0 & 0 & 0 & 0 & 0 & 0 & 0 & 6 & 0,12 \\
\hline An.oswaldoi & 0 & 0 & 0 & 0 & 0 & 1 & 0 & 0 & 0 & 0 & 1 & 0 & 2 & 0.04 \\
\hline An.prox.mediopu & 0 & 0 & 7 & 1 & 0 & 0 & 0 & 1 & 0 & 2 & 0 & r & 12 & 0.24 \\
\hline An.triannulatus & 0 & 2 & 1 & 0 & 0 & 0 & 0 & 0 & 0 & 0 & 0 & 0 & 3 & 0.06 \\
\hline Cq.albicosta & 0 & 1 & 0 & 3 & 0 & 0 & 0 & 0 & 0 & 0 & 0 & 0 & 4 & 0,08 \\
\hline Cq.chrysonotum & 0 & 1 & 0 & 0 & 0 & 0 & 0 & 0 & 0 & 3 & 0 & 0 & 4 & 0.08 \\
\hline Cq. chrysonotuma & 36 & 120 & 110 & 465 & 4 & 1 & 0 & 0 & 2 & 54 & 72 & 29 & 893 & 17.5 \\
\hline Cq.hermanoi & 5 & 11 & 2 & 2 & 1 & 0 & 0 & 0 & 0 & 2 & 0 & 1 & 24 & 0.47 \\
\hline Cq.juxtamansonia & 14 & 10 & 13 & 24 & 0 & 0 & 0 & 0 & 0 & 3 & 3 & 0 & 67 & 1,32 \\
\hline Cq.venezuelensis & 19 & 60 & 20 & 20 & 5 & 13 & 1 & 1 & 4 & 30 & 10 & 4 & 187 & 3.68 \\
\hline CX.(Aed.)amazonen & 0 & 4 & 6 & 2 & 0 & 0 & 0 & 1 & 0 & 1 & 3 & & 17 & 0.33 \\
\hline Cx.(Cux.) gr Coronator & 0 & 3 & 0 & 1 & 0 & 2 & 9 & 9 & 3 & 0 & 0 & 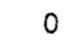 & 27 & 0.53 \\
\hline Cx. (Cux.)chidesteri & 0 & 0 & 0 & 0 & 0 & 3 & 0 & 0 & 3 & 0 & 0 & 0 & 6 & 0,12 \\
\hline Cx.(Cux.)lygnus & 0 & 0 & 0 & 0 & 0 & 0 & 0 & 0 & 0 & 1 & 0 & 0 & 1 & 0.02 \\
\hline Cx.(Cux.)mollis & 0 & 0 & 0 & 0 & 0 & 0 & 0 & 1 & 2 & 0 & 1 & 0 & 4 & 0,08 \\
\hline Cx.(Cux.)nigrip & 0 & 0 & 7 & 1 & 0 & 1 & 0 & 0 & 0 & 1 & 0 & 3 & 13 & 0.25 \\
\hline$C x .(C u x) s p$, & 0 & 0 & 2 & 1 & 0 & 3 & 1 & 4 & 1 & 0 & 2 & & 15 & 0.3 \\
\hline CX.(Mel.)bastage & 0 & 7 & 3 & 11 & 0 & 2 & 0 & 0 & 0 & 9 & 0 & & 32 & 0.63 \\
\hline Cx.(Mel.)dunni & 0 & 0 & 0 & 2 & 4 & 1 & 0 & 0 & 1 & 0 & 0 & 0 & 8 & 0.16 \\
\hline Cx.(Mel.)eastor & 0 & 0 & 0 & 1 & 0 & 1 & 0 & 0 & 0 & 0 & 0 & 0 & 2 & 0.04 \\
\hline Cx, (Mel).ensiformis & 1 & 0 & 0 & 0 & 0 & 0 & 0 & 0 & 0 & 0 & 0 & 0 & 1 & 0.02 \\
\hline Cx.(Mel.)evansae & 0 & 0 & 0 & 2 & 2 & 1 & 0 & 0 & 0 & 0 & 0 & 0 & 5 & 01 \\
\hline Cx.(Mel.)faurani & 0 & 0 & 0 & 1 & 0 & 0 & 0 & 0 & 0 & 0 & 0 & 0 & 1 & 0,02 \\
\hline Cx.(Mel.)grupo Atratus & 0 & 0 & 0 & 1 & 2 & 0 & 0 & 0 & 0 & 0 & 1 & 1 & 5 & 0.1 \\
\hline CX.(Mel.)gr. Intrincatus & 0 & 0 & 0 & 1 & 0 & 1 & 0 & 0 & 1 & 1 & 0 & 0 & 4 & 0,08 \\
\hline
\end{tabular}


(cont.)

\begin{tabular}{|c|c|c|c|c|c|c|c|c|c|c|c|c|c|c|}
\hline Espécie & jan & fov & $\operatorname{mar}$ & $a b r$ & maio & jun & jul & ago & set & out & nov & $d \theta z$ & total & $F(\%)$ \\
\hline CX.(Mel.)grupo Pilosus & 0 & 0 & 5 & 5 & 0 & 0 & 0 & 0 & 1 & 5 & 0 & 2 & 18 & 0,35 \\
\hline CX.(Mel.)innovetor & 0 & 0 & 0 & 0 & 0 & 0 & 0 & 0 & 0 & 0 & 1 & 0 & 1 & 0,02 \\
\hline Cx.(Mel.)ocossa & 6 & 0 & 0 & 0 & 0 & 3 & 0 & 0 & 0 & 1 & 0 & 0 & 10 & 0,2 \\
\hline Cx.(Mel.)oedipus & 0 & 0 & 0 & 0 & 0 & 2 & 0 & 0 & 0 & 0 & 1 & 0 & 3 & 0,06 \\
\hline CX.(Mel.)pedroi & 1 & 0 & 0 & 0 & 0 & 0 & 0 & 1 & 1 & 0 & 1 & 0 & 4 & 0,08 \\
\hline Cx.(Mel.)pilosus & 0 & 1 & 5 & 0 & 0 & 0 & 0 & 0 & 0 & 0 & 0 & 0 & 6 & 0,12 \\
\hline Cx.(Mel.)plectoporpe & 0 & 1 & 1 & 0 & 1 & 2 & 2 & 0 & 0 & 3 & 0 & 0 & 10 & 0,2 \\
\hline Cx.(Mel.)ribeirensis & 21 & 100 & 99 & 44 & 28 & 31 & 13 & 104 & 2 & 25 & 32 & 94 & 593 & 11,7 \\
\hline CX.(Mel.)sacchettae & 5 & 1 & 6 & 32 & 27 & 10 & 16 & 34 & 29 & 34 & 10 & 15 & 219 & 4,32 \\
\hline CX. (Mel.)sp & 0 & 0 & 0 & 0 & 0 & 0 & 0 & 0 & 0 & 3 & 0 & 1 & 4 & 0,08 \\
\hline Cx.(Mel.)spissipes & 0 & 0 & 1 & 0 & 0 & 0 & 0 & 0 & 2 & 0 & 0 & 1 & 4 & 0,08 \\
\hline Cx.(Mel.)taeniopus & 0 & 0 & 0 & 2 & 0 & 0 & 0 & 0 & 0 & 0 & 0 & 1 & 3 & 0,06 \\
\hline Cx. (Mel.)theobaldi V. $R$. & 1 & 5 & 2 & 6 & 0 & 6 & 0 & 0 & 0 & 10 & 0 & 2 & 32 & 0,63 \\
\hline Cx.(Mel.)zeteki & 0 & 0 & 0 & 2 & 0 & 0 & 0 & 0 & 0 & 0 & 0 & 0 & 2 & 0.04 \\
\hline Lidurhami & 42 & 13 & 90 & 8 & 3 & 3 & 4 & 6 & 0 & 1 & 24 & 35 & 229 & 4,51 \\
\hline Liffavisetosus & 8 & 6 & 2 & 0 & 1 & 0 & 2 & 8 & 0 & 1 & 3 & 0 & 31 & 0,61 \\
\hline Ma.(Man.)humeralis & 0 & 0 & 0 & 2 & 0 & 0 & 0 & 1 & 0 & 0 & $\uparrow$ & 0 & 4 & 0,08 \\
\hline Ma.(Man.)indubitans & 5 & 18 & 36 & 98 & 4 & 10 & 7 & 61 & 8 & 26 & 4 & 2 & 279 & 5.5 \\
\hline Ma.(Man.)pseudotitillans & 0 & 1 & 0 & 1 & 0 & $\uparrow$ & 0 & 0 & 0 & 0 & 0 & 0 & 3 & 0,06 \\
\hline Ma.(Man.)sp & 5 & 6 & 0 & 10 & 3 & 10 & 9 & 66 & 6 & 9 & 4 & 5 & 133 & 2,62 \\
\hline Ma.(Men.)titillans & 0 & 1 & 0 & 0 & 0 & 0 & 0 & 0 & 0 & 0 & 0 & 0 & 1 & 0,02 \\
\hline Ph.prox. theobaldi & 0 & 0 & 1 & 0 & 0 & 0 & 0 & 0 & 0 & 0 & 0 & 0 & 1 & 0,02 \\
\hline Ph.davisi & 0 & 0 & 0 & 0 & 0 & 0 & 0 & 0 & 0 & 0 & 0 & 1 & 1 & 0,02 \\
\hline Ph.palmata/diabolica & 0 & 0 & 0 & 0 & 0 & 0 & 0 & 0 & 0 & 0 & 0 & 1 & 1 & 0,02 \\
\hline Ph.quasilongirostris & 9 & 17 & 2 & 0 & 1 & 0 & 0 & 0 & 0 & 0 & 1 & 0 & 30 & 0.59 \\
\hline Ph.theobaldi & 0 & 0 & 6 & 0 & 0 & 2 & 0 & 0 & 0 & 1 & 3 & 0 & 12 & 0.24 \\
\hline Ps.albigenu & 39 & 165 & 111 & 3 & 0 & 0 & 0 & 2 & 0 & 0 & 0 & 0 & 320 & 6,31 \\
\hline Ps.albigenurvaripes & 2 & 13 & 0 & 1 & 0 & 0 & 0 & 0 & 0 & 0 & 0 & 0 & 16 & 0,32 \\
\hline Ps.albipes & 8 & 0 & 0 & 0 & 0 & 0 & 0 & 0 & 0 & 0 & 0 & 0 & 8 & 0,16 \\
\hline Ps.discrucians & 0 & 1 & 0 & 0 & 0 & 0 & 0 & 0 & 0 & 0 & 0 & 0 & 1 & 0,02 \\
\hline Ps.ferox & 97 & 24 & 53 & 3 & 0 & 0 & 0 & 0 & 0 & 0 & 1 & 3 & 181 & 3.57 \\
\hline$P_{S . / u t z i j}$ & 0 & 2 & 0 & 0 & 0 & 0 & 0 & 0 & 0 & 0 & 0 & 0 & 2 & 0,04 \\
\hline Ru.reversa & 0 & 2 & 13 & 0 & 0 & 1 & 0 & 2 & 1 & 1 & 0 & 6 & 26 & 0.51 \\
\hline Ru.theobaldi & 0 & 0 & 1 & 1 & 0 & 0 & 0 & 0 & 0 & 0 & 0 & 0 & 2 & 0.04 \\
\hline Sa.chloroptenus & 1 & 0 & 0 & 0 & 0 & 0 & 0 & 0 & 0 & 0 & 0 & 0 & 1 & 0,02 \\
\hline Tr.digitatum & 2 & 1 & 0 & 0 & 0 & 0 & 0 & 1 & 0 & 0 & 0 & 1 & 5 & 0.1 \\
\hline Ur.calosomata & 0 & 0 & 0 & 1 & 0 & 0 & 2 & 0 & 0 & 1 & 0 & 0 & 4 & 0,08 \\
\hline Ur.geometrica & 0 & 1 & 0 & 0 & 0 & 0 & 0 & 0 & 2 & 0 & 0 & 0 & 3 & 0,06 \\
\hline Ur.lowii & 0 & 0 & 0 & 1 & 0 & 0 & 0 & 0 & 0 & 0 & 0 & 0 & 1 & 0.02 \\
\hline Ur.mathesoni & 0 & 0 & 0 & 0 & 0 & 2 & 0 & 2 & 0 & 0 & 0 & 0 & 4 & 0.08 \\
\hline Ur.pulchemima & 0 & 1 & 0 & 0 & 0 & 0 & 0 & 0 & 0 & 2 & 0 & 0 & 3 & 0.06 \\
\hline Wy.felicia'pampeites & 0 & 0 & 5 & 0 & 0 & 6 & 0 & 1 & 0 & 2 & 0 & 0 & 14 & 0,28 \\
\hline Wy.eporonoma & 1 & 0 & 0 & 0 & 0 & 2 & 0 & 1 & 0 & 0 & 0 & 0 & 4 & 0.08 \\
\hline Wy.contuse & 8 & 13 & 0 & 6 & 1 & 4 & 4 & 11 & 0 & 5 & 15 & 4 & 71 & 14 \\
\hline Wy.mystes ou finlayi & 0 & 0 & 111 & 0 & 0 & 0 & 0 & 0 & 0 & 0 & 0 & 0 & 111 & 2,19 \\
\hline Wy.shannoni & 0 & 0 & 2 & 0 & 0 & 0 & 0 & 1 & 0 & 0 & 0 & 0 & 3 & 0,06 \\
\hline
\end{tabular}


Tab.10: Número e frequencia relativa $(F)$ de indivíduos coletados em armadilha tipo Shannon em área de mata residual ("mata A" - Fazenda Experimental do LAC - Pariquera-Açu) de janeiro de 1992 a dezembro de 1994.

\begin{tabular}{|c|c|c|c|c|c|}
\hline Espécie & 92 & 93 & 94 & Ntotal & $F(\%)$ \\
\hline Ad.squemipennis & 0 & 3 & 0 & 3 & 0,01 \\
\hline Ae. albopictus & 0 & 1 & 0 & 1 & 0,003 \\
\hline Ae.crinifer & 0 & 3 & 1 & 4 & 0,014 \\
\hline Ae.ficuatilis & 7 & 0 & 0 & 7 & 0,024 \\
\hline Ae.fulvus & 19 & 87 & 49 & 155 & 0,536 \\
\hline Ae.gr.Serrat(hast/oli) & 395 & 27 & 0 & 422 & 1,459 \\
\hline Ae.gr.Serrat(sern/mub) & 529 & 846 & 228 & 1603 & 5,544 \\
\hline Ae.nubilus & 0 & 16 & 43 & 59 & 0,204 \\
\hline Ae scopularis & 1469 & 281 & 282 & 2032 & 7,027 \\
\hline Ae.serratus & 1256 & 2061 & 694 & 4011 & 13,87 \\
\hline An.albitarsis esp. A & 1 & 0 & 0 & 1 & 0,003 \\
\hline An albitarsis esp. B & 0 & 77 & 4 & 81 & 0,28 \\
\hline An.(Nys.) seces Argyrit ou Albim & 0 & 1 & 0 & 1 & 0,003 \\
\hline An.elbitarsis & 6 & 1 & 0 & 7 & 0,024 \\
\hline An.cruzii & 71 & 36 & 17 & 124 & 0,429 \\
\hline An.evansae & 0 & 3 & 0 & 3 & 0,01 \\
\hline An.galvaoi & 0 & 1 & 0 & 1 & 0,003 \\
\hline An.gnupo punctimacula & 3 & 9 & 0 & 12 & 0,042 \\
\hline An.intermedius & 9 & 28 & 6 & 43 & 0,149 \\
\hline An.oswaldoi & 2 & 0 & 2 & 4 & 0,014 \\
\hline An.prox.mediopunctatus & 53 & 42 & 12 & 107 & 0,37 \\
\hline An.strodei & 1 & 2 & 0 & 3 & 0,01 \\
\hline An.triannulatus & 2 & 2 & 3 & 7 & 0,024 \\
\hline Cq.elbicoste & 2 & 13 & 4 & 19 & 0,066 \\
\hline Cq.chrysonotum & 56 & 29 & 4 & 89 & 0,308 \\
\hline Cq.chrysonotum ou albifera & 984 & 1973 & 893 & 3850 & 13,31 \\
\hline Cq.hemanoi & 36 & 52 & 24 & 112 & 0,387 \\
\hline Cq.juxtamansonia & 48 & 54 & 67 & 169 & 0,584 \\
\hline Cq.nigricans & 3 & 21 & 0 & 24 & 0,083 \\
\hline Cq.venezuelensis & 469 & 411 & 187 & 1067 & 3,69 \\
\hline Cx.(Aed.)amezonensis & 3 & 59 & 17 & 79 & 0.273 \\
\hline Cx.(Cux.) gr Coronator & 68 & 57 & 27 & 152 & 0,526 \\
\hline Cx.(Cux.)bidens & 0 & 4 & 0 & 4 & 0,014 \\
\hline Cx.(Cux.)chidesteri & 3 & 9 & 6 & 18 & 0,062 \\
\hline Cx.(Cux.)comiger & 5 & 1 & 0 & 6 & 0,021 \\
\hline Cx.(Cux.)coronator & 5 & 2 & 0 & 7 & 0,024 \\
\hline Cx.(Cux.)declarator & 6 & 1 & 0 & 7 & 0,024 \\
\hline Cx.(Cux.)dolosus & 0 & 1 & 0 & 1 & 0,003 \\
\hline Cx.(Cux.)dolosus/eduardoi & 5 & 2 & 0 & 7 & 0,024 \\
\hline Cx.(Cux.)lygrus & 4 & 0 & 1 & 5 & 0,017 \\
\hline Cx.(Cux.)mollis & 3 & 5 & 4 & 12 & 0.042 \\
\hline Cx.(Cux.)nigripalpus & 2193 & 234 & 13 & 2440 & 8,439 \\
\hline$C_{x}($ Cux.)sp. & 70 & 24 & 15 & 109 & 0,377 \\
\hline Cx.(Lut)bigoti & 1 & 0 & 0 & 1 & 0,003 \\
\hline Cx.(Mcx.)grupo imitator & 0 & 4 & 0 & 4 & 0,016 \\
\hline Cx.(Mcx.)imitator & 0 & 1 & 0 & 1 & 0,003 \\
\hline CX.(Mcx.)pleuristriatus & 0 & 2 & 0 & 2 & 0,007 \\
\hline Cx.(Mel.)aureonotatus & 20 & 21 & 0 & 41 & 0,142 \\
\hline
\end{tabular}




\begin{tabular}{|c|c|c|c|c|c|}
\hline Espécie & 92 & 93 & 94 & Ntotal & $F(\%)$ \\
\hline CX.(Mel.)bastegarius & 111 & 86 & 32 & 229 & 0,792 \\
\hline Cx.(Mel.)delpontei & 0 & 1 & 0 & 1 & 0,003 \\
\hline CX.(Mel.)distinguendus & 0 & 2 & 0 & 2 & 0,007 \\
\hline Cx.(Nel.)dunni & 0 & 6 & 8 & 14 & 0,048 \\
\hline Cx.(Mal.)eastor & 0 & 1 & 2 & 3 & 0,01 \\
\hline CX.(Mel.)ensiformis & 0 & 0 & 1 & 1 & 0,003 \\
\hline Cx.(Mol).evansae & 0 & 7 & 5 & 12 & 0,042 \\
\hline Cx.(Mel.)faurani & 0 & 14 & 1 & 15 & 0,052 \\
\hline Cx.(Mel.)grupo Atratus & 13 & 22 & 5 & 40 & 0,138 \\
\hline CX.(Mel.)grupo Intrincatus & 0 & 1 & 4 & 5 & 0,017 \\
\hline CX.(Mel.)gnupo Pilosus & 109 & 49 & 18 & 176 & 0,609 \\
\hline Cx.(Mel.)inedmirabilis & 0 & 1 & 0 & 1 & 0,003 \\
\hline Cx.(Mel.)innovator & 2 & 1 & 1 & 4 & 0,014 \\
\hline CX.(Mel.)intrincekus & 0 & 1 & 0 & 1 & 0,003 \\
\hline Cx.(Mel.)lopesi & 0 & 1 & 0 & 1 & 0,003 \\
\hline Cx.(Mel.)misionensis & 0 & 3 & 0 & 3 & 0,01 \\
\hline Cx.(Mel.)ocossa & 12 & 23 & 10 & 45 & 0,156 \\
\hline Cx.(Mel.)oedipus & 18 & 12 & 3 & 33 & 0,114 \\
\hline Cx.(Mel.)pedroi & 3 & 28 & 4 & 35 & 0,121 \\
\hline Cx.(Mel.)pereyrai & 5 & 1 & 0 & 6 & 0,021 \\
\hline Cx.(Mel.)pilosus & 0 & 15 & 6 & 21 & 0,073 \\
\hline Cx.(Mel.)plectoporpe & 4 & 4 & 10 & 18 & 0,062 \\
\hline Cx.(Mel.)rabelloi & 0 & 1 & 0 & 1 & 0,003 \\
\hline Cx.(Mel.)ribeirensis & 1254 & 1499 & 593 & 3346 & 11,57 \\
\hline CX. (Mel.)sacchettae & 163 & 1172 & 219 & 1554 & 5,374 \\
\hline Cx. $(\mathrm{Mel})$ spp & 6 & 3 & 4 & 13 & 0,045 \\
\hline Cx. (Mel.)sp sec Melenoc. & 2 & 2 & 0 & 4 & 0,014 \\
\hline Cx. (Mel.)spissipes & 9 & 14 & 4 & 27 & 0,093 \\
\hline Cx.(Mel.)taeniopus & 1 & 19 & 3 & 23 & 0,08 \\
\hline Cx. (Mel.)theobaldi V. $R$. & 226 & 38 & 32 & 296 & 1.024 \\
\hline Cx.(Mel.)zeteki & 10 & 8 & 2 & 20 & 0.069 \\
\hline Lidurhami & 43 & 228 & 229 & 500 & 1,729 \\
\hline Liffenisetosus & 21 & 80 & 31 & 132 & 0.457 \\
\hline Ma.(Man.)fleveola & 0 & 1 & 0 & 1 & 0,003 \\
\hline Ma.(Man).humeralis & 12 & 0 & 4 & 16 & 0.055 \\
\hline Ma. (Man.).indubitans & 276 & 197 & 279 & 752 & 2,601 \\
\hline Ma.(Man.)pseudotidillans & 0 & 5 & 3 & 8 & 0.028 \\
\hline Ma. (Man.)sp & 136 & 189 & 133 & 458 & 1.584 \\
\hline Ma.(Man.)titillans & 0 & 3 & 1 & 4 & 0,014 \\
\hline Ph. Prox. flabellata & 1 & 0 & 0 & 1 & 0,003 \\
\hline Ph. Prox palmata/diabolica & 0 & 6 & 0 & 6 & 0.021 \\
\hline Ph. Prox, theobaldi & 0 & 0 & 1 & 1 & 0,003 \\
\hline Ph.davisi & 3 & 0 & 1 & 4 & 0.014 \\
\hline Ph.palmata/diabolica & 0 & 7 & 1 & 8 & 0.028 \\
\hline Ph.quasilongirostris & 9 & 20 & 30 & 59 & 0.204 \\
\hline Ph.theobaldi & 5 & 1 & 12 & 18 & 0.062 \\
\hline Ps.albigenu & 1211 & 505 & 320 & 2036 & 7.041 \\
\hline Ps.albigenuvaripes & 362 & 291 & 16 & 669 & 2,314 \\
\hline Ps.albipes & 6 & 225 & 8 & 239 & 0.827 \\
\hline Ps.cingulate & 0 & 1 & 0 & 1 & 0,003 \\
\hline
\end{tabular}


(cont.)

\begin{tabular}{|c|c|c|c|c|c|}
\hline Espécie & 92 & 93 & 94 & Ntotal & $F(\%)$ \\
\hline Ps.discrucians & 1 & 24 & 1 & 26 & 0,09 \\
\hline Ps.ferox & 103 & 262 & 181 & 546 & 1,888 \\
\hline Ps.lutzii & 12 & 24 & 2 & 38 & 0,131 \\
\hline Ru.humbolat & 2 & 0 & 0 & 2 & 0,007 \\
\hline Ru.prox. reversa & 0 & 3 & 0 & 3 & 0,01 \\
\hline Ru.reverse & 3 & 13 & 26 & 42 & 0,145 \\
\hline Ru.theobaldi & 1 & 0 & 2 & 3 & 0,01 \\
\hline Sa.chloropterus & 0 & 0 & 1 & 1 & 0,003 \\
\hline Tr.digitetum & 1 & 4 & 5 & 10 & 0,035 \\
\hline Ur.apicalis & 0 & 2 & 0 & 2 & 0,007 \\
\hline Ur.calosomata & 2 & 5 & 4 & 11 & 0,038 \\
\hline Ur.geometrice & 0 & 5 & 3 & 8 & 0,028 \\
\hline Ur.lowii & 14 & 4 & 1 & 19 & 0,066 \\
\hline Ur.mathesoni & 1 & 2 & 4 & 7 & 0,024 \\
\hline Ur.nataliae & 0 & 1 & 0 & 1 & 0,003 \\
\hline Ur.pulcherrima & 0 & 1 & 3 & 4 & 0,014 \\
\hline Wy.felicia/pampeites & 1 & 8 & 14 & 23 & 0,08 \\
\hline Wy. eirosaihowa/uteove & 1 & 4 & 0 & 5 & 0,017 \\
\hline Wy.eporonoma & 6 & 12 & 4 & 22 & 0,076 \\
\hline Wy.confuse & 35 & 139 & 71 & 245 & 0,847 \\
\hline Wy.mystes ou finlayi & 0 & 3 & 111 & 114 & 0,394 \\
\hline \multirow[t]{2}{*}{ Wy.shannoni } & 0 & 0 & 3 & 3 & 0,01 \\
\hline & 12023 & 11817 & 5075 & 28915 & 100 \\
\hline
\end{tabular}

Tab.11: Número e frequência relativa $(\mathrm{F})$ de individuos coletados em armadilha tipo Shannon em área de mata residual ("mata B" - Fazenda Experimental do IAC Pariquera-Açu) durante o ano de 1992.

\begin{tabular}{lrrrrrrrrrrrrrrrr}
\hline Espécie & fev & mar & abr & maio & jun & jul & ago & set & out & nov & dez & total & $F(\%)$ \\
\hline Ae.crinifer & 0 & 0 & 0 & 0 & 0 & 0 & 0 & 2 & 0 & 0 & 0 & 2 & 0,05 \\
Ae.fumus & 1 & 1 & 5 & 28 & 0 & 0 & 0 & 0 & 0 & 0 & 3 & 38 & 0.92 \\
Ae.gr.Serrat(hastroli) & 0 & 0 & 2 & 0 & 0 & 0 & 0 & 0 & 0 & 0 & 0 & 2 & 0,05 \\
Ae.gr.Serrat(sern/mub) & 15 & 46 & 68 & 54 & 53 & 2 & 12 & 60 & 10 & 21 & 56 & 397 & 9,58 \\
Ae.oligopistus & 0 & 0 & 2 & 0 & 0 & 0 & 0 & 0 & 0 & 0 & 0 & 2 & 0,05 \\
Ae.scapularis & 6 & 41 & 53 & 45 & 18 & 0 & 2 & 18 & 93 & 0 & 46 & 322 & 7,77 \\
Ae.serratus & 13 & 162 & 77 & 140 & 133 & 27 & 16 & 92 & 115 & 62 & 90 & 927 & 22,4 \\
An.cruzii & 1 & 2 & 0 & 0 & 0 & 0 & 0 & 3 & 0 & 19 & 0 & 25 & 0,6 \\
An.grupo punctimacula & 0 & 0 & 0 & 0 & 0 & 0 & 0 & 1 & 0 & 0 & 0 & 1 & 0,02 \\
An.intermedius & 0 & 0 & 0 & 0 & 0 & 0 & 0 & 0 & 2 & 0 & 0 & 2 & 0,05 \\
An.oswaldoi & 0 & 0 & 0 & 0 & 0 & 0 & 0 & 0 & 1 & 0 & 0 & 1 & 0,02 \\
An.prox.mediopunctatus & 0 & 9 & 1 & 9 & 9 & 1 & 0 & 5 & 5 & 5 & 5 & 49 & 1,18 \\
Cq.chrysonotum & 0 & 0 & 0 & 0 & 0 & 0 & 0 & 0 & 1 & 1 & 0 & 2 & 0,05 \\
Cq.chrysonotumalbifera & 11 & 79 & 9 & 18 & 2 & 0 & 0 & 7 & 226 & 280 & 56 & 688 & 16,6 \\
Cq.hermanoi & 1 & 0 & 0 & 0 & 0 & 0 & 0 & 0 & 0 & 1 & 0 & 2 & 0,05 \\
Cq.venezuelensis & 8 & 3 & 3 & 9 & 2 & 0 & 0 & 0 & 47 & 26 & 51 & 149 & 3,6 \\
Cx.(Aed.)amazonensis & 0 & 0 & 1 & 0 & 0 & 0 & 0 & 0 & 5 & 0 & 0 & 6 & 0,14 \\
Cx.(Cux.) gr Coronator & 0 & 0 & 3 & 6 & 1 & 0 & 0 & 7 & 6 & 19 & 0 & 42 & 1,01 \\
Cx.(Cux.)chidesteri & 1 & 0 & 0 & 0 & 0 & 0 & 0 & 0 & 0 & 0 & 0 & 1 & 0,02 \\
Cx.(Cux.)declarator & 0 & 1 & 1 & 0 & 0 & 0 & 0 & 0 & 0 & 0 & 0 & 2 & 0,05 \\
Cx.(Cux.)dolosus/eduardoi & 0 & 0 & 0 & 0 & 0 & 0 & 2 & 2 & 0 & 0 & 0 & 4 & 0,1 \\
\hline
\end{tabular}


(cont.)

\begin{tabular}{|c|c|c|c|c|c|c|c|c|c|c|c|c|c|}
\hline Espócie & fev & mar & $a b r$ & maio & jun & jul & ago & set & out & nov & dez & total & $(F \%)$ \\
\hline Cx.(Cux.)mollis & 0 & 0 & 5 & 0 & 0 & 0 & 0 & 0 & 0 & 0 & 0 & 5 & 0,12 \\
\hline Cx.(Cux.)nigripalpus & 1 & 23 & 27 & 7 & 1 & 0 & 0 & 0 & 5 & 2 & 0 & 66 & 1,59 \\
\hline Cx. (Cux.)sp. & 1 & 4 & 2 & 1 & 1 & 0 & 0 & 0 & 0 & 0 & 0 & 9 & 0,22 \\
\hline CX. (Lut.)bigoti & 0 & 0 & 0 & 0 & 0 & 0 & 0 & 1 & 0 & 0 & 0 & 1 & 0,02 \\
\hline Cx.(MCx.)grupo imitator & 0 & 0 & 0 & 0 & 0 & 0 & 0 & 0 & 39 & 0 & 0 & 39 & 0,94 \\
\hline CX.(Mel.)oliciae & 0 & 0 & 0 & 0 & 0 & 0 & 0 & 0 & 1 & 0 & 0 & 1 & 0,02 \\
\hline Cx.(Mel.)aureonotatus & 0 & 0 & 1 & 0 & 0 & 0 & 0 & 0 & 0 & 0 & 0 & 1 & 0,02 \\
\hline Cx.(Mel.)bastegerius & 8 & 0 & 1 & 0 & 0 & 0 & 0 & 0 & 0 & 0 & 0 & 9 & 0,22 \\
\hline$C_{x}($ Mel, $)$ dunni & 0 & 0 & 3 & 0 & 0 & 0 & 0 & 0 & 0 & 0 & 0 & 3 & 0,07 \\
\hline CX.(Mel.)evensae & 0 & 0 & 0 & 0 & 0 & 0 & 0 & 0 & 0 & 1 & 0 & 1 & 0,02 \\
\hline Cx.(Mel.)galvaoi & 0 & 0 & 0 & 0 & 0 & 0 & 0 & 0 & 2 & 0 & 0 & 2 & 0,05 \\
\hline Cx.(Mel.)grupo Atratus & 15 & 0 & 8 & 8 & 7 & 0 & 2 & 3 & 0 & 4 & 7 & 54 & 1,3 \\
\hline Cx.(Mel.)grupo Pilosus & 0 & 9 & 1 & 0 & 0 & 0 & 0 & 0 & 2 & 0 & 0 & 12 & 0,29 \\
\hline Cx.(Mel.)innovator & 0 & 0 & 3 & 0 & 0 & 0 & 0 & 0 & 3 & 0 & 0 & s & 0,14 \\
\hline Cx.(Mel.)misionensis & 0 & 0 & 2 & 0 & 0 & 0 & 0 & 1 & 0 & 145 & 0 & 148 & 3,57 \\
\hline Cx.(Mel.)oedipus & 0 & 0 & 1 & 0 & 0 & 0 & 0 & 1 & 13 & 0 & 0 & 15 & 0,36 \\
\hline CX.(Mel.)pedroi & 0 & 0 & 9 & 0 & 0 & 0 & 0 & 0 & 0 & 0 & 0 & 9 & 0,22 \\
\hline Cx.(Mel.)pilosus & 0 & 0 & 2 & 0 & 0 & 0 & 0 & 0 & 6 & 0 & 0 & 8 & 0,19 \\
\hline Cx.(Mel.)plectoporpe & 0 & 0 & 0 & 0 & 0 & 0 & 0 & 0 & 4 & 0 & 0 & 4 & 0,1 \\
\hline Cx.(Mel.)ribeirensis & 7 & 25 & 23 & 30 & 13 & 2 & 16 & 103 & 38 & 0 & 33 & 290 & 7 \\
\hline CX.(Mel.)sacchettae & 5 & 2 & 3 & 17 & 7 & 0 & 1 & 22 & 10 & 59 & 39 & 165 & 3,98 \\
\hline$C_{x}(\mathrm{Mel})$ sp & 0 & 0 & 0 & 1 & 0 & 0 & 0 & 0 & 0 & 0 & 0 & 1 & 0,02 \\
\hline Cx.(Mel.)spissipes & 0 & 5 & 6 & 4 & 0 & 0 & 0 & 0 & 0 & 2 & 0 & 17 & 0,41 \\
\hline Cx.(Mel.)taeniopus & 0 & 0 & 2 & 0 & 0 & 0 & 0 & 0 & 0 & 1 & 2 & 5 & 0,12 \\
\hline Cx.(Mel.)theobaldi V.R. & 0 & 0 & 0 & 0 & 0 & 0 & 0 & 0 & 0 & 15 & 0 & 15 & 0,36 \\
\hline Cx.(Mel.)zeteki & 0 & 0 & 0 & 0 & 0 & 0 & 0 & 0 & 0 & 1 & 0 & 1 & 0,02 \\
\hline Li.durhami & 10 & 2 & 2 & 2 & 1 & 0 & 6 & 11 & 15 & 1 & 27 & 77 & 1,86 \\
\hline Liffavisetosus & 4 & 0 & 0 & 2 & 1 & 0 & 2 & 8 & 7 & 1 & 0 & 25 & 0,6 \\
\hline Ma. (Man.)indubitans & 0 & 1 & 2 & 1 & 17 & 0 & 0 & 0 & 4 & 4 & 41 & 70 & 1,69 \\
\hline Ma.(Man.)sp & 0 & 0 & 0 & 0 & 4 & 0 & 0 & 2 & 0 & 0 & 0 & 6 & 0,14 \\
\hline Ph. Prox. flabellata & 0 & 0 & 0 & 0 & 0 & 0 & 1 & 0 & 0 & 0 & 0 & 1 & 0,02 \\
\hline Ph.pelmate/diabolica & 0 & 0 & 0 & 0 & 0 & 0 & 0 & 0 & 0 & 0 & 7 & 7 & 0,17 \\
\hline Ph.quasitongirostris & 1 & 1 & 0 & 0 & 1 & 0 & 0 & 0 & 0 & 0 & 3 & 6 & 0,14 \\
\hline Ps.albigenu & 0 & 61 & 4 & 2 & 0 & 0 & 0 & 0 & 4 & 0 & 20 & 91 & 2.2 \\
\hline Ps.albigenurvaripes & 0 & 30 & 0 & 0 & 0 & 0 & 0 & 0 & 0 & 0 & 0 & 30 & 0,72 \\
\hline Ps.discrucians & 0 & 4 & 0 & 0 & 0 & 0 & 0 & 0 & 0 & 0 & 0 & 4 & 0,1 \\
\hline Ps.ferox & 1 & 11 & 6 & 3 & 0 & 0 & 0 & 1 & 20 & 1 & 51 & 94 & 2,27 \\
\hline Ps.lutzii & 1 & 0 & 1 & 0 & 0 & 0 & 0 & 0 & 0 & 0 & 11 & 13 & 0,31 \\
\hline Ru.humboldt & 1 & 0 & 0 & 0 & 0 & 0 & 0 & 0 & 0 & 0 & 0 & 1 & 0,02 \\
\hline Ru.reversa & 0 & 0 & 0 & 0 & 0 & 0 & 1 & 0 & 1 & 0 & 5 & 7 & 0,17 \\
\hline Tr.digitatum & 1 & 0 & 0 & 0 & 0 & 0 & 1 & 3 & 1 & 3 & 1 & 10 & 0,24 \\
\hline Ur.lowii & 0 & 0 & 1 & 0 & 0 & 0 & 0 & 0 & 4 & 0 & 0 & 5 & 0,12 \\
\hline Ur.mathesoni & 0 & 0 & 1 & 0 & 0 & 0 & 0 & 0 & 0 & 0 & 0 & 1 & 0,02 \\
\hline Wy pertinans & 1 & 0 & 0 & 0 & 0 & 0 & 0 & 0 & 0 & 0 & 0 & 1 & 0,02 \\
\hline Wy.felicia/pampeites & 0 & 0 & 0 & 0 & 0 & 0 & 0 & 0 & 2 & 0 & 0 & 2 & 0,05 \\
\hline Wy. airosaihowa/uteove & 1 & 0 & 0 & 0 & 0 & 0 & 0 & 0 & 0 & 0 & 0 & 1 & 0,02 \\
\hline Wy.confusa & 20 & 16 & 1 & 0 & 0 & 0 & 15 & 7 & 34 & 19 & 27 & 139 & 3,36 \\
\hline \multirow[t]{2}{*}{ Wy.mystes ou finlayi } & 0 & 0 & 0 & 0 & 1 & 0 & 0 & 0 & 0 & 0 & 0 & 1 & 0,02 \\
\hline & 135 & 538 & 342 & 387 & 272 & 32 & 77 & 360 & 726 & 693 & 581 & 4143 & 100 \\
\hline
\end{tabular}


Tab.12: Número e frequência relativa $(F)$ de indivíduos coletados em armadilha tipo Shannon em área de mata residual ("mata B" - Fazenda Experimental do IAC Pariquera-Açu) durante o ano de 1993.

\begin{tabular}{|c|c|c|c|c|c|c|c|c|c|c|c|c|c|c|}
\hline Espécie & jan & fev & mar & $a b r$ & maio & jun & jul & ago & set & out & nov & dez & total & $F(\%)$ \\
\hline Ae.fulvus & 15 & 32 & 160 & 51 & 8 & 0 & 0 & 0 & 0 & 20 & 29 & 5 & 320 & 3,14 \\
\hline Ae.gr.Serrat(hast/oli) & 0 & 0 & 0 & 0 & 0 & 0 & 0 & 0 & 0 & 0 & 1 & 3 & 4 & 0,04 \\
\hline Ae.gr.Serrat(sen/nub) & 39 & 242 & 168 & 77 & 56 & 42 & 25 & 127 & 38 & 68 & 168 & 135 & 1185 & 11,6 \\
\hline Ae.nubilus & 0 & 0 & 0 & 0 & 0 & 0 & 0 & 1 & 0 & 0 & 0 & 0 & 1 & 0,01 \\
\hline Ae. oligopistus & 0 & 0 & 1 & 0 & 0 & 0 & 0 & 0 & 0 & 0 & 0 & 0 & 1 & 0,01 \\
\hline Ae.scapularis & 41 & 167 & 59 & 21 & 14 & 23 & 7 & 19 & 6 & 138 & 4 & 49 & 548 & 5,37 \\
\hline Ae.serratus & 92 & 259 & 167 & 123 & 97 & 184 & 162 & 417 & 109 & 94 & 510 & 302 & 2516 & 24,6 \\
\hline An.cruzii & 2 & 7 & 2 & 4 & 1 & 0 & 2 & 0 & 0 & 7 & 6 & 0 & 34 & 0,33 \\
\hline An.evansae & 0 & 2 & 0 & 2 & 0 & 0 & 0 & 0 & 0 & 0 & 0 & 0 & 4 & 0,04 \\
\hline An.intermedius & 0 & 5 & 0 & 0 & 0 & 0 & 0 & 0 & 0 & 0 & 1 & 0 & 6 & 0,06 \\
\hline An.oswaldoi & 0 & 0 & 0 & 0 & 1 & 0 & 0 & 0 & 0 & 2 & 0 & 0 & 3 & 0,03 \\
\hline An.prox.mediopunctatus & 5 & 15 & 11 & 13 & 5 & 0 & 1 & 3 & 1 & 3 & 2 & 0 & 59 & 0,58 \\
\hline Cq.chrysonotum & 0 & 6 & 0 & 0 & 0 & 0 & 0 & 0 & 1 & 7 & 7 & 0 & 21 & 0,21 \\
\hline Cq.chrysonotum ou albifera & 35 & 202 & 298 & 205 & 1 & 0 & 0 & 0 & 2 & 19 & 132 & 28 & 922 & 9,03 \\
\hline Cq.hermanoi & 0 & 0 & 0 & 0 & 0 & 0 & 0 & 0 & 0 & 0 & 3 & 0 & 3 & 0,03 \\
\hline Cq.juxtemansonia & 0 & 0 & 1 & 0 & 0 & 0 & 0 & 0 & 0 & 0 & 7 & 0 & 8 & 0,08 \\
\hline Cq.nigricans & 0 & 0 & 9 & 0 & 0 & 0 & 0 & 0 & 0 & 0 & 0 & 0 & 9 & 0,09 \\
\hline Cq.venezuelensis & 14 & 38 & 101 & 49 & 4 & 0 & 0 & 0 & 0 & 9 & 29 & 6 & 250 & 2,45 \\
\hline Cx.(Aed.)amezonensis & 0 & 2 & 11 & 0 & 0 & 0 & 0 & 0 & 0 & 0 & 0 & 0 & 13 & 0,13 \\
\hline Cx.(Cux.) gr Coronetor & 3 & 22 & 0 & 1 & 0 & 0 & 1 & 1 & 1 & 8 & 0 & 0 & 37 & 0,36 \\
\hline CX.(Cux.)bidens & 0 & 0 & 0 & 5 & 0 & 0 & 0 & 0 & 0 & 0 & 0 & 0 & 5 & 0,05 \\
\hline CX.(Cux.)chidesteri & 0 & 3 & 0 & 1 & 0 & 0 & 0 & 0 & 0 & 0 & 0 & 0 & 4 & 0,04 \\
\hline CX. (Cux.)declarator & 0 & 0 & 0 & 0 & 0 & 0 & 0 & 8 & 0 & 0 & 0 & 0 & 8 & 0,08 \\
\hline Cx., Cux.)dolosus & 0 & 0 & 2 & 0 & 0 & 0 & 0 & 0 & 0 & 0 & 0 & 0 & 2 & 0,02 \\
\hline Cx.(Cux.)/ygrus & 0 & 0 & 0 & 0 & 0 & 0 & 0 & 11 & 0 & 0 & 0 & 0 & 11 & 0,11 \\
\hline Cx.(Cux.)mollis & 0 & 4 & 3 & 1 & 0 & 0 & 0 & 1 & 0 & 0 & 0 & 0 & 9 & 0,09 \\
\hline$C_{x}$ (Cux.)nigripalpus & 1 & 80 & 177 & 5 & 0 & 2 & 0 & 8 & 1 & 14 & 1 & 0 & 289 & 2,83 \\
\hline Cx.(Cux.)sp. & 7 & 28 & 14 & 1 & 2 & 0 & 0 & 6 & 0 & 6 & 0 & 4 & 68 & 0,67 \\
\hline Cx.(Lut.)bigoti & 1 & 0 & 0 & 0 & 0 & 0 & 0 & 0 & 0 & 0 & 0 & 0 & 1 & 0,01 \\
\hline Cx.(Mcx.)imitator & 0 & 0 & 0 & 0 & 0 & 0 & 0 & 2 & 0 & 0 & 0 & 0 & 2 & 0.02 \\
\hline$C \times .(M c x$.$) neglectus$ & 0 & 0 & 0 & 0 & 0 & 0 & 0 & 1 & 0 & 0 & 0 & 0 & 1 & 0,01 \\
\hline Cx.(Mel.)eureonotatus & 1 & 0 & 1 & 1 & 0 & 0 & 0 & 0 & 0 & 3 & 0 & 0 & 6 & 0,06 \\
\hline CX.(Mel.)bastagarius & 0 & 5 & 3 & 4 & 0 & 0 & 0 & 1 & 0 & 0 & 5 & 0 & 18 & 0,18 \\
\hline Cx.(Mel.)caudelli & 0 & 0 & 5 & 0 & 0 & 0 & 0 & 0 & 0 & 0 & 0 & 0 & 5 & 0,05 \\
\hline Cx.(Mel.)delpontei & 0 & 1 & 0 & 0 & 0 & 0 & 0 & 0 & 0 & 0 & 0 & 0 & 1 & 0,01 \\
\hline Cx.(Mel.)distinguendus & 0 & 0 & 1 & 0 & 0 & 1 & 0 & 0 & 0 & 0 & 0 & 0 & 2 & 0,02 \\
\hline Cx.(Mel.)eastor & 0 & 1 & 1 & 1 & 0 & 0 & 0 & 0 & 0 & 0 & 0 & 0 & 3 & 0.03 \\
\hline Cx.(Mel.)evansae & 0 & 1 & 7 & 5 & 0 & 0 & 0 & 0 & 0 & 0 & 0 & 0 & 13 & 0,13 \\
\hline Cx.(Mel.)ffaurani & 0 & 0 & 3 & 0 & 0 & 0 & 0 & 0 & 0 & 0 & 0 & 0 & 3 & 0,03 \\
\hline Cx.(Mel.)ganaoi & 0 & 0 & 1 & 0 & 0 & 0 & 0 & 1 & 0 & 0 & 0 & 0 & 2 & 0,02 \\
\hline CX.(Mel.)gnupo Atratus & 3 & 2 & 0 & 5 & 1 & 0 & 0 & 1 & 0 & 4 & 0 & 0 & 16 & 0,16 \\
\hline Cx.(Mel.)grupo Intrincatus & 0 & 0 & 6 & 0 & 1 & 0 & 0 & 0 & 0 & 0 & 0 & 0 & 7 & 0,07 \\
\hline Cx.(Mel.)grupo Pilosus & 1 & 3 & 10 & 4 & 0 & 0 & 0 & 2 & 2 & 0 & 1 & 0 & 23 & 0.23 \\
\hline Cx.(Mel.)misionensis & 0 & 0 & 3 & 0 & 0 & 0 & 0 & 0 & 1 & 0 & 0 & 0 & 4 & 0,04 \\
\hline CX.(Mel.)oceilatus & 0 & 0 & 0 & 0 & 0 & 0 & 0 & 0 & 0 & 0 & 0 & 4 & 4 & 0,04 \\
\hline Cx.(Mel.)ocossa & 0 & 0 & 1 & 0 & 0 & 1 & 0 & 0 & 0 & 0 & 0 & 0 & 2 & 0,02 \\
\hline Cx.(Mel.)oedipus & 2 & 1 & 1 & 0 & 0 & 0 & 0 & 0 & 0 & 0 & 0 & 0 & 4 & 0,04 \\
\hline Cx.(Mel.)orfilai & 0 & 0 & 6 & 0 & 0 & 0 & 0 & 0 & 0 & 0 & 0 & 0 & 6 & 0,06 \\
\hline
\end{tabular}


(cont.)

\begin{tabular}{|c|c|c|c|c|c|c|c|c|c|c|c|c|c|c|}
\hline Espécie & jan & fev & mar & $a b r$ & maio & jun & jul & ago & set & out & nov & dez & total & $\overline{F(\%)}$ \\
\hline Cx.(Mel.)pedroi & 0 & 1 & 3 & 0 & 0 & 0 & 1 & 0 & 0 & 0 & 0 & 0 & 5 & 0,05 \\
\hline Cx.(Mel.)plectoparpe & 0 & 0 & 1 & 0 & 0 & 0 & 0 & 0 & 0 & 0 & 0 & 0 & 1 & 0,01 \\
\hline Cx.(Mel.)rabelloi & 0 & 0 & 0 & 0 & 1 & 0 & 0 & 0 & 0 & 0 & 0 & 0 & 1 & 0,01 \\
\hline CX.(Mel.)ribeirensis & 55 & 106 & 233 & 60 & 14 & 1 & 3 & 6 & 24 & 13 & 18 & 22 & 555 & 5,44 \\
\hline Cx.(Mel.)sacchettee & 15 & 17 & 415 & 302 & 147 & 0 & 0 & 2 & 119 & 19 & 8 & 13 & 1057 & 10.4 \\
\hline Cx.(Mel.)sp & 0 & 0 & 1 & 2 & 0 & 0 & 0 & 0 & 0 & 0 & 0 & 0 & 3 & 0,03 \\
\hline CX.(Mel.)spissipes & 0 & 1 & 4 & 1 & 1 & 0 & 1 & 0 & 0 & 0 & 0 & 0 & 8 & 0.08 \\
\hline Cx.(Mel.)treniopus & 0 & 11 & 13 & 0 & 2 & 0 & 0 & 2 & 0 & 0 & 1 & 0 & 29 & 0.28 \\
\hline Cx.(Mel.)zeteki & 0 & 0 & 0 & 0 & 0 & 0 & 0 & 0 & 0 & 1 & 0 & 0 & 1 & 0,01 \\
\hline Lidurhami & 17 & 26 & 9 & 5 & 4 & 2 & 1 & 3 & 9 & 0 & 10 & 51 & 137 & 1,34 \\
\hline Li.fiavisetosus & 2 & 4 & 0 & 2 & 0 & 1 & 0 & 1 & 0 & 0 & 0 & 17 & 27 & 0.26 \\
\hline Ma.(Man.)indubitans & 6 & 2 & 16 & 2 & 2 & 0 & 0 & 0 & 2 & 2 & 0 & 0 & 32 & 0,31 \\
\hline Ma.(Man.)sp & 0 & 0 & 7 & 2 & 1 & 0 & 0 & 0 & 2 & 3 & 0 & 0 & 15 & 0,15 \\
\hline Ma.(Man.)titillans & 0 & 1 & 0 & 0 & 0 & 0 & 0 & 0 & 0 & 0 & 0 & 0 & 1 & 0,01 \\
\hline Ph.prox. paimateldiabolica & 0 & 0 & 0 & 0 & 0 & 0 & 0 & 0 & 0 & 0 & 0 & 1 & 1 & 0.01 \\
\hline Ph.quasilongirastris & 5 & 1 & 3 & 2 & 0 & 0 & 1 & 0 & 0 & 5 & 22 & 3 & 42 & 0,41 \\
\hline Ps.albigenu & 48 & 73 & 81 & 9 & 0 & 0 & 0 & 0 & 0 & 48 & 0 & 0 & 259 & 2.54 \\
\hline Ps.albigenutvaripes & 52 & 8 & 0 & 0 & 0 & 0 & 0 & 0 & 0 & 6 & 0 & 0 & 66 & 0,65 \\
\hline Ps.albipes & 0 & 0 & 16 & 1 & 0 & 0 & 0 & 0 & 0 & 251 & 3 & 0 & 271 & 2.66 \\
\hline Ps.ferox & 24 & 74 & 85 & 6 & 2 & 0 & 1 & 0 & 0 & 135 & 29 & 31 & 387 & 3,79 \\
\hline Ps.lutzii & 3 & 3 & 9 & 0 & 0 & 0 & 0 & 0 & 0 & 3 & 0 & 0 & 18 & 0.18 \\
\hline Ru.prox. reversa & 0 & 0 & 9 & 0 & 0 & 0 & 0 & 0 & 0 & 0 & 0 & 0 & 9 & 0,09 \\
\hline Ru.reversa & 2 & 1 & 0 & 2 & 0 & 0 & 1 & 1 & 3 & 7 & 0 & 0 & 17 & 0,17 \\
\hline Tr.digitatum & 0 & 1 & 0 & 0 & 0 & 2 & 0 & 0 & 1 & 0 & 0 & 3 & 7 & 0.07 \\
\hline Ur.calosomata & 0 & 1 & 0 & 2 & 0 & 1 & 0 & 0 & 0 & 2 & 0 & 0 & 6 & 0,06 \\
\hline Ur.geometrice & 0 & 0 & 0 & 0 & 0 & 1 & 0 & 0 & 0 & 0 & 0 & 2 & 3 & 0.03 \\
\hline Ur.mathesoni & 0 & 0 & 3 & 0 & 0 & 0 & 0 & 0 & 0 & 0 & 0 & 0 & 3 & 0.03 \\
\hline Ur.nataliae & 0 & 0 & 2 & 0 & 0 & 0 & 0 & 0 & 0 & 0 & 0 & 0 & 2 & 0.02 \\
\hline Wy. felicia/pampeites & 5 & 1 & 12 & 4 & 0 & 0 & 0 & 0 & 0 & 4 & 0 & 2 & 28 & 0,27 \\
\hline Wy.confusa & 47 & 269 & 169 & 53 & 25 & 8 & 1 & 2 & 62 & 20 & 16 & 79 & 751 & 7.36 \\
\hline \multirow[t]{2}{*}{ Wy mystes ou finlayi } & 1 & 0 & 1 & 0 & 0 & 0 & 0 & 0 & 0 & 0 & 0 & 0 & 2 & 0.02 \\
\hline & 544 & 1729 & 5 & & 390 & 269 & 208 & 630 & 384 & 921 & 1013 & 760 & 10207 & 100 \\
\hline
\end{tabular}


Tab.13: Número e frequência relativa $(F)$ de individuos coletados em armadilha tipo Shannon em área de mata residual ("mata B" - Fazenda Experimental do IAC Pariquera-Açu) durante o ano de 1994.

\begin{tabular}{|c|c|c|c|c|c|c|c|c|c|c|c|c|c|}
\hline Espécie & jan & fev & $\operatorname{mar}$ & $a b r$ & maio & jun & jul & ago & set & out & nov & dez & total $F(\%)$ \\
\hline Ae.albopictus & 0 & 0 & 0 & 1 & 0 & 0 & 0 & 0 & 0 & 0 & 0 & 0 & 10,02 \\
\hline Aeffulvus & 14 & 80 & 27 & 7 & 0 & 0 & 0 & 0 & 0 & 0 & 0 & 0 & 1282,73 \\
\hline Ae.gr.Serrat(hastroli) & 0 & 0 & 1 & 0 & 1 & 0 & 0 & 0 & 0 & 0 & 0 & 0 & 20,04 \\
\hline Ae.gr.Serrat(serr/nub) & 101 & 62 & 129 & 79 & 57 & 9 & 15 & 13 & 11 & 0 & 4 & 10 & 49010,5 \\
\hline Ae.sp & 0 & 0 & 0 & 1 & 0 & 0 & 0 & 0 & 0 & 0 & 0 & 0 & 10,02 \\
\hline Ae.scapularis & 13 & 33 & 45 & 30 & 21 & 4 & 11 & 21 & 4 & 1 & 9 & 6 & 1984,23 \\
\hline Ae.serratus & 104 & 87 & 105 & 47 & 42 & 15 & 14 & 46 & 10 & 0 & 19 & 57 & 54611,7 \\
\hline An bellator & 3 & 0 & 39 & 0 & 0 & 0 & 0 & 0 & 0 & 0 & 0 & 0 & $42 \quad 0,9$ \\
\hline An.cruzii & 18 & 65 & 127 & 0 & 0 & 0 & 0 & 0 & 0 & 1 & 2 & 2 & 2154,59 \\
\hline An.evansae & 0 & 0 & 1 & 0 & 0 & 0 & 0 & 0 & 0 & 0 & 0 & 0 & 10,02 \\
\hline An.fiuminensis & 0 & 0 & 0 & 0 & 1 & 0 & 0 & 0 & 0 & 0 & 0 & 0 & 10,02 \\
\hline An.prox.mediopunctatus & 1 & 2 & 0 & 0 & 5 & 1 & 0 & 2 & 1 & 1 & 1 & 0 & 140,3 \\
\hline Antriannulatus & 0 & 0 & 1 & 1 & 0 & 0 & 0 & 0 & 0 & 0 & 0 & 0 & 20,04 \\
\hline Cq.albicosta & 2 & 0 & 0 & 0 & 0 & 0 & 0 & 0 & 0 & 0 & 0 & 0 & 20,04 \\
\hline Cq.chrysonotum & 0 & 0 & 0 & 0 & 0 & 0 & 0 & 0 & 15 & 0 & 1 & 0 & 160,34 \\
\hline Cq.chrysonotumialbifera & 33 & 54 & 51 & 62 & 1 & 0 & 0 & 0 & 0 & 0 & 35 & 7 & 2435,19 \\
\hline Cq.hermanoi & 2 & 2 & 1 & 0 & 0 & 0 & 0 & 0 & 0 & 0 & 0 & 0 & 50,11 \\
\hline Cq.juxtamansonia & 0 & 1 & 1 & 1 & 0 & 0 & 0 & 0 & 0 & 0 & 0 & 0 & 30,06 \\
\hline Cq.venezuelensis & 31 & 18 & 13 & 2 & 2 & 0 & 0 & 0 & 1 & 0 & 6 & 4 & 771,64 \\
\hline Cx.(Aed.)amazonensis & 0 & 1 & 2 & 0 & 0 & 0 & 0 & 0 & 0 & 0 & 0 & 0 & 30,06 \\
\hline Cx.(Cux.) gr Coronator & 0 & 0 & 10 & 0 & 0 & 0 & 2 & 0 & 0 & 1 & 2 & 0 & 150,32 \\
\hline Cx.(Cux.)bidens & 0 & 0 & 1 & 0 & 0 & 0 & 0 & 0 & 0 & 0 & 0 & 0 & 10,02 \\
\hline Cx. (Cux.)declarator & 0 & 0 & 0 & 0 & 2 & 0 & 0 & 0 & 1 & 0 & 0 & 0 & 30,06 \\
\hline Cx. (Cux.)dolosus/eduardoi & 0 & 0 & 1 & 0 & 0 & 0 & 1 & 1 & 0 & 0 & 0 & 0 & 30,06 \\
\hline Cx.(Cux.)mollis & 0 & 0 & 0 & 0 & 0 & 1 & 0 & 0 & 0 & 0 & 0 & 0 & 10,02 \\
\hline CX.(Cux.) Inigripalpus & 0 & 5 & 11 & 3 & 1 & 0 & 0 & 0 & 0 & 0 & 1 & 0 & 210,45 \\
\hline$C_{x}($ Cux.)sp. & 0 & 2 & 0 & 1 & 3 & 1 & 0 & 0 & 1 & 0 & 3 & 4 & 150,32 \\
\hline$C_{x}(M c x$.$) gr. pleuristriatus$ & 0 & 0 & 0 & 0 & 0 & 0 & 0 & 0 & 0 & 0 & 0 & 1 & 10,02 \\
\hline Cx. $(M c x$.$) imitator$ & 0 & 0 & 0 & 0 & 0 & 0 & 0 & 0 & 1 & 0 & 0 & 0 & 10,02 \\
\hline Cx. (Mcx.)neglectus & 0 & 0 & 0 & 0 & 0 & 0 & 0 & 0 & 0 & 0 & 1 & 0 & 10,02 \\
\hline Cx.(Mel.)bastagerius & 7 & 0 & 1 & 1 & 1 & 0 & 0 & 1 & 0 & 0 & 0 & 0 & 110,23 \\
\hline CX.(Mel.)caudelli & 0 & 0 & 2 & 0 & 0 & 0 & 0 & 0 & 0 & 0 & 0 & 0 & 20,04 \\
\hline Cx.(Mel.)dunni & 0 & 0 & 4 & 0 & 0 & 0 & 0 & 1 & 0 & 0 & 0 & 0 & 50.11 \\
\hline Cx.(Mel.)evansee & 0 & 0 & 3 & 2 & 0 & 0 & 0 & 0 & 0 & 0 & 0 & 0 & 50.11 \\
\hline Cx.(Mel.)fourani & 0 & 0 & 1 & 0 & 0 & 0 & 0 & 0 & 0 & 0 & 0 & 0 & 10,02 \\
\hline Cx.(Mel.)galvaoi & 0 & 0 & 0 & 0 & 0 & 0 & 0 & 1 & 0 & 0 & 0 & 0 & 10,02 \\
\hline Cx.(Mel.)gnupo Atratus & 0 & 8 & 10 & 6 & 2 & 2 & 0 & 0 & 0 & 0 & 1 & 1 & 300,64 \\
\hline Cx.(Mel.)grupo Intrincatus & 0 & 0 & 1 & 1 & 0 & 0 & 1 & 0 & 0 & 0 & 0 & 0 & 30,06 \\
\hline CX.(Mel.)gnupo Pilosus & 0 & 0 & 6 & 0 & 0 & 0 & 0 & 1 & 0 & 0 & 2 & 1 & $10 \quad 0,21$ \\
\hline Cx.(Mel.)innovator & 0 & 0 & 8 & 0 & 0 & 0 & 0 & 0 & 0 & 0 & 0 & 0 & 80,17 \\
\hline CX. (Mel.)oedipus & 0 & 0 & 1 & 0 & 0 & 0 & 0 & 0 & 0 & 0 & 0 & 0 & 10,02 \\
\hline Cx.(Mel.)pedroi & 1 & 1 & 3 & 0 & 1 & 0 & 0 & 2 & 0 & 0 & 0 & 0 & 80,17 \\
\hline CX.(Mel.)pereyrai & 0 & 0 & 0 & 2 & 0 & 0 & 0 & 0 & 0 & 0 & 0 & 0 & 20,04 \\
\hline CX.(Mel.)pilosus & 0 & 8 & 11 & 0 & 0 & 0 & 0 & 1 & 0 & 0 & 0 & 0 & 200,43 \\
\hline Cx.(Mel.)plectoporpe & 0 & 0 & 1 & 0 & 0 & 0 & 0 & 0 & 0 & 0 & 0 & 0 & 10,02 \\
\hline Cx.(Mel.)ribeirensis & 14 & 23 & 46 & 16 & 16 & 2 & 4 & 4 & 3 & 0 & 44 & 16 & 1884,01 \\
\hline CX.(Mel).jsacchettae & 18 & 3 & 38 & 24 & 51 & 9 & 0 & 40 & 25 & 2 & 4 & 16 & $230 \quad 4,91$ \\
\hline$C_{x}($ Mel.)sp & 0 & 0 & 0 & 0 & 3 & 0 & 0 & 0 & 0 & 0 & 0 & 0 & 30.06 \\
\hline
\end{tabular}


(cont.)

\begin{tabular}{|c|c|c|c|c|c|c|c|c|c|c|c|c|c|}
\hline Espécie & jan & fev & mar & $a b r$ & maio & jun & jul & ago & set & out & nov & dez & total $F(\%)$ \\
\hline CX.(Mel.)spissipes & 0 & 1 & 5 & 0 & 4 & 0 & 0 & 1 & 0 & 0 & 0 & 0 & 110,23 \\
\hline Cx.(Mel.)tzeniopus & 0 & 0 & 0 & 3 & 0 & 0 & 0 & 0 & 0 & 0 & 1 & 0 & 40,09 \\
\hline Cx. (Mel.)theobaldi V. R. & 2 & 0 & 0 & 0 & 0 & 0 & 1 & 1 & 0 & 0 & 0 & 0 & 40,09 \\
\hline Cx.(Mel.)zetekj & 0 & 0 & 1 & 1 & 0 & 0 & 0 & 0 & 0 & 0 & 0 & 0 & 20,04 \\
\hline Li.duhemi & 180 & 92 & 23 & 5 & 6 & 2 & 21 & 5 & 16 & 12 & 26 & 74 & 4629,87 \\
\hline Li.fiavisetosus & 47 & 6 & 6 & 0 & 0 & 0 & 5 & 13 & 0 & 1 & 5 & 1 & 841,79 \\
\hline Me.(Man.)fiaveola & 0 & 0 & 0 & 0 & 0 & 0 & 0 & 0 & 0 & 0 & 0 & 1 & 10,02 \\
\hline Ma.(Men.)indubitans & 0 & 1 & 9 & 2 & 1 & 0 & 0 & 0 & 0 & 0 & 1 & 0 & 140,3 \\
\hline Ma.(Man.)sp & 0 & 0 & 1 & 1 & 0 & 0 & 0 & 0 & 1 & 0 & 1 & 0 & 40,09 \\
\hline Ph.prox. palmata/diabolica & 0 & 0 & 0 & 0 & 0 & 0 & 0 & 0 & 0 & 0 & 0 & 2 & 20,04 \\
\hline Ph.prox. theobaldi & 0 & 0 & 0 & 0 & 0 & 0 & 0 & 0 & 0 & 0 & 0 & 7 & 70,15 \\
\hline Ph.denisi & 0 & 0 & 0 & 0 & 0 & 0 & 0 & 0 & 0 & 0 & 0 & 4 & 40,09 \\
\hline Ph.quasilongirostris & 0 & 1 & 0 & 0 & 0 & 0 & 0 & 0 & 3 & 0 & 2 & 4 & 100,21 \\
\hline Ph.theobeldi & 0 & 0 & 0 & 1 & 0 & 0 & 4 & 0 & 1 & 0 & 5 & 3 & $14 \quad 0.3$ \\
\hline Phoniomyia sp & 0 & 0 & 0 & 1 & 0 & 0 & 0 & 0 & 0 & 0 & 0 & 0 & 10,02 \\
\hline Ps.albigenu & 6 & 441 & 167 & 0 & 0 & 0 & 0 & 0 & 0 & 0 & 0 & 0 & 61413,1 \\
\hline Ps.albigenutvaripes & 7 & 27 & 39 & 1 & 0 & 0 & 0 & 0 & 0 & 0 & 0 & 0 & 741,58 \\
\hline Ps.elbipes & 6 & 36 & 0 & 0 & 0 & 0 & 0 & 0 & 0 & 0 & 0 & 0 & 420,9 \\
\hline Ps cingulata & 0 & 0 & 0 & 0 & 1 & 0 & 0 & 0 & 0 & 0 & 0 & 0 & 10,02 \\
\hline Ps.ferox & 44 & 67 & 18 & 5 & 1 & 0 & 0 & 0 & 0 & 0 & 3 & 12 & $150 \quad 3,2$ \\
\hline Ps.lutzii & 0 & 1 & 0 & 0 & 0 & 0 & 0 & 0 & 0 & 0 & 0 & 3 & 40,09 \\
\hline Ru.reverse & 0 & 6 & 0 & 0 & 0 & 0 & 0 & 3 & 1 & 0 & 5 & 3 & 180,38 \\
\hline Ru.theobadi & 0 & 0 & 0 & 0 & 0 & 0 & 0 & 0 & 0 & 0 & 1 & 0 & 10,02 \\
\hline Sa.chloropterus & 0 & 0 & 1 & 0 & 0 & 0 & 0 & 0 & 0 & 0 & 0 & 0 & 10,02 \\
\hline Tr.digitatum & 3 & 0 & 0 & 0 & 1 & 0 & 0 & 1 & 0 & 0 & 4 & 4 & 130,28 \\
\hline Ur.calosomata & 0 & 0 & 0 & 1 & 0 & 0 & 0 & 1 & 0 & 0 & 0 & 0 & 20,04 \\
\hline Ur.nateliae & 0 & 0 & 0 & 0 & 0 & 0 & 0 & 0 & 1 & 0 & 0 & 0 & 10,02 \\
\hline Wy.feliciadpampeites & 0 & 4 & 0 & 1 & 0 & 1 & 0 & 0 & 0 & 1 & 1 & 12 & 200,43 \\
\hline Wy.aporonoma & 0 & 3 & 0 & 0 & 0 & 0 & 1 & 2 & 0 & 0 & 1 & 0 & 70,15 \\
\hline Wy.confusa & 36 & 124 & 33 & 40 & 21 & 1 & 28 & 28 & 14 & 22 & 43 & 134 & 52411,2 \\
\hline Wy.mystes ou finlayi & 0 & 2 & 0 & 0 & 0 & 0 & 0 & 0 & 0 & 1 & 2 & 0 & 50.11 \\
\hline Wy.shamoni & 0 & 0 & 0 & 0 & 0 & 0 & 0 & 0 & 0 & 1 & 0 & 0 & 10.02 \\
\hline
\end{tabular}


Tab.14: Número e frequência relativa $(F)$ de indivíduos coletados em armadilha tipo Shannon em área de mata residual ("mata B" Fazenda Experimental do IAC -Pariquera-Açu) no periodo de fevereiro de 1992 a dezembro de 1994.

\begin{tabular}{|c|c|c|c|c|c|}
\hline Especie & 92 & 93 & 94 & Ntotal & $F(\%)$ \\
\hline Ae.albopictus & 0 & 0 & 1 & 1 & 0,01 \\
\hline Ae.crinifer & 2 & 0 & 0 & 2 & 0,01 \\
\hline Ae.fulvus & 38 & 320 & 128 & 486 & 2,55 \\
\hline Ae.gr. Serrat(hastioli) & 2 & 4 & 2 & 8 & 0,04 \\
\hline Ae.gr.Serrat(sen/mub) & 397 & 1185 & 490 & 2072 & 10,9 \\
\hline Ae.nubilus & 0 & 1 & 0 & 1 & 0,01 \\
\hline Ae.oligopistus & 2 & 1 & 0 & 3 & 0,02 \\
\hline Ae.prox.perventor ou hortator & 0 & 0 & 1 & 1 & 0,01 \\
\hline Ae.scapularis & 322 & 548 & 198 & 1068 & 5,61 \\
\hline Ae.serretus & 927 & 2516 & 546 & 3989 & 21 \\
\hline An.bellator & 0 & 0 & 42 & 42 & 0,22 \\
\hline Ancruzii & 25 & 34 & 215 & 274 & 1,44 \\
\hline An evansae & 0 & 4 & 1 & 5 & 0,03 \\
\hline An.fluminensis & 0 & 0 & 1 & 1 & 0,01 \\
\hline An.grupo punctimacula & 1 & 0 & 0 & 1 & 0,01 \\
\hline An.intermedius & 2 & 6 & 0 & 8 & 0,04 \\
\hline An.oswaldoi & 1 & 3 & 0 & 4 & 0,02 \\
\hline An.prox.mediopunctatus & 49 & 59 & 14 & 122 & 0,64 \\
\hline An.triannulatus & 0 & 0 & 2 & 2 & 0,01 \\
\hline Cq.albicosta & 0 & 0 & 2 & 2 & 0,01 \\
\hline Cq.chrysonotum & 2 & 21 & 16 & 39 & 0,2 \\
\hline Cq.chrysonotum ou albifera & 688 & 922 & 243 & 1853 & 9,74 \\
\hline Cq.hermanoi & 2 & 3 & 5 & 10 & 0,05 \\
\hline Cq.juxtamansonia & 0 & 8 & 3 & 11 & 0,06 \\
\hline Cq.nigricans & 0 & 9 & 0 & 9 & 0,05 \\
\hline Cq.venezuelensis & 149 & 250 & 77 & 476 & 2,5 \\
\hline Cx.(Aed.)amazonensis & 6 & 13 & 3 & 22 & 0,12 \\
\hline Cx. (Cux.) gr Coron (coron ou usqu) & 42 & 37 & 15 & 94 & 0,49 \\
\hline$C x .($ Cux.)bidens & 0 & 5 & 1 & 6 & 0,03 \\
\hline Cx.(Cux.)chidesteri & 1 & 4 & 0 & 5 & 0,03 \\
\hline Cx.(Cux.)declarator & 2 & 8 & 0 & 10 & 0,05 \\
\hline$C x .(C u x$,$) dolosus$ & 0 & 2 & 3 & 5 & 0,03 \\
\hline Cx.(Cux.)dolosus/eduardoi & 4 & 0 & 3 & 7 & 0,04 \\
\hline Cx.(Cux.)/ygrus & 0 & 11 & 0 & 11 & 0,06 \\
\hline Cx.(Cux.)mollis & 5 & 9 & 1 & 15 & 0,08 \\
\hline Cx.(Cux.)nigripalpus & 66 & 289 & 21 & 376 & 1,98 \\
\hline Cx.(Cux.)sp. & 9 & 68 & 15 & 92 & 0,48 \\
\hline CX.(Lut)bigoti & 1 & 1 & 0 & 2 & 0.01 \\
\hline Cx.(Mcx.) gr. Pleuristriatus & 0 & 0 & 1 & 1 & 0,01 \\
\hline Cx.(NCx.)grupo imitator & 39 & 0 & 0 & 39 & 0,2 \\
\hline CX. (MCx.)imitator & 0 & 2 & 1 & 3 & 0,02 \\
\hline CX. $(M c x$.)neglectus & 0 & 1 & 1 & 2 & 0,01 \\
\hline Cx.(Mel.)aliciae & 1 & 0 & 0 & 1 & 0,01 \\
\hline CX. (Mel.)eureonotatus & 1 & 6 & 0 & 7 & 0,04 \\
\hline Cx.(Mel.)bastagarius & 9 & 18 & 11 & 38 & 0,2 \\
\hline CX. (Mel.)caudelli & 0 & 5 & 2 & 7 & 0,04 \\
\hline Cx.(Mel.)delpontei & 0 & 1 & 0 & 1 & 0,01 \\
\hline
\end{tabular}


(cont.)

\begin{tabular}{|c|c|c|c|c|c|}
\hline Espécie & 92 & 93 & 94 & Ntotal & $F(\%)$ \\
\hline Cx.(Mel.)distinguendus & 0 & 2 & 0 & 2 & 0,01 \\
\hline Cx.(Mel.)dunni & 3 & 0 & 5 & 8 & 0,04 \\
\hline CX.(Mel.)eastor & 0 & 3 & 0 & 3 & 0,02 \\
\hline Cx.(Mel.)evensae & 1 & 13 & 5 & 19 & 0,1 \\
\hline CX.(Mel.)faureni & 0 & 3 & 1 & 4 & 0,02 \\
\hline Cx.(Mel.)gakaoi & 2 & 2 & 1 & 5 & 0,03 \\
\hline Cx.(Mel.)gnupo Atratus & 54 & 16 & 30 & 100 & 0,53 \\
\hline CX.(Mel.)gnupo Intrincatus & 0 & 7 & 3 & 10 & 0,05 \\
\hline CX.(Mel.)gnupo Pilosus & 12 & 23 & 10 & 45 & 0,24 \\
\hline Cx.(Mel.)innovator & 6 & 0 & 8 & 14 & 0,07 \\
\hline Cx.(Mel.)misionensis & 148 & 4 & 0 & 152 & 0,8 \\
\hline Cx.(Mel.)ocellatus & 0 & 4 & 0 & 4 & 0,02 \\
\hline Cx.(Mel.)ocossa & 0 & 2 & 0 & 2 & 0,01 \\
\hline Cx.(Mel.)oedipus & 15 & 4 & 1 & 20 & 0,11 \\
\hline Cx.(Mel.)orfilai & 0 & 6 & 0 & 6 & 0,03 \\
\hline Cx.(Mel.)pedroi & 9 & 5 & 8 & 22 & 0,12 \\
\hline CX.(Mel.)pereyrai & 0 & 0 & 2 & 2 & 0,01 \\
\hline Cx.(Mel.)pilosus & 8 & 0 & 20 & 28 & 0,15 \\
\hline Cx.(Mel.)plectoporpe & 4 & 1 & 1 & 6 & 0,03 \\
\hline Cx.(Mel.)rebelloi & 0 & 1 & 0 & 1 & 0,01 \\
\hline Cx.(Mel.)ribeirensis & 290 & 555 & 188 & 1033 & 5,43 \\
\hline CX. (Mel.)sacchettre & 165 & 1057 & 230 & 1452 & 7,63 \\
\hline CX.(Mel.)sp & 1 & 3 & 3 & 7 & 0,04 \\
\hline Cx.(Mel.)spissipes & 17 & 8 & 11 & 36 & 0,19 \\
\hline CX.(Mel.)taeniopus & 5 & 29 & 4 & 38 & 0.2 \\
\hline Cx. (Mel.)theobeldi V. $R$ & 15 & 0 & 4 & 19 & 0,1 \\
\hline Cx.(Mel.)zeteki & 1 & 1 & 2 & 4 & 0,02 \\
\hline Lidurhemi & 77 & 137 & 462 & 676 & 3,55 \\
\hline Lifflavisetosus & 25 & 27 & 84 & 136 & 0,71 \\
\hline Ma.(Man.).fleveola & 0 & 0 & 1 & 1 & 0,01 \\
\hline Ma.(Man.)indubitans & 70 & 32 & 14 & 116 & 0,61 \\
\hline Ma.(Man.)sp & 6 & 15 & 4 & 25 & 0,13 \\
\hline Ma.(Man.)titillans & 0 & 1 & 0 & 1 & 0,01 \\
\hline Ph.prox.fiabellata & 1 & 0 & 0 & 1 & 0,01 \\
\hline Ph.prox. palmataddiabolica & 0 & 1 & 2 & 3 & 0,02 \\
\hline Ph.prox.theobaldi & 0 & 0 & 7 & 7 & 0,04 \\
\hline Ph.devisi & 0 & 0 & 4 & 4 & 0,02 \\
\hline Ph.pelmata/diabolice & $T$ & 0 & 0 & 7 & 0,04 \\
\hline Ph.quasilongirostris & 6 & 42 & 10 & 58 & 0,3 \\
\hline Ph.theobaldi & 0 & 0 & 14 & 14 & 0,07 \\
\hline Phoniomyia sp & 0 & 0 & 1 & 1 & 0,01 \\
\hline Ps.albigenu & 91 & 259 & 614 & 964 & 5,06 \\
\hline Ps.albigenurvaripes & 30 & 66 & 74 & 170 & 0.89 \\
\hline Ps.albipes & 0 & 271 & 42 & 313 & 1,64 \\
\hline Ps.cingulata & 0 & 0 & 1 & 1 & 0,01 \\
\hline Ps.discrucians & 4 & 0 & 0 & 4 & 0.02 \\
\hline Ps.ferox & 94 & 387 & 150 & 631 & 3,32 \\
\hline Ps.lutzï & 13 & 18 & 4 & 35 & 0.18 \\
\hline Ru.humboldt & 1 & 0 & 0 & 1 & 0,01 \\
\hline Ru.prox.reversa & 0 & 9 & 0 & 9 & 0,05 \\
\hline
\end{tabular}




\begin{tabular}{lrrrrr} 
(cont.) & 92 & 93 & 94 & Ntotal & $F(\%)$ \\
\hline Espocie & 7 & 17 & 18 & 42 & 0,22 \\
\hline Ru.reversa & 0 & 0 & 1 & 1 & 0,01 \\
Ru.theobaldi & 0 & 0 & 1 & 1 & 0,01 \\
Se.chtoropterus & 10 & 7 & 13 & 30 & 0,16 \\
Tr.digitatum & 0 & 6 & 2 & 8 & 0,04 \\
Ur.calosomata & 0 & 3 & 0 & 3 & 0,02 \\
Ur.geometrica & 5 & 0 & 0 & 5 & 0,03 \\
Ur.lowii & 1 & 3 & 0 & 4 & 0,02 \\
Ur.methesoni & 0 & 2 & 1 & 3 & 0,02 \\
Ur.nataliae & 1 & 0 & 0 & 1 & 0,01 \\
Wy pertinans & 2 & 28 & 20 & 50 & 0,26 \\
Wy.feliciadpampeites & 1 & 0 & 0 & 1 & 0,01 \\
Wy.eirosaihowa/uteove & 0 & 0 & 7 & 7 & 0,04 \\
Wy.eporonoma & 139 & 751 & 524 & 1414 & 7,43 \\
Wy.contusa & 1 & 2 & 5 & 8 & 0,04 \\
Wy.mystes ou finlayi & 0 & 0 & 1 & 1 & 0,01 \\
Wy.shannoni & 4143 & 10207 & 4683 & 19033 & 100 \\
\hline & & & & &
\end{tabular}

Tab.15: Número e frequência relativa (F) de indivíduos coletados em armadilha tipo Shannon em área de cultivo irrigado de arroz (Fazenda Experimental do IAC Pariquera-Açu) durante o ano de 1992.

\begin{tabular}{|c|c|c|c|c|c|c|c|c|c|c|c|c|c|c|}
\hline Espécie & jan & fev & mar & abr & maio & jun & jul & ago & set & out & nov & $\operatorname{dez}$ & total & $F(\%)$ \\
\hline Ae.albifasciatus & 0 & 0 & 0 & 0 & 0 & 0 & 0 & 1 & 0 & 0 & 0 & 0 & & $\overline{0,01}$ \\
\hline Ae.crinifer & 0 & 0 & 0 & 7 & 28 & 41 & 0 & 5 & 7 & 33 & 49 & 2 & 172 & 0,93 \\
\hline Ae.fulvus & 0 & 0 & 0 & 2 & 5 & 0 & 0 & 0 & 0 & 0 & 0 & 0 & & 0,04 \\
\hline Ae.gr.Serrat(serm/nub) & 0 & 2 & 2 & 19 & 24 & 84 & 6 & 0 & 4 & 0 & 57 & 71 & 269 & 1,45 \\
\hline Ae.scapuleris & 55 & 64 & 293 & 188 & 514 & 594 & 77 & 127 & 129 & 115 & 197 & 137 & 2490 & 13,4 \\
\hline Ae.serratus & 0 & 8 & 1 & 5 & 33 & 68 & 0 & 13 & 4 & 1 & 37 & 107 & 277 & 1,5 \\
\hline An albitersis esp. A & 0 & 106 & 18 & 34 & 25 & 20 & 9 & 3 & 0 & 1 & 4 & 36 & 256 & 1,38 \\
\hline An albitarsis esp. $B$ & 0 & 56 & 190 & 157 & 193 & 684 & 64 & 16 & 83 & 79 & 113 & 187 & 4822 & 9,84 \\
\hline An.(Nys.) sec Angynit/Albim & 1 & 0 & 0 & 0 & 0 & 0 & 0 & 0 & 0 & 2 & 0 & 0 & & 0,02 \\
\hline An.albitarsis & 310 & 495 & 7 & 10 & 21 & 43 & 1 & 3 & 0 & 5 & 3 & 4 & 902 & 4,87 \\
\hline An.cruzii & 0 & 0 & 0 & 1 & 0 & 0 & 0 & 1 & 0 & 0 & 4 & 4 & & 0.05 \\
\hline An.evansae & 2 & 2 & 0 & 0 & 8 & 8 & 0 & 5 & 0 & 4 & 1 & 0 & & 0,16 \\
\hline An.galvaoi & 0 & 0 & 0 & 0 & 3 & 0 & 0 & 6 & 2 & 3 & 0 & 0 & & 0,08 \\
\hline Angrupo punctimacula & 0 & 0 & 0 & 0 & 4 & 14 & 0 & 19 & 0 & 0 & 1 & 0 & & 0,21 \\
\hline Anintermedius & 1 & 1 & 6 & 17 & 106 & 205 & 0 & 0 & 9 & 18 & 5 & 9 & 377 & 2,04 \\
\hline An.oswaldoi & 0 & 0 & 0 & 0 & 3 & 17 & 0 & 1 & 2 & 6 & 2 & 1 & & 0.17 \\
\hline An.prox.mediopunctatus & 0 & 0 & 0 & 0 & 0 & 10 & 0 & 0 & 0 & 0 & 0 & 0 & & 0,05 \\
\hline An.strodei & 0 & 0 & 0 & 2 & 2 & 49 & 4 & 1 & 6 & 11 & 8 & 8 & & 0.49 \\
\hline Antriannulatus & 2 & 0 & 2 & 0 & 0 & 0 & 0 & 0 & 0 & 0 & 0 & 2 & & 0,03 \\
\hline Cq.albicosta & 1 & 0 & 2 & 0 & 0 & 0 & 0 & 0 & 0 & 0 & 0 & 0 & & 0.02 \\
\hline Cq.albifera & 0 & 0 & 0 & 0 & 0 & 0 & 0 & 0 & 0 & 5 & 0 & 0 & & 0.03 \\
\hline Cq.chrysonotum & 0 & 1 & 0 & 0 & 0 & 1 & 0 & 0 & 0 & 4 & 0 & 0 & & 0.03 \\
\hline Cq. chrysonotumbelbifera & 31 & 54 & 46 & 280 & 139 & 135 & 10 & 4 & 13 & 109 & 279 & 276 & 1376 & 7,43 \\
\hline Cq.hermanoi & 7 & 10 & 9 & 42 & 27 & 18 & 0 & 0 & 0 & 20 & 28 & 6 & 167 & 0,9 \\
\hline Cq.juxtamansonia & 47 & 86 & 16 & 143 & 116 & 26 & 1 & 2 & 32 & 172 & 70 & 132 & 843 & 4,55 \\
\hline
\end{tabular}




\begin{tabular}{|c|c|c|c|c|c|c|c|c|c|c|c|c|c|}
\hline Espécie & jan & fer & mar & $a b r$ & maio & jun & jul & ago & set & out & nov & dez & total $F(\%)$ \\
\hline Cq.nigricans & 1 & 20 & 2 & 26 & 0 & 0 & 0 & 0 & 0 & 9 & 10 & 7 & $\begin{array}{ll}75 & 0,4\end{array}$ \\
\hline Cq.venezuelensis & 62 & 64 & 6 & 156 & 441 & 911 & 6 & 11 & 21 & 141 & 264 & 182 & 226512,2 \\
\hline Cx.(Aed.)amazonensis & 0 & 0 & 1 & 2 & 0 & 0 & 0 & 0 & 0 & 1 & 0 & 0 & 40,02 \\
\hline Cx.(Cux.) gr Coronator & 0 & 3 & 0 & 14 & 15 & 140 & 17 & 70 & 29 & 38 & 44 & 30 & 4002,16 \\
\hline Cx.(Cux.)chidesteri & 2 & 1 & 0 & 2 & 0 & 0 & 0 & 0 & 0 & 0 & 0 & 0 & 50,03 \\
\hline Cx.(Cux.)comiger & 0 & 0 & 0 & 0 & 0 & 0 & 0 & 0 & 0 & 3 & 0 & 0 & 30,02 \\
\hline CX.(Cux.)nigripalpus & 1 & 24 & 382 & 59 & 37 & 14 & 6 & 9 & 11 & 7 & 37 & 20 & 6073,28 \\
\hline Cx.(Cux.)sp. & 1 & 4 & 3 & 1 & 13 & 7 & 8 & 2 & 0 & 5 & 0 & 0 & 440,24 \\
\hline Cx.(Lut.)bigoti & 0 & 0 & 0 & 0 & 0 & 0 & 0 & 0 & 1 & 0 & 0 & 0 & 10,01 \\
\hline Cx.(Mel.)aureonotatus & 21 & 53 & 3 & 1 & 2 & 0 & 0 & 0 & 0 & 0 & 0 & 0 & 800,43 \\
\hline Cx.(Mel.)bastagarius & 3 & 5 & 2 & 3 & 1 & 0 & 0 & 6 & 0 & 0 & 0 & 0 & 200,11 \\
\hline Cx.(Mel.)delpontei & 0 & 0 & 0 & 2 & 0 & 0 & 0 & 0 & 0 & 0 & 0 & 0 & 20,01 \\
\hline Cx.(Mel.)gnupo Atratus & 0 & 2 & 0 & 0 & 0 & 0 & 0 & 0 & 0 & 1 & 0 & 0 & 30,02 \\
\hline Cx.(Mel.)gnupo Pilosus & 0 & 3 & 25 & 1 & 8 & 7 & 0 & 1 & 0 & 0 & 0 & 3 & 480,26 \\
\hline Cx.(Mel.)inadmirabilis & 0 & 0 & 0 & 0 & 1 & 0 & 0 & 0 & 0 & 0 & 0 & 0 & 10,01 \\
\hline Cx.(Mel.)ocossa & 2 & 14 & 17 & 14 & 8 & 16 & 0 & 0 & 7 & 6 & 0 & 6 & 900,49 \\
\hline Cx.(Mel.)plectoporpe & 1 & 1 & 0 & 0 & 1 & 0 & 0 & 1 & 0 & 1 & 0 & 0 & 50,03 \\
\hline Cx.(Mel.)nibeirensis & 2 & 23 & 29 & 90 & 128 & 123 & 19 & 81 & 95 & 46 & 0 & 21 & 6573,55 \\
\hline CX.(Mel.)sacchettae & 0 & 4 & 0 & 15 & 5 & 7 & 0 & 1 & 11 & 2 & 0 & 19 & 640,35 \\
\hline Cx.(Mel.)theobaldi V. $R$. & 1 & 7 & 0 & 1 & 0 & 0 & 2 & 1 & 2 & 0 & 0 & 0 & 140,08 \\
\hline Ma.(Man.)fieveola & 0 & 0 & 0 & 0 & 0 & 0 & 0 & 0 & 0 & 0 & 2 & 2 & 40,02 \\
\hline Ma.(Man.)humeralis & 12 & 131 & 45 & 190 & 82 & 105 & 0 & 0 & 3 & 9 & 141 & 20 & 7383,98 \\
\hline Ma.(Man.)indubitans & 21 & 14 & 19 & 155 & 249 & 280 & 28 & 26 & 34 & 38 & 169 & 184 & 12176,57 \\
\hline Ma.(Man.)pseudotitillans & 0 & 4 & 4 & 3 & 2 & 0 & 0 & 0 & 0 & 1 & 0 & 2 & 160,09 \\
\hline Ma.(Man.)sp & 10 & 60 & 32 & 164 & 249 & 578 & 225 & 55 & 54 & 94 & 162 & 187 & 187010,1 \\
\hline Ma.(Man).titillans & 44 & 28 & 0 & 0 & 1 & 0 & 0 & 1 & 0 & 0 & 0 & 21 & 950,51 \\
\hline Ps.albigenu & 6 & 1 & 27 & 55 & 30 & 0 & 0 & 1 & 0 & 0 & 0 & 0 & $120 \quad 0,65$ \\
\hline Ps.albigenu/varipes & 3 & 4 & 3 & 0 & 1 & 0 & 0 & 0 & 0 & 0 & 0 & 0 & 110,06 \\
\hline Ps.ciliata & 1 & 3 & 1 & 2 & 11 & 2 & 13 & 0 & 1 & 6 & 7 & 21 & 680,37 \\
\hline Ps.cingulata & 0 & 0 & 0 & 1 & 2 & 0 & 2 & 2 & 0 & 0 & 1 & 0 & 80,04 \\
\hline Ps.confinnis & 2 & 15 & 44 & 17 & 70 & 11 & 0 & 0 & 0 & 0 & 0 & 0 & 1590,86 \\
\hline Ps.discrucians & 52 & 11 & 97 & 43 & 87 & 18 & 0 & 0 & 0 & 84 & 51 & 161 & 6043,26 \\
\hline Ps.ferox & 0 & 0 & 0 & 0 & 1 & 0 & 0 & 0 & 0 & 0 & 2 & 6 & 90,05 \\
\hline Ur.apicalis & 0 & 0 & 0 & 0 & 0 & 0 & 0 & 0 & 0 & 1 & 0 & 0 & 10,01 \\
\hline Ur.geometrica & 1 & 0 & 3 & 0 & 0 & 0 & 0 & 1 & 0 & 0 & 0 & 0 & 50,03 \\
\hline Ur.pulchemima & 0 & 0 & 0 & 0 & 0 & 0 & 0 & 3 & 1 & 0 & 0 & 0 & 40,02 \\
\hline
\end{tabular}


Tab.16: Número e frequência relativa $(F)$ de individuos coletados em armadilha tipo Shannon em área de cultivo irrigado de arroz (Fazenda Experimental do IAC Pariquera-Açu) durante o ano de 1993

\begin{tabular}{|c|c|c|c|c|c|c|c|c|c|c|c|c|c|c|}
\hline Espécie & jan & fev & mar & $a b r$ & maio & jun & jul & ago & set & out & nov & $\operatorname{dez}$ & total & $F(\%)$ \\
\hline Ad.squamipennis & 0 & 0 & 0 & 1 & 1 & 0 & 1 & 0 & 0 & 0 & 16 & 0 & 19 & 0,039 \\
\hline Ae.criniter & 0 & 0 & 0 & 18 & 0 & 0 & 14 & 5 & 0 & 14 & 0 & 0 & 51 & 0,105 \\
\hline Ae.funus & 1 & 0 & 10 & 1 & 0 & 0 & 0 & 0 & 0 & 0 & 6 & 0 & 18 & 0,037 \\
\hline Ae.gr.Serrat(serr/nub) & 9 & 0 & 0 & 5 & 1 & 0 & 2 & 0 & 0 & 0 & 213 & 0 & 230 & 0,473 \\
\hline Ae.nubilus & 1 & 0 & 0 & 0 & 0 & 0 & 0 & 0 & 0 & 0 & 0 & 0 & 1 & 0,002 \\
\hline Ae.scapularis & 201 & 2 & 519 & 147 & 50 & 34 & 134 & 17 & 21 & 223 & 183 & 21 & 1552 & 3,194 \\
\hline Ae.serratus & 6 & 8 & 0 & 4 & 2 & 1 & 7 & 0 & 0 & 164 & 289 & 0 & 481 & 0,99 \\
\hline An. albitarsis esp. A & 102 & 92 & 759 & 100 & 33 & 7 & 6 & 120 & 0 & 22 & 40 & 67 & 1348 & 2,774 \\
\hline An. albitarsis esp. B & 1385 & 402 & 13249 & 1629 & 143 & 104 & 74 & 0 & 97 & 618 & 1808 & 2015 & 21524 & 44,3 \\
\hline An.(Nys.)secArgyritAlbim & 0 & 0 & 0 & 19 & 2 & 0 & 0 & 0 & 1 & 1 & 0 & 0 & 23 & 0,047 \\
\hline An. albitarsis & 5 & 2 & 967 & 30 & 3 & 6 & 7 & 5 & 1 & 12 & 70 & 144 & 1252 & 2,577 \\
\hline An.cruzï & 0 & 0 & 3 & 0 & 1 & 0 & 0 & 1 & 0 & 10 & 0 & 0 & 15 & 0,031 \\
\hline An evansee & 0 & 1 & 12 & 17 & 0 & 0 & 1 & 1 & 1 & 8 & 0 & 0 & 41 & 0,084 \\
\hline An evansae/galvaoi & 0 & 0 & 1 & 0 & 0 & 0 & 0 & 0 & 0 & 0 & 0 & 0 & 1 & 0,002 \\
\hline Angalvaoi & 0 & 0 & 10 & 9 & 0 & 0 & 2 & 0 & 7 & 36 & 10 & 1 & 75 & 0,154 \\
\hline An grupo punctimacula & 0 & 0 & 15 & 11 & 3 & 1 & 1 & 0 & 0 & 0 & 0 & 0 & 31 & 0.064 \\
\hline An,intermedius & 0 & 0 & 314 & 49 & 11 & 2 & 8 & 1 & 3 & 17 & 2 & 0 & 407 & 0,838 \\
\hline An.oswaldoi & 0 & 1 & 0 & 9 & 0 & 1 & 1 & 2 & 1 & 2 & 4 & 3 & 24 & 0,049 \\
\hline An.prox.mediopunctatus & 0 & 0 & 7 & 49 & 0 & 0 & 0 & 0 & 4 & 1 & 0 & 0 & 61 & 0,126 \\
\hline An.strodei & 0 & 0 & 63 & 16 & 2 & 0 & 0 & 0 & 0 & 12 & 21 & 9 & 123 & 0,253 \\
\hline An.triannulatus & 0 & 0 & 9 & 3 & 0 & 0 & 3 & 0 & 0 & 0 & 3 & 4 & 22 & 0,045 \\
\hline Cq.albicosta & 0 & 1 & 0 & 0 & 0 & 0 & 0 & 0 & 0 & 0 & 0 & 0 & 1 & 0,002 \\
\hline Cq.chrysonotum & 0 & 0 & 7 & 3 & 0 & 0 & 0 & 0 & 0 & 10 & 7 & 24 & 51 & 0,105 \\
\hline Cq.chrysonotumvalbifera & 104 & 82 & 1131 & 1308 & 50 & 18 & 1 & 0 & 47 & 454 & 1738 & 577 & 5510 & 11,34 \\
\hline Cq.hemenoi & 44 & 0 & 24 & 5 & 10 & 0 & 0 & 0 & 0 & 0 & 35 & 68 & 186 & 0,383 \\
\hline Cq.juxtamansonia & 67 & 9 & 410 & 595 & 184 & 13 & 0 & 0 & 10 & 15 & 517 & 79 & 1899 & 3,908 \\
\hline Cq.nignicans & 11 & 0 & 57 & 14 & 0 & 0 & 0 & 0 & 0 & 0 & 181 & 9 & 272 & 0,56 \\
\hline Cq.venezuelensis & 80 & 18 & 525 & 457 & 234 & 99 & 27 & 2 & 13 & 124 & 647 & 289 & 2515 & 5,176 \\
\hline Cx.(Aed.)amazonensis & 0 & 0 & 0 & 1 & 0 & 0 & 0 & 0 & 0 & 0 & 0 & 0 & 1 & 0,002 \\
\hline Cx.(Cux.) gr Coronator & 0 & 0 & 0 & 6 & 7 & 1 & 1 & 5 & 7 & 0 & 6 & 0 & 33 & 0,068 \\
\hline Cx.(Cux.)chidesten & 0 & 0 & 0 & 0 & 5 & 0 & 0 & 0 & 0 & 0 & 0 & 0 & 5 & 0,01 \\
\hline Cx.(Cux.)comiger & 0 & 0 & 0 & 0 & 0 & 0 & 0 & 1 & 0 & 0 & 0 & 0 & 1 & 0,002 \\
\hline Cx.(Cux.)nigripalpus & 0 & 4 & 718 & 10 & 1 & 0 & 9 & 0 & 15 & 0 & 44 & 26 & 827 & 1,702 \\
\hline Cx.(Cux.)sp. & 0 & 0 & 0 & 7 & 0 & 3 & 7 & 0 & 7 & 0 & 9 & 0 & 33 & 0,068 \\
\hline Cx.(Lut.)bigoti & 0 & 0 & 0 & 0 & 0 & 0 & 0 & 0 & 0 & 1 & 0 & 0 & 1 & 0,002 \\
\hline Cx.(Mcx.)grupo imitator & 0 & 0 & 1 & 0 & 0 & 0 & 0 & 0 & 0 & 0 & 0 & 0 & 1 & 0,002 \\
\hline CX.(Mel.)aureonotatus & 13 & 46 & 44 & 3 & 4 & 0 & 0 & 0 & 0 & 0 & 0 & 0 & 110 & 0,226 \\
\hline CX. (Mel.)bastagarius & 0 & 1 & 0 & 2 & 2 & 0 & 0 & 0 & 0 & 0 & 0 & 0 & 5 & 0,01 \\
\hline Cx.(Mel),jelpontei & 0 & 0 & 2 & 1 & 1 & 3 & 0 & 0 & 0 & 0 & 0 & 0 & 7 & 0,014 \\
\hline Cx. (Mel.)grupo Pilosus & 0 & 1 & 17 & 1 & 0 & 0 & 0 & 0 & 0 & 0 & 6 & 0 & 25 & 0,054 \\
\hline Cx.(Mel.)inadmirabilis & 0 & 0 & 0 & 2 & 0 & 0 & 0 & 0 & 0 & 0 & 0 & 0 & 2 & 0.004 \\
\hline Cx.(Mel.)ocossa & 0 & 3 & 65 & 7 & 1 & 3 & 0 & 0 & 0 & 0 & 0 & 10 & 89 & 0,183 \\
\hline Cx.(Mel.)oedipus & 1 & 0 & 0 & 0 & 0 & 0 & 0 & 0 & 0 & 0 & 0 & 0 & 1 & 0.002 \\
\hline Cx.(Mel.)plectoporpe & 0 & 2 & 2 & 2 & 0 & 0 & 0 & 0 & 0 & 0 & 0 & 0 & 6 & 0,012 \\
\hline CX.(Mel.)nbeirensis & 34 & 3 & 156 & 32 & 21 & 29 & 22 & 1 & 1 & 25 & 54 & 29 & 407 & 0.838 \\
\hline Cx. (Mel) sacchettae & 6 & 2 & 243 & 25 & 8 & 9 & 4 & 0 & 0 & 18 & 10 & 18 & 343 & 0,706 \\
\hline Cx. (Mel.)sp & 0 & 1 & 0 & 0 & 0 & 0 & 0 & 0 & 0 & 0 & 0 & 0 & 1 & 0,002 \\
\hline CX.(Mel.)theobaldi V. $R$. & 3 & 0 & 0 & 0 & 0 & 3 & 0 & 0 & 0 & 10 & 0 & 0 & 16 & 0,033 \\
\hline
\end{tabular}


(cont.)

\begin{tabular}{lrrrrrrrrrrrrrrrr}
\hline Espécie & jan & fev & mar & abr maio & jun & jul & ago & set & out & nov & dez & total & $F(\%)$ \\
\hline Cx.(Mel.)zeteki & 0 & 0 & 0 & 1 & 0 & 0 & 0 & 0 & 0 & 0 & 0 & 0 & 1 & 0,002 \\
Li.durtami & 3 & 0 & 0 & 0 & 0 & 0 & 0 & 0 & 0 & 0 & 0 & 0 & 3 & 0,006 \\
Ma.(Man.)cerqueirai & 1 & 0 & 0 & 0 & 0 & 0 & 0 & 0 & 0 & 0 & 0 & 0 & 1 & 0,002 \\
Ma.(Man.)fleveola & 0 & 0 & 0 & 0 & 0 & 0 & 0 & 0 & 0 & 0 & 8 & 4 & 12 & 0,025 \\
Ma.(Man.)humeralis & 35 & 0 & 299 & 63 & 34 & 7 & 3 & 0 & 22 & 20 & 34 & 36 & 553 & 1,138 \\
Ma.(Man.)indubitans & 41 & 11 & 561 & 441 & 220 & 126 & 119 & 13 & 0 & 0 & 717 & 189 & 2438 & 5,017 \\
Ma.(Man.)pseudotitillans & 40 & 3 & 19 & 1 & 1 & 0 & 0 & 0 & 0 & 0 & 9 & 0 & 73 & 0,15 \\
Ma.(Man.)sp & 125 & 31 & 541 & 169 & 280 & 156 & 137 & 17 & 16 & 113 & 591 & 187 & 2363 & 4,863 \\
Ma.(Man.)titillans & 5 & 1 & 16 & 3 & 1 & 0 & 0 & 0 & 0 & 0 & 0 & 0 & 26 & 0,054 \\
Ps.albigenu & 6 & 0 & 496 & 2 & 0 & 0 & 4 & 0 & 0 & 0 & 49 & 18 & 575 & 1,183 \\
Ps.albigenurvaripes & 103 & 0 & 380 & 88 & 0 & 0 & 0 & 0 & 0 & 0 & 0 & 0 & 571 & 1,175 \\
Ps.ciliata & 12 & 0 & 23 & 0 & 1 & 3 & 1 & 0 & 2 & 8 & 12 & 4 & 66 & 0,136 \\
Ps.cingulata & 3 & 0 & 0 & 3 & 1 & 1 & 1 & 0 & 0 & 0 & 0 & 0 & 9 & 0,019 \\
Ps.confinnis & 1 & 0 & 11 & 6 & 11 & 2 & 0 & 0 & 0 & 0 & 4 & 0 & 35 & 0,072 \\
Ps.discrucians & 834 & 134 & 503 & 376 & 0 & 2 & 21 & 0 & 0 & 226 & 30 & 0 & 2126 & 4,375 \\
Ps.ferox & 0 & 0 & 45 & 0 & 0 & 0 & 0 & 0 & 0 & 20 & 0 & 0 & 65 & 0,134 \\
Ur.geometrica & 0 & 1 & 4 & 2 & 0 & 0 & 0 & 0 & 0 & 0 & 0 & 8 & 15 & 0,031 \\
Ur.lowii & 0 & 0 & 3 & 0 & 0 & 0 & 0 & 0 & 0 & 0 & 4 & 3 & 10 & 0,021 \\
Ur.pulchemma & 0 & 0 & 2 & 0 & 0 & 0 & 0 & 0 & 0 & 0 & 0 & 0 & 2 & 0,004 \\
Wy pertinans & 0 & 0 & 0 & 0 & 0 & 0 & 0 & 0 & 0 & 1 & 0 & 0 & 1 & 0,002 \\
\hline & 3282 & 862 & 22243 & 5753 & 1329 & 634 & 618 & 191 & 276 & 2185 & 7377 & 3842 & 48592 & 100 \\
\hline & & & & & & & & & & & &
\end{tabular}

Tab.17: Número e frequência relativa (F) de individuos coletados em armadilha tipo Shannon em área de cultivo irrigado de arroz (Fazenda Experimental do IAC-Pariquera-Açu) no período de janeiro de 1992 a dezembro de 1994.

\begin{tabular}{lrrrr}
\hline Espécie & 92 & 93 & Ntotal & $F(\%)$ \\
\hline Ad.squamipennis & 0 & 19 & 19 & 0,028 \\
Ae.albifasciatus & 1 & 0 & 1 & 0,001 \\
Ae.crinifer & 172 & 51 & 223 & 0,332 \\
Ae.futus & 7 & 18 & 25 & 0,037 \\
Ae.gr.Serrat(serr/nub) & 269 & 230 & 499 & 0,743 \\
Ae.nubilus & 0 & 1 & 1 & 0,001 \\
Ae.scapularis & 2490 & 1552 & 4042 & 6,022 \\
Ae.serratus & 277 & 481 & 758 & 1,129 \\
An.albitarsis esp. A & 256 & 1348 & 1604 & 2,39 \\
An.albitarsis esp. B & 1822 & 21524 & 23346 & 34,78 \\
An.(Nys.) secro Argyrit ou Albim & 3 & 23 & 26 & 0,039 \\
An.albitarsis & 902 & 1252 & 2154 & 3,209 \\
An.cruzii & 10 & 15 & 25 & 0,037 \\
An.evansae & 30 & 41 & 71 & 0,106 \\
An.evansee/gatvaoi & 0 & 1 & 1 & 0,001 \\
An.gaivaoi & 14 & 75 & 89 & 0,133 \\
An.grupo punctimacula & 38 & 31 & 69 & 0,103 \\
An.intermedius & 377 & 407 & 784 & 1,168 \\
An.oswaldoi & 32 & 24 & 56 & 0,083 \\
An.prox.mediopunctatus & 10 & 61 & 71 & 0,106 \\
An.strodei & 91 & 123 & 214 & 0,319 \\
An.triannulatus & 6 & 22 & 28 & 0,042 \\
Cq.elbicoste & 3 & 1 & 4 & 0,006 \\
\hline
\end{tabular}


(cont.)

\begin{tabular}{|c|c|c|c|c|}
\hline Espécie & 92 & 93 & Ntotal & $F(\%)$ \\
\hline Cq. albifera & 5 & 0 & 5 & 0,007 \\
\hline Cq.chrysonotum & 6 & 51 & 57 & 0,085 \\
\hline Cq.chrysonotum ou albifera & 1376 & 5510 & 6886 & 10,26 \\
\hline Cq.hermanoi & 167 & 186 & 353 & 0,526 \\
\hline Cq.juxtamansonia & 843 & 1899 & 2742 & 4,085 \\
\hline Cq.nigricans & 75 & 272 & 347 & 0,517 \\
\hline Cq.venezuelensis & 2265 & 2515 & 4780 & 7,122 \\
\hline Cx.(Aed.)amazonensis & 4 & 1 & 5 & 0,007 \\
\hline CX. (Cux.) gr Coron (coron ou usqu) & 400 & 33 & 433 & 0,645 \\
\hline Cx. (Cux.)chidesteri & 5 & 5 & 10 & 0,015 \\
\hline Cx.(Cux.)comiger & 3 & 1 & 4 & 0,006 \\
\hline Cx.(Cux,)nigripalpus & 607 & 827 & 1434 & 2,137 \\
\hline CX. (Cux.)sp. & 44 & 33 & 77 & 0,115 \\
\hline Cx.(Lut)bigoti & 1 & 1 & 2 & 0,003 \\
\hline CX.(Mcx.)grupo imitator & 0 & 1 & 1 & 0,001 \\
\hline Cx.(Mel.)eureonotatus & 80 & 110 & 190 & 0,283 \\
\hline Cx.(Mel.)bastagarius & 20 & 5 & 25 & 0,037 \\
\hline Cx.(Mel.)delpontei & 2 & 7 & 9 & 0,013 \\
\hline Cx.(Mel.)grupo Atratus & 3 & 0 & 3 & 0,004 \\
\hline CX.(Mel.)grupo Pilosus & 48 & 25 & 73 & 0,109 \\
\hline Cx.(Mel.)inadmirabilis & 1 & 2 & 3 & 0,004 \\
\hline Cx.(Mel.)ocossa & 90 & 89 & 179 & 0,267 \\
\hline CX.(Mel.)oedipus & 0 & 1 & 1 & 0,001 \\
\hline Cx.(Mel.)plectoporpe & 5 & 6 & 11 & 0,016 \\
\hline CX.(Mel.)ribeirensis & 657 & 407 & 1054 & 1,585 \\
\hline CX.(Mel).sacchettae & 64 & 343 & 407 & 0,606 \\
\hline$C x($ Mel.)sp & 0 & 1 & 1 & 0,001 \\
\hline Cx. Mel.)theobaldi V. R. & 14 & 16 & 30 & 0,045 \\
\hline Cx.(Mel.)zeteki & 0 & 1 & 1 & 0,001 \\
\hline Lidurhami & 0 & 3 & 3 & 0,004 \\
\hline Ma.(Men.)cerqueirai & 0 & 1 & 1 & 0,001 \\
\hline Ma.(Man.)fiaveola & 4 & 12 & 16 & 0,024 \\
\hline Ma.(Man.)humeralis & 738 & 553 & 1291 & 1,924 \\
\hline Ma.(Man.)indubitans & 1217 & 2438 & 3655 & 5,446 \\
\hline Ma.(Men.)pseudotitillans & 16 & 73 & 89 & 0,133 \\
\hline Ma.(Man.)sp & 1870 & 2363 & 4233 & 6,307 \\
\hline Ma.(Man.)titillans & 95 & 26 & 121 & 0,18 \\
\hline Ps.albigenu & 120 & 575 & 695 & 1,036 \\
\hline Ps. albigenutvaripes & 11 & 571 & 582 & 0,867 \\
\hline Ps. ciliata & 68 & 66 & 134 & 0,2 \\
\hline Ps. cingulata & 8 & 9 & 17 & 0,025 \\
\hline Ps.confinnis & 159 & 35 & 194 & 0,289 \\
\hline Ps.discrucians & 604 & 2126 & 2730 & 4,068 \\
\hline Ps.ferox & 9 & 65 & 74 & 0,11 \\
\hline Ur.apicalis & 1 & 0 & 1 & 0,001 \\
\hline Ur.geometrica & 5 & 15 & 20 & 0,03 \\
\hline Ur.towii & 0 & 10 & 10 & 0,015 \\
\hline Ur.pulchernime & 4 & 2 & 6 & 0,009 \\
\hline \multirow[t]{2}{*}{ Wy pertinans } & 0 & 1 & 1 & 0,001 \\
\hline & 18524 & 48592 & 67116 & 100 \\
\hline
\end{tabular}


Tab.19: Número e de individuos $(\mathrm{N})$ e de espécies (S) por coleta, número adicionado de espécies (Sadic.) a cada coleta e número acumulado de indivíduos (Nacum.) e espécies (Sacum.) obtidos no período de 12 meses. Coletas em armadilha tipo Shannon em ambiente de mata primária ("Fonte" - Cananéia SP)

\begin{tabular}{cccccc}
\hline Coleta & N & Nacum & S & Sadic & Sacum. \\
\hline Fev/92 & 9646 & 9646 & 46 & 46 & 46 \\
Mar/92 & 2428 & 12074 & 34 & 12 & 58 \\
Abr/92 & 2768 & 14842 & 38 & 12 & 70 \\
Maio/92 & 2918 & 17760 & 20 & 3 & 73 \\
Jun/92 & 4016 & 21776 & 29 & 3 & 76 \\
Jul/92 & 587 & 22363 & 21 & 2 & 78 \\
Ago/92 & 1144 & 23507 & 19 & 1 & 79 \\
Set/92 & 1351 & 24858 & 22 & 2 & 81 \\
Out/92 & 3882 & 28740 & 38 & 4 & 85 \\
Nov/92 & 2351 & 31091 & 34 & 4 & 89 \\
Dez/92 & 5740 & 36831 & 43 & 4 & 93 \\
Jan/93 & 552 & 37383 & 20 & 1 & 94 \\
\hline
\end{tabular}

Tab.20: Número de indivíduos (N) e de espécies (S) por coleta, número adicionado de espécies (Sadic.) a cada coleta e número acumulado de indivíduos (Nacum.) e espécies (Sacum.) obtidos nos respectivos periodos de coleta a cada ano. Coletas em armadilha tipo Shannon em ambiente de mata primária (Sítio Gentil - Cananéia. SP).

\begin{tabular}{|c|c|c|c|c|c|c|}
\hline Ano & Coleta & $N$ & Nacum. & $S$ & Sadic. & Sacum \\
\hline \multirow[t]{7}{*}{93} & Jun & 246 & 246 & 15 & 15 & 15 \\
\hline & Jul & 655 & 901 & 8 & 1 & 16 \\
\hline & Ago & 267 & 1168 & 8 & 4 & 20 \\
\hline & Set & 484 & 1652 & 15 & 4 & 24 \\
\hline & Out & 2036 & 3688 & 18 & 8 & 32 \\
\hline & Nov & 2647 & 6335 & 26 & 9 & 41 \\
\hline & Dez & 1195 & 7530 & 20 & 2 & 43 \\
\hline \multirow[t]{12}{*}{94} & Jan & 758 & 758 & 29 & 29 & 29 \\
\hline & Fev & 1310 & 2068 & 22 & 4 & 33 \\
\hline & Mar & 1112 & 3180 & 21 & 4 & 37 \\
\hline & $\mathrm{Abr}$ & 447 & 3627 & 24 & 4 & 41 \\
\hline & Maio & 1528 & 5155 & 28 & 8 & 49 \\
\hline & Jun & 78 & 5233 & 13 & 1 & 50 \\
\hline & Jul & 45 & 5278 & 10 & 0 & 50 \\
\hline & Ago & 158 & 5436 & 12 & 3 & 53 \\
\hline & Set & 138 & 5574 & 14 & 0 & 53 \\
\hline & Out & 1599 & 7173 & 27 & 9 & 62 \\
\hline & Nov & 1892 & 9065 & 23 & 4 & 66 \\
\hline & Dez & 1795 & 10860 & 23 & 5 & 71 \\
\hline \multirow[t]{4}{*}{95} & Jan & 2274 & 2274 & 21 & 21 & 21 \\
\hline & Fev & 2443 & 4717 & 24 & 12 & 33 \\
\hline & Mar & 2556 & 7273 & 46 & 22 & 55 \\
\hline & $\mathrm{Abr}$ & 927 & 8200 & 30 & 4 & 59 \\
\hline
\end{tabular}


Tab.21: Número de individuos $(\mathrm{N})$ e de espécies (S) por coleta, número adicionado de espécies (Sadic.) a cada coleta e número acumulado de indivíduos (Nacum.) e espécies (Sacum.) obtidos no periodo de 23 meses. Coletas em armadilha tipo Shannon em ambiente de mata primária (Sítio Gentil - Cananéia, SP).

\begin{tabular}{ccccccc}
\hline Ano & Coleta & N & Nacum. & S & Sadic. & Sacum. \\
\hline 93 & Jun & 246 & 246 & 15 & 15 & 15 \\
& Jul & 655 & 901 & 8 & 1 & 16 \\
& Ago & 267 & 1168 & 8 & 4 & 20 \\
& Set & 484 & 1652 & 15 & 4 & 24 \\
& Out & 2036 & 3688 & 18 & 8 & 32 \\
& Nov & 2647 & 6335 & 26 & 9 & 41 \\
& Dez & 1195 & 7530 & 20 & 2 & 43 \\
\hline 94 & Jan & 758 & 8288 & 29 & 5 & 48 \\
& Fev & 1310 & 9598 & 22 & 2 & 50 \\
& Mar & 1112 & 10710 & 21 & 2 & 52 \\
& Abr & 447 & 11157 & 24 & 4 & 56 \\
& Maio & 1528 & 12685 & 28 & 5 & 61 \\
& Jun & 78 & 12763 & 13 & 1 & 62 \\
& Jul & 45 & 12808 & 10 & 0 & 62 \\
& Ago & 158 & 12966 & 12 & 2 & 64 \\
& Set & 138 & 13104 & 14 & 0 & 64 \\
& Out & 1599 & 14703 & 27 & 6 & 70 \\
& Nov & 1892 & 16595 & 23 & 4 & 74 \\
& Dez & 1795 & 18390 & 23 & 5 & 79 \\
\hline 95 & Jan & 2274 & 20664 & 21 & 1 & 80 \\
& Fev & 2443 & 23107 & 24 & 1 & 81 \\
& Mar & 2556 & 25663 & 46 & 4 & 85 \\
& Abr & 927 & 26590 & 30 & 1 & 86 \\
\hline \multirow{6}{*}{9} & & & & &
\end{tabular}


Tab.21: Número de indivíduos $(\mathrm{N})$ e de espécies (S) por coleta, número adicionado de espécies (Sadic.) a cada coleta e número acumulado de indivíduos (Nacum.) e espécies (Sacum.) obtidos no periodo de 23 meses. Coletas em armadilha tipo Shannon em ambiente de mata primária (Sítio Gentil - Cananéia, SP)

\begin{tabular}{|c|c|c|c|c|c|c|}
\hline Ano & Coleta & $N$ & Nacum. & $S$ & Sadic. & Sacum. \\
\hline \multirow[t]{7}{*}{93} & Jun & 246 & 246 & 15 & 15 & 15 \\
\hline & Jul & 655 & 901 & 8 & 1 & 16 \\
\hline & Ago & 267 & 1168 & 8 & 4 & 20 \\
\hline & Set & 484 & 1652 & 15 & 4 & 24 \\
\hline & Out & 2036 & 3688 & 18 & 8 & 32 \\
\hline & Nov & 2647 & 6335 & 26 & 9 & 41 \\
\hline & Dez & 1195 & 7530 & 20 & 2 & 43 \\
\hline \multirow[t]{12}{*}{94} & Jan & 758 & 8288 & 29 & 5 & 48 \\
\hline & Fev & 1310 & 9598 & 22 & 2 & 50 \\
\hline & Mar & 1112 & 10710 & 21 & 2 & 52 \\
\hline & Abr & 447 & 11157 & 24 & 4 & 56 \\
\hline & Maio & 1528 & 12685 & 28 & 5 & 61 \\
\hline & Jun & 78 & 12763 & 13 & 1 & 62 \\
\hline & Jul & 45 & 12808 & 10 & 0 & 62 \\
\hline & Ago & 158 & 12966 & 12 & 2 & 64 \\
\hline & Set & 138 & 13104 & 14 & 0 & 64 \\
\hline & Out & 1599 & 14703 & 27 & 6 & 70 \\
\hline & Nov & 1892 & 16595 & 23 & 4 & 74 \\
\hline & Dez & 1795 & 18390 & 23 & 5 & 79 \\
\hline \multirow[t]{4}{*}{95} & Jan & 2274 & 20664 & 21 & 1 & 80 \\
\hline & Fev & 2443 & 23107 & 24 & 1 & 81 \\
\hline & Mar & 2556 & 25663 & 46 & 4 & 85 \\
\hline & $\mathrm{Abr}$ & 927 & 26590 & 30 & 1 & 86 \\
\hline
\end{tabular}


Tab.22: Número de individuos $(\mathrm{N})$ e de espécies $(\mathrm{S})$ por coleta, número adicionado de espécies (Sadic.) a cada coleta e número acumulado de indivíduos (Nacum.) e espécies (Sacum.) obtidos em cada ano. Coletas em armadilha tipo Shannon em ambiente de mata residual ("mata A" - Fazenda Experimental do IAC Pariquera-Açu, SP).

\begin{tabular}{|c|c|c|c|c|c|c|}
\hline Ano & Coleta & $N$ & Nacum. & $\mathrm{S}$ & Sadic. & Sacum. \\
\hline \multirow[t]{12}{*}{92} & $\operatorname{Jan}$ & 448 & 448 & 32 & 32 & 32 \\
\hline & Fev & 961 & 1409 & 35 & 7 & 39 \\
\hline & Mar & 5266 & 6675 & 48 & 20 & 59 \\
\hline & $A b r$ & 525 & 7200 & 34 & 2 & 61 \\
\hline & Maio & 994 & 8194 & 18 & 2 & 63 \\
\hline & Jun & 209 & 8403 & 16 & 0 & 63 \\
\hline & Jul & 171 & 8574 & 18 & 3 & 66 \\
\hline & Ago & 100 & 8674 & 17 & 4 & 70 \\
\hline & Set & 104 & 8778 & 14 & 3 & 73 \\
\hline & Out & 100 & 8878 & 16 & 2 & 75 \\
\hline & Nov & 726 & 9604 & 22 & 2 & 77 \\
\hline & Dez & 2419 & 12023 & 37 & 2 & 79 \\
\hline \multirow[t]{12}{*}{93} & Jan & 162 & 162 & 23 & 23 & 23 \\
\hline & Fev & 2948 & 3110 & 64 & 48 & 71 \\
\hline & Mar & 3092 & 6202 & 62 & 19 & 90 \\
\hline & $\mathrm{Abr}$ & 2197 & 8399 & 41 & 3 & 93 \\
\hline & Maio & 475 & 8874 & 29 & 0 & 93 \\
\hline & Jun & 83 & 8957 & 18 & 0 & 93 \\
\hline & Jul & 353 & 9310 & 32 & 6 & 99 \\
\hline & Ago & 203 & 9513 & 27 & 2 & 101 \\
\hline & Set & 170 & 9683 & 21 & 2 & 103 \\
\hline & Out & 769 & 10452 & 33 & 2 & 105 \\
\hline & Nov & 827 & 11279 & 17 & 1 & 106 \\
\hline & Dez & 538 & 11817 & 23 & 0 & 106 \\
\hline \multirow[t]{12}{*}{94} & Jan & 697 & 697 & 28 & 28 & 28 \\
\hline & Fev & 783 & 1480 & 38 & 16 & 44 \\
\hline & Mar & 1047 & 2527 & 36 & 11 & 55 \\
\hline & $A b r$ & 960 & 3487 & 41 & 12 & 67 \\
\hline & Maio & 152 & 3639 & 18 & 0 & 67 \\
\hline & Jun & 177 & 3816 & 31 & 4 & 71 \\
\hline & Jul & 100 & 3916 & 15 & 0 & 71 \\
\hline & Ago & 383 & 4299 & 27 & 2 & 73 \\
\hline & Set & 70 & 4369 & 18 & 0 & 73 \\
\hline & Out & 240 & 4609 & 30 & 1 & 74 \\
\hline & Nov & 232 & 4841 & 26 & 1 & 75 \\
\hline & Dez & 234 & 5075 & 26 & 2 & 77 \\
\hline
\end{tabular}


Tab.23: Número de indivíduos (N) e de espécies (S) por coleta, número adicionado de espécies (Sadic.) a cada coleta $\mathrm{e}$ número acumulado de indivíduos (Nacum.) e espécies (Sacum.) obtidos no período de 3 anos. Coletas em armadilha tipo Shannon em ambiente de mata residual ("mata A" Fazenda Experimental do IAC - Pariquera-Açu, SP).

\begin{tabular}{|c|c|c|c|c|c|c|}
\hline Ano & Coleta & $\mathrm{N}$ & Nacum. & $S$ & Sadic. & Sacum. \\
\hline \multirow[t]{12}{*}{92} & Jan & 448 & 448 & 32 & 32 & 32 \\
\hline & Fev & 961 & 1409 & 35 & 7 & 39 \\
\hline & Mar & 5266 & 6675 & 48 & 20 & 59 \\
\hline & $A b r$ & 525 & 7200 & 34 & 2 & 61 \\
\hline & Maio & 994 & 8194 & 18 & 2 & 63 \\
\hline & Jun & 209 & 8403 & 16 & 0 & 63 \\
\hline & Jul & 171 & 8574 & 18 & 3 & 66 \\
\hline & Ago & 100 & 8674 & 17 & 4 & 70 \\
\hline & Set & 104 & 8778 & 14 & 3 & 73 \\
\hline & Out & 100 & 8878 & 16 & 2 & 75 \\
\hline & Nov & 726 & 9604 & 22 & 2 & 77 \\
\hline & Dez & 2419 & 12023 & 37 & 2 & 79 \\
\hline \multirow[t]{12}{*}{93} & Jan & 162 & 12185 & 23 & 3 & 82 \\
\hline & Fev & 2948 & 15133 & 64 & 12 & 94 \\
\hline & Mar & 3092 & 18225 & 62 & 11 & 105 \\
\hline & $A b r$ & 2197 & 20422 & 41 & 3 & 108 \\
\hline & Maio & 475 & 20897 & 29 & 0 & 108 \\
\hline & Jun & 83 & 20980 & 18 & 0 & 108 \\
\hline & Jul & 353 & 21333 & 32 & 4 & 112 \\
\hline & Ago & 203 & 21536 & 27 & 2 & 114 \\
\hline & Set & 170 & 21706 & 21 & 0 & 114 \\
\hline & Out & 769 & 22475 & 33 & 1 & 115 \\
\hline & Nov & 827 & 23302 & 17 & 1 & 116 \\
\hline & Dez & 538 & 23840 & 23 & 0 & 116 \\
\hline \multirow[t]{12}{*}{94} & Jan & 697 & 24537 & 28 & 2 & 118 \\
\hline & Fev & 783 & 25320 & 38 & 0 & 118 \\
\hline & Mar & 1047 & 26367 & 36 & 1 & 119 \\
\hline & $A b r$ & 960 & 27327 & 41 & 0 & 119 \\
\hline & Maio & 152 & 27479 & 18 & 0 & 119 \\
\hline & Jun & 177 & 27656 & 31 & 0 & 119 \\
\hline & Jul & 100 & 27756 & 15 & 0 & 119 \\
\hline & Ago & 383 & 28139 & 27 & 1 & 120 \\
\hline & Set & 70 & 28209 & 18 & 0 & 120 \\
\hline & Out & 240 & 28449 & 30 & 0 & 120 \\
\hline & Nov & 232 & 28681 & 26 & 0 & 120 \\
\hline & Dez & 234 & 28915 & 26 & 0 & 120 \\
\hline
\end{tabular}


Tab.24: Número de indivíduos (N) e de espécies (S) por coleta, número adicionado de espécies (Sadic.) a cada coleta e número acumulado de indivíduos (Nacum.) e espécies (Sacum.) obtidos em cada ano. Coletas em armadilha tipo Shannon em ambiente de mata residual ("mata B" - Fazenda Experimental do IAC Pariquera-Aç, SP).

\begin{tabular}{|c|c|c|c|c|c|c|}
\hline Ano & Coleta & $\mathrm{N}$ & Nacum. & $S$ & Sadic. & Sacum \\
\hline \multirow[t]{11}{*}{92} & Fev & 135 & 135 & 25 & 25 & 25 \\
\hline & Mar & 538 & 673 & 23 & 8 & 33 \\
\hline & Abr & 342 & 1015 & 37 & 15 & 48 \\
\hline & Maio & 387 & 1402 & 20 & 1 & 49 \\
\hline & Jun & 272 & 1674 & 18 & 2 & 51 \\
\hline & Jul & 32 & 1706 & 4 & 0 & 51 \\
\hline & Ago & 77 & 1783 & 13 & 3 & 54 \\
\hline & Set & 360 & 2143 & 22 & 3 & 57 \\
\hline & Out & 726 & 2869 & 32 & 8 & 65 \\
\hline & Nov & 693 & 3562 & 24 & 3 & 68 \\
\hline & Dez & 581 & 4143 & 21 & 1 & 69 \\
\hline \multirow[t]{12}{*}{93} & Jan & 544 & 544 & 30 & 30 & 30 \\
\hline & Fev & 1729 & 2273 & 43 & 17 & 47 \\
\hline & Mar & 2325 & 4598 & 51 & 18 & 65 \\
\hline & $A b r$ & 1034 & 5632 & 37 & 1 & 66 \\
\hline & Maio & 390 & 6022 & 22 & 2 & 68 \\
\hline & Jun & 269 & 6291 & 13 & 1 & 69 \\
\hline & Jul & 208 & 6499 & 14 & 0 & 69 \\
\hline & Ago & 630 & 7129 & 24 & 5 & 74 \\
\hline & Set & 384 & 7513 & 18 & 0 & 74 \\
\hline & Out & 921 & 8434 & 30 & 1 & 75 \\
\hline & Nov & 1013 & 9447 & 24 & 2 & 77 \\
\hline & Dez & 760 & 10207 & 20 & 2 & 79 \\
\hline \multirow[t]{12}{*}{94} & Jan & 693 & 693 & 24 & 24 & 24 \\
\hline & Fev & 1267 & 1960 & 33 & 14 & 38 \\
\hline & Mar & 1005 & 2965 & 44 & 18 & 56 \\
\hline & $A b r$ & 349 & 3314 & 31 & 8 & 64 \\
\hline & Maio & 245 & 3559 & 24 & 4 & 68 \\
\hline & Jun & 48 & 3607 & 12 & 1 & 69 \\
\hline & Jul & 108 & 3715 & 13 & 0 & 69 \\
\hline & Ago & 189 & 3904 & 22 & 1 & 70 \\
\hline & Set & 110 & 4014 & 18 & 3 & 73 \\
\hline & Out & 44 & 4058 & 11 & 1 & 74 \\
\hline & Nov & 236 & 4294 & 31 & 2 & 76 \\
\hline & Dez & 389 & 4683 & 26 & 5 & 81 \\
\hline
\end{tabular}


Tab.25: Número de individuos ( $\mathrm{N}$ ) e de espécies (S) por coleta, número adicionado de espécies (Sadic.) a cada coleta e número acumulado de individuos (Nacum.) e espécies (Sacum.) obtidos no periodo de 3 anos. Coletas em armadilha tipo Shannon em ambiente de mata residual ("mata B" - Fazenda Experimental do IAC - Pariquera-Açu, SP).

\begin{tabular}{|c|c|c|c|c|c|c|}
\hline Ano & Coleta & $N$ & Nacum. & s & Sadic. & Sacum \\
\hline \multirow[t]{11}{*}{92} & Fev & 135 & 135 & 25 & 25 & 25 \\
\hline & Mar & 538 & 673 & 23 & 8 & 33 \\
\hline & $A b r$ & 342 & 1015 & 37 & 15 & 48 \\
\hline & Maio & 387 & 1402 & 20 & 1 & 49 \\
\hline & Jun & 272 & 1674 & 18 & 2 & 51 \\
\hline & Jul & 32 & 1706 & 4 & 0 & 51 \\
\hline & Ago & 77 & 1783 & 13 & 3 & 54 \\
\hline & Set & 360 & 2143 & 22 & 3 & 57 \\
\hline & Out & 726 & 2869 & 32 & 8 & 65 \\
\hline & Nov & 693 & 3562 & 24 & 3 & 68 \\
\hline & Dez & 581 & 4143 & 21 & 1 & 69 \\
\hline \multirow[t]{12}{*}{93} & $\operatorname{Jan}$ & 544 & 4687 & 30 & $\overline{0}$ & 69 \\
\hline & Fev & 1729 & 6416 & 43 & 6 & 75 \\
\hline & Mar & 2325 & 8741 & 51 & 11 & 86 \\
\hline & $\mathrm{Abr}$ & 1034 & 9775 & 37 & 1 & 87 \\
\hline & Maio & 390 & 10165 & 22 & 1 & 88 \\
\hline & Jun & 269 & 10434 & 13 & 1 & 89 \\
\hline & Jul & 208 & 10642 & 14 & 0 & 89 \\
\hline & Ago & 630 & 11272 & 24 & 4 & 93 \\
\hline & Set & 384 & 11656 & 18 & 0 & 93 \\
\hline & Out & 921 & 12577 & 30 & 0 & 93 \\
\hline & Nov & 1013 & 13590 & 24 & 1 & 94 \\
\hline & Dez & 760 & 14350 & 20 & 1 & 95 \\
\hline \multirow[t]{12}{*}{94} & Jan & 693 & 15043 & 24 & 2 & 97 \\
\hline & Fev & 1267 & 16310 & 33 & 1 & 98 \\
\hline & Mar & 1005 & 17315 & 44 & 2 & 100 \\
\hline & Abr & 349 & 17664 & 31 & 5 & 105 \\
\hline & Maio & 245 & 17909 & 24 & 2 & 107 \\
\hline & Jun & 48 & 17957 & 12 & 0 & 107 \\
\hline & Jul & 108 & 18065 & 13 & 0 & 107 \\
\hline & Ago & 189 & 18254 & 22 & 0 & 107 \\
\hline & Set & 110 & 18364 & 18 & 0 & 107 \\
\hline & Out & 44 & 18408 & 11 & 1 & 108 \\
\hline & Nov & 236 & 18644 & 31 & 1 & 109 \\
\hline & Dez & 389 & 19033 & 26 & 4 & 113 \\
\hline
\end{tabular}


Tab.26: Número de individuos $(\mathrm{N})$ e de espécies $(\mathrm{S})$ por coleta, número adicionado de espécies (Sadic.) a cada coleta e número acumulado de individuos (Nacum.) e espécies (Sacum.) obtidos em cada ano. Coletas em armadilha tipo Shannon em área de cultivo irrigado de arroz (Fazenda Experimental do IAC Pariquera-Açu, SP)

\begin{tabular}{ccccccc}
\hline Ano & Coleta & N & Nacum. & S & Sadic. & Sacum. \\
\hline 92 & Jan & 706 & 706 & 31 & 31 & 31 \\
& Fev & 1384 & 2090 & 37 & 10 & 41 \\
& Mar & 1337 & 3427 & 32 & 1 & 42 \\
& Abr & 1924 & 5351 & 38 & 6 & 48 \\
& Maio & 2696 & 8047 & 41 & 5 & 53 \\
& Jun & 4236 & 12283 & 31 & 1 & 54 \\
& Jul & 498 & 12781 & 18 & 0 & 54 \\
& Ago & 479 & 13260 & 32 & 2 & 56 \\
& Set & 561 & 13821 & 24 & 1 & 57 \\
& Out & 1081 & 14902 & 36 & 3 & 60 \\
& Nov & 1748 & 16650 & 28 & 1 & 61 \\
& Dez & 1874 & 18524 & 32 & 0 & 61 \\
\hline 93 & Jan & 3282 & 3282 & 31 & 31 & 31 \\
& Fev & 862 & 4144 & 26 & 10 & 41 \\
& Mar & 22243 & 26387 & 43 & 14 & 55 \\
& Abr & 5753 & 32140 & 49 & 8 & 63 \\
Maio & 1329 & 33469 & 33 & 1 & 64 \\
& Jun & 634 & 34103 & 25 & 0 & 64 \\
Jul & 618 & 34721 & 28 & 0 & 64 \\
& Ago & 191 & 34912 & 14 & 1 & 65 \\
Set & 276 & 35188 & 19 & 0 & 65 \\
Out & 2185 & 37373 & 28 & 2 & 67 \\
Nov & 7377 & 44750 & 35 & 1 & 68 \\
Dez & 3842 & 48592 & 26 & 0 & 68 \\
\hline & & & & &
\end{tabular}


xxxvii

Tab.27: Número de individuos (N) e de espécies (S) por coleta, número adicionado de espécies (Sadic.) a cada coleta $\mathrm{e}$ número acumulado de indivíduos (Nacum.) e espécies (Sacum.) obtidos em 2 anos. Coletas em armadilha tipo Shannon em área de cultivo irrigado de arroz (Fazenda Experimental do IAC - Pariquera-Açu, SP).

\begin{tabular}{ccccccc}
\hline Ano & Coleta & $N$ & Nacum. & S & Sadic. & Sacum. \\
\hline 92 & Jan & 706 & 706 & 31 & 31 & 31 \\
& Fev & 1384 & 2090 & 37 & 10 & 41 \\
& Mar & 1337 & 3427 & 32 & 1 & 42 \\
& Abr & 1924 & 5351 & 38 & 6 & 48 \\
& Maio & 2696 & 8047 & 41 & 5 & 53 \\
& Jun & 4236 & 12283 & 31 & 1 & 54 \\
& Jul & 498 & 12781 & 18 & 0 & 54 \\
& Ago & 479 & 13260 & 32 & 2 & 56 \\
& Set & 561 & 13821 & 24 & 1 & 57 \\
& Out & 1081 & 14902 & 36 & 3 & 60 \\
& Nov & 1748 & 16650 & 28 & 1 & 61 \\
& Dez & 1874 & 18524 & 32 & 0 & 61 \\
\hline 93 & Jan & 3282 & 21806 & 31 & 4 & 65 \\
& Fev & 862 & 22668 & 26 & 1 & 66 \\
& Mar & 22243 & 44911 & 43 & 3 & 69 \\
& Abr & 5753 & 50664 & 49 & 2 & 71 \\
& Maio & 1329 & 51993 & 33 & 0 & 71 \\
& Jun & 634 & 52627 & 25 & 0 & 71 \\
& Jut & 618 & 53245 & 28 & 0 & 71 \\
& Ago & 191 & 53436 & 14 & 0 & 71 \\
Set & 276 & 53712 & 19 & 0 & 71 \\
& Out & 2185 & 55897 & 28 & 1 & 72 \\
Nov & 7377 & 63274 & 35 & 0 & 72 \\
Dez & 3842 & 67116 & 26 & 0 & 72 \\
\hline
\end{tabular}


Tab.28: Espécies constantes em pelo menos um dos ambientes de estudo - mata primária (FT, SG - Cananéia, SP), mata residual (MA, MB Pariquera-Açu, SP) e área de cultivo irrigado de arroz (AR - Pariquera-Açu, SP), considerados os respectivos períodos de coletas em armadilha tipo Shannon (FT - 12, SG - 23, MA e MB - 36, AR - 24 meses)

\begin{tabular}{|c|c|c|c|c|c|}
\hline Espécie & FT & SG & MA & MB & AR \\
\hline Ad.squamipennis & & & $z$ & & $z$ \\
\hline Ae.albifasciatus & & & & & $z$ \\
\hline Ae.albopictus & & & $z$ & $z$ & \\
\hline Ae.argyrothorax & y & $z$ & & & \\
\hline Ae.crinifer & & $z$ & $z$ & $z$ & $y$ \\
\hline Ae.fluviatilis & & & $z$ & & \\
\hline Ae.fulus & $y$ & & $y$ & y & $y$ \\
\hline Ae.gr.Serratus(hastatus/oligopistus) & $y$ & $y$ & $z$ & $z$ & \\
\hline Ae.gr.Serratus(serratus/nubilus) & $x$ & $x$ & $x$ & $x$ & $\mathrm{x}$ \\
\hline Ae.hastatus & $z$ & $z$ & & & \\
\hline Ae.nubilus & & & $z$ & $z$ & $z$ \\
\hline Ae.oligopistus & $y$ & $z$ & & $z$ & \\
\hline Ae.prox.perventor ou hortator & $x$ & z & & $z$ & \\
\hline Ae.scapulanis & $x$ & $x$ & $x$ & $x$ & $x$ \\
\hline Ae.serratus & $x$ & y & $x$ & $x$ & $x$ \\
\hline An.albitarsis esp. A & & & $z$ & & $x$ \\
\hline An.albitarsis esp. B & & $z$ & $z$ & & $x$ \\
\hline An.(Ano.)eiseni & $z$ & & & & \\
\hline An.(Nys.) secao Argyritarsis ou Albimanus & & & $z$ & & $y$ \\
\hline An.(Ste.)kompi & $z$ & & & & \\
\hline An.(Ste.)kompicanorii & $z$ & $z$ & & & \\
\hline An.(Ste.)nimbusftomasi & $y$ & & & & \\
\hline An.albitarsis & & & $z$ & & $x$ \\
\hline An.bellator & $x$ & $x$ & & $z$ & \\
\hline An.cruzii & $x$ & $x$ & y & $x$ & $y$ \\
\hline An.evansae & $z$ & & $z$ & $z$ & $x$ \\
\hline An.evansae/galvaoi & & & & & $z$ \\
\hline Anffuminensis & & & & $z$ & \\
\hline An.galvaoi & $z$ & & $z$ & & y \\
\hline An.grupo punctimacula & $z$ & & $z$ & $z$ & $y$ \\
\hline An.intermedius & $x$ & $z$ & $y$ & $z$ & $x$ \\
\hline An.htzii & $z$ & & & & \\
\hline An.oswaldoi & $z$ & & $z$ & $z$ & $x$ \\
\hline An.prox.mediopunctatus & $x$ & $z$ & $x$ & $x$ & $z$ \\
\hline An.strodei & $z$ & & $z$ & & $x$ \\
\hline An.triannulatus & & & $z$ & $z$ & y \\
\hline Cq.albicosta & & & $z$ & $z$ & $z$ \\
\hline Cq.albifera & & & & & $z$ \\
\hline Cq.chrysonotum & $z$ & $x$ & $y$ & $z$ & $y$ \\
\hline Cq.chrysonotum ou albifera & $x$ & $x$ & $x$ & $x$ & $x$ \\
\hline Cq.hermanoi & & $z$ & $x$ & $z$ & $x$ \\
\hline
\end{tabular}

$\mathrm{x}=$ espécies constantes (presentes em mais de $50 \%$ das coletas) $y=e s p e c i e s$ acessórias (presentes em 25-50\% das coletas)

$z=e s p e c i e s$ acidentais (presentes em menos de $25 \%$ das coletas) 
(cont.)

\begin{tabular}{|c|c|c|c|c|c|}
\hline Espécie & FT & SG & MA & MB & AR \\
\hline Cq.juxtamansonia & $z$ & $y$ & $x$ & $z$ & $x$ \\
\hline Cq.nigricans & & $z$ & $z$ & $z$ & $y$ \\
\hline Cq.venezuelensis & $y$ & $x$ & $x$ & $x$ & $x$ \\
\hline Cx.(Aed.)amazonensis & & & $y$ & $z$ & $z$ \\
\hline Cx.(Cux.) grupo Coronator & $x$ & $x$ & $x$ & $y$ & $x$ \\
\hline Cx.(Cux.)bidens & & $z$ & $z$ & $z$ & \\
\hline Cx. (Cux.)chidesteri & & $z$ & $z$ & $z$ & $z$ \\
\hline Cx. (Cux, )comiger & & & $z$ & & $z$ \\
\hline Cx. (Cux.)coronator & & & $z$ & & \\
\hline Cx.(Cux.)declarator & $z$ & & $z$ & $z$ & \\
\hline Cx.(Cux.)dolosus & & $z$ & $z$ & $z$ & \\
\hline Cx. $(C u x$.$) dolosus/eduardoi$ & & & $z$ & $z$ & \\
\hline Cx.(Cux.)hygrus & $z$ & & $z$ & $z$ & \\
\hline Cx.(Cux.)mollis & $y$ & $z$ & $z$ & $z$ & \\
\hline Cx.(Cux.)nignipalpus & $x$ & $y$ & $x$ & $x$ & $x$ \\
\hline Cx.(Cux.)sp. & $x$ & $x$ & $x$ & $x$ & $x$ \\
\hline Cx. (Cux.)usquatus & $z$ & & & & \\
\hline Cx.(Lut.)bigoti & & $z$ & $z$ & $z$ & $z$ \\
\hline Cx.(Mcx.) gr. pleuristriatus & & & & $z$ & \\
\hline Cx. (Mcx.) lanei & $z$ & $z$ & & & \\
\hline Cx. (Mcx.)dubitans & $z$ & & & & \\
\hline CX.(Mcx.)grupo imitator & & $z$ & $z$ & $z$ & $z$ \\
\hline Cx.(Mcx.)imitator & $y$ & $z$ & $z$ & $z$ & \\
\hline Cx.(Mcx.)neglectus & $z$ & z & & $z$ & \\
\hline Cx.(Mcx.)pleuristriatus & $z$ & $z$ & $z$ & & \\
\hline Cx.(Mel.)abonnenci & & $z$ & & & \\
\hline Cx.(Mel.)aliciae & $y$ & & & $z$ & \\
\hline Cx.(Mel.)aureonotatus & $z$ & & $z$ & $z$ & $y$ \\
\hline Cx.(Mel.)bastaganius & $z$ & $z$ & $y$ & $y$ & $y$ \\
\hline Cx.(Mel.)caudelli & & & & $z$ & \\
\hline Cx.(Mel.)corentynensis & $y$ & $z$ & & & \\
\hline Cx.(Mel.)delpontei & & & $z$ & $z$ & $z$ \\
\hline Cx.(Mel.)distinguendus & $z$ & & $z$ & $z$ & \\
\hline Cx.(Mel.)dunni & & $z$ & $y$ & $z$ & \\
\hline Cx.(Mel.)eastor & & & $z$ & $z$ & \\
\hline Cx.(Mel.)ensiformis & & & $z$ & & \\
\hline Cx.(Mel.)evansae & $z$ & $z$ & $z$ & $z$ & \\
\hline Cx.(Mel.)faurani & & & $z$ & $z$ & \\
\hline Cx.(Mel.)galvaoi & $y$ & $y$ & & z & \\
\hline Cx.(Mel.)grupo Atratus & $x$ & $z$ & $y$ & $x$ & $z$ \\
\hline Cx.(Mel.)grupo Intrincatus & $z$ & $z$ & $z$ & $z$ & \\
\hline CX.(Mel.)grupo Pilosus & $y$ & $y$ & $y$ & $y$ & $y$ \\
\hline Cx.(Mel.)inadmirabilis & & & $z$ & & $z$ \\
\hline Cx.(Mel)innovator & & & $z$ & $z$ & \\
\hline Cx.(Mel.)intrincatus & & $z$ & $z$ & & \\
\hline Cx.(Mel.)lopesi & & & $z$ & & \\
\hline
\end{tabular}

$x=$ especies constantes (presentes em mais de $50 \%$ das coletas) $y=$ especies acessorias (presentes em $25 \sim 50 \%$ das coletas)

$z=e s p e c i e s$ acidentais (presentes em menos de $25 \%$ das coletas) 
(cont.)

\begin{tabular}{|c|c|c|c|c|c|}
\hline Espécie & FT & $S G$ & MA & $\mathrm{MB}$ & AR \\
\hline Cx.(Mel.)misionensis & $\mathbf{z}$ & $\mathbf{z}$ & $z$ & $z$ & \\
\hline Cx.(Mel.)ocellatus & $y$ & $z$ & & $z$ & \\
\hline Cx,(Mel.)ocossa & & $z$ & $y$ & $z$ & $x$ \\
\hline Cx.(Mel.)oodipus & $y$ & $z$ & $y$ & $z$ & $z$ \\
\hline Cx.(Mel.)orfilai & & & & $z$ & \\
\hline Cx.(Mel.)pedroi & $z$ & $z$ & $y$ & $z$ & \\
\hline Cx.(Mel.)pereyrai & & & $z$ & $z$ & \\
\hline Cx.(Mel.)pilosus & $z$ & & $z$ & $z$ & \\
\hline Cx.(Mel.)plectoporpe & $z$ & $z$ & $y$ & $z$ & $y$ \\
\hline Cx.(Mel.)rabelloi & & $z$ & $z$ & $z$ & \\
\hline Cx.(Mel.)nibeirensis & $x$ & $x$ & $x$ & $x$ & $x$ \\
\hline Cx.(Mel.)sacchettae & $x$ & $x$ & $x$ & $x$ & $x$ \\
\hline$C_{x}(\mathrm{Mel}) s p$. & & $z$ & $z$ & $z$ & $z$ \\
\hline Cx.(Mel).)sp sec Melanoconion & & & $z$ & & \\
\hline Cx.(Mel)spissipes & & & $z$ & $y$ & \\
\hline Cx.(Mel.)taeniopus & $y$ & $z$ & $z$ & $y$ & \\
\hline Cx.(Mel.)theobaidi V. R. & $y$ & $z$ & $y$ & $z$ & $y$ \\
\hline Cx.(Mel.)zeteki & $y$ & $z$ & $y$ & $z$ & $z$ \\
\hline Li.dumami & $y$ & $x$ & $x$ & $x$ & $z$ \\
\hline Liffavisetosus & $\mathbf{z}$ & $y$ & $x$ & $x$ & \\
\hline Ma.(Man.)cerqueirai & & & & & $z$ \\
\hline Ma.(Man.)ffaveola & & & $z$ & $z$ & $z$ \\
\hline Ma.(Man.)humeralis & & & $z$ & & $x$ \\
\hline Ma.(Man.)indubitans & & $z$ & $x$ & $x$ & $x$ \\
\hline Ma.(Man.)pseudotitillans & & $z$ & $z$ & & $y$ \\
\hline Ma.(Man.)sp & $z$ & $y$ & $x$ & $y$ & $\mathbf{z}$ \\
\hline Ma.(Man.)titillans & & $z$ & $z$ & $z$ & $y$ \\
\hline Ph.pallidiventer & $z$ & & & & \\
\hline Ph.prox.flabellata & $z$ & & $z$ & $z$ & \\
\hline Ph.prox.longirostris & $z$ & & & & \\
\hline Ph.prox. palmata/diabolica & $y$ & $z$ & $z$ & $y$ & \\
\hline Ph.prox.theobaldi & $z$ & $z$ & $z$ & $z$ & \\
\hline Ph.davisi & $z$ & $z$ & $z$ & $z$ & \\
\hline Ph.fiabellata & $x$ & $z$ & & & \\
\hline Ph.longirostris & $z$ & & & & \\
\hline Ph.palmata/diabolica & $x$ & $y$ & $\mathbf{z}$ & $z$ & \\
\hline Ph.quasilongirostris & $\mathrm{y}$ & $y$ & $y$ & $y$ & \\
\hline Ph.theobaldi & $y$ & $y$ & $z$ & $z$ & \\
\hline Phoniomyia sp & & $z$ & & $z$ & \\
\hline Ps.albigenu & $z$ & $z$ & $y$ & $y$ & $y$ \\
\hline Ps.albigenutvaripes & & & $y$ & $z$ & $y$ \\
\hline Ps.albipes & & $z$ & $z$ & $z$ & \\
\hline Ps.ciliata & & & & & $x$ \\
\hline Ps.cingulata & & & $z$ & $z$ & $y$ \\
\hline Ps.confinnis & & & & & $y$ \\
\hline Ps.discrucians & & & $z$ & $z$ & $x$ \\
\hline
\end{tabular}

$x=e$ pécies constantes (presentes em mais de $50 \%$ das coletas)

$y=e$ pécies acessórias (presentes em 25 50\% das coletas)

$z=e s p e c i e s$ acidentais (presentes em menos de $25 \%$ das coletas) 
(cont.)

\begin{tabular}{|c|c|c|c|c|c|}
\hline Espécie & FT & SG & $\mathrm{MA}$ & MB & AR \\
\hline Ps.ferox & $x$ & $y$ & $x$ & $x$ & $z$ \\
\hline Ps.tutzii & $y$ & & $z$ & $y$ & \\
\hline Ru.humboldt & $y$ & $z$ & $z$ & $z$ & \\
\hline Ru.prox.reversa & $y$ & $z$ & z & $z$ & \\
\hline Ru.reversa & $x$ & $y$ & $y$ & $y$ & \\
\hline Ru.theobaldi & $z$ & $z$ & $z$ & $z$ & \\
\hline Sa.chloroptenus & & & $z$ & $z$ & \\
\hline Sh.fluviatilis & $z$ & & & & \\
\hline Tr. pallid/castroi/similis & $z$ & & & & \\
\hline Tr.digitatum & $z$ & & $z$ & $y$ & \\
\hline Ur.apicalis & & & $z$ & & $z$ \\
\hline Ur.calosomata & $z$ & $y$ & $y$ & $z$ & \\
\hline Ur.geometrica & $z$ & $x$ & $z$ & $z$ & $y$ \\
\hline Ur.lowii & & $z$ & $z$ & $z$ & $z$ \\
\hline Ur.mathesoni & $z$ & & $\mathbf{z}$ & $z$ & \\
\hline Ur.nataliae & & & $z$ & $z$ & \\
\hline Ur.pulchemima & & $z$ & $z$ & & $z$ \\
\hline Wy pertinans & & & & $\mathbf{z}$ & $z$ \\
\hline Wy.felicialpampeites & $z$ & $y$ & $y$ & $y$ & \\
\hline Wy. airosaihowaAuteove & $y$ & & $z$ & $z$ & \\
\hline Wy.aporonoma & $z$ & & $y$ & $z$ & \\
\hline Wy.contusa & $x$ & $x$ & $x$ & $x$ & \\
\hline Wy.finlayi & $z$ & & & & \\
\hline Wy.mystes ou finlayi & $x$ & $z$ & $z$ & $z$ & \\
\hline Wy.shannoni & $y$ & $z$ & $z$ & $z$ & \\
\hline
\end{tabular}

$x=e s p e c i e s$ constantes (presentes em mais de $50 \%$ das coletas) y=especies acessórias (presentes em 25-50\% das coletas)

$z=e$ spécies acidentais (presentes em menos de $25 \%$ das coletas) 